Check for updates

Cite this: RSC Adv., 2019, 9, 23161

Received 11th June 2019

Accepted 12th July 2019

DOI: 10.1039/c9ra04389f

rsc.li/rsc-advances

\section{Recent synthetic advances in pyridine-based thermotropic mesogens}

\author{
Deepak Devadiga and T. N. Ahipa (D)* \\ Currently, numerous articles have reported pyridine-based thermotropic mesogens; however, reviews of \\ their synthetic methodologies are rare. Therefore, the present critical review describes the recent \\ synthetic advances in the field of pyridine-based thermotropic mesogens. Also, we discuss the various \\ types of thermotropic mesogens (such as calamitic, discotic, bent-shaped, polycatenar, and polymeric \\ mesogens) consisting of pyridine derivatives and their structure-property relationships.
}

\section{Introduction}

For certain organic compounds, samples do not move directly from the solid to the liquid state upon heating. Instead, they pass through an intermediate state called the liquid crystalline state. The compounds which exhibit this special feature are designated as liquid crystal materials. In general, liquid crystals are calamitic, discotic, polycatenar or bent-shaped organic molecules with terminal groups such as $-\mathrm{OR},-\mathrm{COOR},-\mathrm{Cl}$, and $-\mathrm{NO}_{2}$ and bridging groups such as $-\mathrm{C}=\mathrm{N}-,-\mathrm{COO}-,-\mathrm{NH}-(\mathrm{C}=$ $\mathrm{O})-$, and $-\mathrm{C}=\mathrm{C}-$. In general, liquid crystals are known for their unique characteristics. In fact, these molecules have high order and no or little translational freedom in their solid states. On the other hand, they do not have intrinsic order in the liquid state. The characteristic ordering in the liquid crystal state is between those in the solid and liquid states, i.e., the molecules in the liquid crystal state are almost parallel to each other and can flow like a liquid with translational freedom. Thus, liquid crystals have the fluidity of liquids and the layer ordering of solids.

It is interesting to note that the design and development of various heterocyclic-based thermotropic liquid crystals has gained the interest of researchers due to the stress-free modification of molecular shapes and their excellent ability to impart lateral and/or longitudinal dipoles. ${ }^{1-3}$ In fact, these features have helped further several photochemical and optical applications in areas of material science, such as organic photovoltaic devices, ${ }^{4-6}$ optical signal processing and storage, ${ }^{7}$ switching ferroelectric materials, ${ }^{8}$ and organic transistors. ${ }^{9}$ In the last few years, a pronounced number of thermotropic liquid crystalline materials with core moieties comprising heterocyclic rings have been designed, synthesized and characterized. These heterocyclic rings consist of electronegative hetero-atoms (such

Centre for Nano and Material Sciences, Jain University, Jain Global Campus, Jakkasandra Post, Ramanagara District, Bangalore-562 112, India. E-mail: tn. ahipa@jainuniversity.ac.in as nitrogen, oxygen, and sulphur), and the occupancy of these electronegative atoms frequently triggers diminished symmetry of the complete molecule and, thereby, develops powerful polar induction. ${ }^{7}$ Highly $\pi$-conjugated mesogens bearing heterocycles are showing enhanced use in organic photonics. ${ }^{10}$ In fact, fivemembered N-heterocyclic compounds are frequently fabricated in materials science due to their ability to further interact (or hydrogen bond) with metal atoms. ${ }^{7}$ Five-membered Nheterocyclic compounds are capable of building complexes with metals or may exhibit mesogenic natures through hydrogen bond donor-acceptor assemblies via specific interactions. Also, some of these compounds can be electron rich or deficient based on the ring nature and, therefore, can be utilized in organic electronic applications. In this context, pyrazoles have been efficiently implemented in the domain of metallo-mesogens; at the same time, triazoles have been utilized to produce supramolecular mesogens. ${ }^{7}$

The use of six-membered N-heterocyclic systems in mesogenic molecules moderately alters their molecular geometry in contrast with their benzene analogs. However, the site of the Nheteroatom has a significant impact on the dispersion forces, the dipole moment, the polarizability of the molecule and, inevitably, on the dielectric nature of the mesogen. ${ }^{11}$ Thus, depending on the heteroatom sites in mesomorphic derivatives, the dielectric anisotropy of mesogens can have negative or positive values. In the case of pyridazine derivatives, they have negative dielectric anisotropy because their dipole moment directions create an angle close to $90^{\circ}$ with a long molecular axis; in the case of pyrimidine derivatives, they have positive dielectric anisotropy because their dipole moments are directed along the molecular axis. ${ }^{3}$ However, the presence of a single nitrogen atom in the case of pyridine also alters the photophysical, electrical, and thermal mesogenic behaviours compared to carbocyclic derivatives. Inspired by these unique properties, we focus our present review article on recent advances in the field (such as the design, synthesis, and 
photophysical and liquid crystal properties) of pyridine-based compounds as our prime subject of interest.

\section{Pyridine-based molecules as thermotropic mesogens}

\subsection{Pyridine-based calamitic mesogens}

Calamitic mesogens are rod-like molecules wherein the length of the molecule is much greater than its width. The rigid core usually consists of aromatic rings (phenyl, biphenyl, etc.) and/or even heteroaromatic rings. In addition, certain alicyclic rings (cholesteryl, trans-4-cyclo hexyl, etc.) can be used. In general, these cores are connected either by covalent bonds or by linkage units (-COO-, $-\mathrm{N}=\mathrm{N}-,-\mathrm{CH}=\mathrm{N}-$ ). Moreover, these molecules contain terminal alkyl or alkoxy chains; in some cases, one of these chains is a polar group (CN, NCO, NCS, etc.). This section summarizes the recently developed synthetic methods for the preparation of different pyridine-based calamitic mesogens and their properties.

Liu et $a .^{12}$ synthesized two proton acceptor molecules bearing Schiff base linkages, i.e. $\mathrm{N}$-(pyridin-4-ylmethylene)-4(2,2,2-trifluoroethoxy)benzenamine (3) and 4-ethoxy- $N$ (pyridin-4-ylmethylene)benzenamine (5). These compounds were synthesized by reacting either 4-(2,2,2-trifluoroethoxy) aniline (1) or 4-ethoxyaniline (4) with pyridine 4-carbaldehyde (2) through condensation reactions. Further, they prepared a series of supramolecular hydrogen bonded liquid crystal complexes (7a-g, 8a-g) using these proton acceptor molecules $(3,5)$ and 4 -(n-alkoxy)benzoic acids $(\mathbf{6 a - g})$ in pyridine solvent (Scheme 1). The formation of hydrogen bonding between the pyridine derivative and 4-(n-alkoxy)benzoic acid was confirmed by comparison of the infrared spectra of the parent compounds and the hydrogen bonded complex. Polarizing optical microscopy (POM) and differential scanning calorimetry (DSC) studies on these hydrogen bonded complexes reveal that the fluorinated analogues have higher clearing temperatures than the non-fluorinated analogues; hence, the authors concluded that there is increased stability and a higher degree of order in the fluorine analogue-based hydrogen bonded complexes due to the presence of trifluoromethyl groups. Further, the fluorinated analogues exhibited smectic A phase and the non-fluorinated analogues showed nematic phase under POM examination; later, these mesophases were further confirmed by X-ray diffraction (XRD) studies. Moreover, the authors checked the stability of the imine bonds in the prepared compounds through hydrolysis reactions using water and ethanol in a $9: 1$ v/v ratio at room temperature. From this study, it was observed that the imine bonds are stable even after 7 days of continuous hydrolysis; the stability of the imine bonds is believed to be due to the existence of conjugation in the molecules.

Recently, He et al. ${ }^{13}$ synthesized a proton acceptor molecule, i.e. 4-(4-trans-propylcyclohexyl)phenol isonicotinate (11), with an ester linkage, and proton donor molecules, $(S)$-4-(2-<smiles>CC(=O)OCC(=O)c1ccc(N=Cc2ccncc2)cc1</smiles><smiles>CCOC(=O)c1ccc(N=Cc2ccncc2)cc1</smiles>

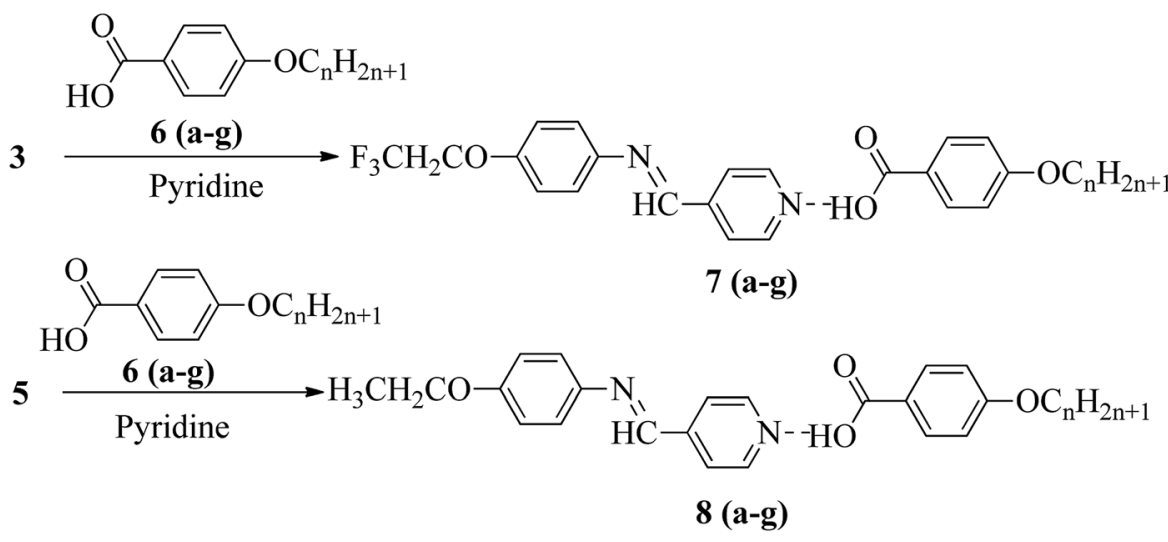

\begin{tabular}{|c|c|c|c|c|c|c|c|}
\hline $\mathbf{6 , 7 , 8}$ & $\mathbf{a}$ & $\mathbf{b}$ & $\mathbf{c}$ & $\mathbf{d}$ & $\mathbf{e}$ & $\mathbf{f}$ & $\mathbf{g}$ \\
\hline $\mathrm{n}$ & 4 & 5 & 6 & 7 & 8 & 10 & 12 \\
\hline
\end{tabular}


<smiles>O=C(O)c1ccncc1</smiles>

9<smiles></smiles>

10 alkyl-trans-

cyclohexylphenol,

11

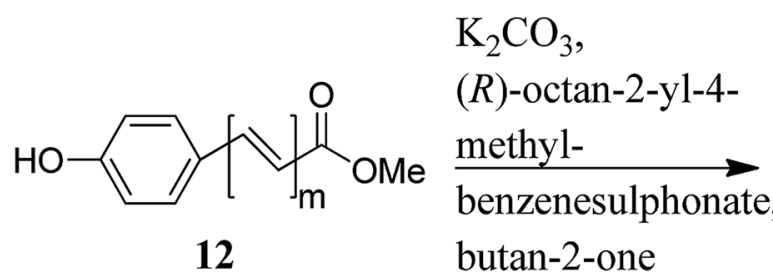

13

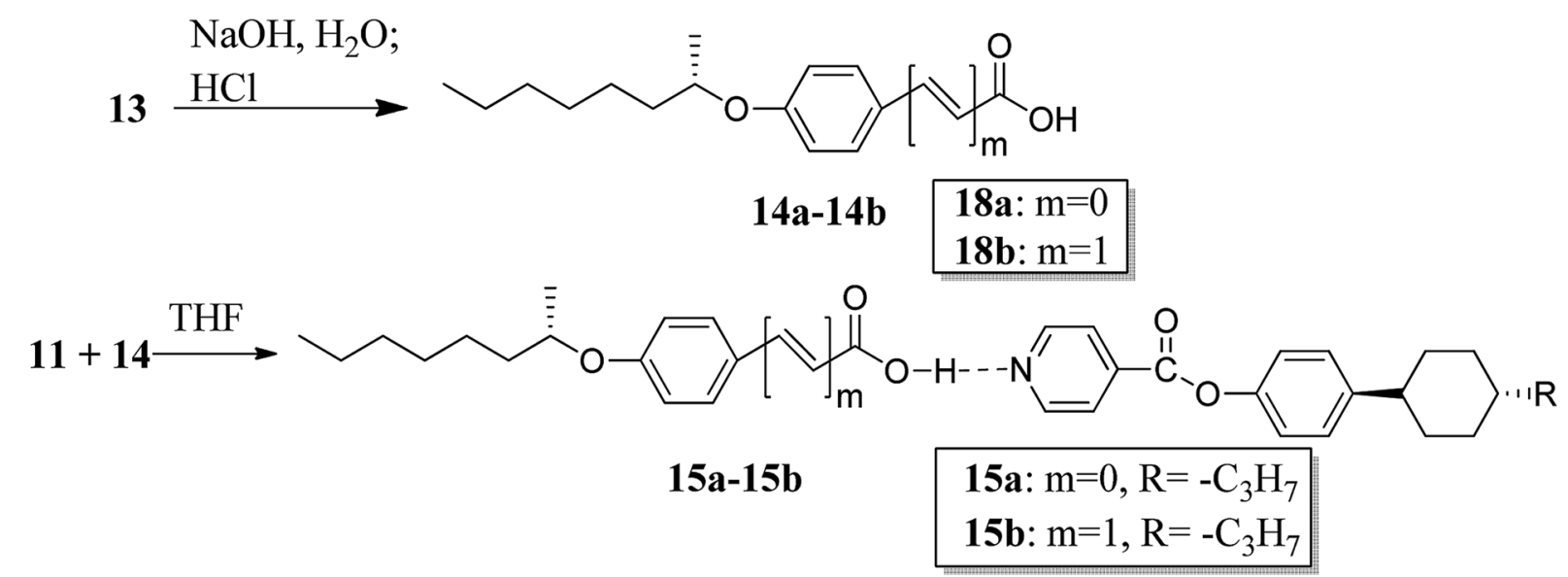

Scheme 2 Synthetic routes for the hydrogen bonded liquid crystals $15 \mathrm{a}$ and 15b.

octanyloxy)benzoic acid (14a) and (S)-4-(2-octanyloxy)cinnamic acid (14b), as shown in Scheme 2. These proton acceptor and donors are non-mesogenic in nature. Further, hydrogen bonded complexes were prepared by dissolving $1: 1,1: 2$, and $1: 3$ mole ratios of $\mathbf{1 1}$ and $\mathbf{1 4 a}$ or $14 \mathbf{b}$, respectively, in tetrahydrofuran (THF) and heating the resulting mixture to $60{ }^{\circ} \mathrm{C}$ for several minutes followed by solvent evaporation; this led to the formation of hydrogen bonded complexes. These hydrogen bonded complexes exhibited liquid crystalline properties. Mesophases such as smectic A (SmA), twisted-grain-

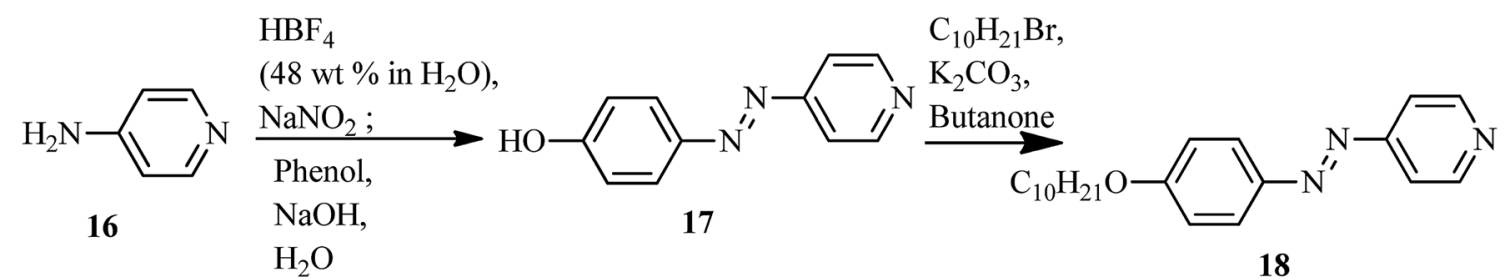

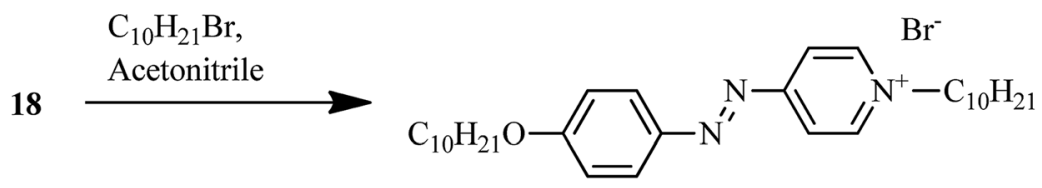

Scheme 3 Synthetic route of the ionic liquid crystal 19. 
boundary A (TGBA*) and chiral nematic phase $\left(\mathrm{N}^{*}\right)$ were observed in complex 15a, whereas complex 15b exhibited blue phase (BP*) in addition to the mesophases of complex 15a. Hydrogen bonded complex 15b in a mole ratio of $1: 2$ generated $\mathrm{BP}^{*}$ mesophase. Further, the $\mathrm{BP}^{*}$ phase range was extended to $10{ }^{\circ} \mathrm{C}$ during the cooling cycle. Density functional theory (DFT) studies showed that the flexible cinnamic-acid-derived liquid crystals have a bent shape; thus, they concluded that the flexible nature of the compounds plays a positive role in the stabilization of BPs and, thus, these complexes can be used in applications of photonic crystals and optoelectronics.

The first report on ionic liquid crystals with amphotropic behavior for the development of electrochemical biosensors was prepared by Zapp et al. ${ }^{\mathbf{1 4}}$ The synthetic scheme involves the diazotization of 4-aminopyridine (16) using tetrafluoroboric acid solution $\left(\mathrm{HBF}_{4}\right)$ (48 wt\% in water) and $\mathrm{NaNO}_{2}$. This yields the corresponding diazonium tetrafluoroborate salt. Further, the prepared diazonium salt is treated with phenol in a basic aqueous solution through an azo-coupling reaction to afford compound (17); alkylation of the phenolic hydroxyl group with $n$-bromodecane in the presence of base, i.e. $\mathrm{K}_{2} \mathrm{CO}_{3}$, resulted in the formation of the long-chained compound (18). Finally, the desired ionic compound, i.e. (E)-1-decyl-4-[(4 decyloxyphenyl) diazenyl]pyridinium bromide (19), was prepared via alkylation of pyridine (Scheme 3). Also, POM and DSC studies revealed that compound 19 exhibits liquid crystalline properties from $117{ }^{\circ} \mathrm{C}$ to $134{ }^{\circ} \mathrm{C}$ during the heating cycle. While cooling, the compound showed a fan-shaped texture; this was designated as smectic A phase. In general, most of the ionic liquids exhibited smectic A phase. This method of synthesis is fast and simple; also, the target compound is an ionic liquid. Moreover, this compound can find applicability in hospitals and emergency units for the selective recognition of myoglobin in the near future.

The photoresponsive properties of halogen bonded liquid crystals (22a-j) in their liquid crystalline phases were studied by Chen et $a .^{15}$ During the synthesis of 22a-j (Scheme 4), azo pyridines (20a-e) were initially mixed with halogens [iodine (21a) or bromine (21b)]. The required starting materials, i.e. azo pyridines 20a-e, were prepared by referring to the report by Mallia et $a .^{16}$ Further, their studies revealed that the

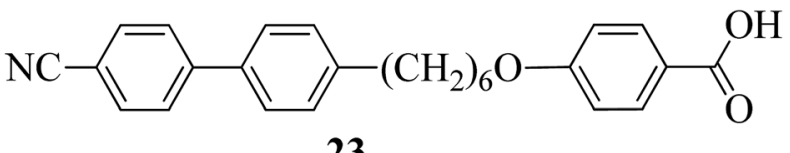

23

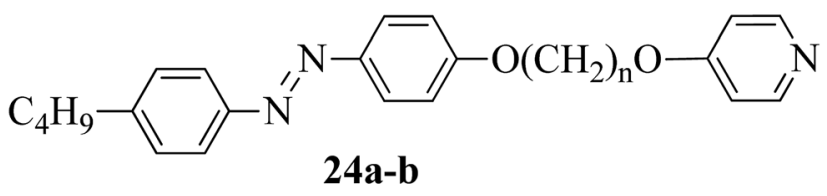

$$
\text { Where } n=5(\mathbf{2 4 a}) \text { or } n=6(\mathbf{2 4 b})
$$

Fig. 1 Structures of proton donor 23 and proton acceptors 24a-b.

synthesized azo pyridines (20a-e) are non-mesogenic. However, their halogen bonded complexes showed increased crystallization temperatures, and most of the complexes were liquid crystals. Furthermore, the azo pyridine-iodine complexes with lower chain lengths (i.e. 22a-b) were non-mesogenic in nature; however, as the chain length increased, smectic A phase appeared, and it was also noted that as the chain length increased in these complexes, the crystallization temperature decreased. However, all the azo pyridine-bromine (22f-j) complexes exhibited mesogenic properties (i.e. smectic A phase). Further, they studied the photoresponsive properties of these complexes through in situ observation by POM. Upon UV irradiation at $360 \mathrm{~nm}$, the mesogenic texture disappeared and a dark image was formed; hence, a photoinduced phase transition from liquid crystal phase to isotropic phase occurs in the iodine complexes (22a-e) but not in the bromine complexes $(\mathbf{2 2 f}-\mathbf{j})$. Hence, the observed phase transitions arise because of photoisomerisation of the prepared complexes from trans to cis configuration as the bent-shaped isomers destabilize the liquid crystal phases.

Paterson et al. ${ }^{17}$ prepared mixtures of mesogenic 6-( $4^{\prime}-$ cyanobiphenyl-4-yl)hexyloxybenzoic acid (23) with nonmesogenic compounds, i.e. 1-(4-butylazobenzene-4'-oxy)-5-(4-

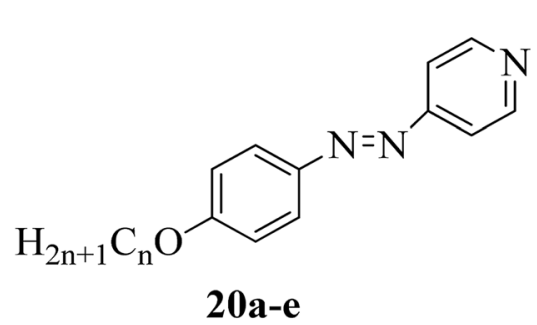

20a-e

\section{$+\mathrm{X}-\mathrm{X}$}

21a-b

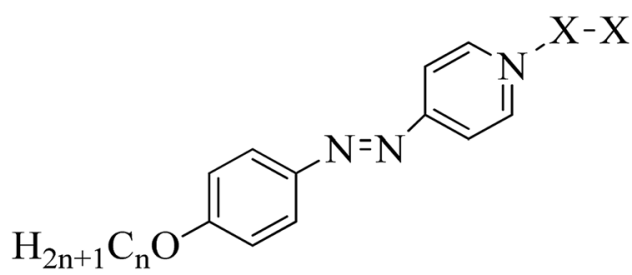

22a-j

Where $\mathrm{n}=6,8,10,12,14$. $\mathrm{X}=\mathrm{I}$ or $\mathrm{Br}$

Scheme 4 Preparation of halogen bonded liquid crystals (22a-j). 
<smiles>O=C(O)c1ccc(Oc2ccccc2)cc1</smiles>

25

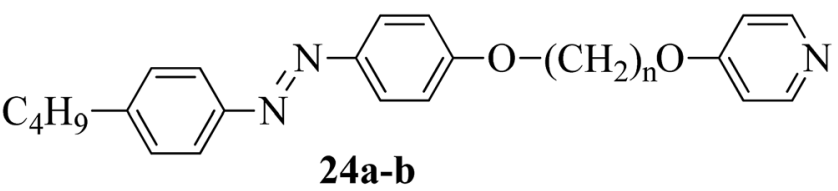

Where $n=5(24 a)$ or $n=6(24 b)$

Fig. 2 Structures of proton acceptors $24 a$ and $b$ and the structure of proton donor 25 .

oxypyridine)pentane (24a) or 1-(4-butylazobenzene-4'-oxy)-6-(4oxypyridine)hexane $(\mathbf{2 4 b})$, in a $1: 1$ molar ratio. They synthesized the mesogen (23) by referring to the article by Jansze et al. ${ }^{18}$ and two non-mesogenic units, i.e. $\mathbf{2 4 a - b}$, were synthesized by referring to the report by Wallage and Imrie. ${ }^{19}$ Fig. 1 presents the structures of proton acceptors $24 \mathbf{a}-\mathbf{b}$ and the structure of proton donor 23. The complexes of mesogenic and non-mesogenic compounds were prepared by dissolving equimolar amounts in chloroform solvent; the solvent was then evaporated and the products were dried. Both mixtures showed enantiotropic nematic phase. However, the evaporated twistbend nematic phase of $\mathbf{2 3}$ was quenched in both complexes. Further, the associated entropy change during the nematicisotropic transition for the $\mathbf{2 3 / 2 4 b}$ mixture and its transition temperature were found to be higher than those of the $\mathbf{2 3 / 2 4 a}$ mixture. The complete temperature ranges of the complexes were studied by Fourier transform infrared spectroscopy. It was revealed that this complex is not quantitatively formed but instead involves the $1: 1$ complex, free acid, and both open and cyclic acid dimers; hence, free $\mathbf{2 4 a}$ and $\mathbf{2 4 b}$ molecules exist in their prepared complexes. In all these mixtures, the molecules (average) exist in linear shapes, which helps to increase the elastic constant; hence, the stability of the twisted-bend nematic phases decreases.

In addition, Martínez-Felipe and Imrie ${ }^{20}$ prepared binary mixtures by reacting mesogenic 4-octyloxybenzoic acid (25) with either 1-(4-butylazobenzene-4'-oxy)-6-(4-oxypyridine)pentane (24a) or 1-(4-butylazobenzene-4'-oxy)-6-(4-oxypyridine)hexane (24b) in a $1: 1$ molar ratio. For synthesis, the starting materials, i.e. the two non-mesogenic units $(\mathbf{2 4 a}, \mathbf{b})$, were prepared by referring to the report by Wallage and Imrie $;^{19}$ mesogen 25 was obtained from a commercial source. Fig. 2 presents the structures of proton acceptors 24a-b (non-mesogenic) and the structure of proton donor 25 (mesogenic). The mesogenic and non-mesogenic compounds were initially dissolved in pyridine; the solvent was allowed to evaporate slowly, and the products were later dried in vacuum for $24 \mathrm{~h}$. From the XRD and POM studies, it is revealed that both the equimolar mixtures exhibit smectic A phase; also, in both cases, the nematic and smectic $\mathrm{C}$ phases of the mesogen (25) were quenched. However, the evenmembered complex was found to be well matched with the smectic A environment; hence, they observed a high transition temperature. Further, Fourier transform infrared spectroscopy revealed that the complexes are not quantitatively formed; instead, the prepared 1:1 mixtures contain free acid, both open and cyclic acid dimers and, hence, free $\mathbf{2 4 a}$ and $\mathbf{2 4 b}$ molecules.

He et al. ${ }^{21}$ synthesized nicotinate and isonicotinate derivatives (27a and $\mathbf{2 7} \mathbf{b}$, respectively) as proton acceptors and studied the effects of bent-shape or calamitic-shape structures on their mesogenic behavior. During the synthesis, nicotinic acid and DMF as solvent were combined and stirred. To this, thionyl chloride $\left(\mathrm{SOCl}_{2}\right)$ was added slowly at room temperature, and the reaction mixture was slowly stirred for about $24 \mathrm{~h}$. After completion of the reaction, the excess $\mathrm{SOCl}_{2}$ was removed in vacuum at $40{ }^{\circ} \mathrm{C}$. The resulting crude product was collected by filtration and washed with petroleum ether; the crude product was later dried in vacuum at room temperature to yield nicotinylchloride hydrochloride (26a). Immediately, it was taken in anhydrous THF; to this, 4-(4-propylcyclohexyl)phenol in pyridine was added. This reaction mixture was stirred for $24 \mathrm{~h}$ and was then filtered. The filtered product was further dissolved in hexane and activated carbon was added, followed by filtration and evaporation of the solvent to afford the hydrogen bond acceptor 4-(4-propylcyclohexyl)phenylnicotinate (27a). On the other hand, the donor, i.e. substituted 4-hydroxybenzoic acid (28a-c), was dissolved in $75 \%$ ethanol; then, $\mathrm{KOH}$ and KI were added, and the reaction mixture was stirred for $1 \mathrm{~h}$ at room temperature, followed by the addition of 1-bromo alkane and refluxing of the reaction mixture for $20 \mathrm{~h}$. Later, the solution was cooled and washed with petroleum ether, and the water layer was acidified using concentrated $\mathrm{HCl}$ solution to afford the precipitates of substituted 4-alkoxy-benzoic acid (29a-c(n)); then, the precipitates were filtered and recrystallized using $50 \%$ $\mathrm{v} / \mathrm{v}$ ethanol/water. To prepare the hydrogen bonded complexes of the nicotinate derivatives $(\mathbf{3 1 a}-\mathbf{c}(\boldsymbol{n}))$ and isonicotinate derivatives (30a-c(n)), the respective donor and acceptor compounds were dissolved in THF and heated at $40{ }^{\circ} \mathrm{C}$ for several minutes, followed by evaporation of the solvent and drying under reduced pressure for $24 \mathrm{~h}$ (Scheme 5). Also, the authors studied the effects of the terminal chain length and lateral fluorosubstituents on the prepared complexes. According to the authors, the length to breadth ratio decreased greatly when the structure of the hydrogen bonded liquid crystal changed to bent-shaped from calamitic; this led to decreases in the melting point and clearing point and also narrowed the temperature range of the mesophase. Similarly, substitution of fluorine showed a decreased length to breadth ratio, which led to a narrowed mesophase range.

Two series of supramolecular hydrogen bonded liquid crystal dimers were prepared by Al-Lami; ${ }^{22}$ the mesogenic properties of the dimers were studied, as well as their structureproperty relationships. The synthetic routes for the preparation of the hydrogen bonded complexes are shown in Scheme 6 . The author implemented two steps in the reaction route. The first step is the preparation of the hydrogen bond acceptor, i.e. $\alpha, \beta-$ bis(pyridine-4-yl-methylene)ethane-1,2-diamine (34), by 
<smiles>O=C(Cl)c1cccnc1</smiles><smiles>O=C(Cl)c1ccncc1</smiles>

4-(4-propylcyclohexyl)phenol, THF/pyridine

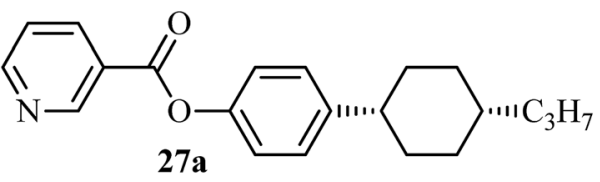<smiles>[X]c1cc(O)cc([X])c1C(=O)O</smiles>
28a-c 55b: $\mathrm{X}=\mathrm{H}, \mathrm{Y}=\mathrm{F}$ 55c: $\mathrm{X}=\mathrm{F}, \mathrm{Y}=\mathrm{F}$ 1. bromo alkane, $\mathrm{KOH}, \mathrm{EtOH}$; 2. $\mathrm{HCl}$

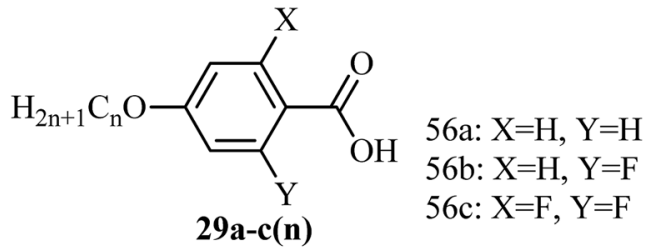

where $n=2,4,6,8,10,12$. 27b
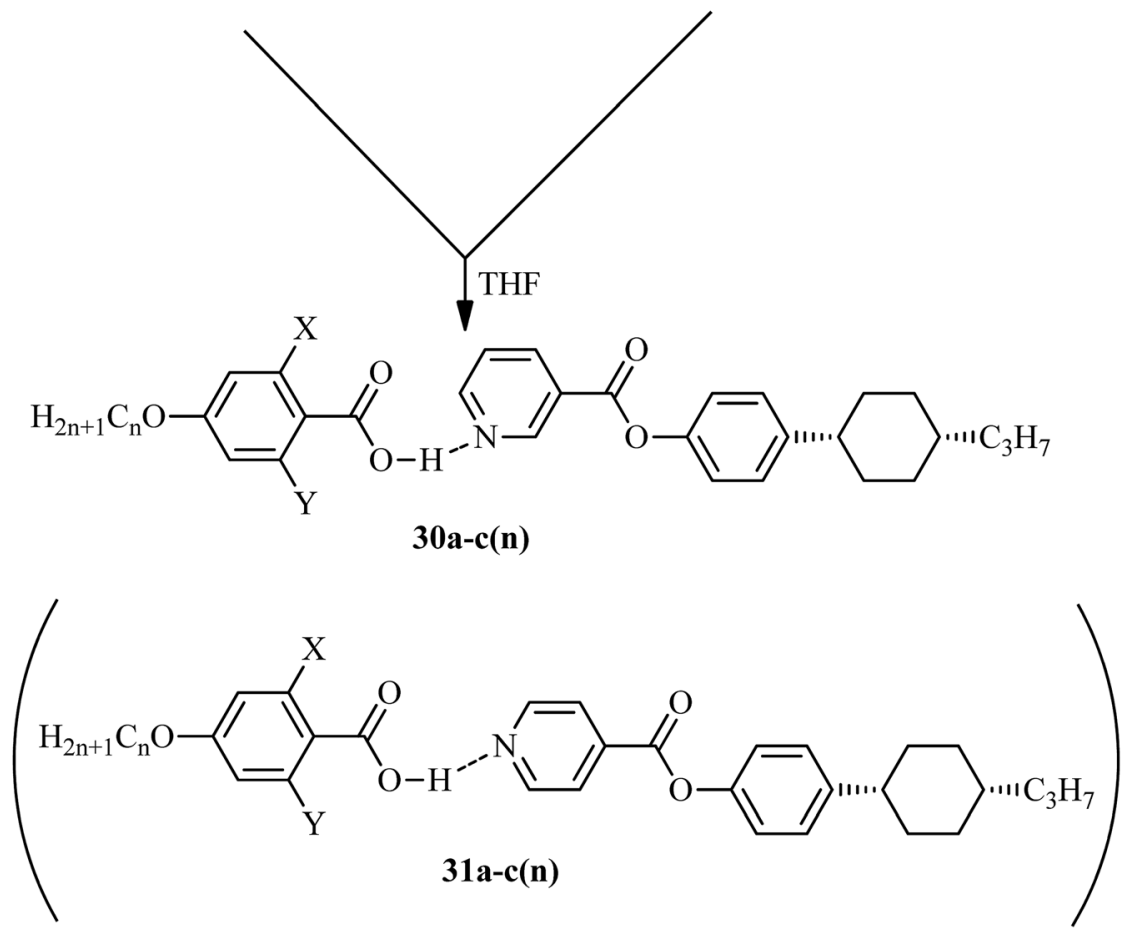

Scheme 5 Synthesis of hydrogen bonded complexes $30 a-c(n)$ and $31 a-c(n)$.

refluxing 4-pyridinecarboxaldehyde (32) with 1,2-diaminoethane (33) and a catalytic amount of glacial acetic acid in ethanol solvent for $2 \mathrm{~h}$; the obtained product is filtered and recrystallized using ethanol. The second step is the preparation of the hydrogen bond donors, i.e. 4-(4-alkoxybenzoyloxy)benzoic acid (39a-h) and 3,5-bis(4-alkoxybenzoyloxy)benzoic acid (37ah). These compounds were synthesized through the Steglich esterification method, wherein 4-alkoxy benzoic acid (36a-h) was reacted with 4-hydroxy benzoic acid or 3,5-dihydroxy benzoic acid in dichloromethane solvent followed by addition of the coupling reagent (i.e. 1,3-dicyclohexyl carbodiimide (DCC)) and catalyst (i.e. 4-dimethylaminopyridine). Further, the reaction mixture was stirred at room temperature for $24 \mathrm{~h}$. Finally, hydrogen bonded dimers $38 \mathbf{a}-\mathbf{h}$ and $\mathbf{4 0 a}-\mathbf{h}$ were prepared by dissolving the intermediates 34 and $\mathbf{3 7} \mathbf{a}-\mathbf{h}$ or $\mathbf{3 9 a}-\mathbf{h}$ in pyridine solvent, followed by evaporation of the solvent under various conditions for several days. First, the $\mathbf{4 0 a}-\mathbf{h}$ series exhibited nematic phase for shorter chain length compounds, i.e. 40ab $(n=2,3)$, and smectic A phase was noted for longer chain length compounds, i.e. $\mathbf{4 0 c}-\mathbf{h}(n=4$ to 8 and 12). This is due to the increased ratio of lateral to terminal attraction between the molecules with increasing chain length. As the chain length increased, the probability of layer arrangement during the melting process (crystal-liquid crystalline transition) also increased due to the weakened terminal attractions. In contrast, the second series of dimers (38a-h) exhibited nematic phase; 

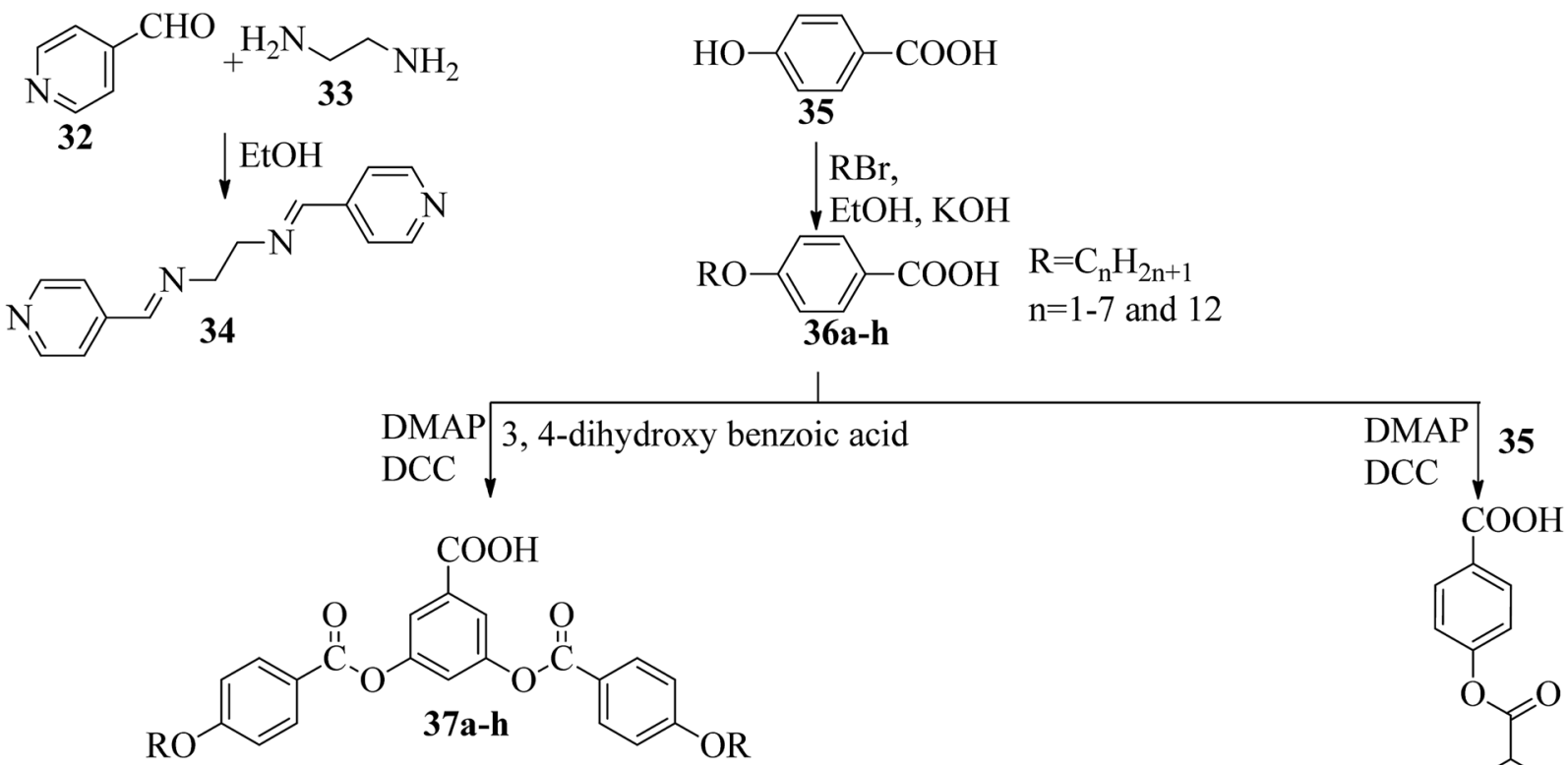

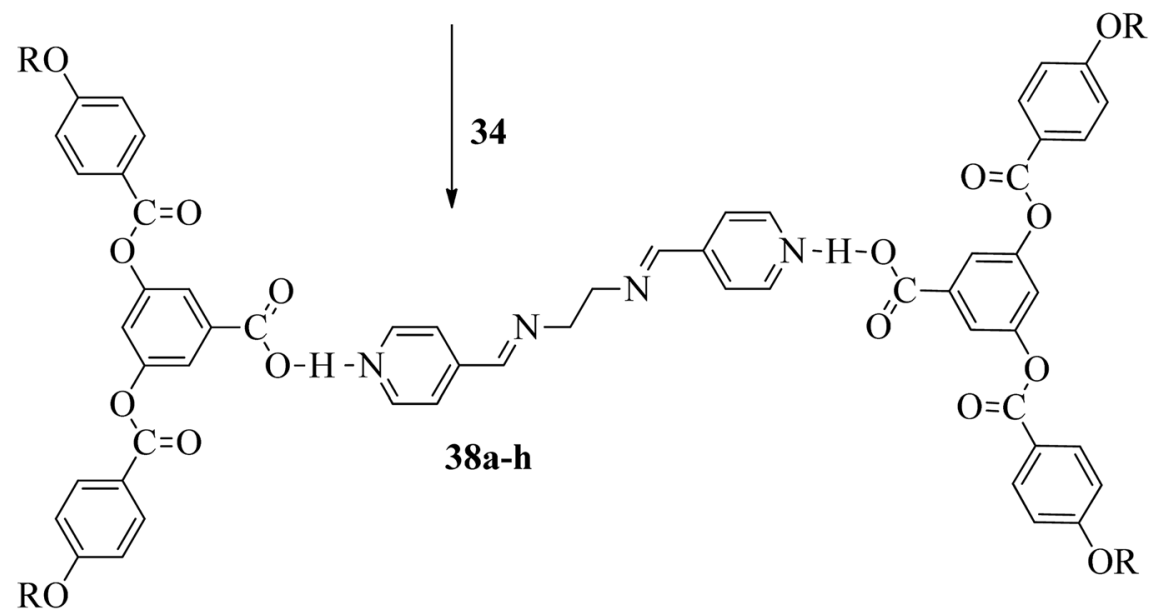<smiles>[R2]Oc1ccc(C(=O)Oc2ccc(C(=O)O)cc2)cc1</smiles><smiles></smiles>

Scheme 6 Preparation of hydrogen bonded complexes 38a-h and 40a-h.

this is due to the presence of four terminal alkoxy chains which were attached to the terminal phenyl ring but posed a smaller length/width ratio. Also, it was found that even-numbered chain lengths have higher transition temperatures than oddnumbered chain lengths. Further, their clearing temperatures and melting points were found to gradually decrease as the flexible alkoxy chain length increased, in which even-numbered members had slightly higher values. Furthermore, the decreased melting point and isotropic temperature were due to the increased terminal chain length; also, the number of possible conformations increased, which resulted in distortion of the cylindrical shapes of the mesogens.

Wei et $a l^{23}$ prepared a series of asymmetric hydrogen

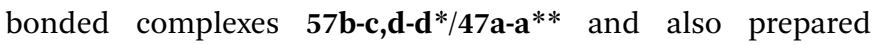
complexes with different molar ratios of proton donors (57b-c,d-

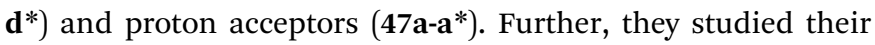
mesomorphic properties and compared them with their analogous covalent diads reported by Wei et al. ${ }^{24}$ Scheme 7 shows the synthetic route for the preparation of the hydrogen bonded complexes. Hydrogen bond donors $\mathbf{5 7} \mathbf{b} \mathbf{b}-\mathbf{c}, \mathbf{d}-\mathbf{d} *$ were synthesized by stirring $\mathbf{5 6} \mathbf{6}-\mathbf{c}, \mathbf{d}-\mathbf{d}^{*}$ and $10 \% \mathrm{Pd} / \mathrm{C}$ catalyst in THF solvent 

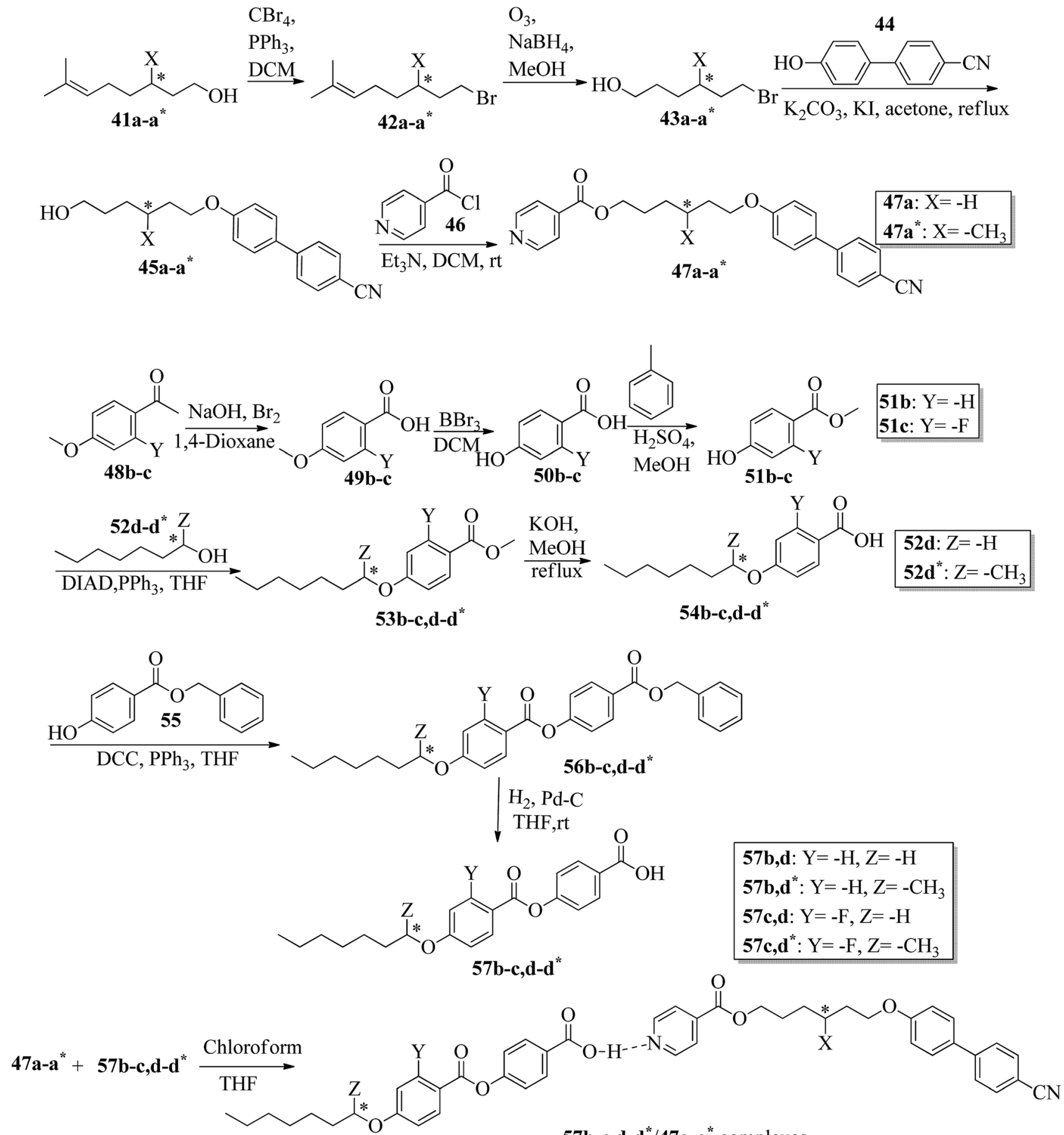

Scheme 7 Synthetic route of the $57 b-c, d-d * / 47 a-a *$ complexes.

under hydrogen atmosphere at room temperature overnight. After completion of the reaction, the catalyst was removed by filtration through Celite and washed thoroughly with THF. Later, the solvents were evaporated under reduced pressure. On the other hand, the hydrogen bond acceptors were prepared by dissolving isonicotinoyl chloride hydrochloride (46), compounds 45a-a* and triethylamine in dichloromethane (DCM) and stirring the reaction mixture at room temperature for $8 \mathrm{~h}$ under nitrogen. After completion of the reaction, the solvent was extracted with DCM and removed under reduced pressure to afford 47a-a*. Finally, the hydrogen bonded complexes were prepared by mixing appropriate molar ratios of hydrogen acceptors $\left(\mathbf{4 7} \mathbf{a}-\mathbf{a}^{*}\right)$ and hydrogen donors $\left(\mathbf{5 7} \mathbf{b}-\mathbf{c}, \mathbf{d}-\mathbf{d}^{*}\right)$ in THF solvent, followed by slow solvent evaporation. Further, the hydrogen bonded complexes $\mathbf{5 7 c , d} / \mathbf{d} / \mathbf{7} \mathbf{a}^{*}(1: 1 \mathrm{~mol})$ exhibited wide ranges of blue phase $\left(6.0^{\circ} \mathrm{C}\right)$ which became even 
<smiles>[Y][[Y]+]1ccc(-c2ccc(-c3ccc(-c4cc[n+]([R])cc4)s3)s2)cc1</smiles>

$\mathrm{X}_{1}: \mathrm{X}=\mathrm{I}$

$\mathbf{X}_{\mathbf{2}}: \mathrm{X}=\mathrm{NTf}_{2}$

Fig. 3 Structure of ionic liquid crystals $58 \mathrm{a}-\mathrm{eX}_{1-2}$.

wider $\left(13.2{ }^{\circ} \mathrm{C}\right)$ as the mole ratio changed to $3: 1$ due to the excess acid; dimers $\mathbf{5 7 c}, \mathbf{d}^{*}$ acted as chiral dopants. Comparing the blue phases of these complexes with the previously reported analogous covalent diads showed that the control of the blue phase majorly depends on the bent angle; if the bent angle lies between $132.1^{\circ}$ and $152.9^{\circ}$ in the molecular structure, it prefers to show blue phase. Because of the unsuitable bent angles in the $\mathbf{5 7} \mathbf{b}, \mathbf{d} / \mathbf{4 7} \mathbf{a}^{*}$ complexes $\left(162.0^{\circ}\right)$ and the covalent diads $\mathbf{5 7} \mathbf{b}, \mathbf{d}-$ $47 \mathrm{a}^{*}\left(126.5^{\circ}\right)$, they did not show blue phase. Further, complexes 57b,d/47a (1:1 mol) and 57b,d/47a* $(1: 1 \mathrm{~mol})$ have higher transition temperatures than complexes $\mathbf{5 7 c}, \mathbf{d} / \mathbf{4 7 a}(1: 1 \mathrm{~mol})$ and $\mathbf{5 7 c}, \mathbf{d} / \mathbf{4 7 \mathbf { a } ^ { * }}(1: 1 \mathrm{~mol})$, respectively, because the lateral fluoro substituent is larger than hydrogen. The large size of the lateral fluoro substituent and chiral center causes smaller $\pi-\pi$ interactions to occur in the mesogens; thus, the smectic A phase range was decreased by enlarging the $\mathrm{N}$ or $\mathrm{N}^{*}$ phase range. In addition, these complexes failed to show blue phases because of their smaller biaxial ratios and larger bent angles.

Cospito et al. ${ }^{25}$ prepared a series of thienoviologens, i.e. 4,4'(2,2'-bithiophene-5,5'-diyl)bis(1-alkylpridinium) $\mathrm{X}_{2}$, where $\mathrm{X}=\mathrm{I}$ $\left(\mathbf{5 8 a}-\mathrm{eX}_{1}\right)$ or $\mathrm{NTf}_{2}$, i.e. (bis(tri-fuoromethylsulfonyl)imide) (58a$\mathbf{e X}_{\mathbf{2}}$ ), and $m=8$ to 12 . The compounds (58a-eX $\mathbf{X}_{\mathbf{1}}$ ) were synthesized by suspending 5,5'-bis-(4-pyridyl)-2,2'-bithiophene in anhydrous chloroform under nitrogen atmosphere. To this stirred suspension, iodoalkane was added; the mixture was heated to $80^{\circ} \mathrm{C}$ for $24 \mathrm{~h}$, then cooled. The solvent was removed under vacuum and diethyl ether was added; then, the suspension was filtered, and the residue was washed with diethyl ether. Lithium triflimide was added to a stirred solution of viologen iodide in methanol and stirred for $15 \mathrm{~h}$ at room temperature; then, the solvent was removed, and the resulting mixture was added to water. The solid suspension was filtered and washed with water to afford $\mathbf{5 8 a -} \mathbf{e X}_{\mathbf{2}}$. Fig. 3 presents the structures of the ionic liquid crystals $\mathbf{5 8 a - e X _ { 1 - 2 }}$. Further, the study on this series revealed that when the chain length was small $(m=8)$, both series does not exhibited any mesophases; as the chain length increased, the series exhibited mesogenic properties. Comparing the mesomorphic properties of $58 \mathrm{a}-\mathbf{e X}_{1}$ and $58 \mathrm{a}-\mathbf{e X}_{2}$ indicated that $\mathbf{5 8} \mathbf{a}-\mathbf{e X}_{\mathbf{1}}$ have higher clearing points than $\mathbf{5 8 a -}-\mathbf{e X}_{\mathbf{2}}$. Moreover, compounds 58a-eX $\mathbf{X}_{2}$ have lower crystal-to-mesophase transition temperatures than $\mathbf{5 8} \mathbf{a}-\mathbf{e X}_{\mathbf{1}}$. These differences are mainly due to the larger size of NTf. Further, studies of the 58a$\mathbf{e X}_{\mathbf{2}}$ series indicated that the columnar arrangement was stable at lower chain lengths and lower temperatures, while calamitic behavior was seen at higher chain lengths and higher temperatures. This is mainly because the longer chains destabilize the dimers, leading to calamitic behavior. After the first heating scan of $\mathbf{5 8 e X}_{2}$, the columnar phase was lost because of the larger steric hindrance due to the longer chains. Studies also revealed that the transition temperature to the isotropic liquid state increased with increasing alkyl chain length. The mesophase range increased from $12{ }^{\circ} \mathrm{C}$ to $65^{\circ} \mathrm{C}$ with increasing alkyl chain length. 58a-eX $\mathrm{X}_{2}$ exhibited ionic liquid crystalline properties and multifunctional properties; they are also electro-chromic and exhibit strong fluorescence behaviour. Further, all the compounds are electro-active with n-type character, and their LUMO energy levels are very close to those of other organic ntype semiconductors. For all these reasons, these compounds have applicability in organic electronic applications as electronacceptor materials.

Fernandez-Palacio et al. ${ }^{26}$ synthesized halogen bonded fluorinated supramolecular liquid crystals and also studied their light-responsive properties. This was the first report on lightinduced and reversible crystal-to-isotropic phase transitions in supramolecular liquid crystals. They synthesized alkoxystilbazoles by referring to a previous literature report (i.e. Bruce et $a .^{27}$ ), while the iodoperfluorinated azobenzenes were synthesized by diazotization of 4-iodo-2,3,5,6-tetrafluoroaniline using nitrosonium tetrafluoroborate $\left(\mathrm{NOBF}_{4}\right)$ at $-30{ }^{\circ} \mathrm{C}$. After $1 \mathrm{~h}$, the mixture was stirred at room temperature with the relative alkoxybenzene in nitrogen atmosphere using acetonitrile solvent. Further, the mixture was extracted using dichloromethane $\left(\mathrm{CH}_{2} \mathrm{Cl}_{2}\right)$ and the solvent was removed under reduced pressure. In the case of the halogen bonded fluorinated supramolecular complexes, fluorine serves to increase the

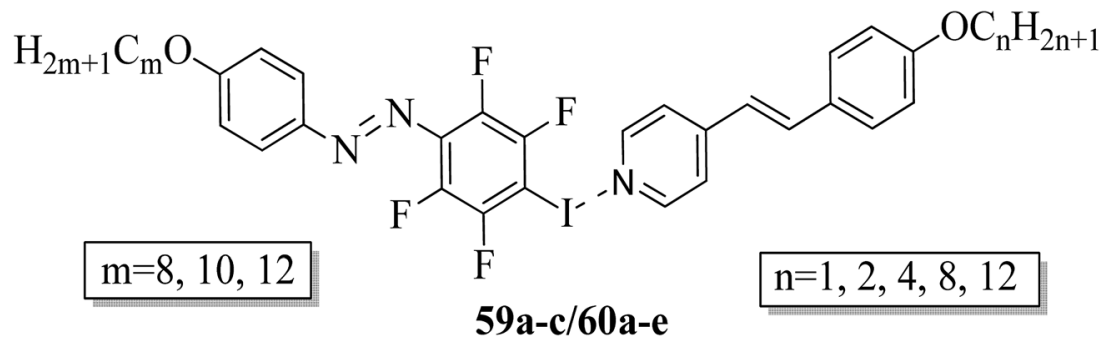

Fig. 4 Halogen bonded complexes 59a-c/60a-e. 


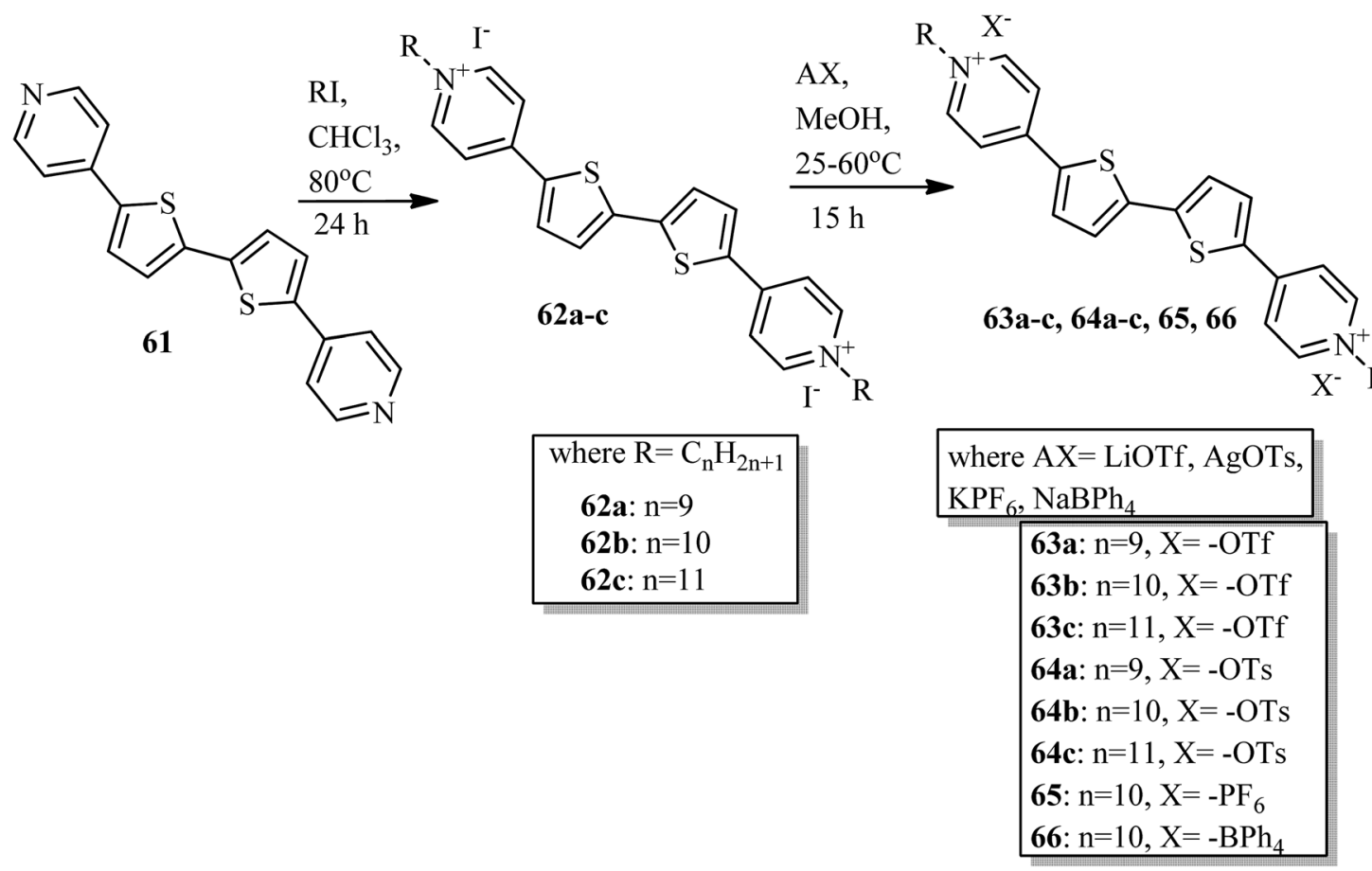

Scheme 8 Synthesis of thienoviologen salts.

lifetime of the cis-form of the azobenzene units and also strengthens the non-covalent interactions with the stilbazole molecules. Fig. 4 presents the prepared halogen bonded complexes (59a-c/60a-e). Further, enantiotropic liquid crystal phases were observed in all the prepared complexes except complexes 59c/60e, which were monotropic in nature. In all the other complexes, nematic phase was commonly observed, while smectic A phases were identified only in the complexes of the longest alkyl chains when attached at both ends. Most of these complexes exhibited broad temperature ranges of nematic phase and mesophase ranges greater than $30{ }^{\circ} \mathrm{C}$. They also noted that the onset temperatures for the mesophases can be suppressed by preparing mixtures of several complexes compared to single supramolecular entities. For example, they mixed 59c with 60b and 60e in a $1.0: 0.5: 0.5$ molar ratio and noted that the mixture exhibited a crystal-to-nematic transition at $79.5{ }^{\circ} \mathrm{C}$, which was $10{ }^{\circ} \mathrm{C}$ lower than that of pure $59 \mathrm{c} / 60 \mathrm{~b}$. Also, these complexes undergo fast and reversible transitions from isothermal liquid crystal-to-isotropic phase upon irradiation of UV light; also, they are photoresponsive complexes. They also undergo reversible crystal-to-isotropic transitions in addition to liquid crystal-to-isotropic phase transitions under UV light irradiation. Moreover, a clear reversible crystal-to-isotropic transition was observed within $30 \mathrm{~s}$ during $395 \mathrm{~nm}$ UV light irradiation. When the irradiation was ceased, recrystallization was observed in ca. 3 min through partial nematic phase; finally, homogeneous crystalline phase was formed. The partial nematic phase and significant delay were observed because of photoisomerization rather than a photothermal effect. Lightinduced changes in birefringence, absorption, and optical scattering analysis revealed that less than $4 \%$ of the mesogenic units in the cis-form were sufficient to cause the full liquid crystal-to-isotropic phase transition. Finally, it was concluded that these complexes have potential uses in the fabrication of supramolecular actuators and tunable photonic devices.

Veltri et $a .^{28}$ prepared thienoviologen salts and studied the effects of the counter anion and the chain length on their liquid crystal properties. 4, $4^{\prime}$ - $\left(2,2^{\prime}\right.$-bithiophene-5, $5^{\prime}$-diyl $)$ bis(1alkylpyridinium) was synthesized by referring to a previous report by Beneduci et al., ${ }^{29}$ and the anions were changed by counter ion exchange reactions, as reported in Scheme 8. During POM observation, the authors did not note any mesophase formation in compound 65. The observed nonmesogenity may be due to the stronger interaction of hexafluorophosphate anion with the cation, leading to the formation of stable crystalline phase compounds. Similarly, for compound 66 (containing tetraphenylborate anion), liquid crystal phase was not observed because of the large anion, which further destabilized the formation of the stable dimeric structure. However, triflate 63a-c and tosylate 64a-c exhibited liquid crystalline properties above $150{ }^{\circ} \mathrm{C}$; this was maintained up to $280{ }^{\circ} \mathrm{C}$ for the tosylate and $190{ }^{\circ} \mathrm{C}$ for the triflate, respectively. Smectic C phase was observed only in the complexes with shorter chain lengths (i.e. 63a and 64a). In contrast, compound 63b showed nematic phase during both the heating and cooling cycles, but compound 63c exhibited nematic phase only during the cooling cycle. In addition, columnar phase was observed in the compounds of triflate $63 \mathbf{a}-\mathbf{c}$ and tosylate $64 \mathbf{a}-\mathbf{c}$. Also, these compounds exhibited fluorescence properties in the bulk.

Further, He et al. $^{30}$ synthesized hydrogen bonded mesogens containing chiral carbons and their C-bonded mesogens by referring to the previous report by $\mathrm{He}$ et $a l . ;^{31}$ they also studied 


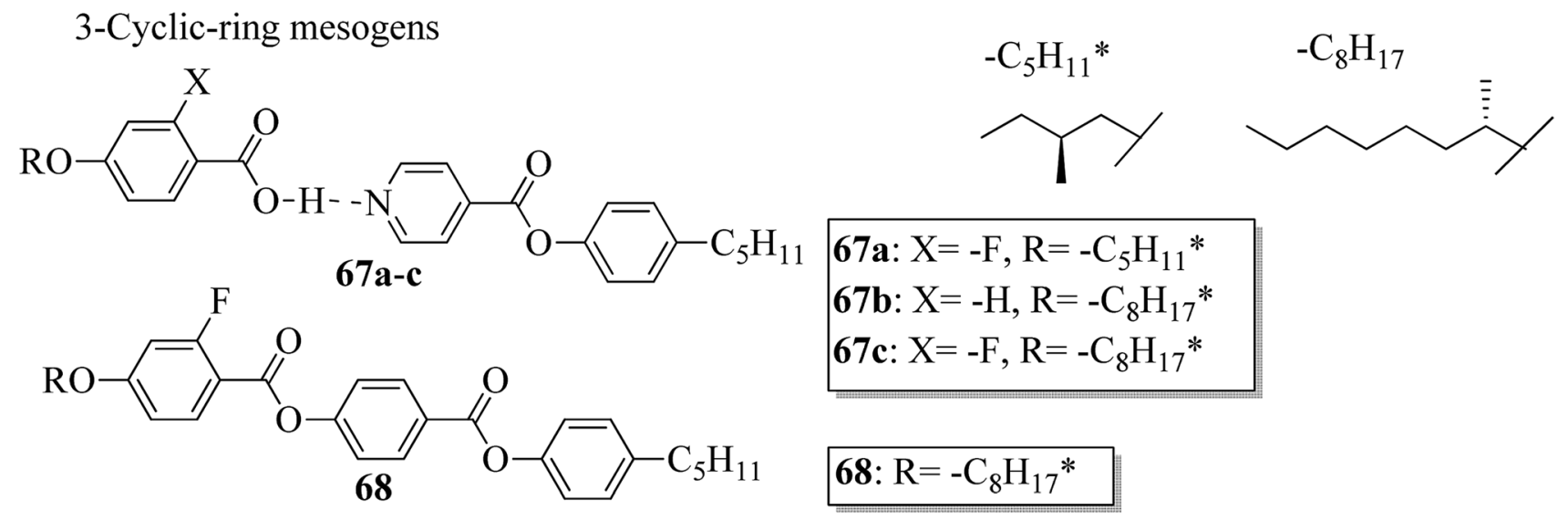

4-Cyclic-ring mesogens

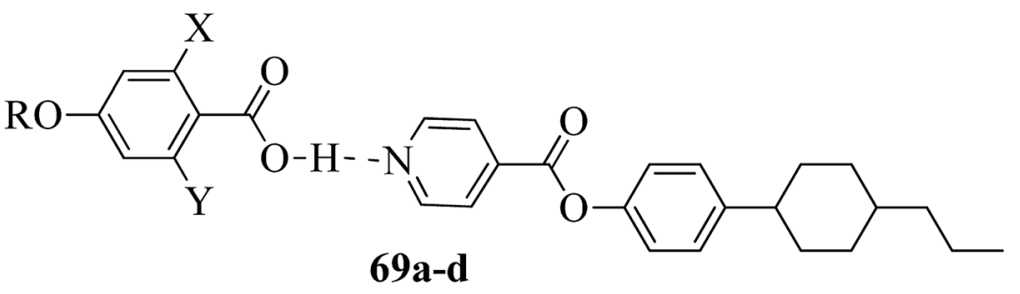

69a: $\mathrm{X}, \mathrm{Y}=-\mathrm{F},-\mathrm{H} ; \mathrm{R}=-\mathrm{C}_{5} \mathrm{H}_{11}{ }^{*}$

69b: $\mathrm{X}, \mathrm{Y}=-\mathrm{H},-\mathrm{H} ; \mathrm{R}=-\mathrm{C}_{8} \mathrm{H}_{17}$ *

69c: $\mathrm{X}, \mathrm{Y}=-\mathrm{F},-\mathrm{H} ; \mathrm{R}=-\mathrm{C}_{8} \mathrm{H}_{17}$ *

69d: $X, Y=-F,-F ; R=-C_{8} H_{17}$ *<smiles></smiles>

70

70: $\mathrm{R}=-\mathrm{C}_{8} \mathrm{H}_{17} *$<smiles>[R]Oc1ccc(-c2ccc(C(=O)O)cc2)cc1</smiles><smiles>C=CC(C)=CCC</smiles><smiles>CCCC1CCC(C(C)C)CC1</smiles>

71: $\mathrm{R}=-\mathrm{C}_{8} \mathrm{H}_{17} *$<smiles>[R20]c1ccc(C(=O)O)c(F)c1</smiles>

71<smiles>[R]Oc1ccc(-c2ccc(O)cc2)cc1</smiles>

17<smiles>[R]Oc1ccc(C(=O)Oc2ccc(-c3ccc(O)cc3)cc2)c(F)c1</smiles>

72: $\mathrm{R}=-\mathrm{C}_{8} \mathrm{H}_{17} *$

5-Cyclic-ring mesogens<smiles>[R20]c1ccc(-c2ccc(C(=O)O)cc2)cc1</smiles>

73: $\mathrm{R}=-\mathrm{C}_{8} \mathrm{H}_{17}{ }^{*}$

74a: $\mathrm{R}=-\mathrm{C}_{8} \mathrm{H}_{17}$ *

74b: $\mathrm{R}=-\mathrm{C}_{5} \mathrm{H}_{11}$ *

Fig. 5 Molecular structures of hydrogen bonded complexes 67-74.

the effects of the length of the terminal chains and the mesogenic core on the mesogenic properties of the compounds and their performance for extending the temperature range of the blue phase. Fig. 5 presents the molecular structures of the prepared complexes 67-74. They found that the hydrogen bonded complexes exhibited lower phase transition 


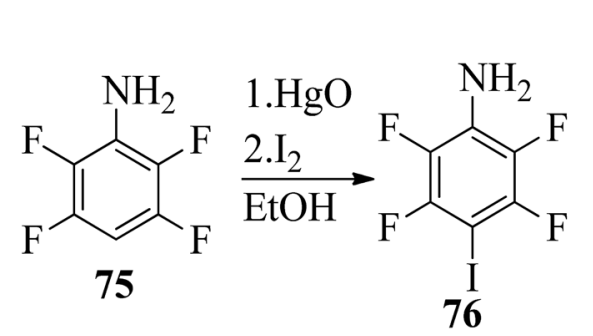

1. $\mathrm{SOCl}_{2}$<smiles>CN(C)c1ccc(C=Nc2c(F)c(F)c(I)c(F)c2N=Cc2ccc(N(C)C)cc2)cc1</smiles>

77

Scheme 9 Synthesis of $N$-benzylideneaniline (77).<smiles></smiles>

78

(b)

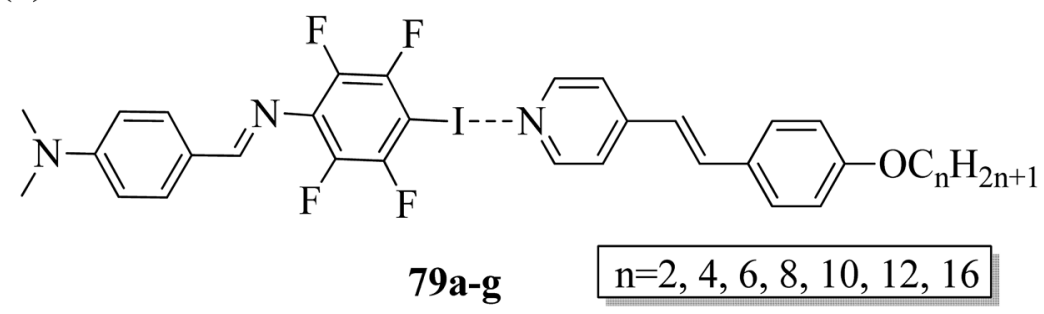

Fig. 6 (a) Structure of halogen bonded complex 78; (b) structure of halogen bonded complexes 79a-g.

temperatures than the analogous C-bonded compounds; also, the liquid crystal ranges were found to be narrower than those of the analogous C-bonded compounds. The complexes 67a and 67b exhibited monotropic nematic phases on cooling. However, their analogue 68 exhibited enantiotropic nematic phase. Complex 67c did not exhibit mesogenic properties. Compared to the 3-cyclic ring systems (67-68), the 4-cyclic ring systems (69-72) have high transition and clearing temperatures. Some of the hydrogen bonded complexes $(\mathbf{6 9 b}-\mathbf{d})$ exhibited smectic A phases; however, this was not observed in their C-bonded analogous compounds. Among the 4-cyclic ring system-based compounds, compound $\mathbf{7 1}$ exhibited a high nematic phase range. In the 5-cyclic ring systems, compound 74a exhibited nematic as well as smectic phases, and the remaining two complexes (74b and 73) exhibited only nematic phases. The hydrogen bonded complexes had lower phase transition temperatures because the blue phase range moved to room temperature. The blue phase range was not extended in<smiles></smiles>

Scheme 10 Preparation of halogen bonded complexes 82a-e. 
<smiles>CCCCCCC(C)Oc1ccc(C(=O)Oc2ccc(N=Nc3ccncc3)cc2)cc1N=Nc1ccc(OCCC(C)CCCC(C)C)cc1</smiles>

Hydrogen bond acceptor

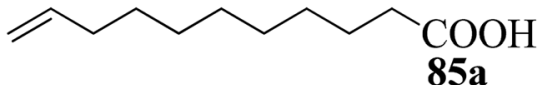<smiles>CCCCCCCCOc1ccc(C(=O)O)cc1</smiles>

85b<smiles>CCCCCCCCOc1ccc(/C=C/C(=O)O)cc1</smiles><smiles>CCCCCC[C@H](C)Oc1ccc(C(=O)O)cc1</smiles>

85d<smiles>CCCCCC[C@H](C)Oc1ccc(/C=C/C(=O)O[Na])cc1</smiles>

85e<smiles>CCCCCC[C@H](C)Oc1ccc2cc(C(=O)O)cc([Se])c2c1</smiles>

$85 f$
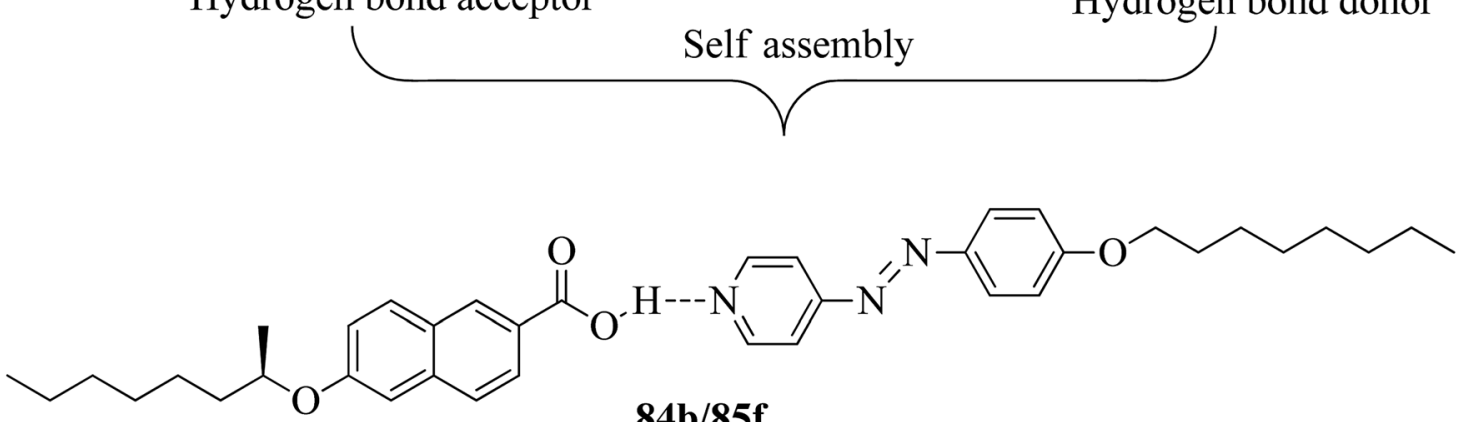

Fig. 7 Structures of azobenzene precursors, proton donors and complexes (84b/85f).

complex 67c due to the absence of mesogenic behavior in this complex. The widest blue phase range of about $12.9{ }^{\circ} \mathrm{C}$ was exhibited by the C-bonded 71-doped mixture compared to the<smiles>O=C(O)c1ccc(OCCOc2ccc(C(=O)O)cc2)cc1</smiles>

86

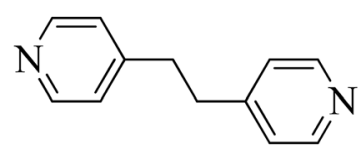

87

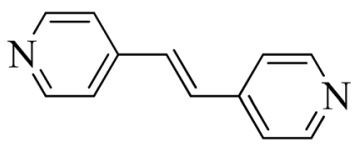

88
Fig. 8 Structures of proton donor 86 and proton acceptors 87 and 88 hydrogen bonded complex. In the complex with an achiral acceptor, the ability of chiral transfer from the chiral hydrogen bonded mesogen to the host liquid crystal is weak, which results in a narrower blue phase range compared to the $\mathrm{C}$ bonded analogues. Due to the weak ability of chiral transfer from the chiral hydrogen bonded mesogen to the host liquid crystal, the induced blue phase range of the C-bonded analog was wider than that of the hydrogen bonded complex with an achiral acceptor.

Wang et al. ${ }^{32}$ prepared halogen bonded complexes using $N$ benzylideneaniline 77 and 4-alkoxystilbazoles with pyridine as a proton acceptor moiety. They also prepared the halogen bonded co-crystal 78. By referring to the previous report by Bushuyev et al. ${ }^{33}$ they synthesized compound $\mathbf{7 6}$ by iodination of tetrafluoroaniline (75) in the presence of iodine $\left(\mathrm{I}_{2}\right)$ and 


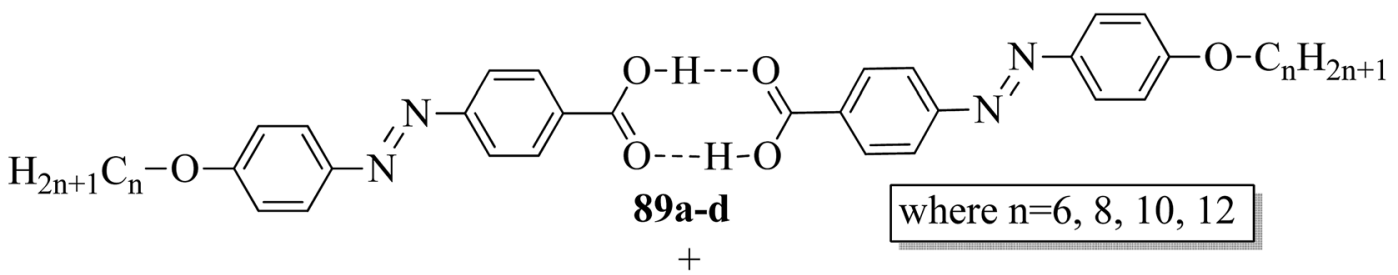

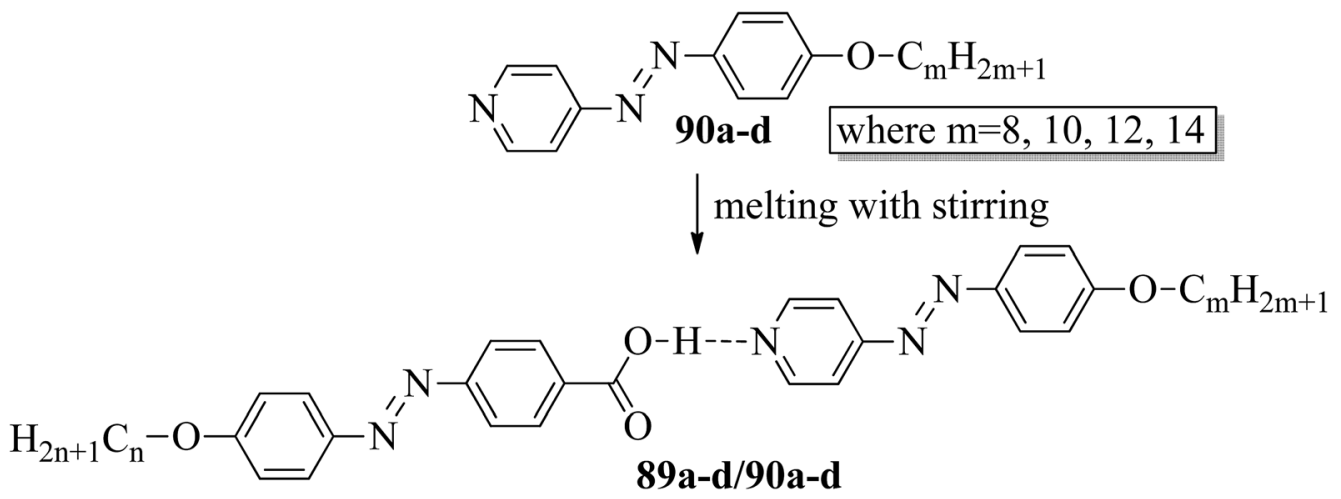

Scheme 11 Preparation of hydrogen bonded complexes $89 a-d / 90 a-d$.

mercuric oxide $(\mathrm{HgO})$ in ethanol solvent. They condensed 4dimethylaminobenzaldehyde and compound $\mathbf{7 6}$ in toluene solvent to synthesize $N$-benzylideneaniline 77 by referring to the previous report by Weiss and Pühlhofer ${ }^{34}$ (Scheme 9). Further, they synthesized the halogen bonded co-crystal $\mathbf{7 8}$ by dissolving a 2:1 ratio of compound 77 and bipyridine (BiPy) in THF solvent and allowing it to evaporate. In the crystal packing of compound 77, the two aromatic rings are not coplanar in alignment but are deviated towards each other. Additionally, there was no perceptible $\mathrm{C}-\mathrm{I} \cdots \mathrm{N}$ interaction between 4 -dimethylamino or imine and iodo-tetrafluorobenzene. However, it self-assembled into an unexpected fishbone-like assemblage by $\pi \cdots \pi$, C-I $\cdots \pi$ and the coordination of various intermolecular interactions. In addition, they found that halogen bonded co-

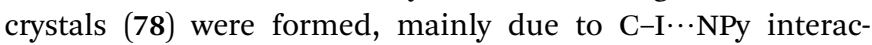
tions. Furthermore, the authors prepared the hydrogen bonded complexes $79 a-9$ by dissolving compound 77 and 4-alkoxystilbazoles in THF followed by slow evaporation of the solvent and drying under vacuum. Fig. 6 represents the structures of the halogen bonded complexes ( 78 and $\mathbf{7 9 a}-\mathbf{g}$ ). The DSC study revealed that liquid crystalline properties were not exhibited by the short chain complexes, i.e. 79a and 79b. This was ascribed to the fact that short chain 4-alkoxystilbazoles are not flexible enough. When the chain length increased to $n=6$ (i.e. 79c), nematic phase with a droplet texture was observed during POM investigation. However, the DSC study did not display a distinct liquid crystalline phase transition for this compound. It was also observed that both crystalline and nematic phase coexisted, and the liquid phase gradually transformed to the crystalline phase. This is presumably due to the very close transition temperatures of isotropic to nematic phase and of nematic phase to crystallization. Monotropic long-lived nematic phases were observed clearly as the 4-alkoxystilbazole chain length increased (79d, 79e, and 79f), and typical Schlieren textures were observed by POM. Even after several expeditions into the isotropic phase, the thermal behaviors were found to be reproducible. Especially, longer lived nematic phase was observed for 79e and reproducibly occurred at $25{ }^{\circ} \mathrm{C}$ before crystallization. Decreases in the mesophase ranges were observed on further increasing the chain length for $\mathbf{7 9 g}$, which exhibited a narrow range of nematic phase.

Kumar et $a .^{35}$ synthesized halogen bonded trimeric complexes using halogen bonded donors such as $\alpha, \omega$-diiodoperfluoroalkanes (81a-d) and 1,4-diiodotetrafluorobenzene (81e) and halogen bond acceptors such as methacrylate functionalized alkoxystilbazole (80). By referring to the literature report by Lin and Hendrianto, ${ }^{36}$ they synthesized the stilbazole methacrylate. Diiodoper-fluorocarbons (81a-e) and stilbazole methacrylate 80 were dissolved in THF solvent in $1: 2$ molar ratios, and crystallization from the solvent afforded the halogen bonded complexes (Scheme 10). The complexes obtained were yellow, whereas the parent compounds are white; this provides evidence for the successful formation of the complexes. This colour change was observed mainly because of the degree of charge shift from the pyridine nitrogen to the $\sigma$-hole on the iodine atom. Further, POM studies of the prepared 82a-e complexes revealed that all the parent compounds are nonmesogens, whereas the prepared $\mathbf{8 2} \mathbf{b}-\mathbf{e}$ complexes are mesogens. The halogen bonded complexes 82a-e have higher melting points than the pure halogen bond acceptor and donors. This provides evidence for the preferable formation of well-defined chemical species 82a-e rather than mechanical blends of the parent molecules. The prepared complexes 82a-e melted directly into isotropic liquids on heating. Monotropic smectic A phase was observed in complexes 82b-e on cooling from the isotropic phase; however, complex 82a degraded 


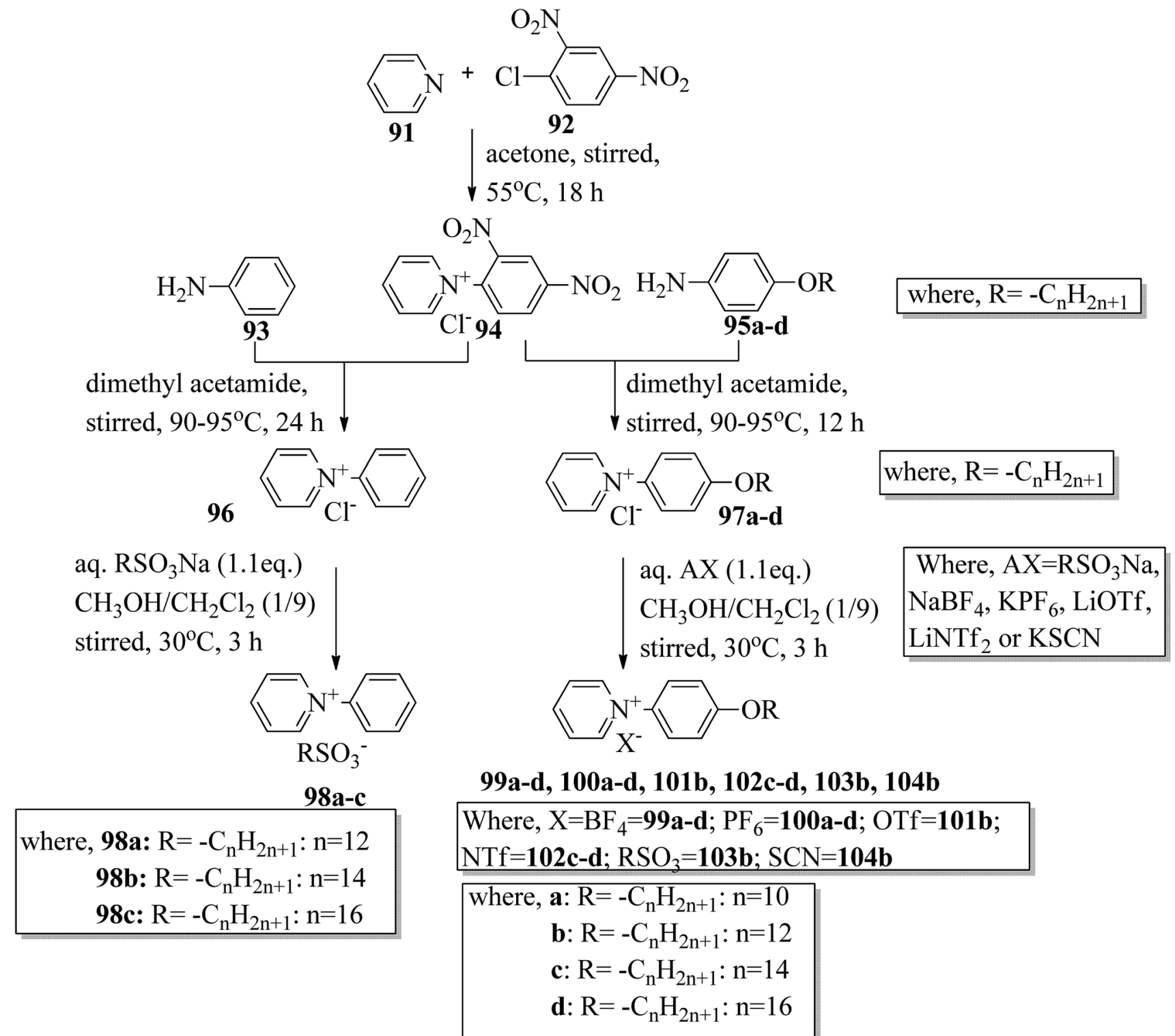

Scheme 12 Synthetic route for ionic liquid crystals 98-104.
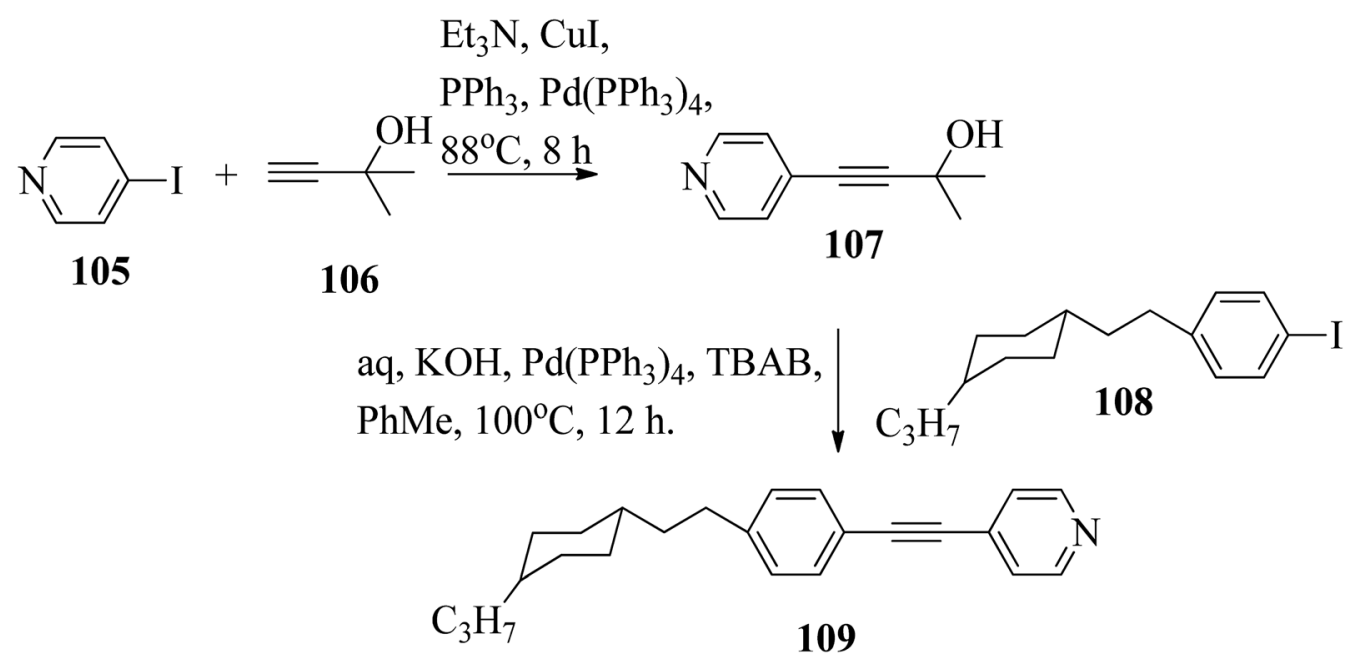

Scheme 13 Synthetic route for 109 

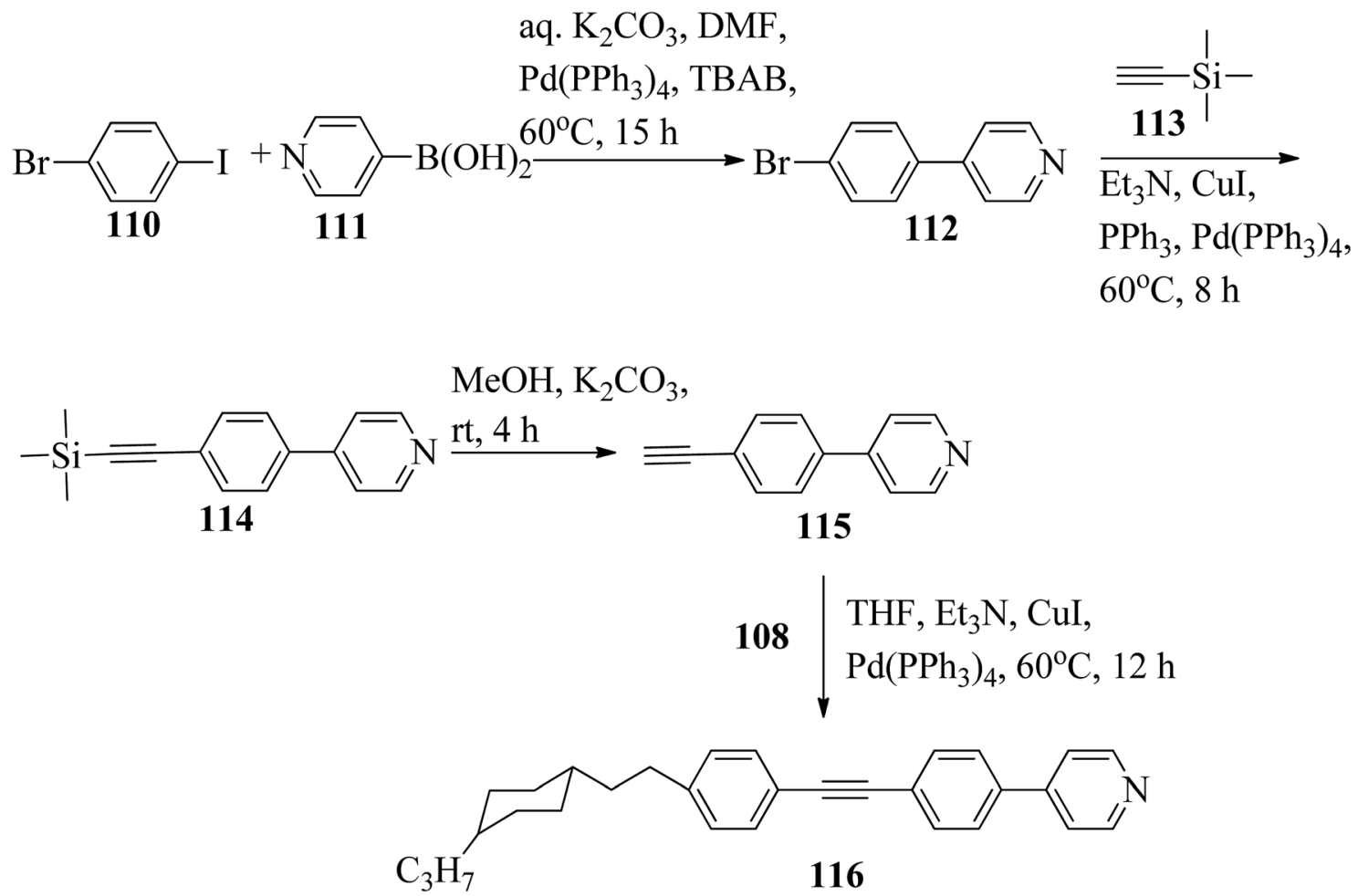

Scheme 14 Synthetic route for 116

promptly after melting. This result was observed because of the high volatility of the diiodoperfluoroethane molecule, which evaporated from the liquid mixture during heating. The complexes $\mathbf{8 2 b}$-e showed perfectly reproducible mesogenic behavior even after various periods in the isotropic liquid phase, and smectic A phases were noted across a range of $20^{\circ} \mathrm{C}$ to $30{ }^{\circ} \mathrm{C}$ ahead of the crystallization of the materials except for complex 82b, whose mesomorphic phase appeared to be long lived (up to $45{ }^{\circ} \mathrm{C}$ ). Because of the segregation between hydrocarbon chains and the fluorocarbons, monotropic smectic A phase was observed. Further, single crystal X-ray diffraction studies revealed that $\mathrm{N} \cdots \mathrm{I}$ XB interactions are mainly responsible for the self-assembly of the corresponding molecules $\mathbf{8 0}$ and 81a-e and displayed noticeable separations between the hydro-carbon molecules and perfluorocarbons; this ideally encourages the formation of lamellar phase in the liquid crystal state.

He et $a l .{ }^{37}$ synthesized pyridine-based azo compounds as hydrogen bond acceptors, i.e. 4-( $4^{\prime}$-pyridylazo)-4'-substituted benzoates or 4-(4'-pyridylazo $)-4^{\prime}$-alkoxybenzoates, with branched or straight terminals. Further, the authors prepared hydrogen bonded segregations using the synthesized hydrogen bond acceptors and different carboxylic acids. During synthesis, the 4-amino pyridine (83) and $\mathrm{HCl}$ were initially taken in a round bottom flask and cooled to $0{ }^{\circ} \mathrm{C}$ for some time, followed by the dropwise addition of sodium nitrate and phenol. Further, the reaction mixture was maintained in an ice bath and stirred for $0.5 \mathrm{~h}$. A yellow precipitate of 4-(4-hydroxyphenylazo)pyridine (84a) was obtained by maintaining the $\mathrm{pH}$ of the reaction mixture at 6 to 7 with the addition of $10 \mathrm{wt} \%$ aqueous $\mathrm{NaOH}$

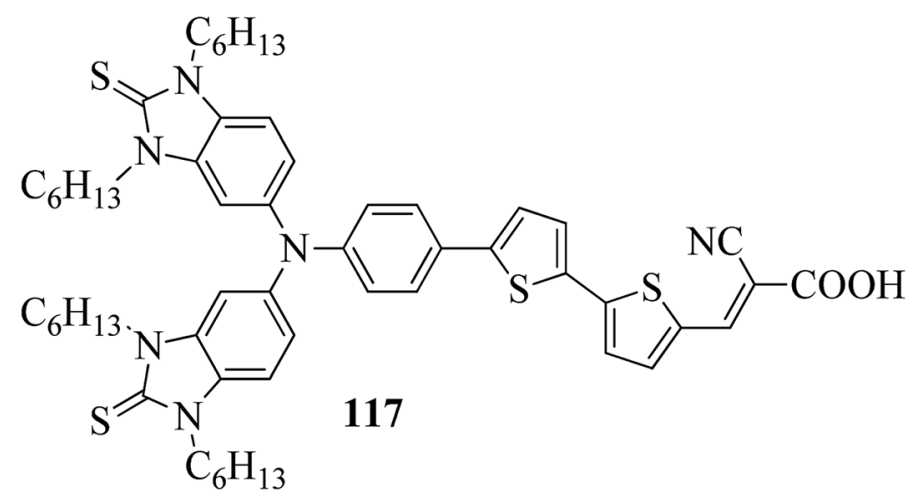

Fig. 9 Structure of 117 

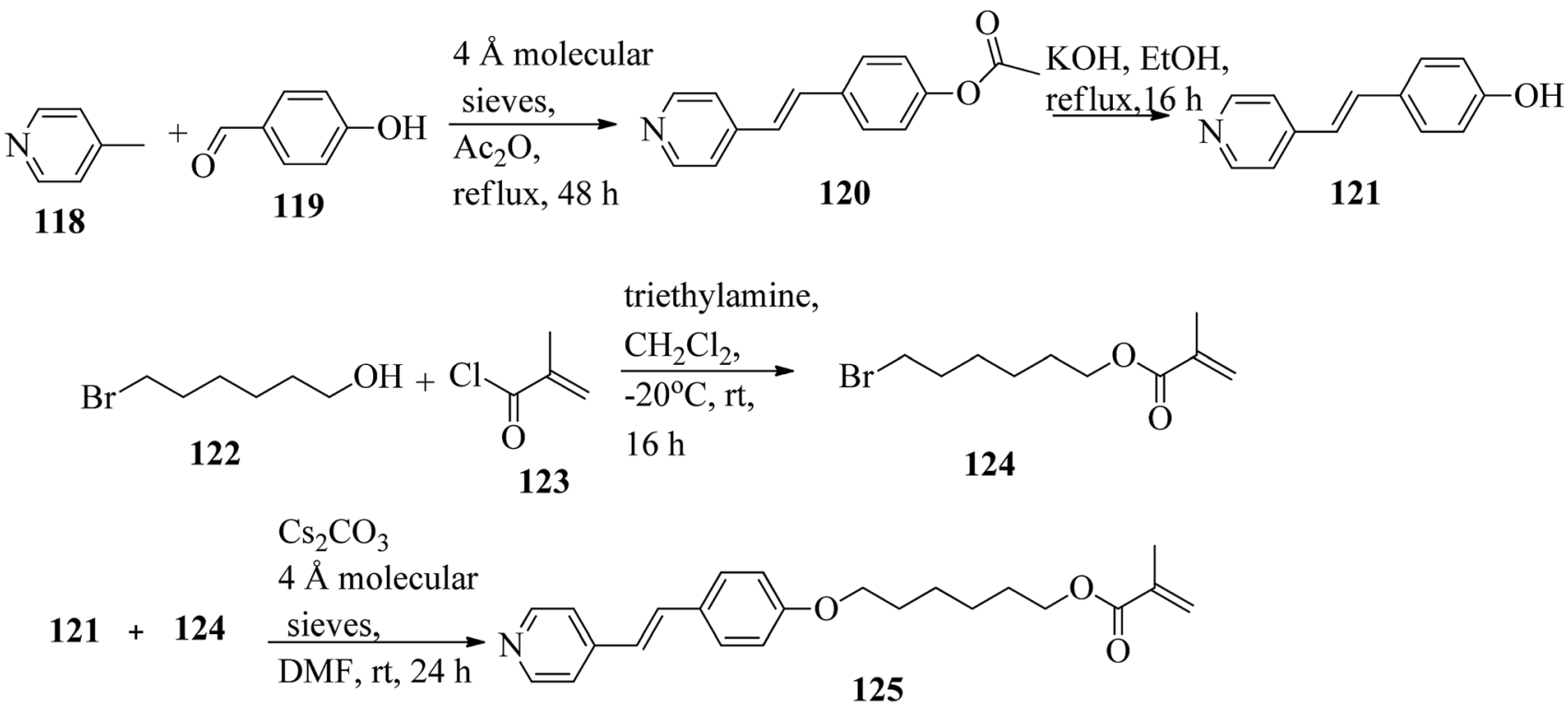

Scheme 15 Synthesis of 125

solution. Further, compound 84a, $\mathrm{K}_{2} \mathrm{CO}_{3}$, and $\mathrm{KI}$ were dissolved in acetone; to this, 1-bromooctane in acetone was added dropwise, and the mixture was refluxed for $16 \mathrm{~h}$ at $60{ }^{\circ} \mathrm{C}$. After completion of the reaction, the reaction mixture was poured into water, and the product was extracted using ethyl acetate. The solvent was removed using a rotary evaporator, and the crude product was then purified through silica gel column chromatography to afford pure 4-(4-octyloxyphenylazo)pyridine (84b). In a similar manner, they synthesized compounds 84c-e using 1-bromo-2-ethyl hexane and (R)-2-octanyl tosylate as starting materials. The structures of the azobenzene precursors and proton donors are presented in Fig. 7. The compounds $(S)$ 4-(octan-2-yloxy)benzoic acid, 4-(4-hydroxyphenylazo)pyridine, $N$-ethyl- $N$-(3-dimethylaminopropyl)carbodiimide hydrochloride and 4 -( $N, N$-dimethylamino)pyridine (DMAP) were dissolved in dichloromethane solvent and stirred at room temperature for $20 \mathrm{~h}$. After completion of the reaction, the reaction mixture was filtered and the solvents were removed under reduced pressure. Finally, the product 4-(4-hydroxyphenylazo)pyridine4-(octan-2yloxy)benzoate (84f) was purified through chromatography. Further, the hydrogen bonded complexes were prepared by dissolving appropriate amounts of hydrogen bond acceptors and donors in THF solvent followed by solvent removal using reduced pressure and drying in vacuum for $24 \mathrm{~h}$ at room temperature.

Liquid crystalline studies on these materials revealed that all the starting materials except $\mathbf{8 5 b}$ and $\mathbf{8 5 c}$ are non-mesogens; compound 85b exhibited smectic $\mathrm{C}$ phase and compound 85c exhibited nematic phase. The liquid crystalline properties of the prepared mixtures were studied using POM and DSC. Furthermore, the studies revealed that in the case of the precursors, the melting and clearing points increased with the introduction of terminal alkyl chains and decreased with increasing length of the rigid structures. Most of the complexes prepared using $84 \mathrm{~b}$ exhibited only smectic A phase. However, the $\mathbf{8 4 b / 8 5 b}$ and $\mathbf{8 4 b}$ / $\mathbf{8 5 f}$ complexes exhibited nematic phase in addition to smectic A phase. In the 84c-based complexes, only $\mathbf{8 4 c / 8 5 c}$ exhibited nematic phase; all the other complexes were non-mesogens. In the 84d-based complexes, nematic phase was observed in $\mathbf{8 4 d}$ / 85d, 84d/85c, and 84d/85f complexes, and the remaining complexes are non-mesogens. In the $\mathbf{8 4 e - b a s e d ~ c o m p l e x e s , ~ o n l y ~}$ 84e/85c exhibited nematic phase, and all the other complexes are non-mesogens. In the $\mathbf{8 4 f}$-based complexes, all the complexes exhibited nematic phase and twist ground boundary A phase except the 84f/85a complexes, which exhibited only smectic A phase. Finally, they concluded that mesomorphic states were observed in the straight terminal hydrogen bonded complexes, while the azo-derived complexes exhibited mesogenic behavior only with branched terminals. In general, when the proton donor is altered from a flexible chain to one or two rigid rings in its structure, the length-to-breadth ratio increases, which causes the extended mesogenic properties and increases in the phase transition points.

Carli et $a .^{38}$ prepared a series of hydrogen bonded liquid crystalline supramolecular copolymers. They synthesized the tetra(ethyleneglycxoy)bis-4-benzoic acid (86) by referring to the literature report by Greuel et al.; ${ }^{39}$ later, it was mixed with the proton acceptors 1,2-bis(4-pyridyl)ethene (88) and 1,2-bis(4pyridyl)ethane (87) and converted to a molten state for two minutes in nitrogen atmosphere, followed by cooling the mixtures to room temperature to yield the expected complexes. Fig. 8 presents the structures of proton donor $\mathbf{8 6}$ and the proton acceptors 87-88. Mesophase was observed when the rigid bis pyridyl (88) was hydrogen bonded; however, in the case of the structurally analogous flexible bispyridyl (87), mesophase was not observed. The complexes containing $\mathbf{8 7}$ and $\mathbf{8 8}$ as proton acceptors showed nematic phase at $75 \%$ addition of $\mathbf{8 8}$, and smectic $\mathrm{C}$ phase was observed below $50 \%$ addition of $\mathbf{8 8}$. As the 87 content increased, the lifetime of the liquid crystalline property decreases due to the enormous eutectic effect. Only 


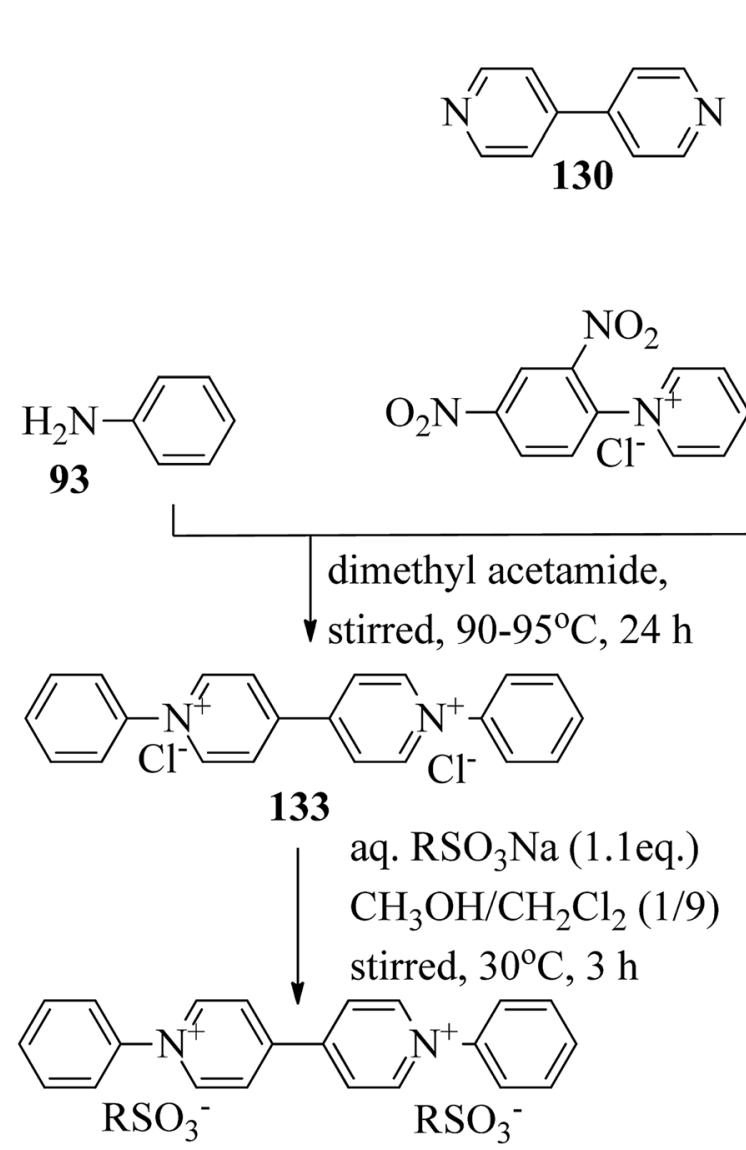

135a-c

$$
\begin{array}{r}
\text { where, a: } \mathrm{R}=-\mathrm{C}_{\mathrm{n}} \mathrm{H}_{2 \mathrm{n}+1}: \mathrm{n}=10 \\
\mathbf{b}: \mathrm{R}=-\mathrm{C}_{\mathrm{n}} \mathrm{H}_{2 \mathrm{n}+1}: \mathrm{n}=12 \\
\text { c: } \mathrm{R}=-\mathrm{C}_{\mathrm{n}} \mathrm{H}_{2 \mathrm{n}+1}: \mathrm{n}=14
\end{array}
$$<smiles></smiles>

acetone, stirred,

$\checkmark 55^{\circ} \mathrm{C}, 18 \mathrm{~h}$

$\mathrm{O}_{2} \mathrm{~N}$ 


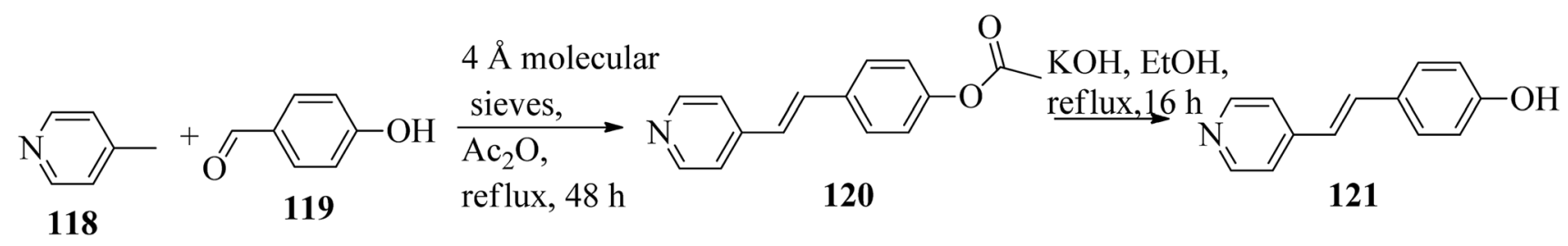<smiles>C=C(C)C(=O)OCCCCCCBr</smiles>

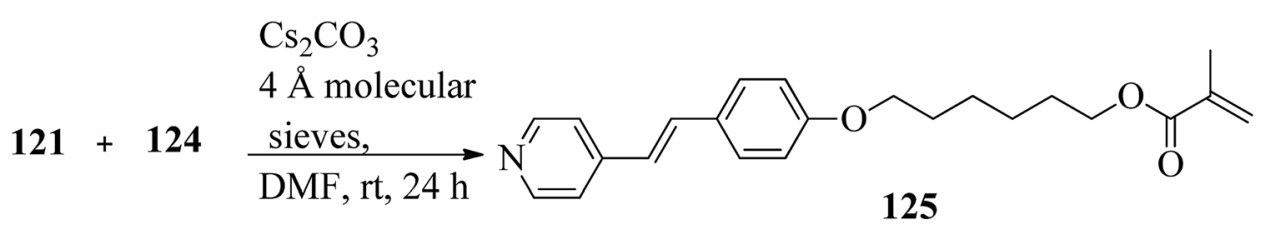

Scheme 15: Synthesis of 125.<smiles></smiles>

Fig. 10 Structure of hydrogen bonded complex 126 .

an intimate blend, followed by cooling to room temperature (Scheme 11). All the prepared complexes displayed two solid crystalline phases, followed by a smectic C phase and an observed broad nematic phase at the end; this was induced in these complexes, i.e. it was not observed in the cases of the pure acid components 89a-d. In all the samples, a more narrow

\begin{tabular}{|l|l|l|}
\hline Hydrogen bond donors & Hydrogen bond acceptor \\
\hline
\end{tabular}

Fig. 11 Structures of hydrogen bond donors 127 and 128a-b and acceptors 129a-e. 


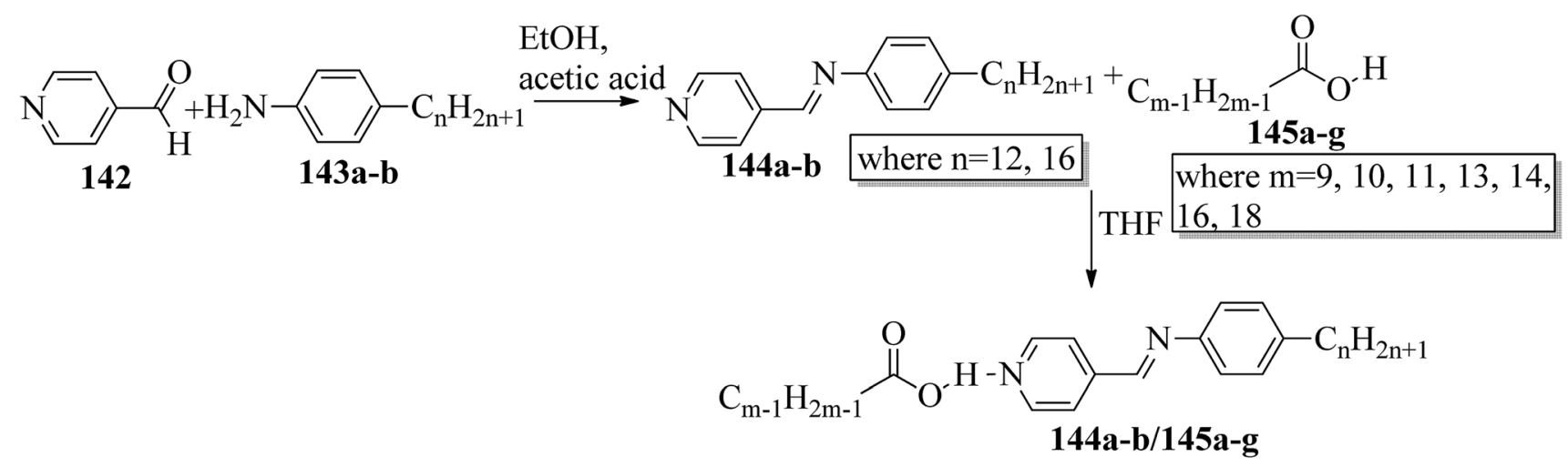

Scheme 17 Preparation of hydrogen bonded complexes $144 \mathrm{a}-\mathrm{b} / 145 \mathrm{a}-\mathrm{g}$.

range of smectic $\mathrm{C}$ phase than of nematic phase was observed. Additionally, an increase in $m$ moderately influenced the nematic phase range, whereas an increase in $n$ increased the nematic stability $\left(T_{\mathrm{N}-\mathrm{I}}\right)$. Also, the results revealed that for a specific value of $m$, the length of the alkoxy-chain in the acid derivatives $(n)$ moderately influences the melting temperatures of the supramolecular aggregations. Consequently, despite the fact that azopyridine compounds 90a-d do not display any mesophases, all the developed aggregations 89a-d/90a-d displayed induced nematic phases with relatively vast temperature spans, with a higher value of about $65.4^{\circ} \mathrm{C}$ for 89c/90a aggregation and a lower value of about $37^{\circ} \mathrm{C}$ for $\mathbf{8 9 b} / \mathbf{9 0 b}$ aggregation. Also, an increase in the alkoxy chain length of the acid complement $(n)$ causes an increase in the nematic transition enhancement $(\Delta T)$; therefore, the alkoxy chain length of the acid complement $(n)$ was found to have a greater effect on the nematic phase stability. Further, widening of the nematic phase was promoted by the increase in molecular anisotropy in the prepared supramolecular aggregations, in accord with previous reports (Ahmed et al. ${ }^{43}$ ). Furthermore, these studies revealed that the stabilities of smectic $\mathrm{C}$ and nematic phase increase as the mesogenic core length increases.

Wang et al. ${ }^{44}$ synthesized two series of ionic liquid crystals, i.e. $N$-phenylpyridinium derivatives (98-104). By studying previous literature (Michels et al., ${ }^{45}$ Zeghbib et al. ${ }^{46 a}$ Kuwabara et al. ${ }^{47}$ Kuo et al. ${ }^{48}$ ), they synthesized precursors such as $N$-phenyl pyridinium chloride 96 and $N$-(4-alkoxyphenyl)pyridinium chloride 97a-d. To substitute the chloride into different anions, they used methanol as a phase transfer medium. To prepare this, they dissolved the metal salt solution $\left(\mathrm{RSO}_{3} \mathrm{Na}, \mathrm{NaBF}_{4}, \mathrm{KPF}_{6}, \mathrm{LiOTf}, \mathrm{LiNTf}_{2}\right.$ or KSCN) in water, and this was added to a solution of 96 or $97 \mathbf{a}-$

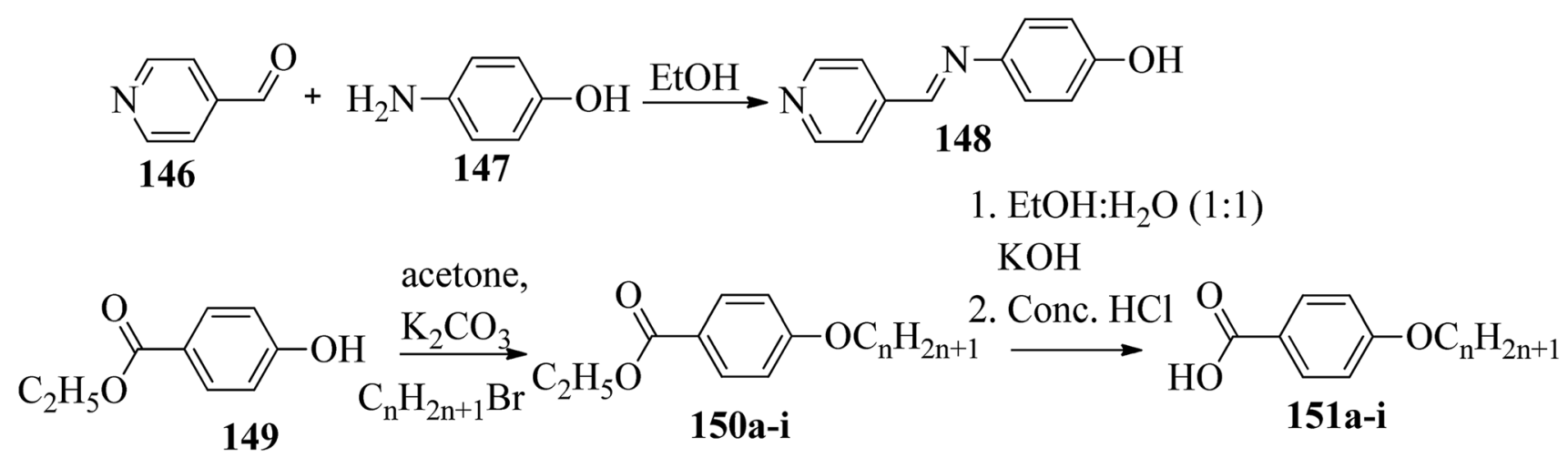

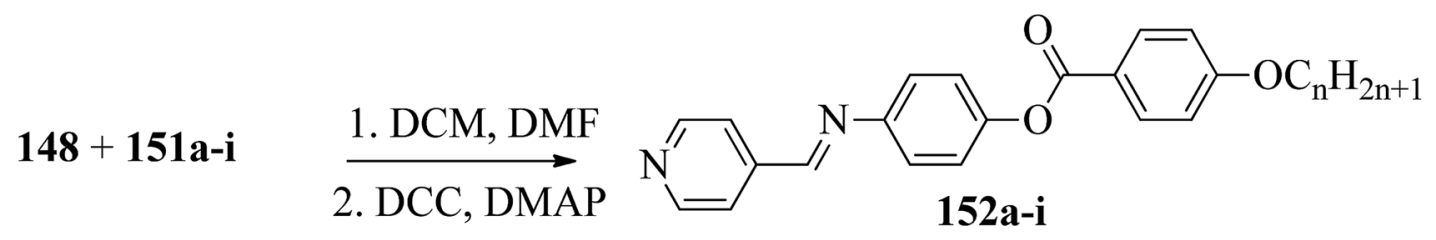

where, a: $n=2 ; \mathbf{b}: n=4 ; \mathbf{c}: n=6 ; \mathbf{d}: n=8 ; \mathbf{e}: n=10 ; \mathbf{f}: n=12 ; \mathbf{g}: n=14 ; \mathbf{h}: \mathbf{n}=16 ; \mathbf{i}: n=18$ 
<smiles>CCCCCCCCCC(=O)Oc1ccc(/N=C/c2ccncc2)cc1</smiles>

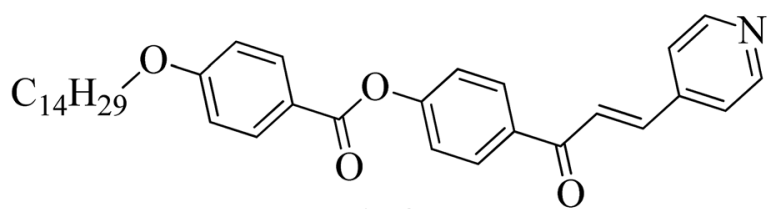
154

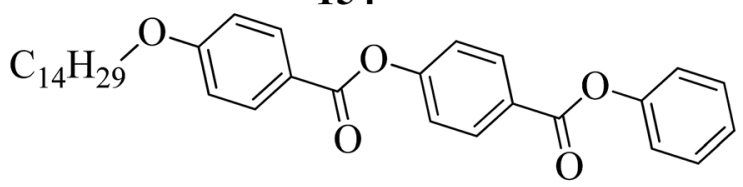

155

Fig. 12 Structures of 153, 154, and 155

d precursor in $\mathrm{CH}_{3} \mathrm{OH} / \mathrm{CH}_{2} \mathrm{Cl}_{2}$ (1/9). After $30 \mathrm{~min}$, the milky or cloudy solution became clear, followed by continuous stirring for $3 \mathrm{~h}$ at $30{ }^{\circ} \mathrm{C}$. Scheme 12 presents the synthetic route for the ionic liquid crystals 98-104. X-ray crystal structure analyses revealed that hydrogen bonds in the solid state induced bilayer lamellar or sandwiched head-to-head structures. In addition, the anion was embedded near the head group. All the compounds exhibited enantiotropic behavior except compounds 100a and 102c; these compounds exhibited monotropic behavior. DSC data of the compounds $(\mathbf{9 8 a}-\mathbf{c})$ revealed that the melting temperature remained at $91.0-107.0{ }^{\circ} \mathrm{C}$ while the clearing temperature $\left(T_{\mathrm{cl}}\right)$ increased with carbon chain length; i.e. $T_{\mathrm{cl}}=133.0^{\circ} \mathrm{C}$ $(\mathbf{9 8 a})<157.0{ }^{\circ} \mathrm{C}(\mathbf{9 8 b})<202.6{ }^{\circ} \mathrm{C}(\mathbf{9 8 c})$. As the chain length increases, the smectic $\mathrm{A}(\mathrm{SmA})$ phase range also increases; $\Delta T_{\mathrm{SmA}}=54.0^{\circ} \mathrm{C}(\mathbf{9 8 a})<77.0^{\circ} \mathrm{C}(\mathbf{9 8 b})<138.1{ }^{\circ} \mathrm{C}(\mathbf{9 8 c})$ during the cooling cycle. Further, the expanded temperature range of $\Delta T_{\mathrm{SmA}}=138.1^{\circ} \mathrm{C}$ in the derivative 98c indicates that this derivative has an almost ideal characteristic ratio of $d / l$, which is essential for mesophase formation. Also, the clearing temperature of compound $\mathbf{1 0 3 b}$ was higher than that of compound 98a; i.e. $T_{\mathrm{cl}}=133.0{ }^{\circ} \mathrm{C}<199.0{ }^{\circ} \mathrm{C}$. Here, the dodecyloxy chain-substituted compound $\mathbf{1 0 3 b}$ facilitated the mesophase formation. On the other hand, the mesophase range of $98 \mathrm{a}$ was narrower than that of $103 \mathbf{b}$, i.e. $\Delta T_{\text {meso }}=$ $85.4{ }^{\circ} \mathrm{C}>54.0{ }^{\circ} \mathrm{C}$ during the cooling cycle. Further, the DSC data of compounds 99a-d indicated that the melting temperature remained at $T_{\text {melt }}=83.7{ }^{\circ} \mathrm{C}$ to $88.6{ }^{\circ} \mathrm{C}$; at the same time, the clearing temperature increased with carbon chain length, i.e. $T_{\mathrm{cl}}=170.1{ }^{\circ} \mathrm{C}(\mathbf{9 9 a})<216.8{ }^{\circ} \mathrm{C}(\mathbf{9 9 b})<$ $252.9^{\circ} \mathrm{C}($ 99c $)<272.9^{\circ} \mathrm{C}$ (99d). As the chain length increases, the smectic A phase range also increases; $\Delta T_{\mathrm{SmA}}=116.0^{\circ} \mathrm{C}$ (99a) $<173.9{ }^{\circ} \mathrm{C}\left(\right.$ 99b) $<210.1{ }^{\circ} \mathrm{C}($ 99c $)<234.3^{\circ} \mathrm{C}$ (99d) during the cooling cycle. Among all the counter anions, the smallest temperature ranges of smectic A phase were observed in compounds 102c-d; $\Delta T_{\mathrm{SmA}}=11.0{ }^{\circ} \mathrm{C}(99 \mathrm{c})<26.5{ }^{\circ} \mathrm{C}(99 \mathrm{~d})$ during the cooling cycle. Also, they exhibited lower clearing temperatures than the other compounds; $T_{\mathrm{cl}}=56.8{ }^{\circ} \mathrm{C}(\mathbf{9 9 c})<$ $77.8{ }^{\circ} \mathrm{C}$ (99d). Due to the weaker coulombic forces in the unsymmetric and bulky $\mathbf{N T f}_{2}$ compounds, they exhibited lower clearing temperatures. The mesophase stability was significantly influenced by the type of counter anion; $\Delta T_{\mathrm{SmA}}$ $=221.3{ }^{\circ} \mathrm{C}(\mathbf{9 7 b})>213.1{ }^{\circ} \mathrm{C}(\mathbf{1 0 4 b})>173.9{ }^{\circ} \mathrm{C}(\mathbf{9 9 b})>85.4{ }^{\circ} \mathrm{C}$ $(\mathbf{1 0 3 b})>63.8^{\circ} \mathrm{C}(\mathbf{1 0 0 b})>41.4{ }^{\circ} \mathrm{C}(\mathbf{1 0 1 b})$. A significantly wider smectic A phase range was observed in compound 99d (i.e. $\Delta T_{\mathrm{SmA}}=234.3{ }^{\circ} \mathrm{C}$ ) which is broader than those of other reported ionic liquid crystals. Also, compound 102c is the first phenylpyridinium-based ionic liquid crystal. Among the prepared compounds, compound 104b showed room temperature mesophase.

Chen et al. ${ }^{49}$ synthesized compounds 109 and 116, which are dual functional molecules, and used them to observe rod-like liquid crystals on dye-sensitized solar cells. 4-(2-(trans-4- $n$-Propylcyclohexyl)ethyl)iodobenzene (108) was synthesized as described in a previous report by Chen et al., ${ }^{50,51}$ and organic dye 117 was synthesized by referring to a previous report by $\mathrm{Wu}$ et $a l .{ }^{52}$ 2-Methylbut-3-yn-2-ol (106) in triethylamine $\left(\mathrm{Et}_{3} \mathrm{~N}\right)$ was

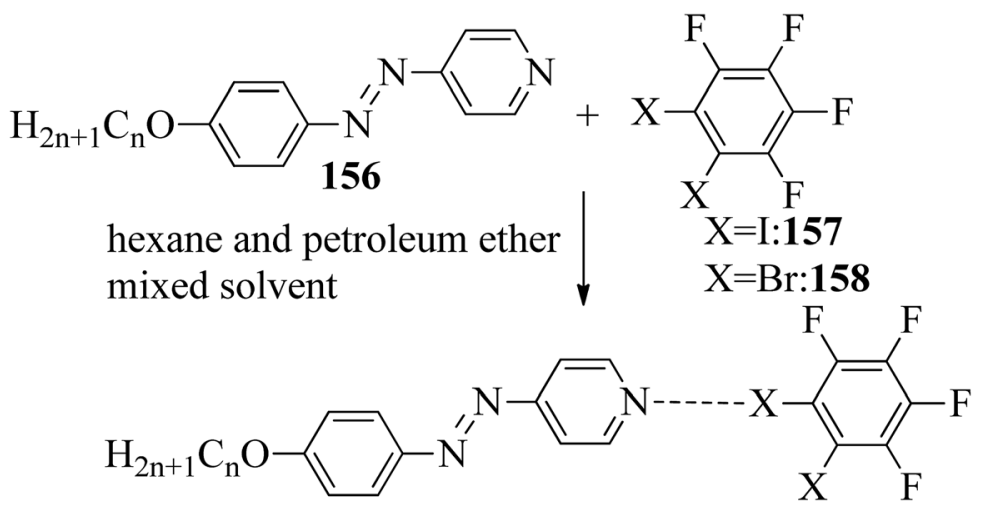

$\mathrm{X}=\mathrm{I}:$ 159a-k: $\mathrm{n}=8-18$

$\mathrm{X}=$ Br: 160a-f: $\mathrm{n}=10-15$

Scheme 19 Preparation of halogen bonded complexes 159a-k and 160a-f. 


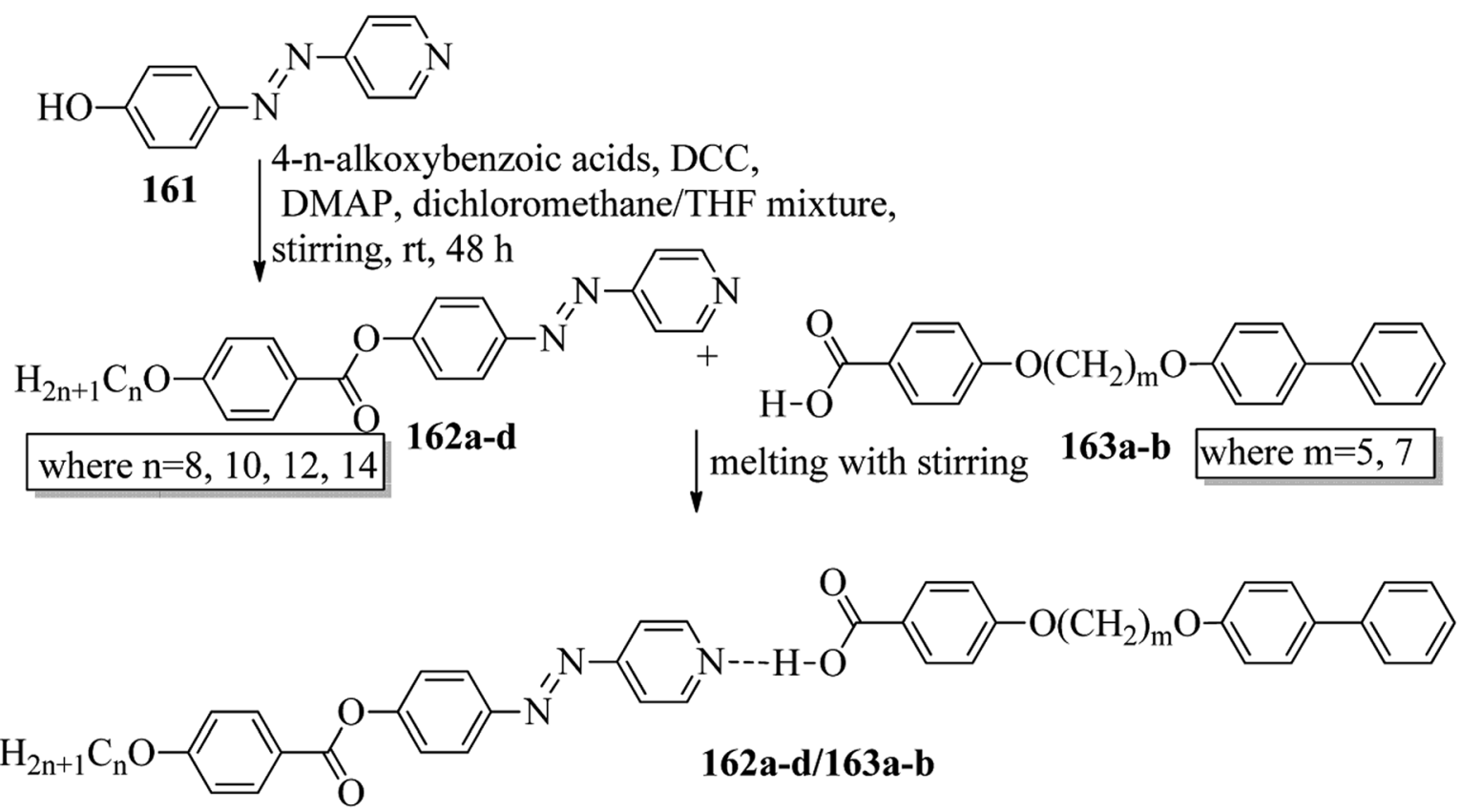

Scheme 20 Preparation of hydrogen bonded complexes $162 a-d / 163 a-b$.

added to DMF solvent containing 4-iodopyridine (105), CuI, $\mathrm{Pd}\left(\mathrm{PPh}_{3}\right)_{4}$, and $\mathrm{PPh}_{3}$ in dry $\mathrm{Et}_{3} \mathrm{~N}$ at room temperature. After completion of the reaction, the mixture was cooled and filtered; then, DCM was added to it, and the organic layer was washed using saturated ammonium chloride solution and dried using anhydrous $\mathrm{MgSO}_{4}$. Later, the solvents were evaporated to yield 2-methyl-4-(pyridin-4-yl)but-3-yn-2-ol (107). A mixture of $\mathrm{KOH}$, acetylenic alcohol 107, and TBAB in $\mathrm{PhMe} / \mathrm{H}_{2} \mathrm{O}$ at a ratio of $4: 1$ (v/v) was stirred for $30 \mathrm{~min}$ at $60{ }^{\circ} \mathrm{C}$ under nitrogen protection. Further, 108 and $\mathrm{Pd}\left(\mathrm{PPh}_{3}\right)_{4}$ were added, and the reaction mixture was stirred for $12 \mathrm{~h}$ at $100{ }^{\circ} \mathrm{C}$. After completion of the reaction, the solution was filtered over a pad of silica gel. The mixture was diluted using water and extracted using ethyl acetate. The combined organic phase was dried using $\mathrm{MgSO}_{4}$. After removal of solvent in vacuum, the product 4-((4-(2-(trans-4n-propylcyclohexyl)ethyl)phenyl)ethynyl)pyridine (109) was purified using silica gel column chromatography (Scheme 13). Further, 1-bromo-4-iodobenzene (110) and 4-pyridineboronic acid (111) were dissolved in DMF separately and mixed, then added to the $\mathrm{K}_{2} \mathrm{CO}_{3}$ solution. This reaction mixture was degassed for $10 \mathrm{~min}$ under nitrogen atmosphere; then, $\mathrm{Pd}\left(\mathrm{PPh}_{3}\right)_{4}$ was added and the mixture was stirred for $15 \mathrm{~h}$ at $60{ }^{\circ} \mathrm{C}$. After completion of the reaction, the solvent was removed by rotary evaporation and the compound was dissolved using $\mathrm{CH}_{2} \mathrm{Cl}_{2}$ solvent and washed with water and brine solution. Then, the organic layer was dried using magnesium sulphate, concentrated and purified using chromatography to afford pure 4-(4-bromophenyl)pyridine (112). Ethynyltrimethylsilane (113) in $\mathrm{Et}_{3} \mathrm{~N}$ was added to DMF solvent containing 4-(4-bromophenyl)pyridine (112), CuI, $\mathrm{Pd}\left(\mathrm{PPh}_{3}\right)_{4}$, and $\mathrm{PPh}_{3}$ in dry $\mathrm{Et}_{3} \mathrm{~N}$ at room temperature. The reaction mixture was stirred at $60{ }^{\circ} \mathrm{C}$ for $8 \mathrm{~h}$ under nitrogen atmosphere. After completion of the reaction, the mixture was cooled and filtered; then, DCM was added, and the organic layer was washed using saturated ammonium chloride solution and dried using anhydrous $\mathrm{MgSO}_{4}$. The solvents were evaporated to yield 4-(4-((trimethylsilyl)ethynyl)phenyl)pyridine (114). Further, compound 114 and potassium carbonate were dissolved in methanol and stirred for $4 \mathrm{~h}$. After the completion of the reaction, DCM extraction was performed, followed by washing with water and brine. Further, the organic layer was dried, followed by evaporation of the solvent under reduced pressure to yield 4-(4-ethynylphenyl) pyridine (115).

In a two-way flask, compound 115 and $\mathrm{Et}_{3} \mathrm{~N}$ were dissolved in THF, and the solution was bubbled with nitrogen. 4-(2-(trans-4$n$-Propylcyclohexyl)ethyl)iodobenzene (108), $\mathrm{Pd}\left(\mathrm{PPh}_{3}\right)_{4}$, and CuI were taken in another two-way flask containing THF solvent and covered with nitrogen; to this, the previously prepared mixture was added dropwise. This reaction mixture was stirred for $12 \mathrm{~h}$ at $60{ }^{\circ} \mathrm{C}$. Saturated ammonium chloride was added to remove CuI. Using water, the reaction mixture was diluted and extracted with ethyl acetate. Using $\mathrm{MgSO}_{4}$, the combined organic layer was dried followed by evaporation of solvent in vacuum to yield 4-(4-((4-(2-(trans-4-n-propylcyclohexyl)ethyl)phenyl)ethynyl) phenyl)pyridine (116), as shown in Scheme 14. Later, the crude product was purified by silica gel column chromatography. Using POM, DSC, and XRD experiments, the mesogenic behavior of 109 and 116 was studied. POM observation revealed that 109 and 116 exhibit nematic mesophase in the cooling scan. Liquid-like ordering with a diffuse peak in the wide-angle region with an intermolecular distance of $4.6 \AA$ was observed when 109 was cooled from isotropic phase. Compound 109 exhibited monotropic phase (i.e. it exhibited nematic phase only in the cooling cycle). Compound 116 exhibited 

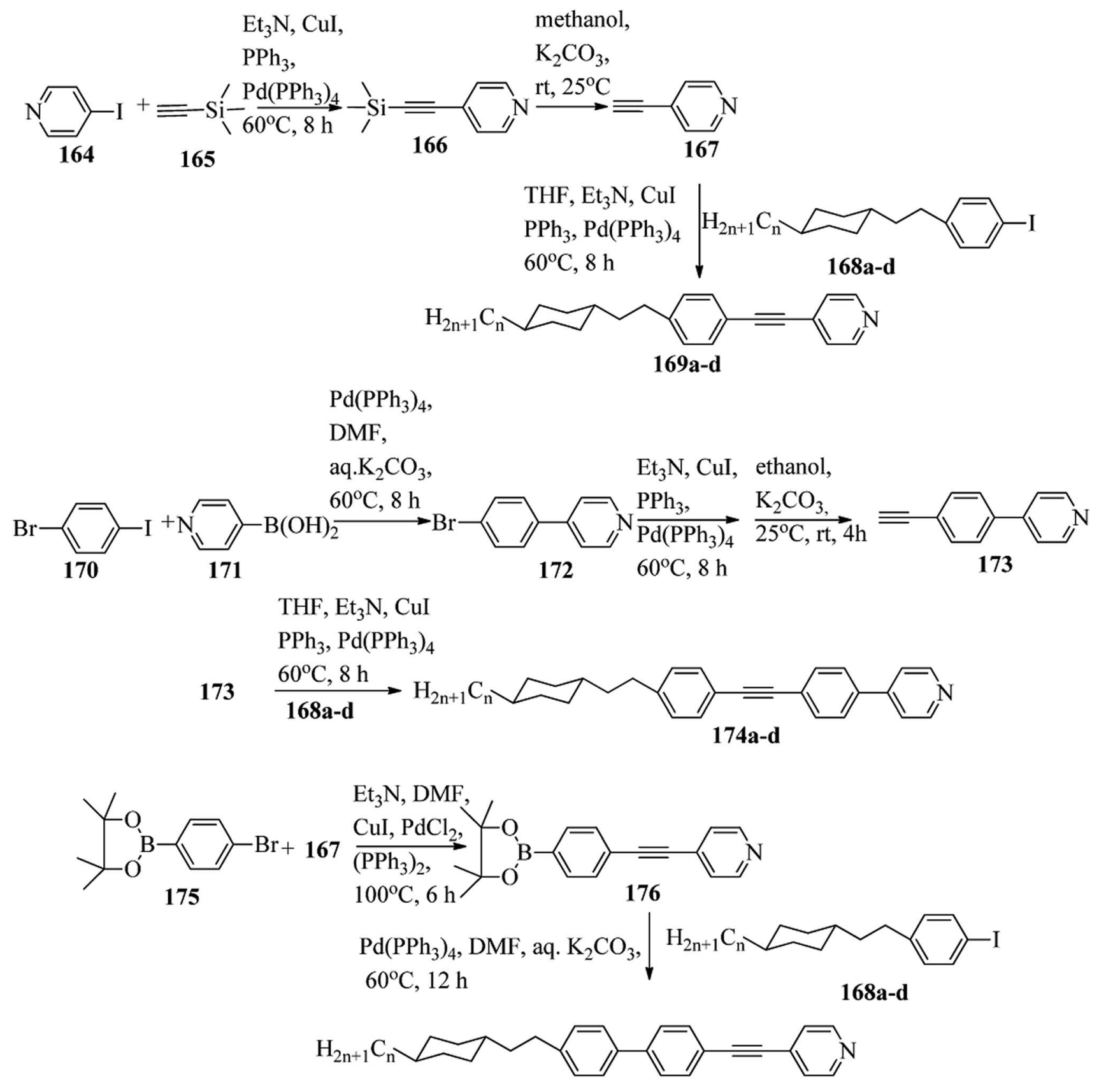

$177 \mathbf{a}-\mathbf{d}$

where $\mathrm{n}=2,3,4,5$

Scheme 21 Synthesis of $177 a-d$.

enantiotropic nematic phase (i.e. it exhibited nematic phase in both the heating and cooling cycles). On the other hand, these two mesogens exhibited different effects on the photoconversion efficiency and long term stability of co-sensitized dye-sensitized solar cells 109/117 and 116/117. Fig. 9 presents the structure of 117. The molecular structures of 109 and 116 show steric repulsion of molecules and inter-annular twisting, which is due to the additional phenyl ring on $\mathbf{1 1 6}$. Hence, the $\pi-$ $\pi$ packing is minimized and nematic mesophase is induced despite the lengthening of the molecular axis.

Mulder et al. ${ }^{53}$ prepared hydrogen bonded hetero-dimers for the fabrication of a cationic nanoporous smectic liquid crystal network (LCN). The preparation procedures depend on supramolecular aggregations composed of non-reactive benzoic acid rigid materials hydrogen bonded with a pyridyl-bearing reactive mesogen. By referring to procedures given in reports by Shaw et $a l .{ }^{54}$ and Chiang et al. ${ }^{55}$ they synthesized the compound 4- 
<smiles></smiles>

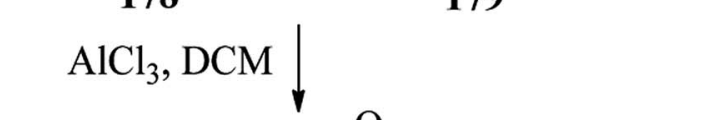<smiles>COc1ccc(-c2ccc(C(=O)CCCCCBr)cc2)cc1</smiles><smiles>CCCCCCCCCCCCCCCCC</smiles><smiles>COc1ccc(-c2ccc(CCCCCCBr)cc2)cc1</smiles>

181<smiles>COc1ccc(/C=C/c2ccc(O)cc2)cc1</smiles><smiles>[Y10]C(=O)OCCCCC(C)=O</smiles>

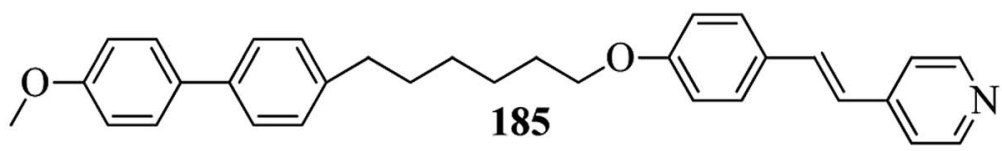

Scheme 22 Synthesis of 185

acetoxystilbazole (120). Afterwards, 4-hydroxystilbazole (121) was synthesized by hydrolyzing the acetic acid ester $\mathbf{1 2 0}$ in ethanolic KOH. In parallel, 6-bromohexyloxymethacrylate (124) was synthesized as described in the previous report by Stumpel et al. ${ }^{56}$ Finally, an Williamson ether synthesis reaction of compounds 121 and 124 utilizing caesium carbonate $\left(\mathrm{Cs}_{2} \mathrm{CO}_{3}\right)$ in anhydrous DMF at room temperature led to the formation of 4'-(6-methacyloxyhexyloxy-4-stilbazole) (125) (Scheme 15). Fig. 10 presents the structure of hydrogen bonded complex 126. The liquid crystal network was prepared by mixing different amounts of cross linker (CL) (trans-4-(4-(11acryloyloxyundecyloxy)cyclohexanecarboxyloxy)phenyl-4-(11acryloyloxyundecyloxy) with 126. To carry out the photopolymerization, $0.5 \mathrm{wt} \%$ thermal inhibitor (butylated hydroxytoluene, BHT) and $1 \mathrm{wt} \%$ photo initiator (Irgacure 819) were added. To obtain a homogeneous mixture, all the compounds were dissolved in dichloromethane solvent. After mixing, the solvent was removed in vacuum. $20 \mu \mathrm{m}$ thick sheets were developed by capillary suction of the liquid crystal-monomer blends in the melt $\left(125{ }^{\circ} \mathrm{C}\right)$ between two exactly separated glass slides (LC cell). After filling the cell, the blend was cooled to $30^{\circ} \mathrm{C}$. Afterwards, the polymerization reaction was performed by UV illumination (provided with a $405 \mathrm{~nm}$ cut-off filter) for about $600 \mathrm{~s}$. To acquire homeotropic and planar alignments, the glass slides (LC cell) were provided with rubbed octadecyltrimethoxysilane and polyimide, respectively. Using POM and DSC, they studied the liquid crystal properties of 126. Smectic A phase was observed at $140{ }^{\circ} \mathrm{C}$ in $1: 1$ aggregates. Smectic $\mathrm{C}$ phase was observed on further cooling from $119{ }^{\circ} \mathrm{C}$ to $110^{\circ} \mathrm{C}$. High levels of tilted smectic phases were noted from $80{ }^{\circ} \mathrm{C}$ to<smiles>CCCCCCCCOc1ccc(C=Cc2cc[nH+]cc2)cc1</smiles><smiles></smiles>

187a-b where $n=4,5$ 
<smiles>NC(=O)c1ccc(-c2cc3sc(-c4ccncc4)nn3n2)cc1</smiles>

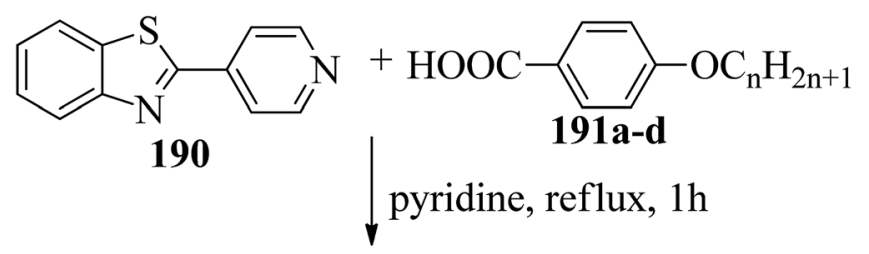

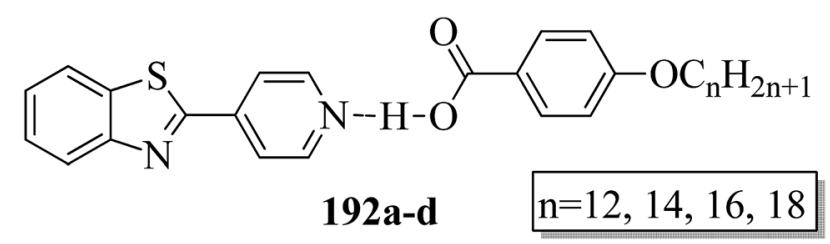

Scheme 24 Preparation of hydrogen bonded complexes 192a-d.

$-4{ }^{\circ} \mathrm{C}$; further lowering the temperature resulted in crystallization. Also, they studied mixtures of 126 with different amounts of cross-linker (CL). The clearing temperature decreases as the amount of $\mathbf{C L}$ increases, and the tilted smectic phase vanishes. For example, when 50 wt $\%$ CL was used, a narrow nematic phase from $119{ }^{\circ} \mathrm{C}$ to $117{ }^{\circ} \mathrm{C}$, a smectic A phase from $117^{\circ} \mathrm{C}$ to $59{ }^{\circ} \mathrm{C}$, and a smectic B phase from $59{ }^{\circ} \mathrm{C}$ to $-15^{\circ} \mathrm{C}$ were observed under POM during the cooling cycle from the isotropic phase. Upon addition of a cross-linker, secured smectic phase can be fixed by photopolymerization. However, the lamellar structure was found to be maintained after removal of the template when $25 \mathrm{wt} \%$ or more cross-linker bearing a nano-porous LCN was used. Also, anisotropic and high anhydrous proton conductivity were obtained by a cationic $2 \mathrm{D}$ nanoporous polymer, which was obtained after immobilization of phosphoric acid $\left(\mathrm{H}_{3} \mathrm{PO}_{4}\right)$ in the LCN pores. Further, these results revealed that $2 \mathrm{D}$ cationic nano-porous polymers can be fabricated with attractive practical properties. It is anticipated that cationic nano-porous polymers which are $\mathrm{pH}$ independent can be fabricated by alkylation of the pyridine component. These polymers are attractive to adsorb and sense or separate negatively charged molecules and anions.

Saccone et al. ${ }^{57}$ prepared fifteen supramolecular complexes using stilbazoles and azophenols as proton acceptors and proton donors, respectively, and studied the effects of ortho-fluorination of the azophenol on the mesogenic behavior. By diazotization and azo-coupling reactions, they synthesized di-fluorinated $(\mathbf{1 2 8 a}-\mathbf{b})$ azophenols. By referring to the report by Bruce et al. ${ }^{\mathbf{5 8}}$ they synthesized the proton acceptors 129a-e. Fig. 11 presents the structures of the hydrogen bond donors (127 and 128a-b) and acceptors 129a-e. All the starting compounds were non-mesogenic in nature. Using POM, they studied the aggregations 127/129ae at a scanning rate of $5{ }^{\circ} \mathrm{C} \mathrm{min}^{-1}$. This study revealed that only the 127/129e complex exhibited liquid crystalline properties (i.e. it exhibited monotropic smectic A phase). Also, different behavior was observed in the ortho-difluorinated azobenzenes 128a-b with stilbazoles (128a/129a-e and $128 \mathrm{~b} / \mathbf{1 2 9 a}-\mathbf{e}$ ) compared with the 127/129a-e complexes. Further, a decreased transition temperature by about $c a$. $50{ }^{\circ} \mathrm{C}$ from the crystalline state to the liquid-crystalline/ isotropic state was observed compared to the $127 / 129 a-e$ complexes. Enantiotropic mesophase was observed in the fluorine-based complexes; they exhibited mesomorphism in a broad range $\left(\sim 50{ }^{\circ} \mathrm{C}\right)$ during the heating cycle, whereas during the cooling cycle, they exhibited mesomorphism in an even broader range $\left(\sim 80^{\circ} \mathrm{C}\right)$. As the alkyl chain length at the stilbazoles increased in the 128a- and 128b-based complexes, their transition temperatures from the liquid crystal state to the isotropic state also increased, except in the $128 a / 129 c$ and $128 b / 129 e$ complexes, where minute decreases in the clearing temperatures were noted compared to the complexes 128a/129a and 128b/129d, respectively. For the short-alkyl-chain complexes (129a and 129b), nematic phase was spotted; at the same time, smectic A phase was predominantly observed for the long-alkylchain complexes (129d and 129e). A dissimilarity was observed for the 129c complexes, which exhibited nematic phase when complexed with 128a and exhibited smectic phase when complexed with 128b. The typical texture of smectic E phase was noted in the $128 \mathrm{a} / 129 \mathrm{c}, 128 \mathrm{~b} / \mathbf{1 2 9 c}$ and 128b/129e complexes. Hence, this distinctly organized mesophase is structurally related to the smectic A phase; however, with the maintained orthorhombic lattice, it generally arises between the crystalline phase and smectic A 
View Article Online

RSC Advances

Review

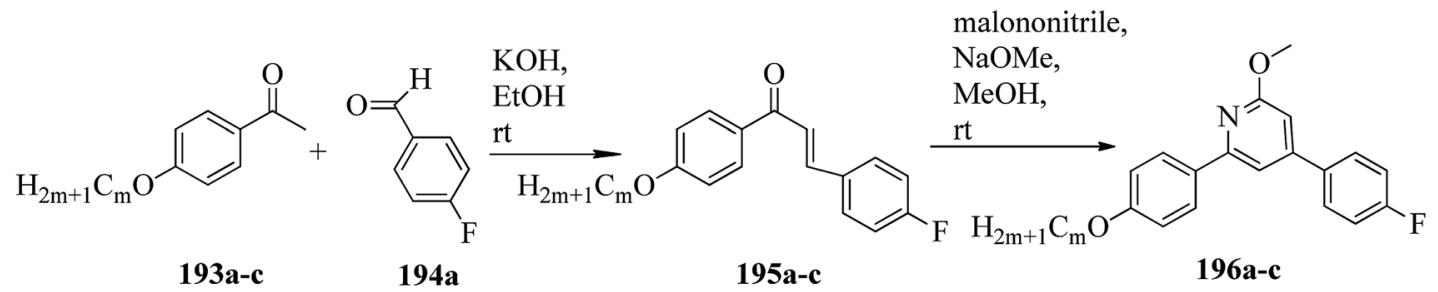

Where $\mathrm{m}=10,12,14$

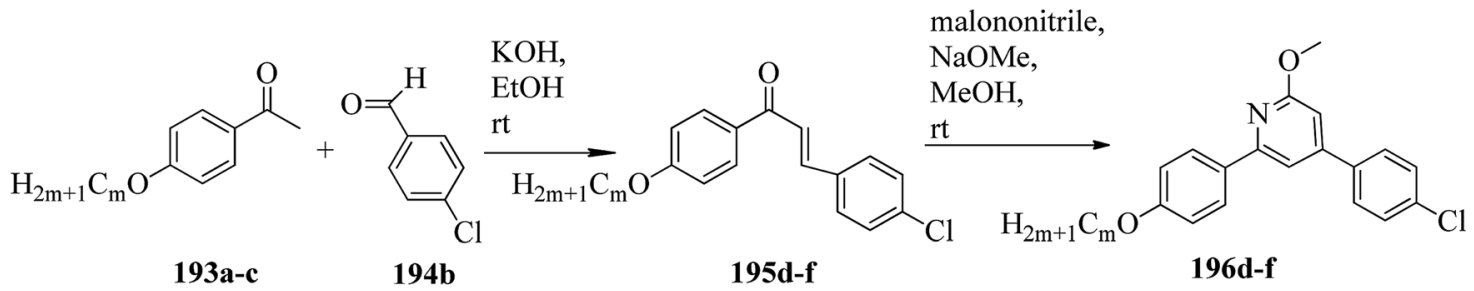

Where $\mathrm{m}=10,12,14$<smiles>COc1cc(-c2ccc(Br)cc2)cc(-c2ccc(OCCOc3ccc(C(=O)C=Cc4ccc(Br)cc4)cc3)cc2)n1</smiles>

Where $\mathrm{m}=10,12,14$

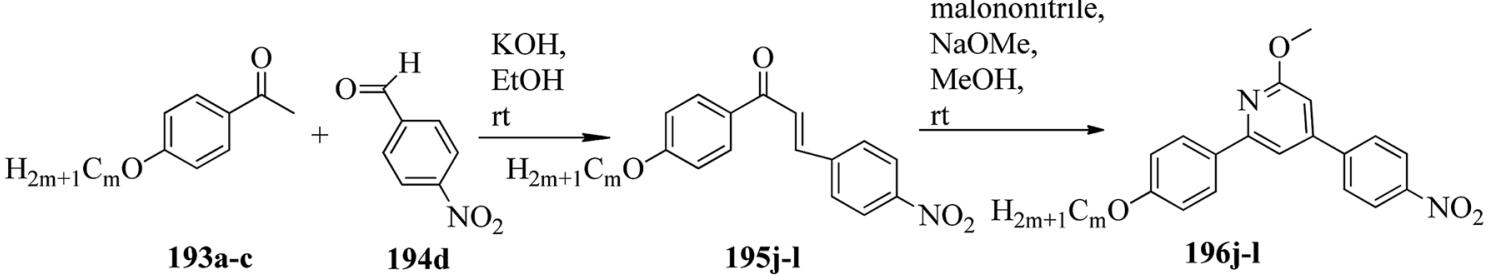

Where $\mathrm{m}=10,12,14$

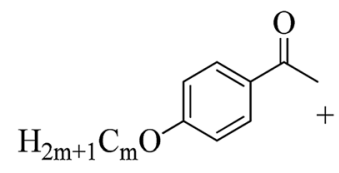<smiles>O=Cc1ccncc1</smiles><smiles>[14CH3]Oc1ccc(C(=O)/C=C/c2ccncc2)cc1</smiles><smiles>COc1cc(-c2ccncc2)cc(-c2ccc(OC)c(OC)c2)n1</smiles>

$193 c$

$194 \mathrm{e}$

$195 \mathrm{~m}$

$196 \mathrm{~m}$

Where $\mathrm{m}=14$

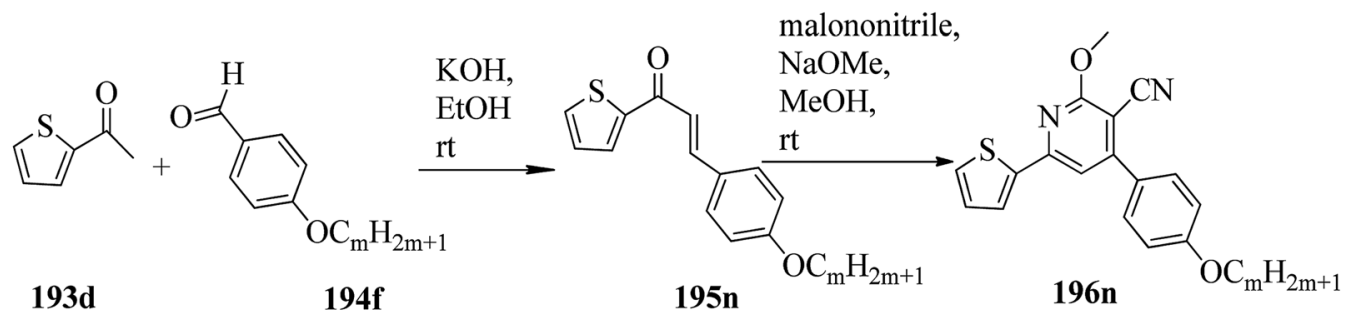

Where $m=10$

Scheme 25 Synthetic route for the fourteen blue luminescent mesogens $196 a-n$.

phase. Further, they studied the photo-induced phase transition in the prepared complexes by illuminating with $405 \mathrm{~nm}$ LED and viewing in situ through POM. Upon illumination, the mesogenic alignment quickly vanished at $90{ }^{\circ} \mathrm{C}$ because of the photo-induced phase transition to isotropic phase. This phase transition was due to the trans-

23186

| RSC Adv., 2019, 9, 23161-23228

This journal is (C) The Royal Society of Chemistry 2019 
<smiles>O=Cc1ccc(O)c(O)c1</smiles>

197<smiles>CC(=O)c1ccc(O)cc1</smiles>

199<smiles>O=Cc1ccc(O)cc1</smiles>

200<smiles>CCCCCCOc1ccc(C=O)cc1OCC</smiles>

198a-e ammonium acetate, 1,4-dioxane, reflux, $24 \mathrm{~h}$

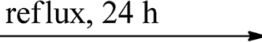<smiles>N#Cc1c(-c2ccc(O)cc2)cc(-c2ccc(O)cc2)[nH]c1=O</smiles>
ethyl chloroacetate, anhydrous $\mathrm{K}_{2} \mathrm{CO}_{3}$, DMF, $80^{\circ} \mathrm{C}, 12 \mathrm{~h}$<smiles>N#Cc1c(-c2ccc(OCC(=O)NN)cc2)cc(-c2ccc(OCC(=O)NN)cc2)nc1OCC(=O)NN</smiles>

Where $\mathrm{m}=8,10,12,14,16$.

201<smiles>CCOC=O</smiles> 
<smiles></smiles>

205a-f

206

.

even at $60{ }^{\circ} \mathrm{C}$ and $30{ }^{\circ} \mathrm{C}$. Hence, these complexes can be utilized in photonics.

Wang et al..$^{59}$ prepared two series of ionic liquid crystals from diphenylviologens. Further, they synthesized the precursors diphenyl viologen dichloride $\mathbf{1 3 3}$ and di(4-alkoxyphenyl) viologen dichlorides 134a-c $\left(\mathrm{R}=\mathrm{C}_{n} \mathrm{H}_{2 n+1}, n=10,12,14\right)$ through Zincke reactions by referring to previous literature reports by Nanasawa et al. ${ }^{60}$ and Kuo et $a l^{48}$ During the metathesis of counter anions, methanol was used as the phase transfer atmosphere. The counter anion salts such as sodium tetrafluoroborate $\left(\mathrm{NaBF}_{4}\right)$, lithium triflate (LiOTf), potassium thiocyanate $(\mathrm{KSCN})$, sodium alkylsulfonate $\left(\mathrm{RSO}_{3} \mathrm{Na}\right)$, potassium hexafluorophosphate $\left(\mathrm{KPF}_{6}\right)$, and lithium bis(trifluoromethane)sulfonimide $\left(\operatorname{LiNTf}_{2}\right)$ were dissolved in water and added to a solution of methanol/dichloromethane $\left(\mathrm{CH}_{3} \mathrm{OH} /\right.$ $\left.\mathrm{CH}_{2} \mathrm{Cl}_{2}=1 / 5\right)$ containing the precursors 133 or 134a-c. The reaction mixture was stirred for $3 \mathrm{~h}$ at $35^{\circ} \mathrm{C}$ to $40^{\circ} \mathrm{C}$, and the reactions were monitored using alumina TLC plates with $\mathrm{CH}_{3} \mathrm{OH} / \mathrm{CH}_{2} \mathrm{Cl}_{2}$ (5/95) eluent. Finally, the crude product was passed through an alumina $\left(\mathrm{Al}_{2} \mathrm{O}_{3}\right)$ flash column, followed by recrystallization using an isopropanol/ethyl acetate mixture to afford the pure product (Scheme 16). The crystallographic data of two single crystals of $\mathbf{1 3 5 a}$ and $\mathbf{1 3 6} \mathrm{b}$ revealed that the presence of intermolecular hydrogen bonds induced the mono- or bilayer-lamellar structure. All the synthesized ionic liquids exhibited mesogenic behavior. Smectic A phase with a homeotropic texture or focal conic texture was generally observed during the cooling cycle. Powder XRD studies also revealed that smectic A phases exist as monolayer structures for 136-141 or bilayer structures for 135a-c. Further, the melting temperatures $\left(T_{\mathrm{mp}}\right)$ remained at $T_{\mathrm{mp}}=88.7^{\circ} \mathrm{C}$ to $113.1{ }^{\circ} \mathrm{C}$ in the viologen 135a-c series; however, the clearing temperature $\left(T_{\mathrm{cl}}\right)$ increased with the chain length, i.e. $T_{\mathrm{cl}}=217^{\circ} \mathrm{C}(\mathbf{1 3 5 a})<256{ }^{\circ} \mathrm{C}(\mathbf{1 3 5 b})<$ $275{ }^{\circ} \mathrm{C}(\mathbf{1 3 5 c})$. The extent of smectic A phase increased with the chain length during the cooling cycle: $\Delta T_{\mathrm{SmA}}=118.5^{\circ} \mathrm{C}(\mathbf{1 3 5 a})<$ $196.3^{\circ} \mathrm{C}(\mathbf{1 3 5 b})<223.0^{\circ} \mathrm{C}(\mathbf{1 3 5 c})$. Smectic A phase was observed in viologen derivatives 136-141. However, the clearing point was found to be quite sensitive to the incorporated anions; $T_{\mathrm{cl}}=$ $337.0{ }^{\circ} \mathrm{C}(\mathbf{1 3 8 b})>336.0{ }^{\circ} \mathrm{C}(\mathbf{1 3 6 b})>312.0{ }^{\circ} \mathrm{C}(\mathbf{1 3 7 b})>304.8{ }^{\circ} \mathrm{C}$ $(139 b)>274.0{ }^{\circ} \mathrm{C}(141 b)>220.2{ }^{\circ} \mathrm{C}(140 b)$ for the $n=12$ derivative. Substituted alkyl-chains at the anion and cation parts of compounds 140a-c displayed higher melting temperatures $\Delta T_{\mathrm{mp}}=31.7^{\circ} \mathrm{C}(\mathbf{1 4 0 a})<43.8^{\circ} \mathrm{C}(\mathbf{1 4 0 b})<50.0^{\circ} \mathrm{C}(\mathbf{1 4 0 c})$ and lower clearing temperatures $\Delta T_{\mathrm{cl}}=0.3^{\circ} \mathrm{C}(\mathbf{1 4 0 a})<35.8^{\circ} \mathrm{C}$ $(140 b)<71.3{ }^{\circ} \mathrm{C}(140 \mathrm{c})$ than compounds $135 \mathrm{a}-\mathrm{c}$. This alteration in both the melting and clearing temperatures results in a smaller smectic A phase range, i.e. $\Delta T_{\mathrm{SmA}}=92.4{ }^{\circ} \mathrm{C}(\mathbf{1 4 0 a})$ to $86.0^{\circ} \mathrm{C}(\mathbf{1 4 0 c})$; this can be ascribed to the greater number of hydrophobic interactions than of hydrogen bond interactions and coulombic interactions. Similarly, in the viologen series 139a-c, the melting temperatures remained at $103.8{ }^{\circ} \mathrm{C}$ to $104.5^{\circ} \mathrm{C}$, but the clearing temperatures increased with carbon chain length, i.e., $T_{\mathrm{cl}}=254.3{ }^{\circ} \mathrm{C}(139 a)<304.8{ }^{\circ} \mathrm{C}(139 \mathrm{~b})<$ $328.0{ }^{\circ} \mathrm{C}(\mathbf{1 3 9 c})$. The smectic A phase range increased with the carbon chain length during the cooling cycle: $\Delta T_{\mathrm{SmA}}=156.3^{\circ} \mathrm{C}$ (139a) $<203.3{ }^{\circ} \mathrm{C}(\mathbf{1 3 9 b})<228.8^{\circ} \mathrm{C}(\mathbf{1 3 9 c})$. They concluded that elongation of the alkyl chain length increases the clearing and melting temperatures. In order to comprehend the effects of the counter anions on the mesogenic properties in diviologens, they prepared six compounds with different anions $\left(\mathrm{X}=\mathrm{BF}_{4}, \mathrm{PF}_{6}\right.$, OTf, $\left.\mathrm{NTf}_{2}, \mathrm{RSO}_{3}, \mathrm{SCN}\right)$. All the prepared compounds exhibited mesogenic properties. They compared these bipyridinium- 


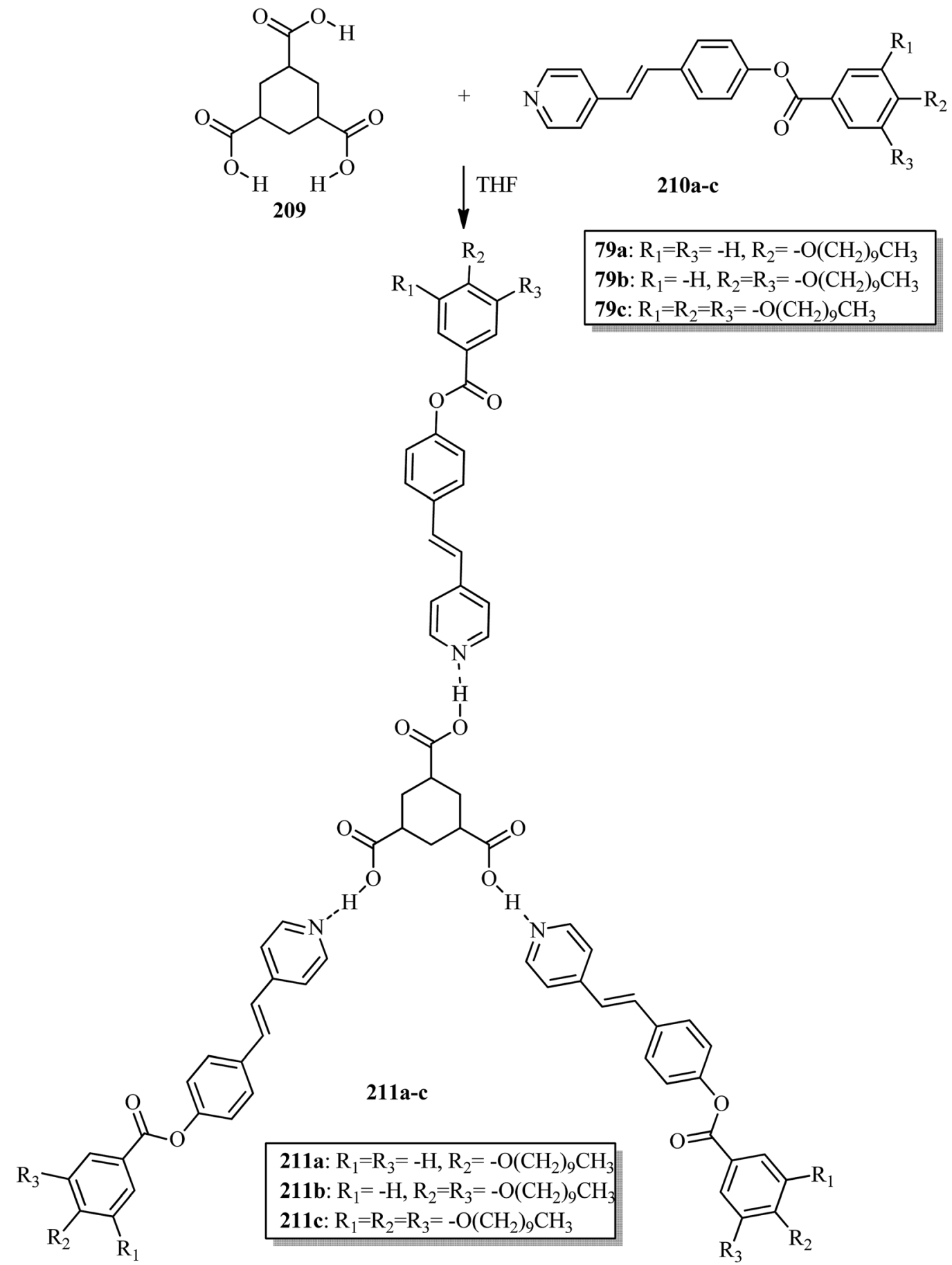

Scheme 28 Synthesis of hydrogen bonded complexes $211 a-c$.

based mesogens with pyridinium-based mesogens (literature from Wang et al. ${ }^{44}$ ) and found that the bipyridinium-based mesogens exhibited wider $\Delta T_{\mathrm{SmA}}$ ranges and higher $T_{\mathrm{cl}}$ values than the pyridinium-based mesogens. Decomposition before the clearing point was observed in the ionic salts with small anionic moieties, i.e. $\mathrm{X}=\mathrm{BF}_{4}, \mathrm{PF}_{6}$, OTf, SCN. In compounds 136b and 141b, they noted more ordered smectic $X$ phase, which was also confirmed using powder XRD analysis.
Bhat et al. ${ }^{61}$ prepared two series of hydrogen bonded liquid crystalline materials by mixing non-mesomorphic fatty acids such as nonanoic (145a), capric (145b), undecanoic (145c), tridecanoic (145d), myristic (145e), palmitic (145f) and stearic (145g) acids with (4-pyridyl)-benzylidene- $p$ - $n$-alkylanilines (144a-b; $n=12$ or 16). Condensation of 4-pyridine carboxaldehyde and the corresponding anilines, such as $4-n$-dodecylaniline (143a) and 4- $n$-hexadecylaniline (143b), in the presence of a catalytic amount of glacial acetic acid yielded (4-pyridyl)- 
<smiles>CON=CON(C)OC</smiles>

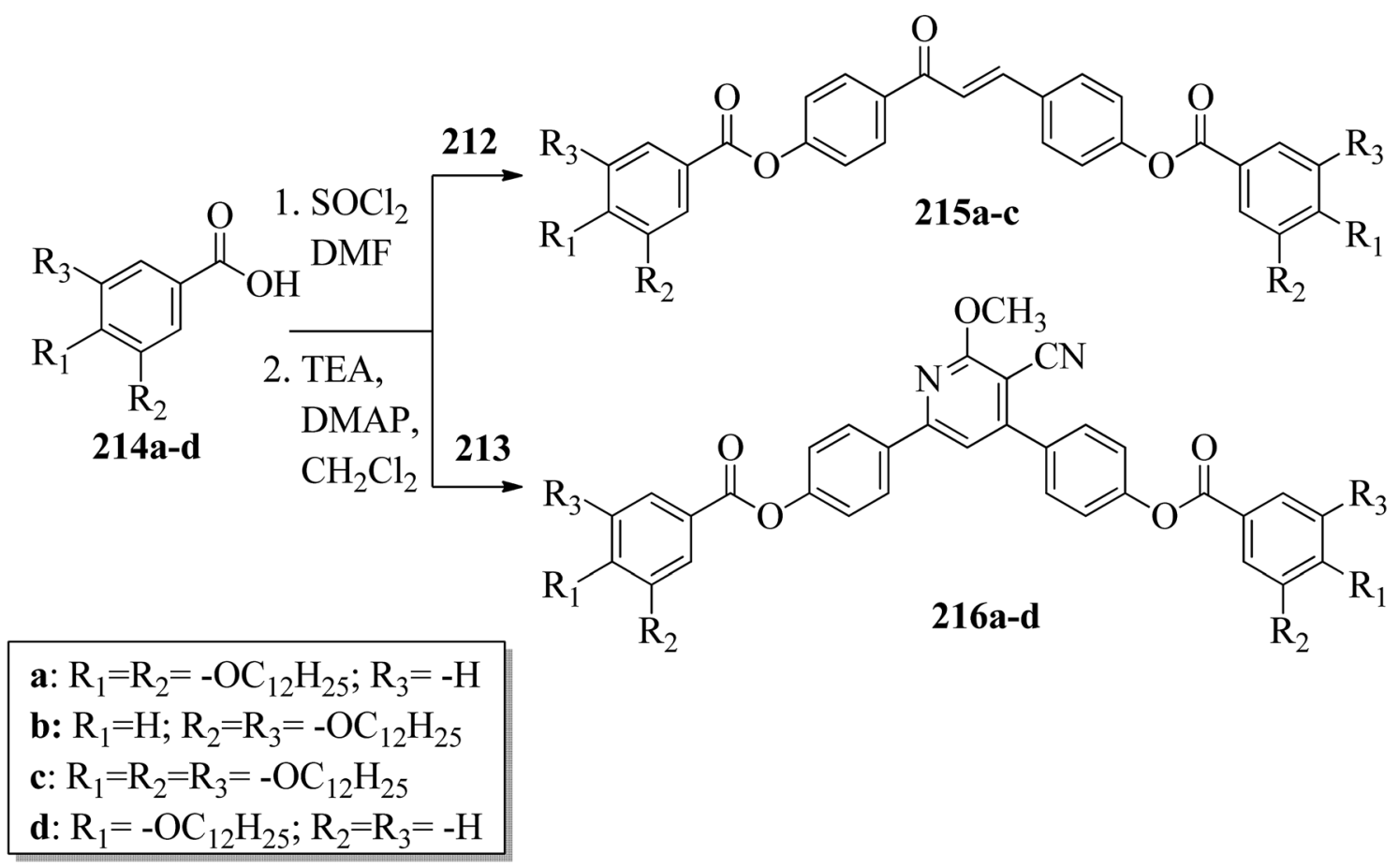

Scheme 29 Synthetic route for compounds $215 a-c$ and $216 a-d$.

benzylidene-4'- $n$-alkylanilines 144a-b. The obtained products were recrystallized using ethanol solvent. The proton donors, i.e. fatty acids, and proton acceptors were dissolved separately in THF. The two solutions were mixed, refluxed for $1 \mathrm{~h}$ and stirred at room temperature overnight. Finally, the solvent was removed by distillation to form the expected hydrogen bonded complexes (Scheme 17). Using FT-IR spectroscopy, they confirmed the formation of hydrogen bonds between the fatty acids (145a-g) and (4-pyridyl)-benzylidene- 4 '- $n$-alkylanilines $(\mathbf{1 4 4 a}-\mathbf{b})$. All the starting materials were non-mesogenic in nature; however, the prepared complexes exhibited monotropic smectic B phases. Further, they observed that the DSC thermograms of the $\mathbf{1 4 4 b / 1 4 5 a}$ and $\mathbf{1 4 4 b} / \mathbf{1 4 5 d}$ aggregations were folded in the direction of the high temperature side. This abnormal characteristic may arise because of the enormous amount of heat released, during which the sample pan temperature was higher than that of the reference pan. It was observed that fatty acid complexes with odd numbers of carbon atoms showed lower mesomorphic thermal ranges than the complexes of fatty acids with even numbers of carbon atoms. The higher thermal ranges of the mesogenic phases in complexes with even-numbered carbon atoms were ascribed to the strong intermolecular interactions which were caused by the large surface areas of the long chains as well as to the beneficial longitudinal dipole moments of the molecules along the long molecular axes of the hydrogen bonded complexes. The thermal ranges of the mesogens of the $\mathbf{1 4 4 b} / \mathbf{1 4 5 a}-\mathrm{g}$ series were found to be slightly lower than those of the 144a/145a-g series. This is because of the long chain length of the proton acceptor, where the projection of the end chain dilutes the core of the molecule and causes disorder in the orientation. In both series, they observed that the clearing temperatures increased as the number of carbon atoms in the fatty acids increased. This was attributed to the increasing molecular weight of the resulting hydrogen bonded complexes. They also compared the mesogenic properties with their previous report (Bhat et al. ${ }^{62}$ ) and concluded that the $\pi \cdots \mathrm{C}-\mathrm{H}$ interactions and $\pi-\pi$ stacking interactions play crucial roles while exhibiting mesomorphic properties. Smaller thermal ranges of mesomorphic phase than those of the pure alkoxy benzoic acid dimeric mesogens were observed in the 144a-b/145a-g complexes with two aromatic rings.

Ong et al. ${ }^{63}$ synthesized calamitic mesogens, $4-\{[($ pyridin-4-yl) methylidene]amino\}phenyl 4-alkoxybenzoates (152a-i), containing pyridine heterocycles and a two-phenyl core ring system, imine linkage, ester linkage, and alkoxy chain (which was varied 


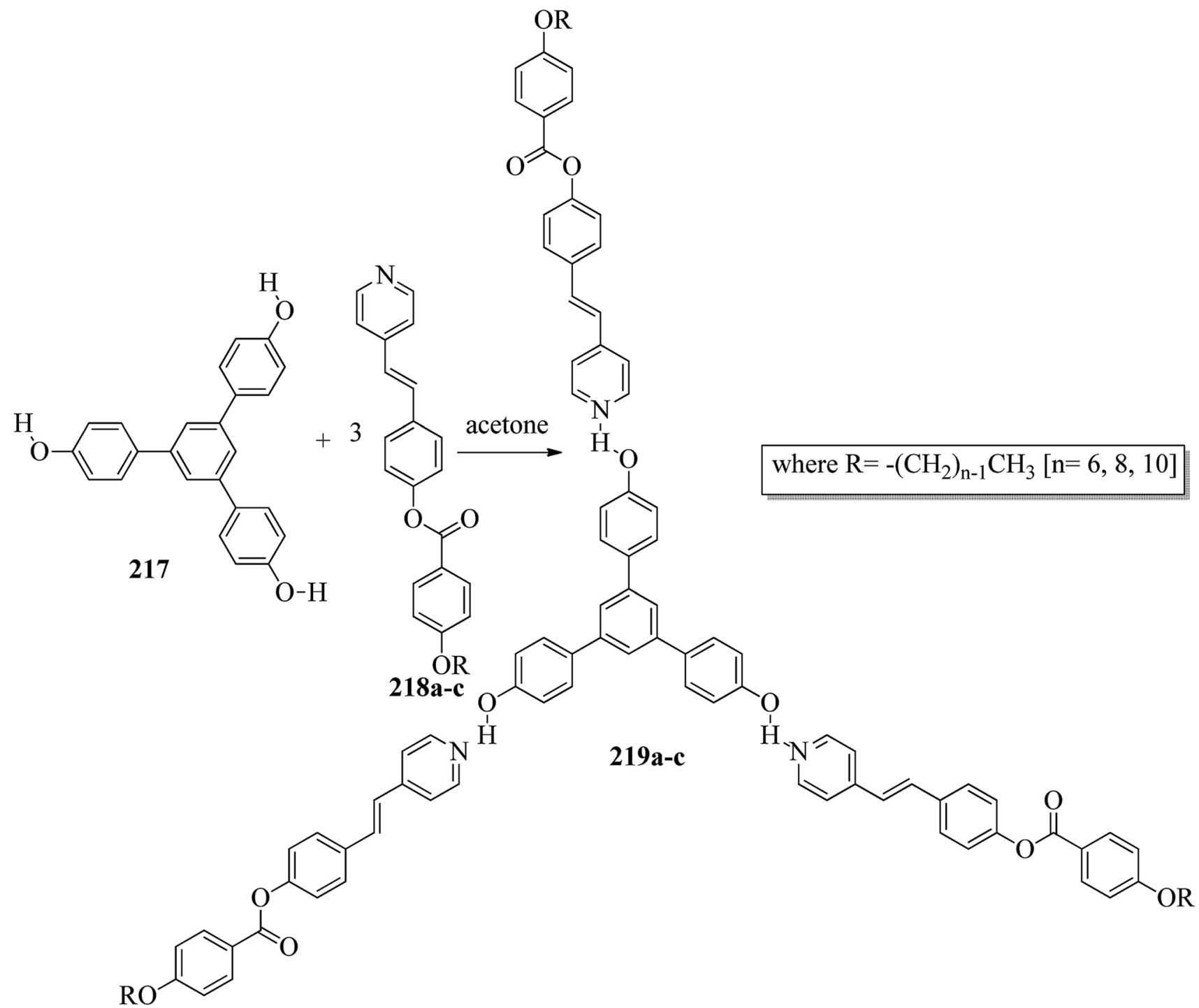

Scheme 30 Preparation of supramolecular discotic liquid crystals (219a-c).

as $n=2,4,6,8,10,12,14,16,18)$. Further, they referred to previous reports by $\mathrm{Ha}$ et al. ${ }^{64}$ and Kadkin et al. ${ }^{65}$ to synthesize the compounds 4-[(pyridine-4-ylmethylene)amino]phenol (148) and 4-alkoxybenzoic acid (151a-i), respectively. Compounds 148, 151a-i, and DMAP were dissolved in a mixture of DMF and DCM solvents, and the mixture was stirred at $0{ }^{\circ} \mathrm{C}$. Further, the required amount of DCC was dissolved in DCM and added dropwise to the reaction mixture, which was then stirred at $0{ }^{\circ} \mathrm{C}$ for an hour. The mixture was later stirred for $3 \mathrm{~h}$ at room temperature. Finally, the reaction mixture was filtered and the solvent was evaporated to obtain the expected products (Scheme 18). The first three compounds, i.e. 152a-c $(n=2,4$ and 6$)$ in the series exhibited nematic mesophase. In addition to nematic phase, smectic A phase was also observed as the alkoxy chain length increased to $n=8$ and $n=10$. However, when the alkoxy chain length moved from $n=12$ to $n=18$, the nematic phase vanished and only smectic A phase was observed in these complexes. Ester linkages provide greater dipole-dipole interactions, which influences the lateral packing; due to this, smectic phase was generated. Hence, additional smectic A phase was observed along with nematic phase as the carbon chain length increased to C-8. The melting temperatures decreased as the chain length varied from C-2 to C-12 and further increased with increasing chain length from C-12 to C18. However, the clearing temperature decreased throughout the series because long carbon chains cause dilution of the mesogenic core. The smectic phase range increased as the chain length increased from $\mathrm{C}-8$ and reached the highest mesophase range with $\mathrm{C}-12$; thereafter, the smectic A phase range decreased as the chain length increased from C-14 to C-18. Hence, the widest smectic A phase range was observed in $\mathbf{1 5 2 f}$ $\left(\Delta \mathrm{SmA}=51.3^{\circ} \mathrm{C}\right)$ and the widest nematic phase was observed in 152a $\left(\Delta N=53.0^{\circ} \mathrm{C}\right)$. They also compared these compounds with previously reported compounds, i.e. 153 (Ha et al. ${ }^{64}$ ), 154 (Lim et al. ${ }^{66}$ ) and 155 (Sakurai et al. ${ }^{67}$ ). The structures of 153, 154, and 155 are presented in Fig. 22. Compound 153 with a pyridine ring 
<smiles>O=C(O)c1cc(C(=O)O)cc(C(=O)O)c1</smiles><smiles>Oc1nc(O)nc(O)n1</smiles><smiles>Oc1cc(O)cc(O)c1</smiles>

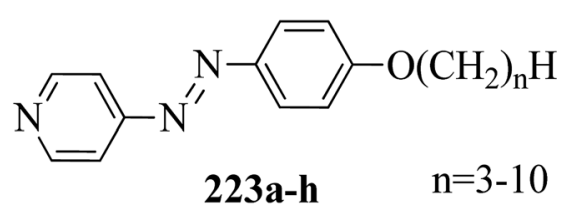

Fig. 13 Structures of proton donors 220, 221, and 222 and the proton acceptors 223a-h.

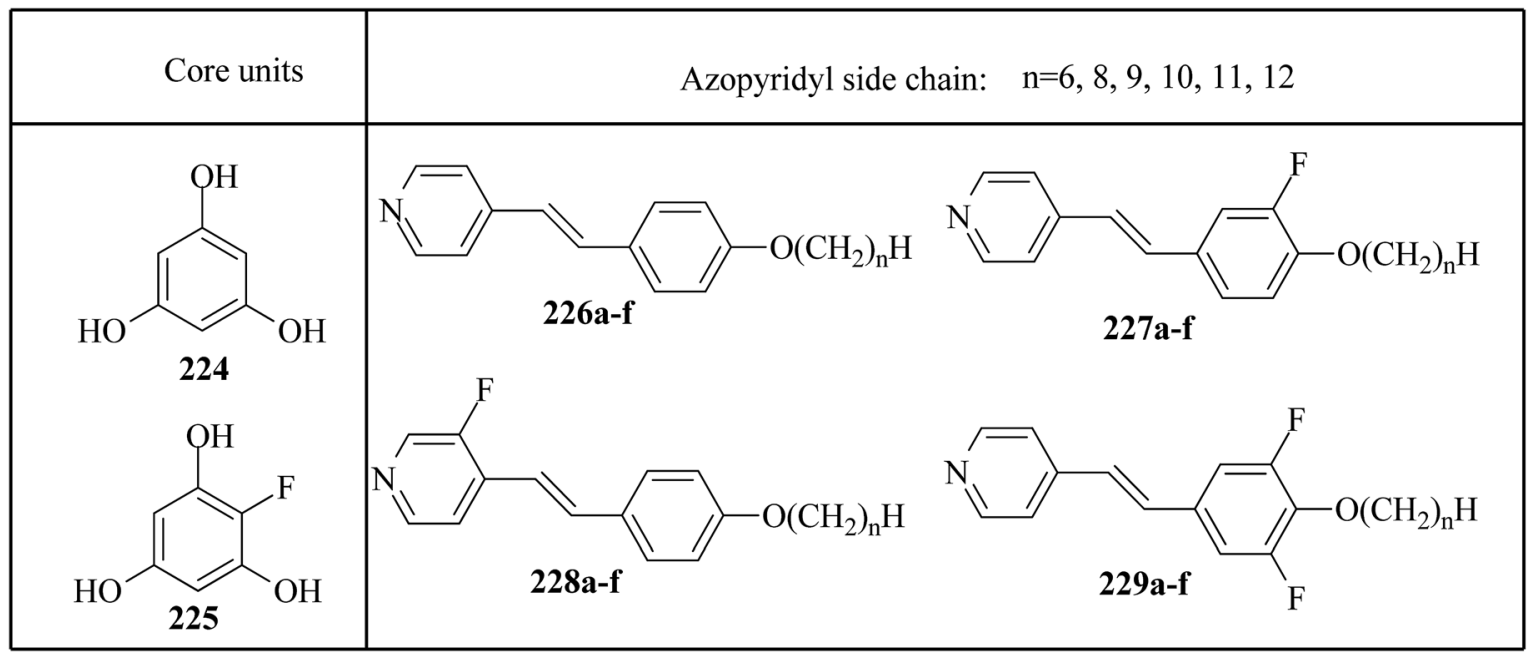

Fig. 14 Structures of the core units and azopyridyl side chains.

and aromatic ring did not display mesogenic properties although the two rigid rings were connected by a Schiff base group. When another rigid aromatic ring was added to this compound, it became $\mathbf{1 5 2} \mathbf{g}$ (the title compound). The addition of this rigid aromatic ring increases the length of the molecule and does not change the width of the compound. Hence, they concluded that for the generation of mesophases, this longer molecule was more suitable. Also, the stability of the phases was found to be higher for the three-ring molecule (152g) than for the two-ring molecule (compound 153). Next, they compared $\mathbf{1 5 2 g}$ with the three-ring system 154; the connecting unit between the aromatic ring and pyridine ring was the only difference between these two compounds. Compound 154 possesses an enone as the linking unit, whereas a Schiff base is the linking group in 152g. A lower melting point of about $99.2^{\circ} \mathrm{C}$ and a wider smectic A phase range of about $\Delta \mathrm{SmA}=$

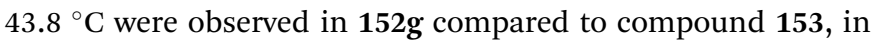
which a melting point of about $109.8^{\circ} \mathrm{C}$ and a smectic A phase range of about $\Delta \mathrm{SmA}=7.3{ }^{\circ} \mathrm{C}$ were observed. The molecular linearity of the title molecule was maintained, although it has a stepped core structure due to the Schiff base linkage; therefore, the compound induces mesophase formation and provides better stability. Hence, they concluded that the Schiff base connecting group performs a notable role in minimizing the melting temperature and then broadens the range of the mesophases. Compound 154 also contains three aromatic rings connected by ester linkages. This aromatic-ester compound exhibited a smectic A phase range of about $20{ }^{\circ} \mathrm{C}$ from the starting temperature of $113{ }^{\circ} \mathrm{C}$. When the aromatic-ester complex was changed to a pyridine-Schiff base complex, i.e. molecule 152g, its melting temperature decreased; accordingly, this broadened the mesophase range. Hence, they concluded that the superior mesogenic properties in $152 \mathrm{~g}$ were contributed by the pyridine nucleus complex and Schiff base linking unit (Fig. 12).

Du et al. ${ }^{68}$ prepared halogen bonded complexes using 1,2dibromotetrafluorobenzene (158) or 1,2-diiodotetrafluorobenzene (157) and azopyridine derivatives (156). Halogen bond acceptors, i.e. azopyridine derivatives (156), were synthesized by diazotization and azo coupling reactions. The authors used commercially available halogen bond donors 157 and 158 . Further, they prepared supramolecular complexes by mixing equimolar ratios of halogen-bonded acceptor 156 and halogenbonded donors 157 or 158 in $n$-hexane and petroleum ether 


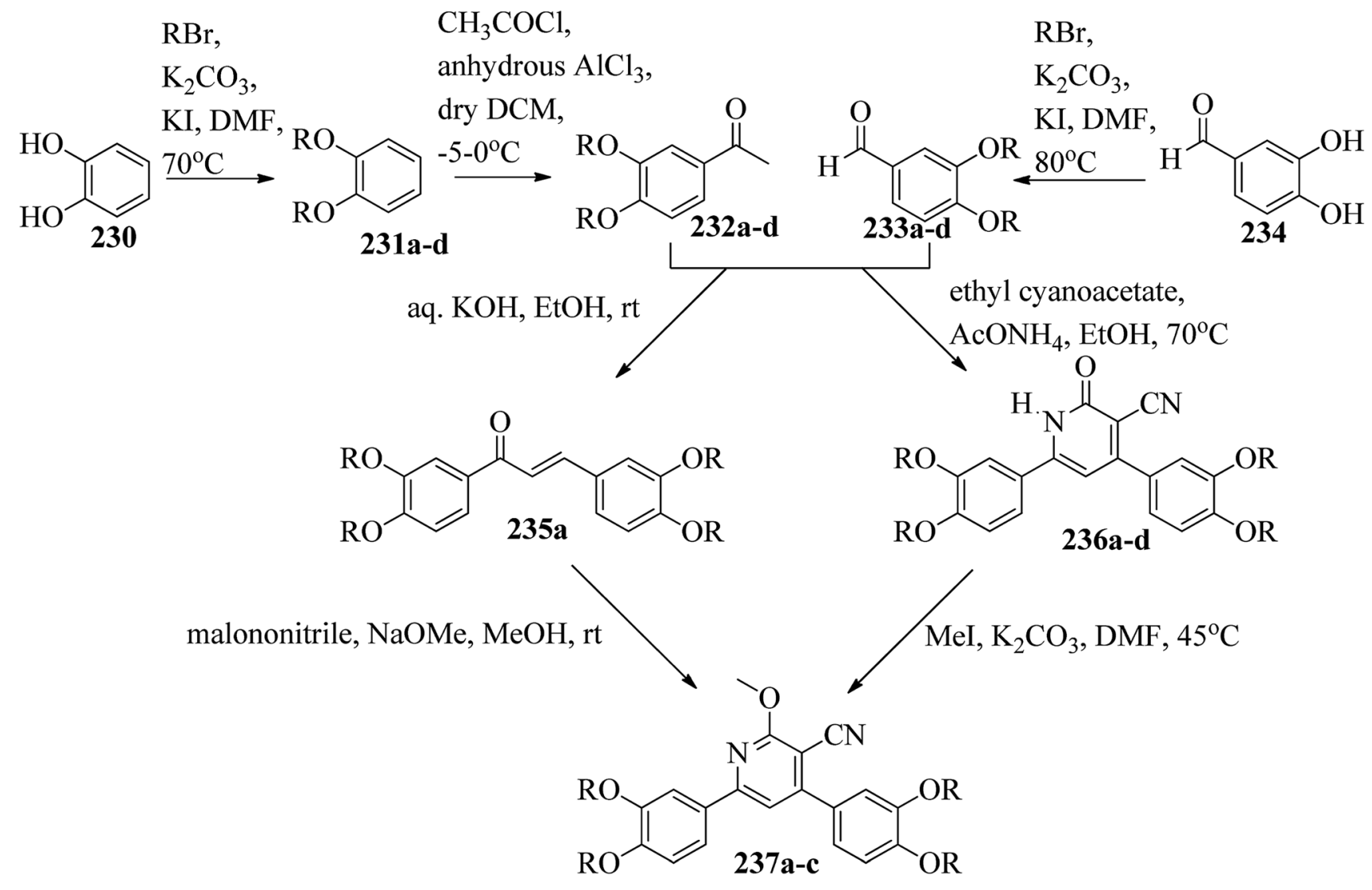

$\mathrm{R}=\mathrm{n}-\mathrm{C}_{6} \mathrm{H}_{13}$, for 231a, 232a, 233a, 235a, 236a, 237a

$\mathrm{R}=\mathrm{n}-\mathrm{C}_{8} \mathrm{H}_{17}$, for 231b, 232b, 233b, 236b, 237b

$\mathrm{R}=\mathrm{n}-\mathrm{C}_{12} \mathrm{H}_{25}$, for 231c, 232c, 233c, 236c, 237c

$\mathrm{R}=\mathrm{n}-\mathrm{C}_{16} \mathrm{H}_{33}$, for 231d, 232d, 233d, 237d

Scheme 31 Synthesis of 237a-c.<smiles>[Y19][14CH2]c1ccc(N=Nc2ccc(C(=O)O)cc2)cc1</smiles>

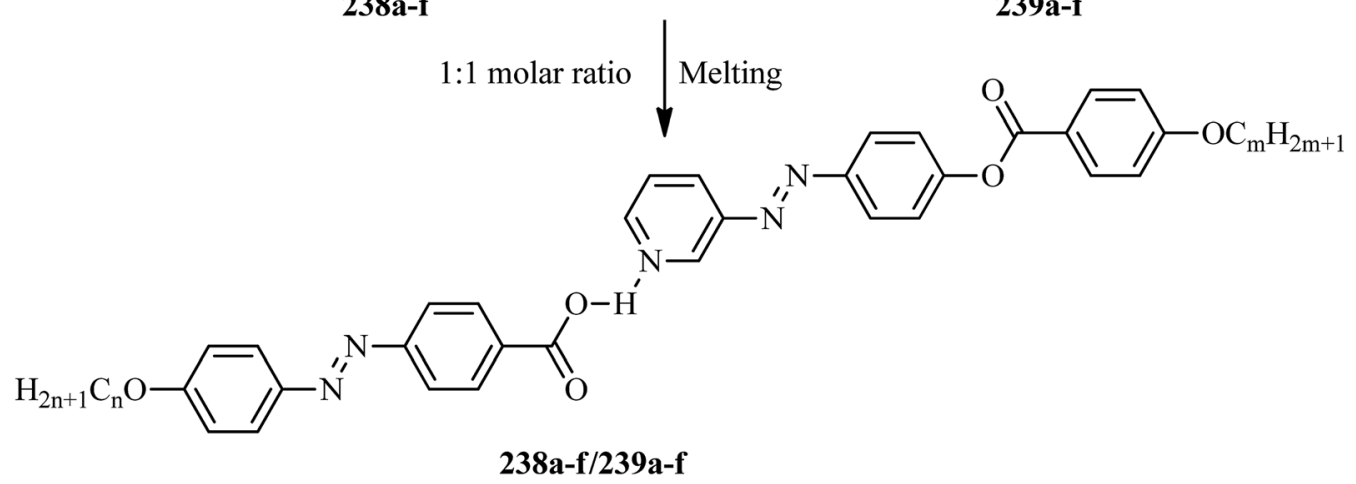

Scheme 32 Preparation of supramolecular complexes $238 a-f / 239 a-f$. 


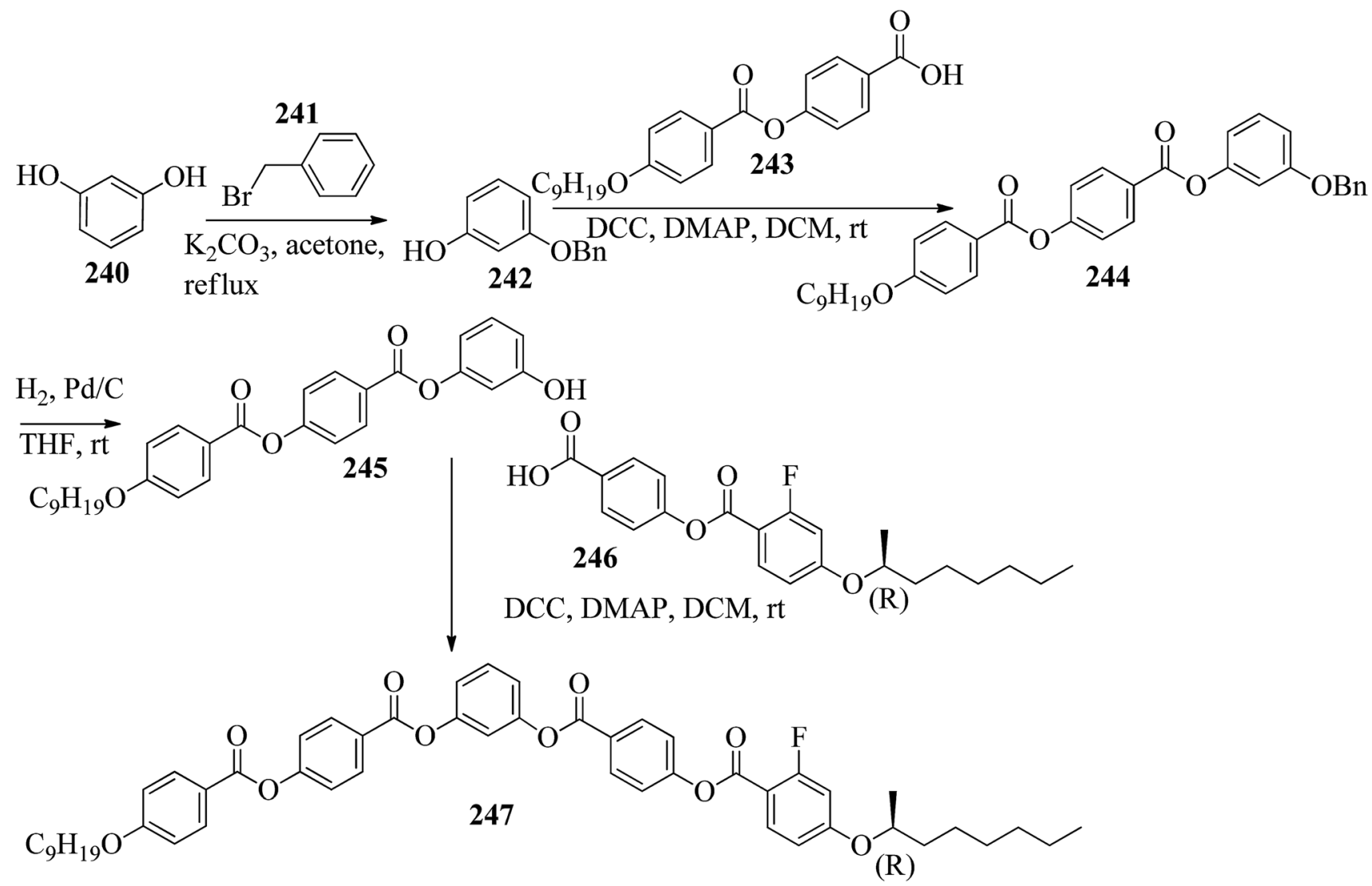

Scheme 33 Synthetic route for the covalent bent core 247.

mixed solvent. The mixture was stirred for 7 to $8 \mathrm{~h}$ at room temperature. Finally, the expected complexes were obtained by evaporating the solvents (Scheme 19). Monotropic mesophases were observed in the iodine-bonded complexes 159c-h $(n=10$ to 15), i.e. on heating, the complexes directly melted to the isotropic state from the crystalline state; however, during the cooling cycle they exhibited the focal conic fan texture of smectic A phase in the temperature range of about $45{ }^{\circ} \mathrm{C}$ to $30{ }^{\circ} \mathrm{C}$. Further, they prepared the bromine-bonded complexes for comparison with the iodine-bonded complexes. Unfortunately, the bromine-bonded complexes did not exhibit any significant mesogenic character; this may be because of the weak $\mathrm{N} \cdots \mathrm{Br}$ interactions in the liquid state, which is similar to a previous literature report of halogen-bonded aggregates of 1,4dibromotetrafluorobenzene and alkoxystilbazoles (González et $\left.a l .{ }^{69}\right)$. To study the photo-induced phase transitions in the prepared complexes, further, they illuminated them with UV light of $365 \mathrm{~nm}\left(80 \mathrm{~mW} \mathrm{~cm}^{-2}\right)$. Upon illumination at $41^{\circ} \mathrm{C}$, the birefringent smectic A phase vanished after $3 \mathrm{~s}$ because of the photo-induced phase transition to the isotropic phase. This phase transition is due to the trans-cis isomerization of the azopyridine group, and the mesophase destabilization was due to the bent structures of the cis-azopyridine groups. When the irradiation was halted, mesogenic textures emerged.

Alaasar and Tschierske ${ }^{70}$ prepared hydrogen bonded supramolecular mesogenic complexes using 4-(4'-pyridylazophenyl)- $4^{\prime}$-alkoxybenzoates (162a-d) as proton acceptors and 4-[5-(4'cyanobiphenyl-4-yloxy)alkyloxy]benzoic acids (163a-b) as proton donors. According to the synthetic route given in a previous report by Naoum et al.,$^{71}$ they synthesized the pyridine-based components (162a-d) by esterification reactions between 4- $n$-alkoxybenzoic acids and 4-(4'-pyridylazo)-phenol (161). By referring to previous publications, i.e. reports by Alaasar and Tschierske, ${ }^{72}$ they synthesized the proton acceptor compounds $(\mathbf{1 6 3 a}-\mathbf{b})$. The non-reported compound 4-(4'-pyridylazophenyl)-4'-octyloxybenzoate (162a) was synthesized by stirring the compounds 161, octyloxybenzoic acid, DCC, and DMAP catalyst in a mixture of $\mathrm{CH}_{2} \mathrm{Cl}_{2}$ and THF $(0.75: 0.25)$ for $48 \mathrm{~h}$ at room temperature. The obtained solid was filtered and washed with $\mathrm{CH}_{2} \mathrm{Cl}_{2}$. After removal of the solvents under vacuum, the product was purified by column chromatography using $\mathrm{CH}_{2} \mathrm{Cl}_{2}$ as the eluent. The expected complexes were prepared by melting 162a-d and 163a-b in an equimolar ratio with stirring. The solid obtained after cooling was ground and the process was repeated until a homogeneous mixture formed (Scheme 20). Initially, they studied the mesogenic properties of the pure parent compounds; this study revealed that the transition temperatures of compound 163a are in good accord with the same compounds previously reported by Jansze et $a l .{ }^{73}$ They observed slightly different transition temperatures of the pyridine-based components (162b-d) than the same compounds which were previously reported by Naoum et al. $;^{71}$ 


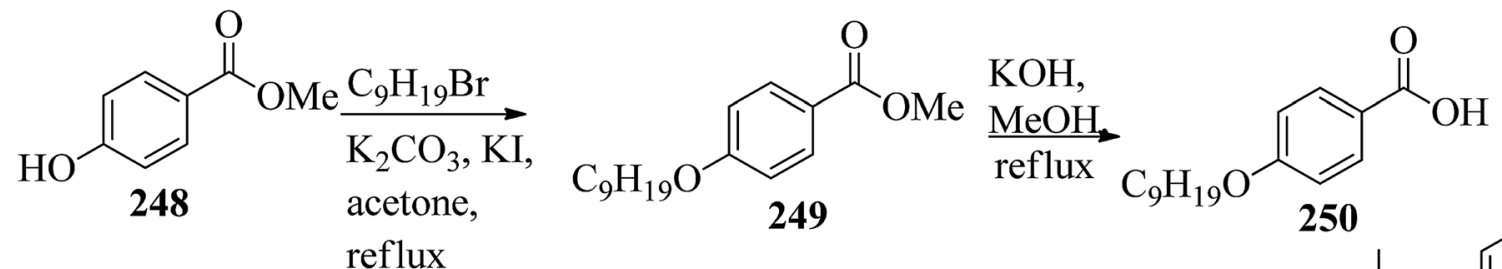
reflux

249 DCC, DMAP, DCM, rt

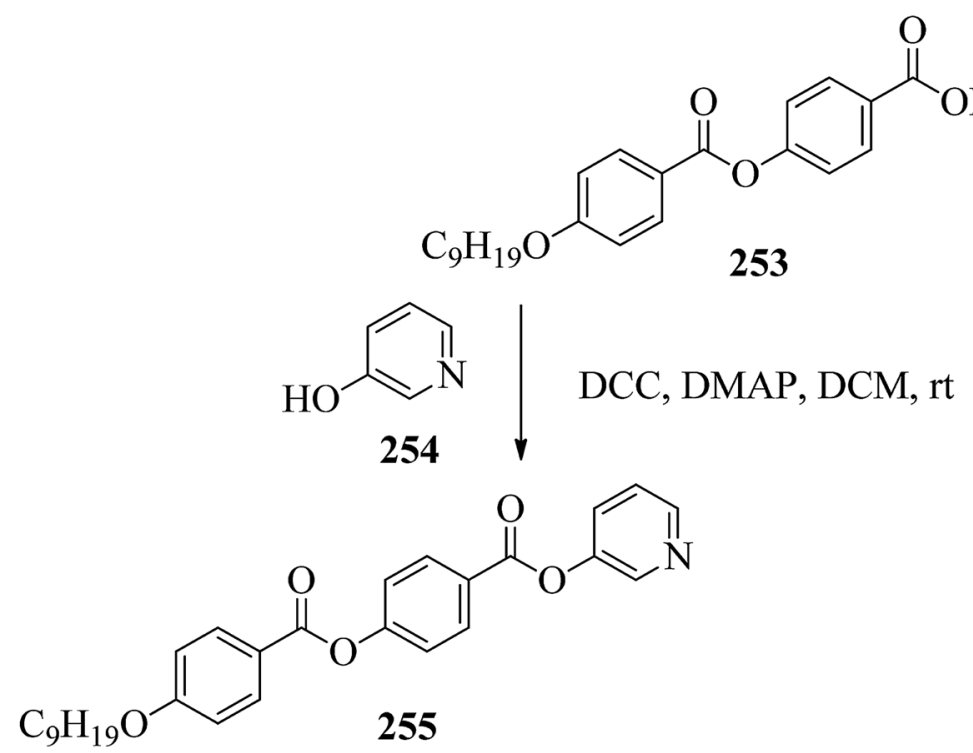

Scheme 34 Synthetic route for the proton acceptor 255
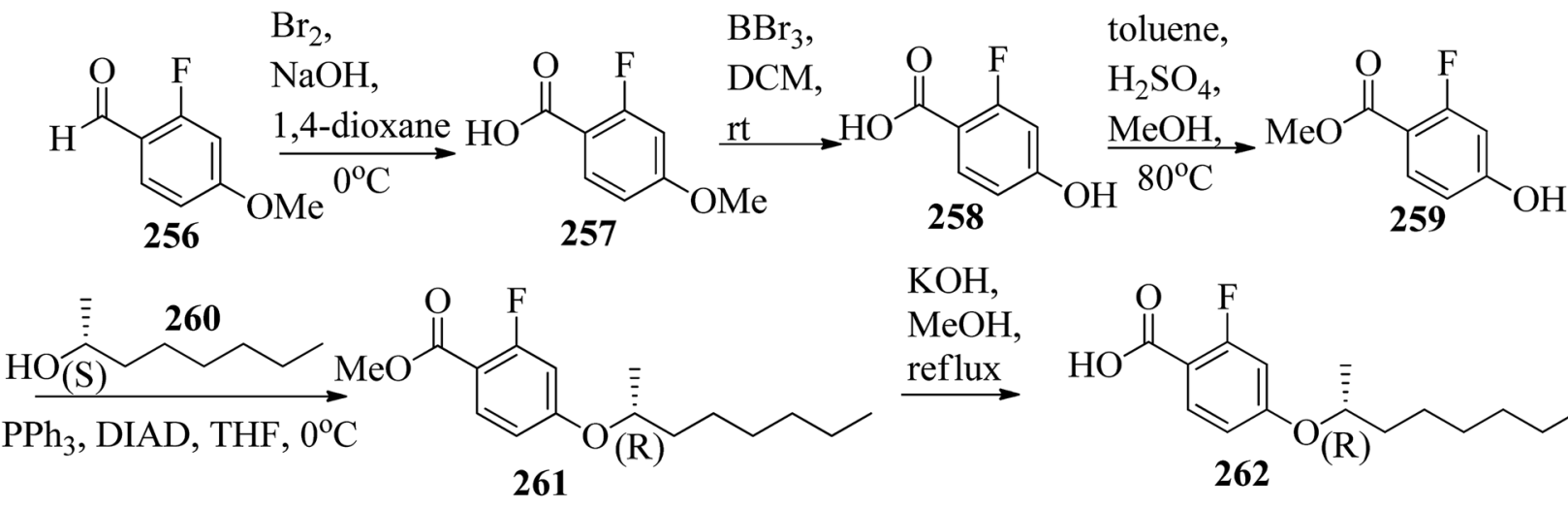

$\mathrm{KOH}$, $\mathrm{MeOH}$,<smiles>C=C(Cc1ccccc1)c1ccc(OC(=O)c2ccc(OCCCC)cc2)cc1</smiles><smiles>O=C(OCc1ccccc1)c1ccc(O)cc1</smiles>

DCC, DMAP, DCM, rt
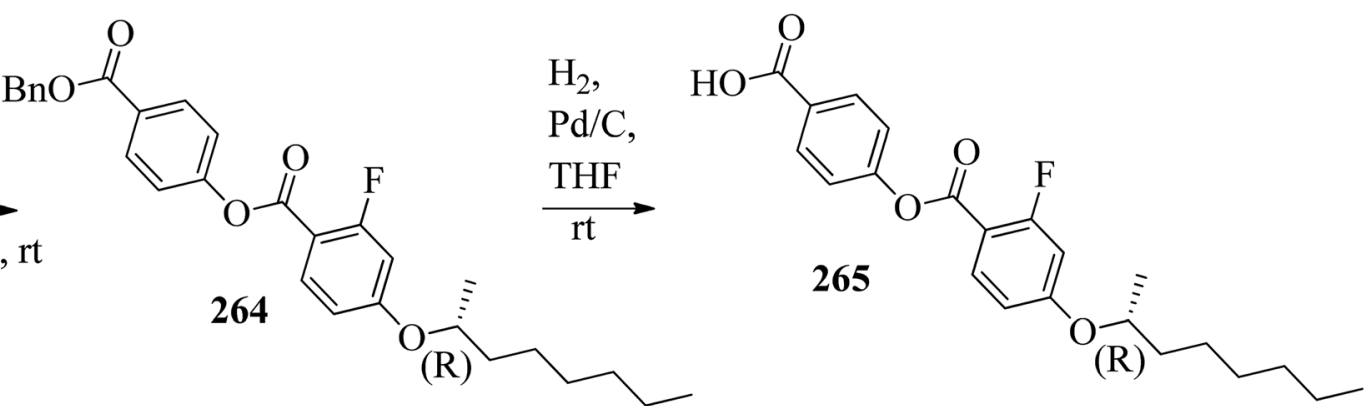
(a)

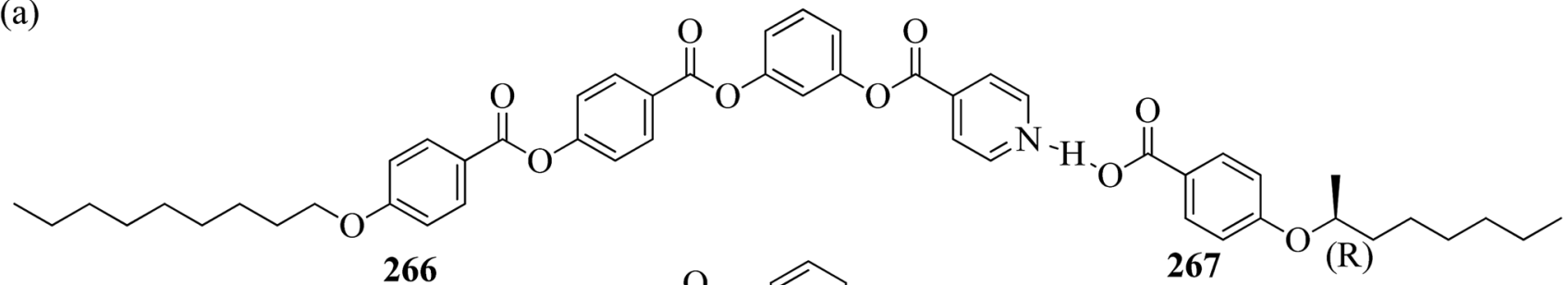

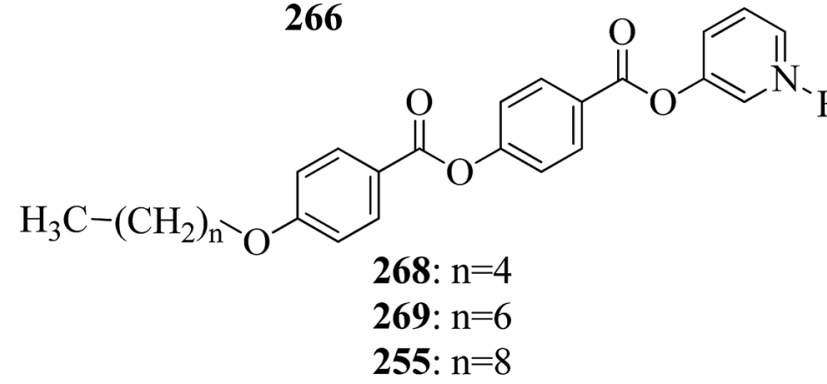<smiles>[X]c1cc(OC([X])CCCCCC)ccc1C(=O)Oc1ccc(C(=O)O)cc1</smiles>

(b)

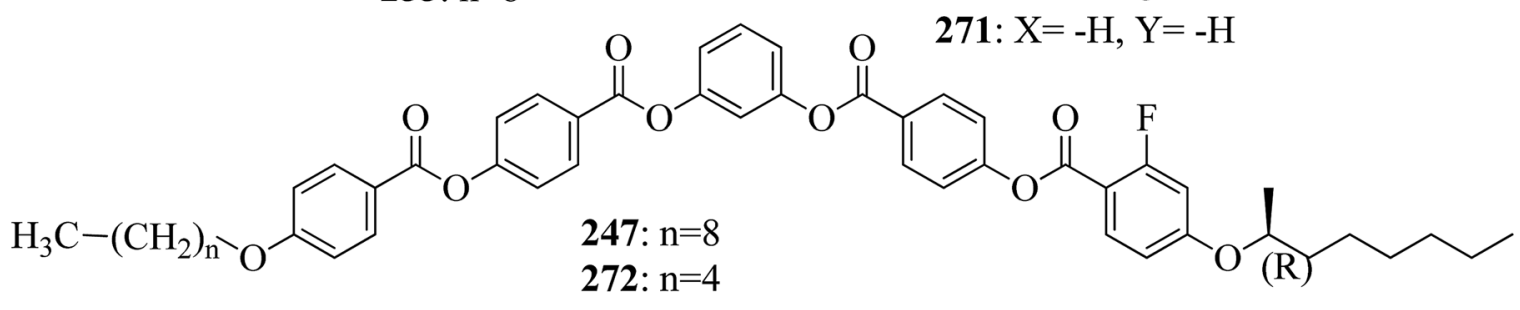

(c)

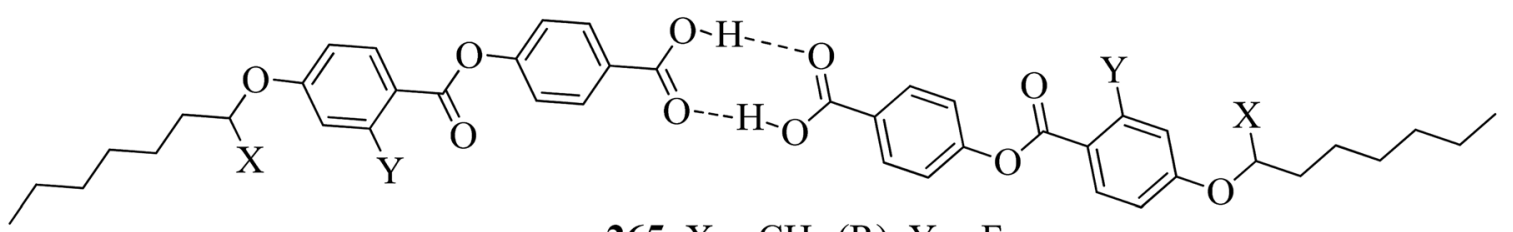

265: $\mathrm{X}=-\mathrm{CH}_{3}(\mathrm{R}), \mathrm{Y}=-\mathrm{F}$

270: $\mathrm{X}=-\mathrm{CH}_{3}(\mathrm{R}), \mathrm{Y}=-\mathrm{H}$

Fig. 15 (a) Structures of the hydrogen bonded complexes; (b) structure of the covalent bonded analogue; (c) structures of the hydrogen bonded dimers.

hence, they reported new transition temperatures in addition to those of the non-reported pyridine-based component 162a. The prepared acids (163a-b) exhibited enantiotropic nematic phase, which was ascribed to dimerisation of the acid molecules. The short chain pyridine-based components (162a-b) exhibited nematic and smectic A phases; however, as the chain length increased (162c-d), only smectic A phase was observed by quenching the nematic phase. The intermolecular hydrogen bond formation between the acid and base elements induces broad-ranged nematic phases by quenching the smectic A phases of the pyridine-based derivatives in all the prepared complexes. The nematic phase was extended over a wide range of temperature; this range even overshot $\sim 75 \mathrm{~K}$ for most of the aggregates and reached $\sim 90 \mathrm{~K}$ for $\mathbf{1 6 2} \mathbf{b} / \mathbf{1 6 3 b}$. This indicates that in these supramolecular structures, the nematic phase is stabilized by hydrogen bonding. For all the prepared aggregations, the nematic mesophases could be super-cooled prior to crystallization, with the highest value of $\sim 48 \mathrm{~K}$ for $162 \mathrm{~d} / \mathbf{1 6 3 b}$ and the lowest value of $\sim 23 \mathrm{~K}$ for 162a/163b.
Chen et al. ${ }^{50}$ synthesized mesogens with 4-pyridyl end groups containing phenylenes and one triple bond. The compounds 4(2-(trans-4- $n$-alkylcyclohexyl)ethyl)iodobenzene (168a-d) were synthesized according to the previous literature strategy used by Chen et al. ${ }^{74}$ To a suspension of $\mathrm{Pd}\left(\mathrm{PPh}_{3}\right)_{4}$, 4-iodopyridine (164),

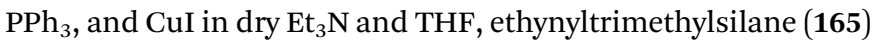
in dry $\mathrm{Et}_{3} \mathrm{~N}$ was added dropwise at room temperature. The reaction mixture was stirred at $60{ }^{\circ} \mathrm{C}$ for $8 \mathrm{~h}$ under nitrogen protection. After that, it was cooled and filtered; then, DCM solvent was added and the organic layer was washed using saturated ammonium chloride solution. Later, the solution was dried using anhydrous $\mathrm{MgSO}_{4}$ and the solvents were evaporated. The obtained crude product was directly utilized for the next steps. This crude product was dissolved in methanol and taken in a nitrogen-flushed round-bottom flask; then, $\mathrm{K}_{2} \mathrm{CO}_{3}$ was added. The reaction mixture was stirred for $3 \mathrm{~h}$ and filtered through Celite. Then, the solvent was dried under reduced pressure to yield 4-ethynylpyridine (167), and it was further purified by column chromatography using petroleum ether as the eluent. To a mixture of $\mathrm{K}_{2} \mathrm{CO}_{3}$, water and 4-pyridineboronic 


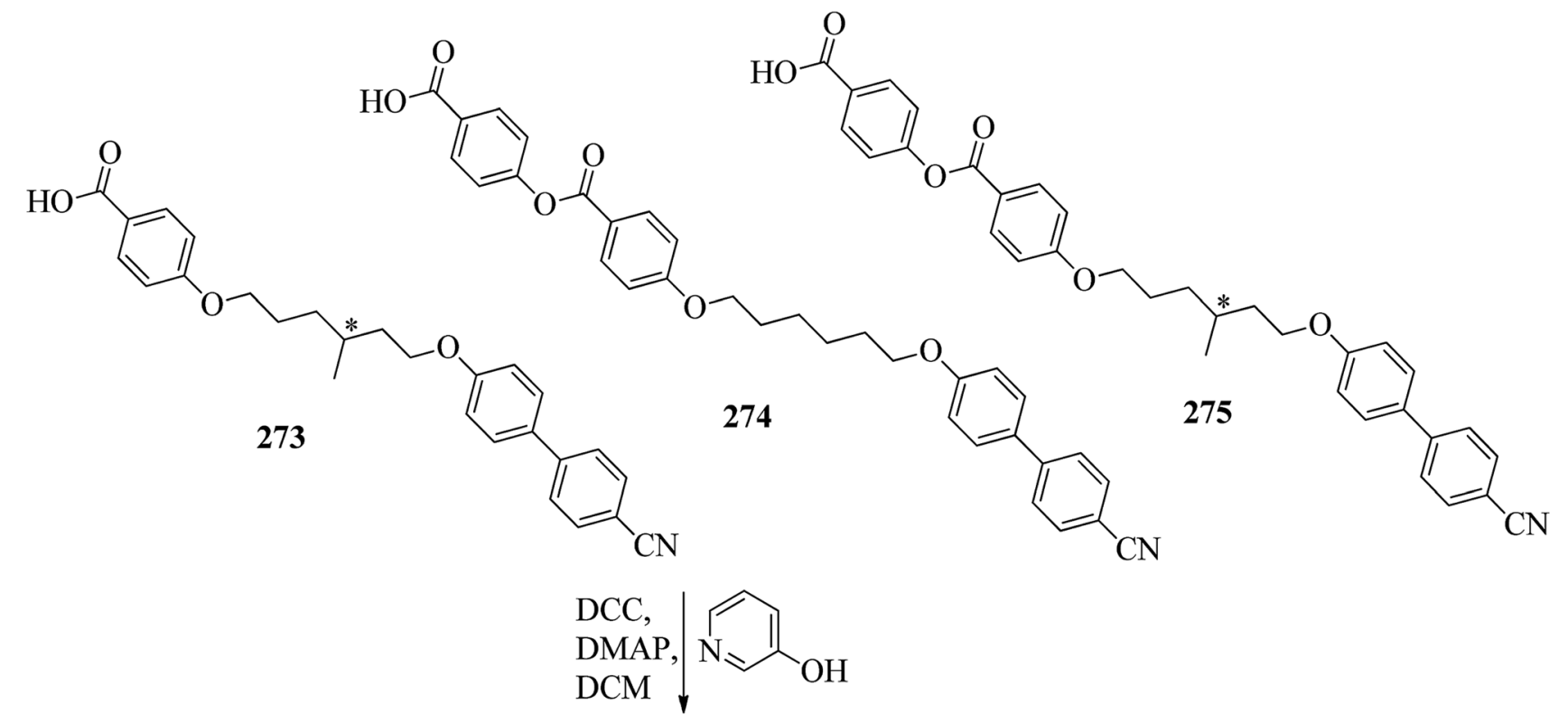<smiles>CC(CCCOc1ccc(-c2ccc(C#N)cc2)cc1)CCOc1ccc(C(=O)Oc2ccc(C(=O)Oc3ccc(C(=O)Oc4ccc(C(=O)Oc5ccc(-c6ccc(OCCC[C@H](C)CCOc7ccc(-c8ccc(C#N)cc8)cc7)cc6)cc5)cc4)cc3)cc2)cc1</smiles>

Scheme 36 Synthesis of hydrogen acceptors 276-278.

acid (171) in DMF, 1-bromo-4-iodobenzene (170) in DMF was added. This reaction mixture was freed from unwanted gas using $\mathrm{N}_{2}$ for $10 \mathrm{~min}$, and then $\operatorname{Pd}\left(\mathrm{PPh}_{3}\right)_{4}$ was added to it. This reaction mixture was maintained with stirring under $\mathrm{N}_{2}$ at $60^{\circ} \mathrm{C}$ for $15 \mathrm{~h}$. After the reaction was complete, the solvent was evaporated using a rotary evaporator; then, the precipitate was dissolved in $\mathrm{CH}_{2} \mathrm{Cl}_{2}$ solvent and washed with water and brine solution. The organic layer was then dried using magnesium sulphate, filtered and concentrated to yield 4-(4-bromophenyl) pyridine (172). Similar to the synthetic process of 166, compound $\mathbf{1 7 2}$ was used as the raw material to afford the product 4-(4-((trimethylsilyl)ethynyl)phenyl)pyridine. Then, this product was dissolved in methanol, and $\mathrm{K}_{2} \mathrm{CO}_{3}$ was added to it. The reaction mixture was then stirred for $4 \mathrm{~h}$, and the reaction mixture was extracted using DCM, washed with brine and water, and dried using $\mathrm{MgSO}_{4}$. Under reduced pressure, the solvent was evaporated to obtain the compound 4-(4-ethynylphenyl) pyridine (173). A suspension of $\mathrm{PdCl}_{2}\left(\mathrm{PPh}_{3}\right)_{2}, \quad$ 2-(4bromophenyl)-4,4,5,5-tetramethyl-1,3,2-dioxaborolane (175),

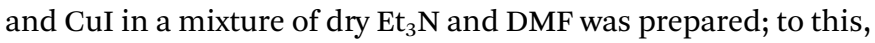
4-ethynylpyridine (177) in $\operatorname{dry~}^{2} \mathrm{Et}_{3} \mathrm{~N}$ was added dropwise, and stirring was maintained at $100{ }^{\circ} \mathrm{C}$ for $8 \mathrm{~h}$. After the reaction was complete, the solvent was removed to obtain $4-((4-(4,4,5,5-$ tetramethyl-1,3,2-dioxaborolan-2-yl)phenyl)ethynyl)-pyridine (176) as the product. A mixture of compound 177, THF and $\mathrm{Et}_{3} \mathrm{~N}$ was prepared in a two-way flask and bubbled with $\mathrm{N}_{2}$ gas. 4-(2(trans-4- $n$-Propylcyclohexyl)ethyl)iodobenzene, CuI, $\mathrm{Pd}\left(\mathrm{PPh}_{3}\right)_{4}$, THF were taken in another two-way flask, which was filled with $\mathrm{N}_{2}$. To this reaction mixture, they added the previous liquid mixture. Later, this reaction mixture was stirred for $8 \mathrm{~h}$ at $60^{\circ} \mathrm{C}$. They used saturated ammonium chloride solution to remove CuI from the reaction mixture. Using water, the mixture was diluted and extracted with ethyl acetate. The organic layer was then dried using $\mathrm{MgSO}_{4}$ and the solvents were evaporated in 
<smiles>CCCCCC[C@H](C)Oc1ccc(C(=O)O)c(F)c1</smiles>

279<smiles>CCCCCC[C@H](C)Oc1ccc(C(=O)O)cc1</smiles>

280<smiles>CCCCCCCOc1ccc(C(=O)O)c(F)c1</smiles>

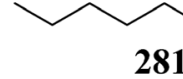<smiles>CCCCCC[C@H](C)Oc1ccc(C(=O)Oc2ccc(C(=O)O)cc2)c(F)c1</smiles>
282<smiles>CCCCCCCOc1ccc(C(=O)O)c(F)c1</smiles>

283<smiles>O=C(OCc1ccccc1)c1ccc(O)cc1</smiles>

(1) DCC, DMAP, DCM, rt (2) $\mathrm{H}_{2}, \mathrm{Pd} / \mathrm{C}, \mathrm{THF}, \mathrm{rt}$<smiles>CCCCCC[C@H](C)Oc1ccc(C(=O)Oc2ccc(C(=O)O)cc2)cc1C(=O)Oc1ccc(C(=O)O)cc1</smiles><smiles>C=S(=O)(O)c1ccc(OC(=O)c2ccc(OCCCCCCC)cc2F)cc1</smiles>

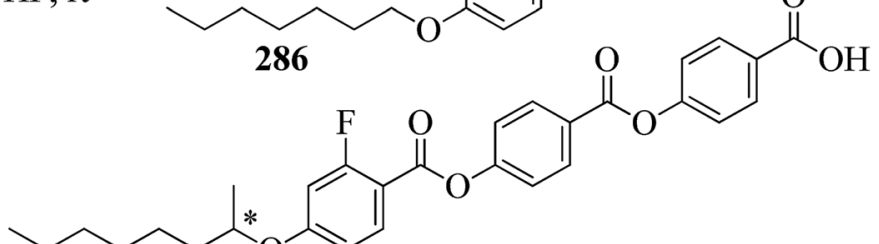<smiles>C/C=C\C(=[18O])O/C(C)=C\CCCCCC</smiles>

Scheme 37 Synthesis of hydrogen donors $284-288$

(a)<smiles>[Y]c1cc(OC([X])CCCCCC)ccc1C(=O)Oc1ccccc1</smiles><smiles>CC(=O)Oc1cccnc1</smiles>

289: $X=-H, Y=-F, Z=-H$

288

(b)<smiles>CCCCCC(C)Oc1ccc(C(=O)Oc2ccc(C(=O)Oc3ccccc3)cc2)c(F)c1</smiles>

290: $X=-\mathrm{CH}_{3}, Y=-\mathrm{H}, \mathrm{Z}=-\mathrm{H}$

291: $\mathrm{X}=-\mathrm{CH}_{3}, \mathrm{Y}=-\mathrm{F}, \mathrm{Z}=-\mathrm{H}$

292: $X=-H, Y=-F, Z=-\mathrm{CH}_{3}$

293: $\mathrm{X}=-\mathrm{CH}_{3}, \mathrm{Y}=-\mathrm{H}, \mathrm{Z}=-\mathrm{CH}_{3}$

294: $\mathrm{X}=-\mathrm{CH}_{3}, \mathrm{Y}=-\mathrm{F}, \mathrm{Z}=-\mathrm{CH}_{3}$<smiles>[Z]C(CCCOC)CCOc1ccc(-c2ccc(C#N)cc2)cc1</smiles>

295: $X=-H, Y=-H, Z=-\mathrm{CH}_{3}$

Fig. 16 (a) Structures of hydrogen bonded complexes 289-295; (b) structure of hydrogen bonded complex 296. 
<smiles>CC(=O)Oc1ccc(/C=C/c2cccc(/C=C/c3ccc(OC(C)=O)cc3)n2)cc1</smiles>

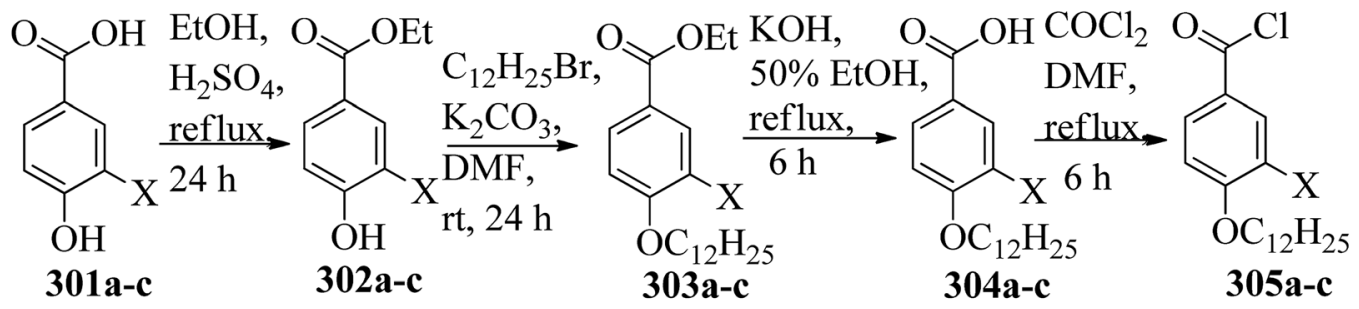

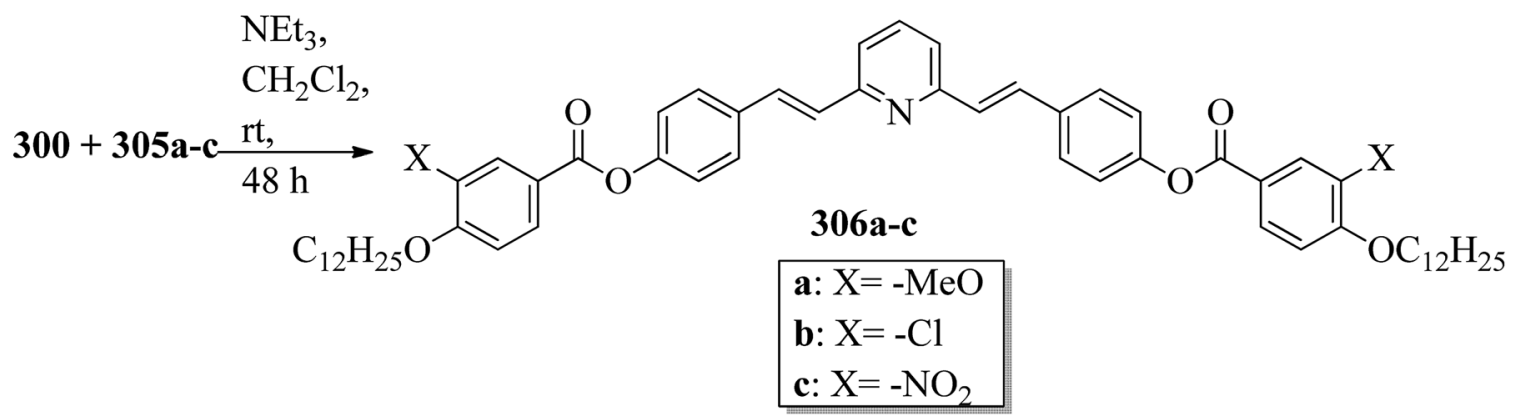

Scheme 38 Synthetic route for compounds 306a-c<smiles>CCCCCCCCCOc1ccc(C(=O)Oc2ccc(/C=C/c3cccc(/C=C/c4ccc(OC(=O)c5ccc(OCC)cc5)cc4)n3)cc2)cc1</smiles>

Fig. 17 Structure of compound 124p, which was reported by Marković et al. ${ }^{107}$

vacuum to yield 4-((4-(2-(trans-4-n-propylcyclohexyl)ethyl) phenyl)ethynyl)pyridine (169b); further, it was purified by column chromatography. Also, the authors synthesized 4-(4-((4(2-(trans-4-npropylcyclohexyl)ethyl)phenyl)ethynyl)-phenyl) pyridine (174b) similarly to $169 \mathrm{~b}$ by replacing 167 with 173 . To a mixture of $\mathrm{K}_{2} \mathrm{CO}_{3}$ in water and $\mathbf{1 7 6}$ in DMF, they added $\mathbf{1 6 8 \mathrm { b }}$ in DMF. Using $\mathrm{N}_{2}$, this solution was degassed for $10 \mathrm{~min}$, and then $\operatorname{Pd}\left(\mathrm{PPh}_{3}\right)_{4}$ was added to it. This reaction mixture was maintained with stirring for $12 \mathrm{~h}$ under $\mathrm{N}_{2}$ at $60{ }^{\circ} \mathrm{C}$ to obtain the product $\quad 4-\left(\left(4^{\prime}-\left(2-\left(\right.\right.\right.\right.$ trans-4-npropylcyclohexyl)ethyl)-[1, $1^{\prime}-$ biphenyl]-4-yl)-ethynyl)pyridine (177b). Using similar procedures, the other target compounds were synthesized (Scheme 42). From the study of these synthesized compounds, they observed that compounds 169a-d exhibited nematic phase ranges of $8.8^{\circ} \mathrm{C}$ to $17.9{ }^{\circ} \mathrm{C}$ and $9.1^{\circ} \mathrm{C}$ to $39^{\circ} \mathrm{C}$ on heating and cooling, respectively, while compounds 174a-d exhibited ranges of $82.9^{\circ} \mathrm{C}$ to $95.5^{\circ} \mathrm{C}$ and $83.7^{\circ} \mathrm{C}$ to $111.5^{\circ} \mathrm{C}$ on heating and cooling, respectively, and compounds 177a-d exhibited ranges of $73.6^{\circ} \mathrm{C}$ to $77.8^{\circ} \mathrm{C}$ on heating and cooling, respectively. The small molecular length-to-width ratios led to nonmesogenic natures of compounds 169a-d with short terminal chains $(n<4)$. The clearing temperatures of compounds 169ad increased with increasing terminal alkyl chain length $(n=4$, 5), and the melting temperatures of compounds 169a-d displayed an even-odd effect: higher melting temperatures were observed for compounds 169a-d with odd terminal alkyl chains $(n=3,5)$ than for those with even terminal alkyl chain lengths $(n=2,4)$. At the same time, compound 174a exhibited a lower clearing point and melting point than compound $\mathbf{1 7 4 b}$, but the longer terminal chain lengths $(n=4,5)$ showed lower melting and clearing temperatures. Also, they observed that the alkyl chain length exhibited a small impact on the clearing and melting points of compounds $\mathbf{1 7 7 a - d}$. In addition, an opposite even-odd effect was observed in the clearing and melting points 


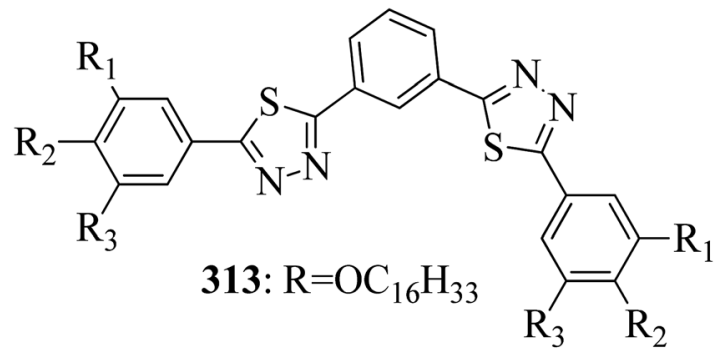<smiles>[R1]c1cc(-c2nnc(-c3cccc(-c4nnc(-c5cc([R])c([R4])c([R1])c5)o4)c3)o2)cc([R])c1[R2]</smiles>

Fig. 18 Structures of previously reported benzene-based molecules 313 and $314 a-b$.

of compounds 177a-d. They also investigated the impact of another phenyl rigid core on the mesogenic properties. The stability of the nematic phase was improved by adding a phenyl rigid core to the mesogen core to afford compounds $\mathbf{1 7 4 b}$ and $177 \mathrm{~b}$, in which the nematic phase ranges increased from $0{ }^{\circ} \mathrm{C}$ to $94.7^{\circ} \mathrm{C}$ and $0{ }^{\circ} \mathrm{C}$ to $77.9^{\circ} \mathrm{C}$, respectively. Further, these results revealed that the introduction of another phenyl rigid system induces nematic phase formation and enhances the nematic phase interval. Moreover, there is a $38.3^{\circ}$ twist in the biphenyl rings of compound $\mathbf{1 7 7 b}$ and twist angles of about $36.9^{\circ}$ in the phenyl and pyridine rings in compound $\mathbf{1 7 4 b}$; this influences<smiles>Oc1ccccc1</smiles>

315<smiles>Oc1ccc(O)cc1</smiles><smiles>Oc1ccc(O)c(O)c1</smiles>

316<smiles>Oc1cccc(O)c1O</smiles>
319<smiles>Oc1ccc(O)c(O)c1</smiles><smiles>Oc1cc(O)cc(O)c1</smiles>

320

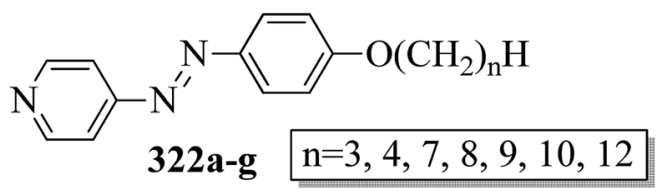

Fig. 19 Structures of the core units 315-321 and the proton acceptors $322 a-g$. the broadening of the nematic mesophase intervals. In addition, nematic mesophase stability was favored by the adequate dipole-dipole interactions of compound 174b (Scheme 21).

Walker et al. ${ }^{75}$ prepared hydrogen bonded complexes using alkoxy benzoic acids (186a-b) and a stilbazole-based template (185) as the proton donor and acceptor, respectively. Referring to the procedures described by Abberley et al. ${ }^{76}$ they synthesized the compound 4-(6-bromohexyl)-4'-methoxy-1,1'-biphenyl (181). Then, they prepared 4-[(E)-2-(pyridin-4-yl)ethenyl]phenol (184) by refluxing a reaction mixture containing 4-methylpyridine (183) and 4-hydroxybenzaldehyde (182) in acetic anhydride for $23 \mathrm{~h}$. After completion of the reaction, the reaction mixture was cooled to room temperature, poured into ice water and stirred for $1 \mathrm{~h}$. The precipitate was filtered and refluxed for $2 \mathrm{~h}$ in the presence of alcoholic potassium hydroxide. To obtain compound $\mathbf{1 8 4}$ as a precipitate, they added acetic acid; the precipitate was then recrystallized using ethanol solvent. Compound 184, compound 181 and potassium carbonate were dissolved in acetone and refluxed for $96 \mathrm{~h}$ at $65{ }^{\circ} \mathrm{C}$. After completion of the reaction, the reaction mixture was cooled to room temperature; the formed precipitate was filtered and purified using column chromatography to yield $4-[(E)-2-(4-\{[6-$ (4'-methoxy[1,1'-biphenyl]-4-yl)hexyl]oxy\}phenyl)ethenyl]

pyridine (185). The synthetic route for compound 185 is illustrated in Scheme 22. Finally, the expected hydrogen bonded binary mixtures were prepared by dissolving equimolar ratios of 185 and 186a or 186b in dichloromethane, and the solvent was evaporated at room temperature. Then, the mixture was maintained in a vacuum oven for $16 \mathrm{~h}$ at $50{ }^{\circ} \mathrm{C}$ for drying (Scheme 23). The aggregations exhibited various mesophases across a wide range of temperatures even though the parent molecule 185 is a non-mesogen and 186a-b, with $n=4$ or 5 , display only narrow temperature ranges of achiral nematic mesophase. During the cooling cycle, the 187a aggregation displayed two nematic mesophases that were differentiated by their optical textures: in cells with planar securing, the higher-level temperature nematic mesophase displays an absolutely uniform texture, and at the lower transition temperature of mesophase, a stripe-like texture develops in typical twist-bend nematic mesophase. The emergence of the twist-bend nematic mesophase was accompanied by the termination of the optical glimmering ascribed to the director variations noted in the nematic mesophase. The nematic to twist-bend nematic mesophase transition was observed in DSC analysis as a minute leap in the heat capacity. This was in agreement with the normal result that as the temperature range preceding nematic mesophase increases, the nematic to twist-bend nematic mesophase transition tends to become second order in nature, i.e. $\Delta H \approx 0$. In thin cells, the sample crystallization was arrested and a reversible twist-bend nematic mesophase to smectic $\mathrm{X}$ transition was observed. Unfortunately, the monotropic character of this phase hindered its analysis using XRD; however, given the high enthalpy variation linked with the transition, it was presumed to be a smectic phase of high order. For the $\mathbf{1 8 7 b}$ aggregation, the temperature range of the twist-bend nematic mesophase decreased to $\sim 5 \mathrm{~K}$, which was inadequate for the development of the stripe-like texture. Further, smectic A and 


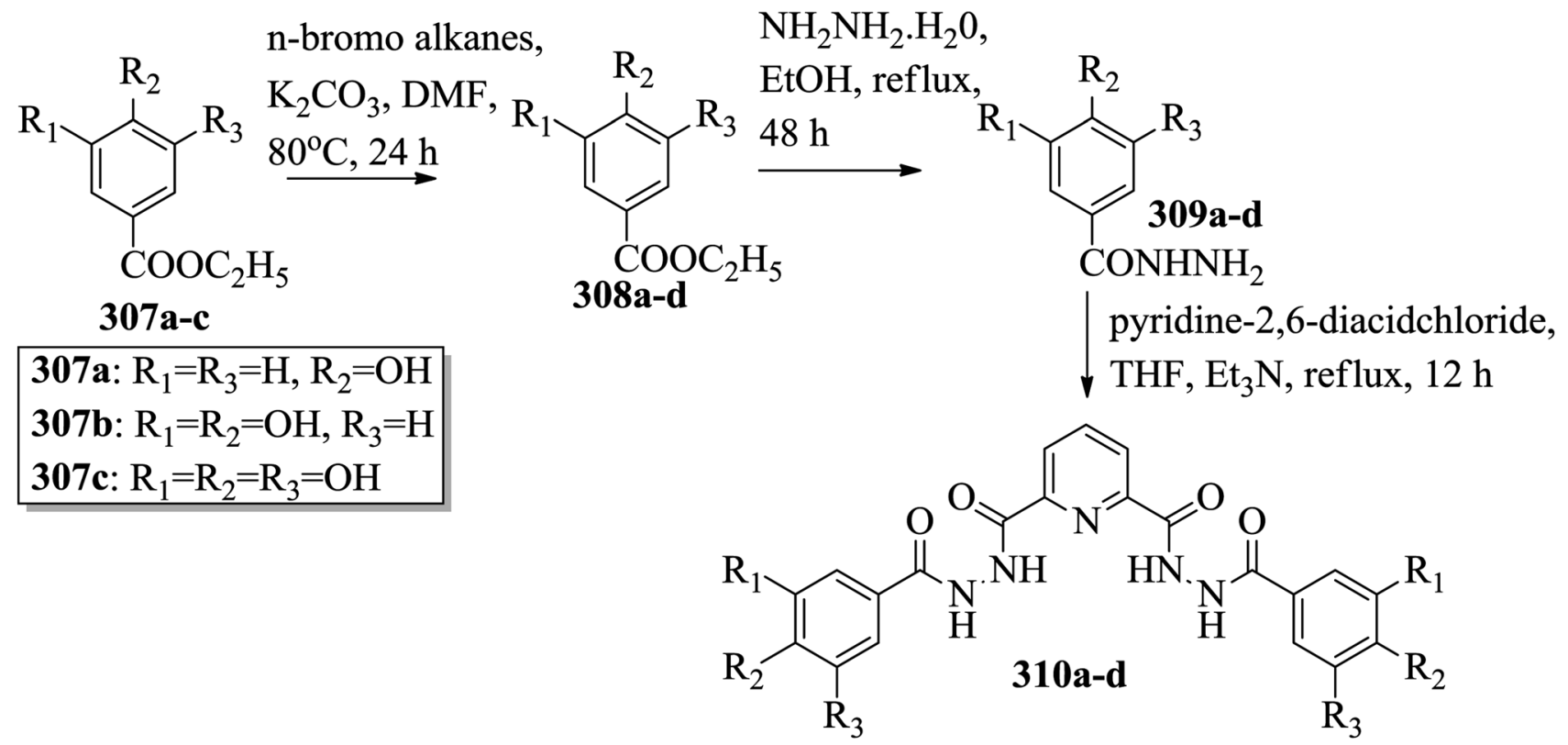

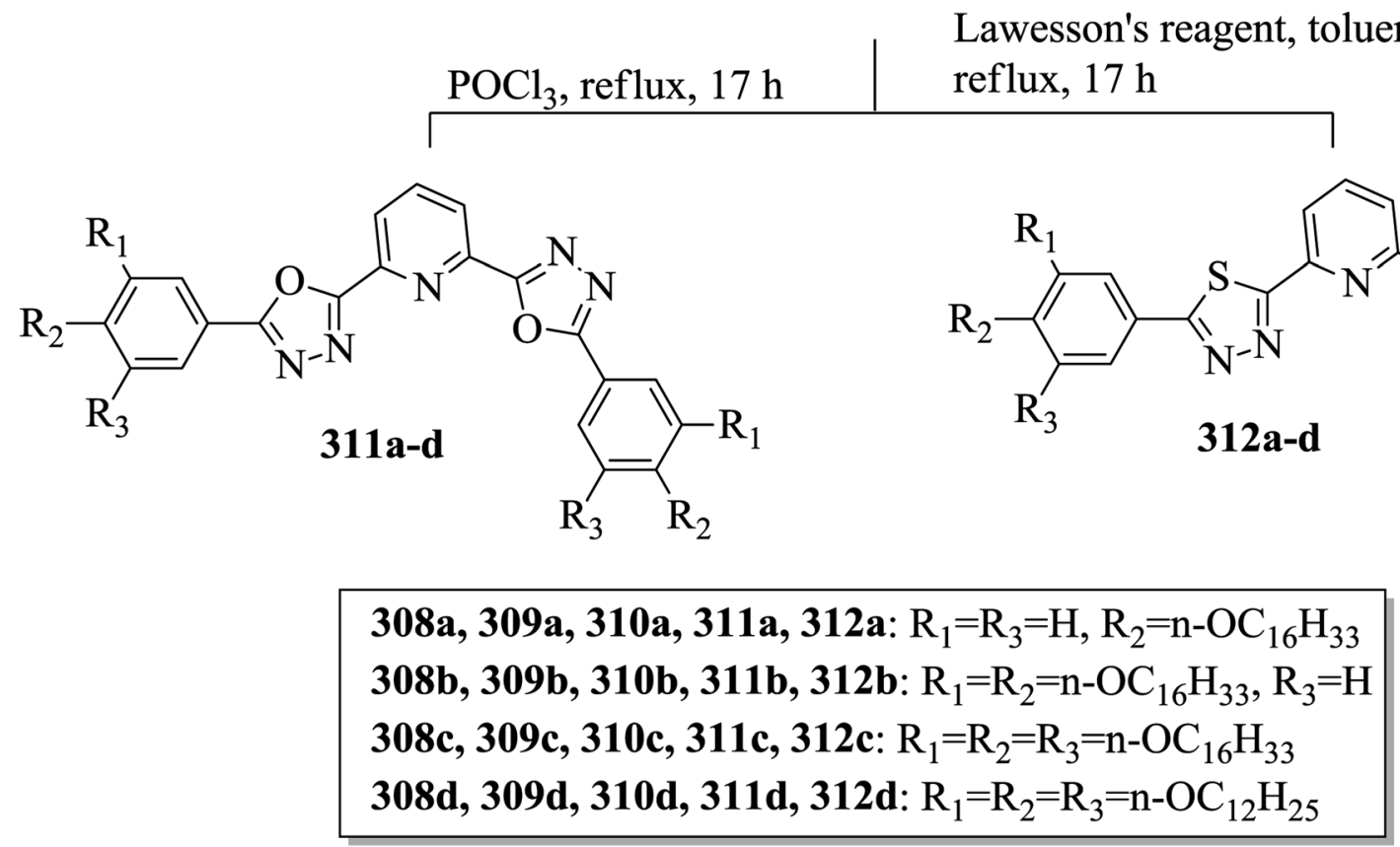

Scheme 39 Preparation of $311 a-d$ and $312 a-d$

smectic X phases were noted below the twist-bend nematic mesophase.

Chakraborty et al. ${ }^{77}$ prepared hydrogen bonded complexes using alkoxy benzoic acid as the proton donor and pyridenyl benzothiazole as the proton acceptor. The synthetic route for the preparation of the hydrogen bonded complexes is illustrated in Scheme 24. The derivative of benzothiazole, i.e. 2(pyridin-4-yl)benzothiazole (190), was synthesized by an altered strategy based on the previous literature report by Maleki and Salehabadi. ${ }^{78}$ According to this procedure, the $o$-amino thiophenol (188), aldehyde (189), and ammonium chloride catalyst were refluxed in ethanol solvent. On the other hand, the hydrogen donors, i.e. alkoxy benzoic acids (191a-d) with different chain lengths, were synthesized by necessary modification of the strategy described by Spring et al. ${ }^{79}$ During synthesis, para-hydroxyl benzoic acid, alkyl bromide, and KOH were refluxed in ethanol, followed by acidification using $\mathrm{HCl}$ solution. Finally, the expected hydrogen bonded aggregations were prepared by dissolving equimolar amounts of 2-(pyridin-4yl)benzothiazole (190) and alkoxybenzoic acids (191a-d) in pyridine and refluxing them. The solvent (pyridine) was reduced and removed in vacuum, and the obtained product was dried using a desiccator (Scheme 24). Among the prepared complexes, only 192a displayed monotropic nematic mesophase on supercooling. The complexes displayed notably lower emergence temperatures for the nematic and isotropic phases compared to 


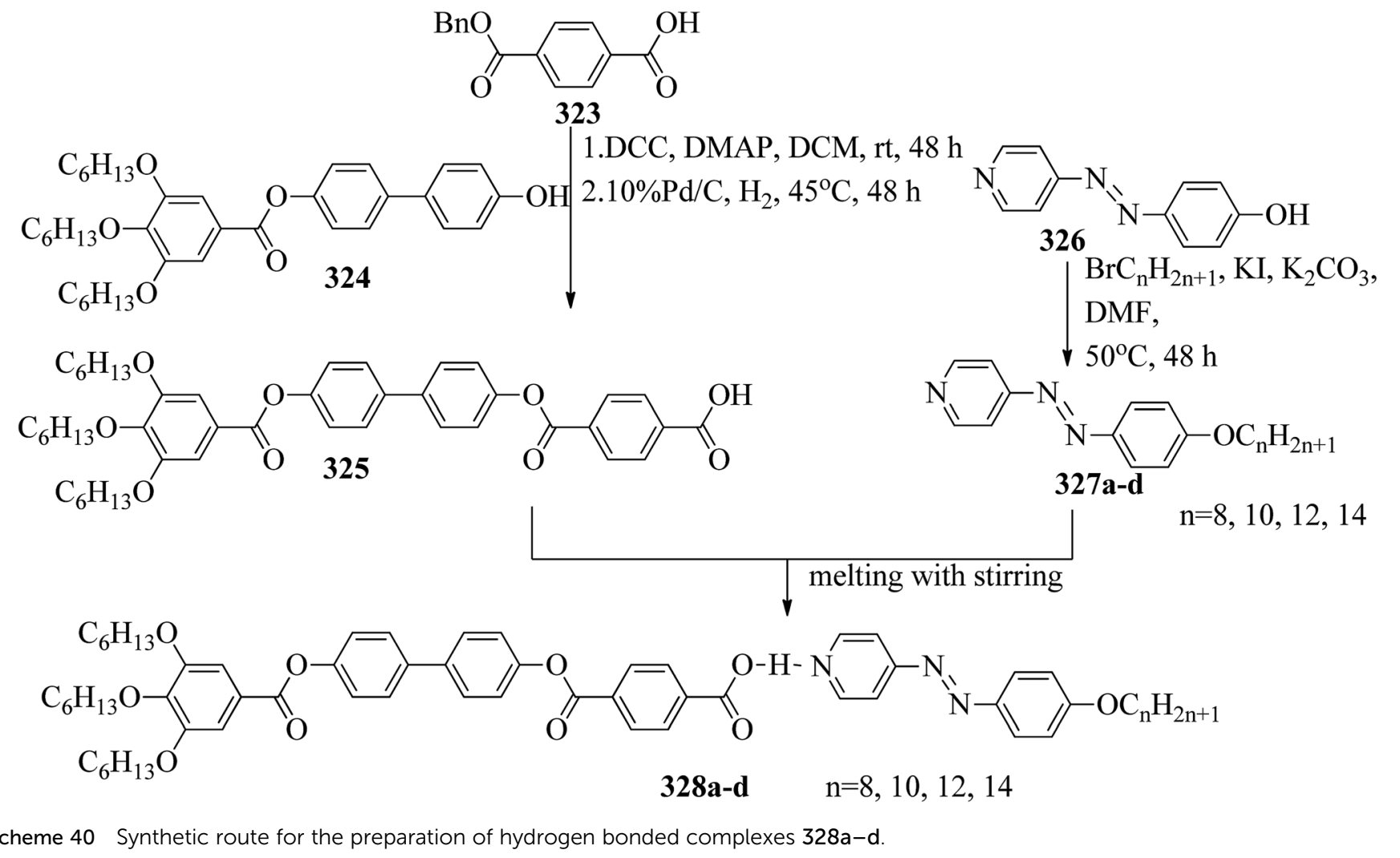

the parent alkoxy benzoic acids. None of the parent alkoxy benzoic acids displayed fluorescence properties when scanned from the crystalline state to mesogenic phase to isotropic phase. Interestingly, the hydrogen bonded complex 192a displayed phase-dependent fluorescence. The aggregates displayed green fluorescence in the crystalline state upon excitation (Ex 300 to $360 \mathrm{~nm}$ filter). During the heating cycle, complex 192a underwent a transition from liquid crystalline to isotropic phase at $97{ }^{\circ} \mathrm{C}$ with completely quenched fluorescence behaviour. During the cooling cycle, moderately increased order in the arrangement of the molecules was observed during the liquid to nematic phase transition stage as well as the appearance of exceptionally low-intensity red fluorescence. Upon further cooling from nematic mesophase to the crystalline state,<smiles>O=C=NCCCCCCNC(=O)[O-]</smiles>

329 $0.02 \mathrm{wt} \%$ Dibutyl Tin Dilaurate
at $80^{\circ} \mathrm{C}$<smiles>CC(C)(C)OCCN(CCOC(=O)NCCCCCCNC(=O)C1(C)CC1)C(=O)c1ccncc1</smiles> 


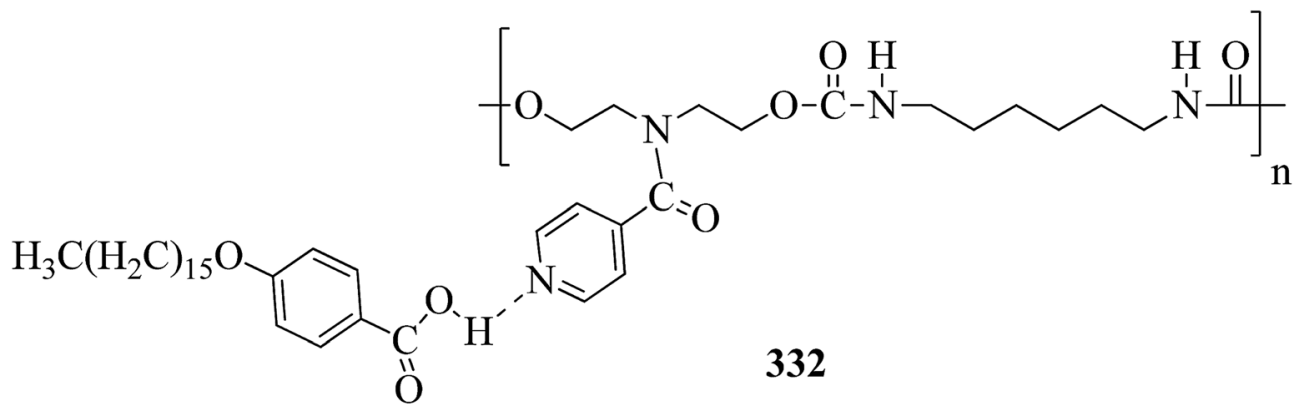

Fig. 20 Supramolecular structure of 332.

the complex displayed bright green fluorescence. Also, further heating/cooling of this mesogen exhibited OFF/ON fluorescence switching. In the crystalline phase, green fluorescence was observed, which is due to the highly ordered alignment of the compound supplied by the strong hydrogen bonds between 190 and 191a. Moderate collapse of the hydrogen bonding between 190 and 191a occurs at high temperature, which causes the intense change in luminescence from bright green to exceptionally low-intensity red fluorescence or non-luminescence.

Goossens and co-workers ${ }^{80}$ published a review article on the design, synthesis, and characterization of certain pyridinium and 4,4'-bipyridinium (viologen)-based thermotropic ionic liquid crystals and also highlighted their applications. Further, Bhowmik et al. ${ }^{81}$ prepared a series of symmetric viologen triflimides obtained by reacting viologen tosyalates with lithium triflimide through metathesis reactions. Furthermore, they noted relatively low melting points of viologen salts that bear two or three carbon atoms in their alkyl chains. In addition, viologen salts carrying alkyl chains with four or five carbon atoms showed ionic liquid behavior at $88{ }^{\circ} \mathrm{C}$ and $42{ }^{\circ} \mathrm{C}$, respectively. Also, the salts of alkyl chains comprising nine, ten or eleven carbon atoms formed high-melting salts. However, mesogenic phases such as smectic $\mathrm{C}$, smectic $\mathrm{A}$ and an unidentified smectic phase (smectic X) were observed only in the viologen salts with higher alkyl chain lengths (i.e. 16, 18 and 20 carbon atoms). In another report (Bhowmik et al..$^{82}$ ), a series of viologens composed of 4 - $n$-alkylbenzenesulfonates were

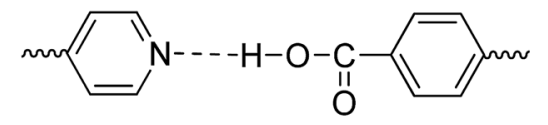

\section{H1 for hydrogen-bonded HOBA}

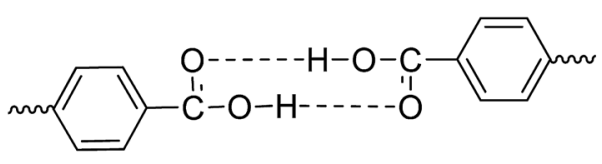

H2 for HOBA dimer prepared by respective metathesis reactions between viologen dibromide and sodium $4-n$-alkylbezenesulfonates or $4-n$-alkylbenzenesulfonic acids. These ionic molecules exhibited focal conic or Schlieren textures (smectic A phases) and undefined smectic X phases with good thermal stability.

Casella and co-workers ${ }^{83}$ synthesized symmetric viologen dimer salts with bistriflimides as counter-ions and investigated the reliance of the mesogenic properties on the lengths of both the lateral and spacer chains. An ordered smectic mesophase (smectic X) was noted for all the compounds. In addition to this, fluid smectic A mesophase was observed when the spacer chain contained less than six carbon atoms and the lateral chains contained more than twelve carbon atoms. Then, Pibiri et al. ${ }^{84}$ prepared two different types of ionic mesogens comprising nonsymmetrically substituted polyfluorinated viologen salts (linear molecules) and bent-shaped symmetrically substituted dialkyloxadiazolyl-bipyridinium salts of bistriflimide ion. Most of the linear molecules displayed smectic X mesophase; in some of the molecules, smectic A mesophase was observed in addition to smectic X mesophase. Moreover, the inserted bent oxadiazolyl spacer between the two pyridinium moieties significantly altered the mesophase properties and exhibited dendritic textures of the banana mesophases.

\subsection{Pyridine-based discotic mesogens}

Discotic liquid crystals are disc-like molecules where the molecules are stacked one above the other to form columns.

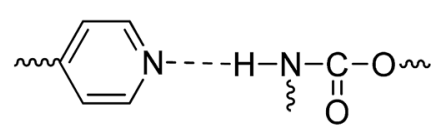

H3 for supramolecular switch<smiles>COC(=O)ON(C)C(=O)OC</smiles>

H4 for physical net point

Fig. 21 Other possible hydrogen bonds in supramolecular complex 332 
Discotic nematic and columnar mesophases are the two main mesophases that are generally observed in this type of mesogen. In the case of discotic nematic mesophase, the molecules possess no positional order but have a least orientational order. On the other hand, the arrangement of molecules in the columns of different lattices gives rise to different types of columnar mesophase patterns, such as columnar rectangular and columnar hexagonal mesophases. This section covers the available synthetic roots for the preparation of different pyridine-based discotic/columnar mesogens and their properties.

Ahipa and Adhikari ${ }^{85}$ synthesized fourteen new blue luminescent mesogens with a core unit of 2-methoxy-3cyanopyridine or 2-methoxypyridine and variable substituted aryl/heteroaryl rings as well as alkoxy phenyls as terminal substituents. The synthesis of the target compounds involves the Claisen-Schmidth reaction, i.e. chalcone formation by reacting ketone (193a-d) and aldehyde (194a-f) compounds in ethanol solvent in the presence of base $(\mathrm{KOH})$ followed by stirring the reaction mixture for $4 \mathrm{~h}$ at room temperature in order to obtain the crude chalcones (195a-n), which are later purified by recrystallization using ethanol solvent. Further, the prepared chalcones were added slowly to freshly prepared sodium methoxide solution and stirred. Then, malononitrile was added, with continuous stirring at room temperature until precipitation of the products $(\mathbf{1 9 6 a}-\mathbf{n})$. Later, the crude products were filtered and washed with methanol, then recrystallized using ethanol (Scheme 25). Further, photophysical studies indicated that these compounds are promising blue emissive materials. Single crystal studies revealed the bent-shaped structure of these compounds, with a marginal non-planar arrangement and various intermolecular interactions. Also, these compounds exhibited liquid crystalline properties, which was confirmed by POM, DSC, and powder XRD techniques. In these compounds, the wide thermal ranges of the mesogens were influenced by the nature of the polar substituents. Nematic phase was also observed in the compounds with a lateral -CN group attached to the pyridine core and a terminal $-\mathrm{F}$ or $-\mathrm{Cl}$ substituent. In the presence of the terminal $-\mathrm{NO}_{2},-\mathrm{Br}$ or 4-pyridyl group and in the absence of the lateral -CN group in the pyridine core, the formation of a rectangular columnar phase was observed. Finally, they concluded that these molecules are emerging blue emissive mesogens with good charge transporting ability and can be utilized further for applications in optoelectronics.

In another report, Ahipa and Adhikari ${ }^{86}$ synthesized a series of cyanopyridine derivatives 204a-e carrying a 3,4-dialkoxyphenyl group and studied their optical and mesogenic properties. The synthetic route involves a one-pot synthesis of cyanopyridone compound 201 using 4-hydroxy benzaldehyde

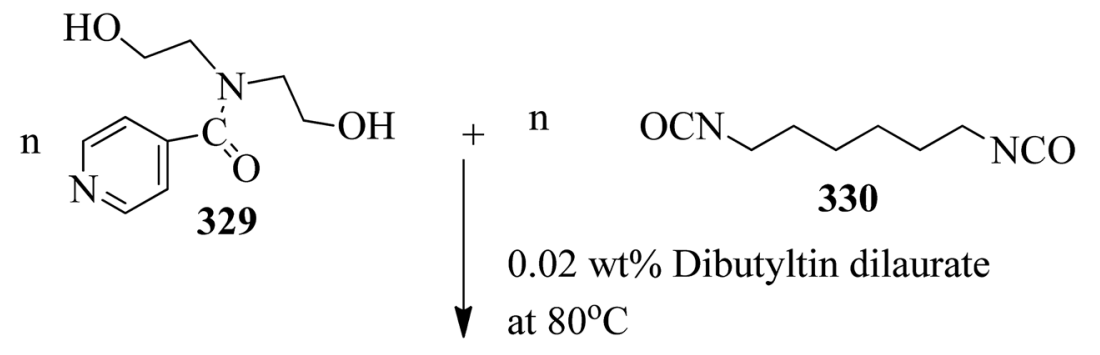<smiles>CC(C)OCCN(CCOC(O)NCCCCCCNC(C)(C)C)C(=O)c1ccncc1</smiles><smiles>CC(=O)Nc1ccc(C(C(=O)O)c2ccc(N=C[O-])cc2)cc1</smiles>

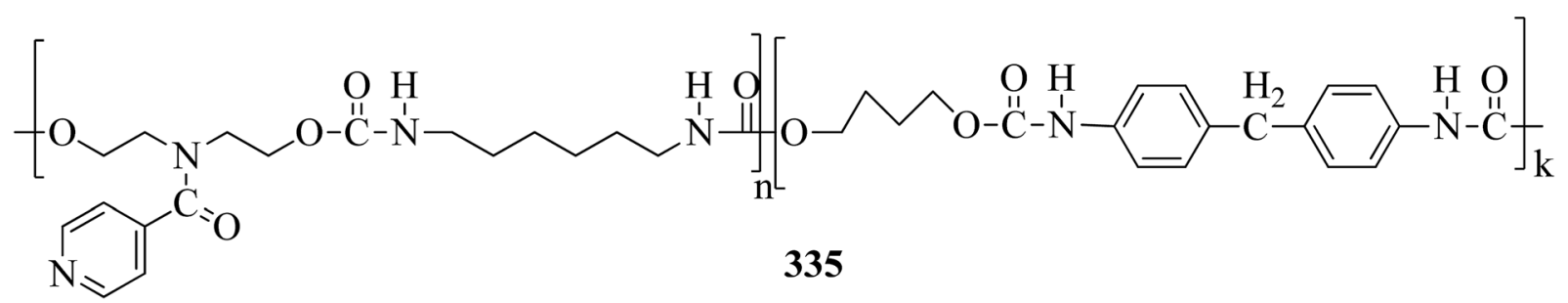

Scheme 42 Synthesis of polymer 335 . 

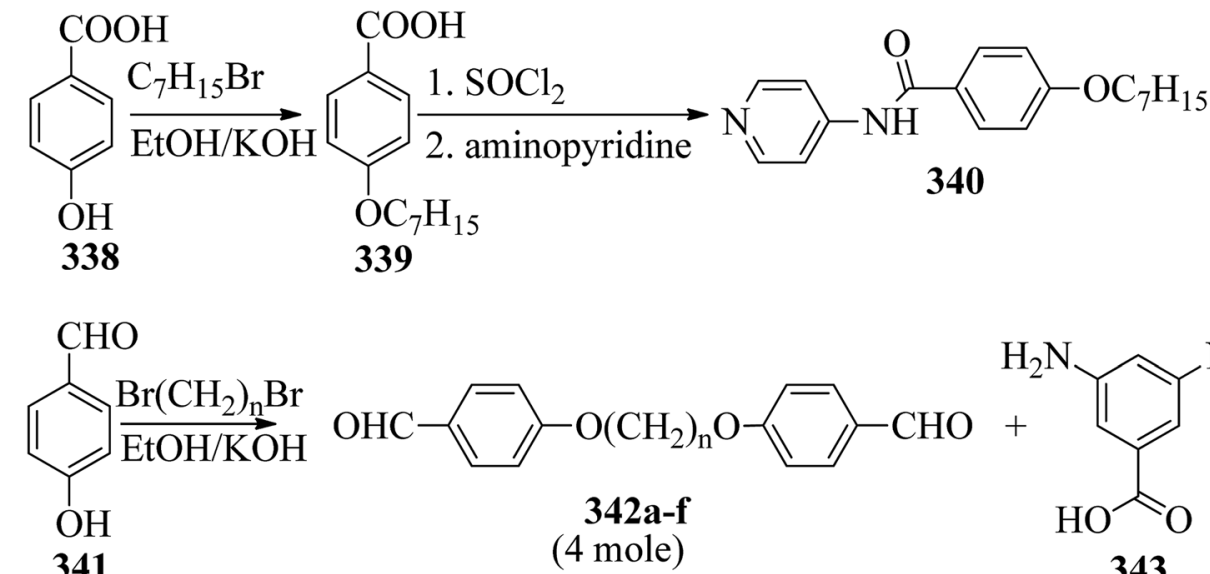<smiles>Nc1cc(N)cc(C(=O)O)c1</smiles><smiles>C/C=C/c1ccc(OC)cc1</smiles><smiles>CCOc1ccc(C=Nc2cc(N=C(CCCCO)c3ccc(OCCOc4ccc(C=O)cc4)cc3)cc(C(=O)O)c2)cc1</smiles>

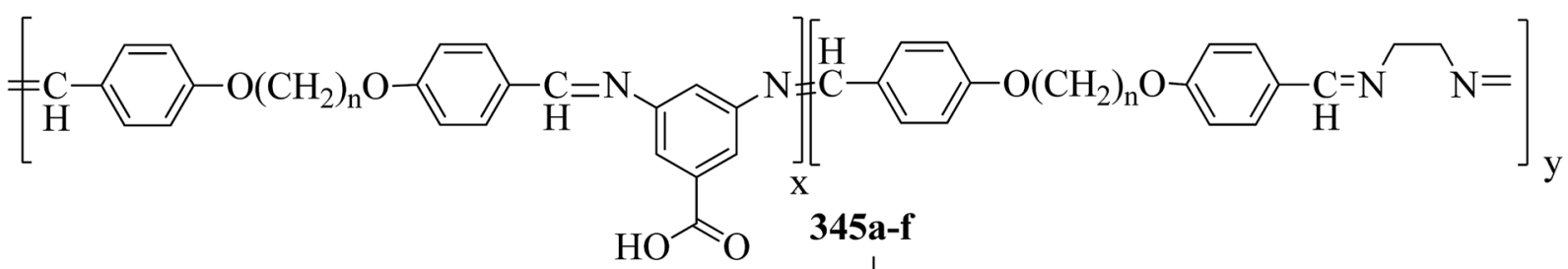

340<smiles>CCCc1ccc(OC)cc1</smiles><smiles>[Y]c1cc(N=C=NCCN=Cc2ccc(O)cc2)cc(C(=O)O)c1</smiles><smiles>CCOc1ccc(C(=O)Nc2ccncc2)cc1</smiles>

Scheme 43 Synthesis of hydrogen bonded complexes $346 a-f$.

(200) and 4-hydroxy acetophenone (199) with ethylcyanoacetate and excess ammonium acetate in 1,4-dioxane solvent. Followed by the preparation of the tri-ester functionalized compound $\mathbf{2 0 2}$ using synthesized compound 201 and ethyl chloroacetate in anhydrous $\mathrm{K}_{2} \mathrm{CO}_{3}$ using DMF as a solvent, the formed product was then converted into trihydrazide compound 203 by 


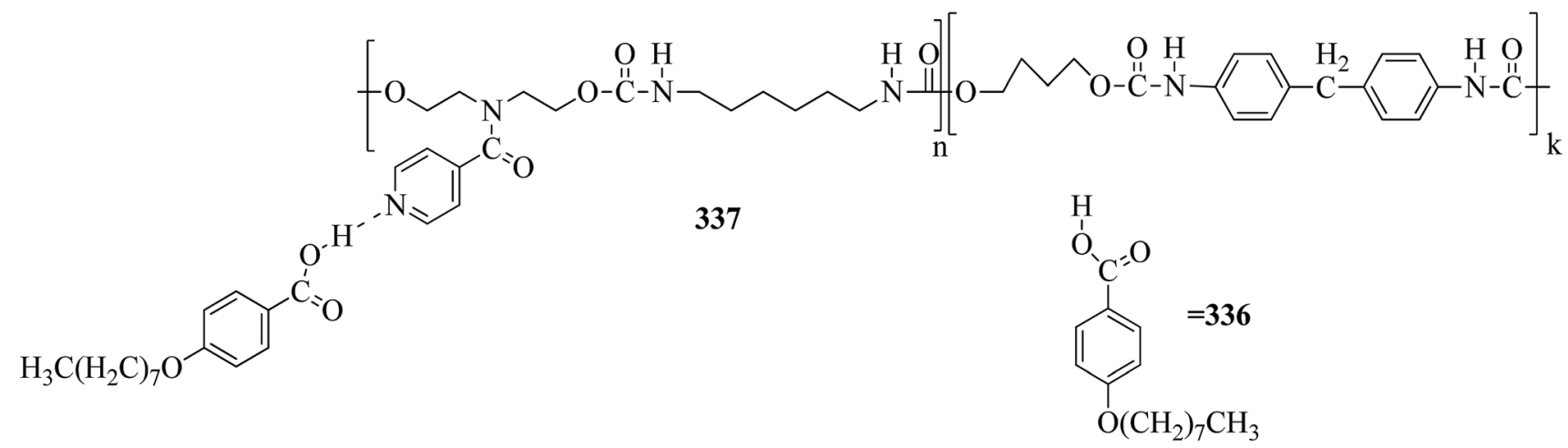

Fig. 22 Hydrogen bonded complex 337.

refluxing with hydrazine hydrate in ethanol solvent. On the other hand, alkylation of 3,4-hydroxy benzaldehyde (197) with various alkyl bromides afforded the 3,4-dialkoxybenzaldehydes 198a-e. Finally, the target products 204a-e were obtained by condensing the prepared 203 and 198a-e in the presence of glacial acetic acid as a catalyst (Scheme 26). The synthesized compounds formed columnar assemblies due to the presence of intermolecular hydrogen bonds and $\pi-\pi$ (core to core) interactions. These compounds exhibited hexagonal columnar mesophase from ambient temperature to $110{ }^{\circ} \mathrm{C}$. The optical studies revealed that all the synthesized compounds exhibited strong blue fluorescence emissions with maxima at around $389 \mathrm{~nm}$, and it was also observed that the photophysical properties of the target compounds were not greatly affected by the terminal alkoxy chain length. An interesting fact is that these target compounds are emitters of blue fluorescence in both the liquid crystalline state and solution state; hence, these compounds are potential candidates for OLED applications.

Further, Ahipa et al. ${ }^{87}$ designed and synthesized compounds with a bent core structure with three rings, i.e. 4-(2-(4alkoxyphenyl)-6-methoxypyridin-4-yl)benzonitriles (208a-f), with variable alkoxy chain lengths. The synthetic route for the synthesis of 208a-f is shown in Scheme 27. The first step involves alkylation of 4-hydroxy acetophenone using alkyl bromides [ $m=4$ to 14 (only even)]. The second step involves the preparation of the required chalcones (207a-f) via ClaisenSchmidt reactions between the alkylated compounds (205a-f) and 4-cyano benzaldehyde (206); finally, the target methoxypyridine derivatives (208a-f) were obtained by cyclising the chalcones and malononitrile at room temperature in the presence of sodium methoxide. During cyclization, the cyano group (electron withdrawing group) attached to the phenyl ring does not facilitate the dehydrogenation process; rather, it undergoes dehydrocyanation. Because of this, the obtained products were methoxy pyridine derivatives, not 4-(4cyanophenyl)-2-methoxy-6-(4-(alkoxy)phenyl)nicotinonitriles (Scheme 27). Further, POM, DSC and powder XRD studies of these synthesized compounds revealed that the compound with the shortest chain length, 208a [i.e. $m=4]$, exhibited nematic phase, while all the other derivatives exhibited orthorhombic columnar phase. Furthermore, the bent-shaped molecular structure of the target compound was readily confirmed by single crystal X-ray analysis. From the optical studies, it was observed that these compounds are blue emitting materials showing absorption and emission bands in the ranges of 335 to $345 \mathrm{~nm}$ and 415 to $460 \mathrm{~nm}$, respectively. From the electrochemical studies, a band gap of $1.89 \mathrm{eV}$ and HOMO and LUMO energy levels of -5.06 and $-3.17 \mathrm{eV}$, respectively, were observed for the compound 4-(2-(4-octyloxyphenyl)-6-methoxypyridin-4yl)benzonitrile (208c).

Lee et al. ${ }^{88}$ prepared discotic liquid crystals $(211 \mathrm{a}-\mathrm{c})$ by the formation of hydrogen bonding between the discotic core 1,3,5cyclohexanetricarboxylic acid (209) and the peripheral units of stilbazole derivatives $(\mathbf{2 1 0 a}-\mathbf{c})$. The three stilbazole derivatives, i.e. 4-(4-decyloxybenzoyloxy)-4'-stilbazole (210a), 4-(3,4-didecyloxybenzoyloxy)-4'-stilbazole (210b), and 4-(3,4,5-

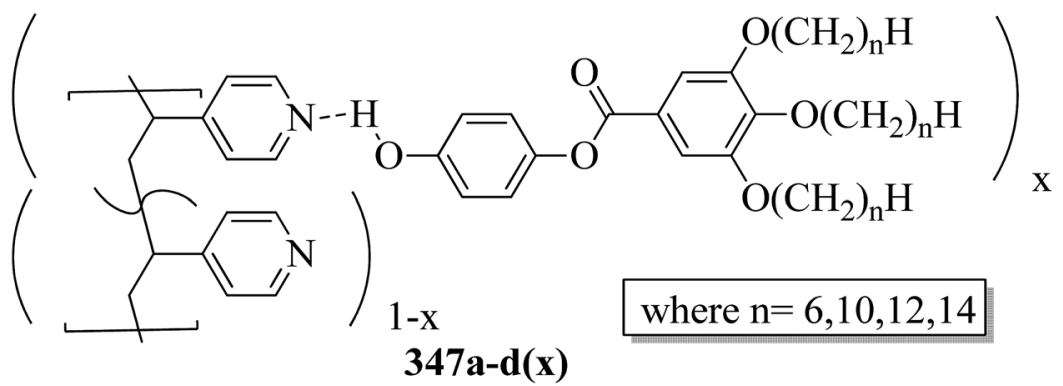

Fig. 23 Hydrogen bonded complexes 347a-d(x). 


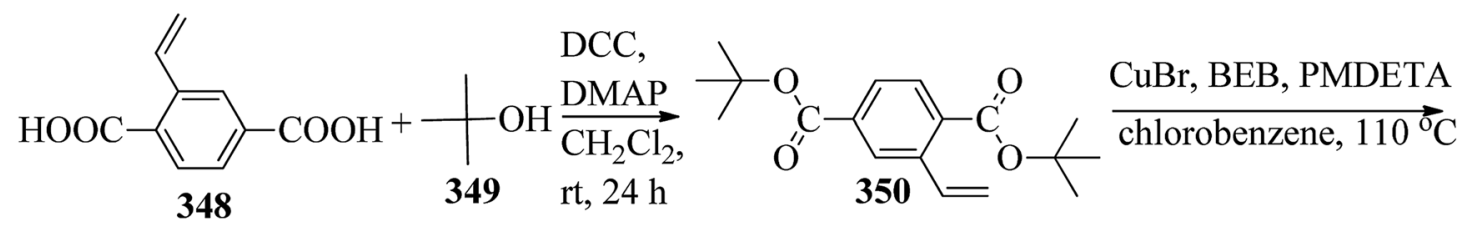

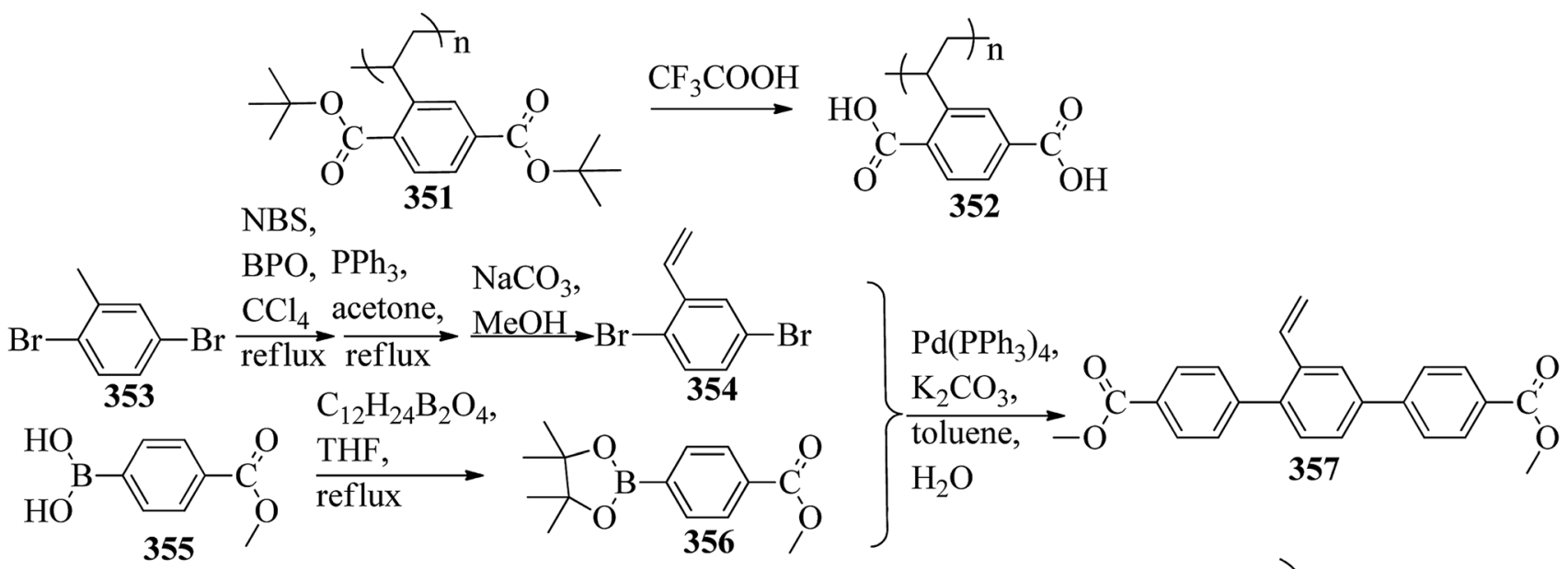<smiles>C=Cc1cc(-c2ccc(C(=O)OC(C)(C)C)cc2)ccc1-c1ccc(C(=O)OC(C)(C)CC(C)C)cc1</smiles>

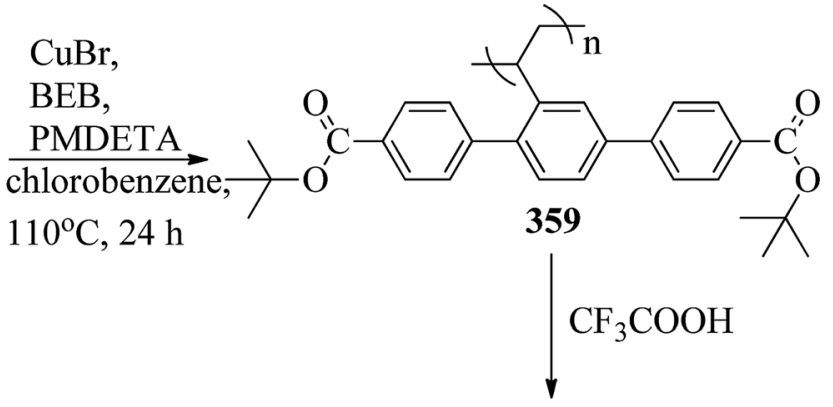

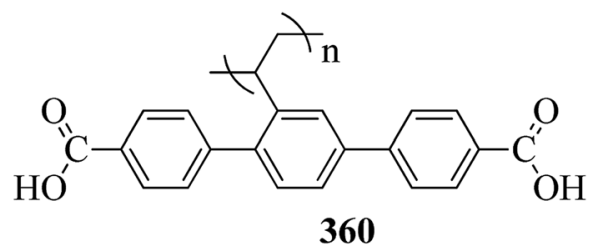

Scheme 44 Synthetic route for the polymers 352 and 360 .

tridecyloxybenzoyloxy)- $4^{\prime}$-stilbazole (210c), were synthesized by referring to the report by Lee et al. $;^{89}$ then, the hydrogen-bonded discotic complexes were prepared by mixing the stilbazole derivatives with 209 in THF solvent, and the solvent was removed slowly under reduced pressure (Scheme 28). Molecule 209 was non-mesogenic; among the three stilbazole derivatives, 210a exhibited smectic phase, while the others were nonmesogens. The phase transitions of the synthesized discotic complexes (211a-c) were found to be different from those of 210a-c. High enthalpy changes and monotropic phases were observed in complexes 211a and 211b; however, in the case of 211c, enantiotropic phase was observed. A small enthalpy change was observed in the case of complex 211c because the number of flexible alkyl chains decreased the $\pi-\pi$ interactions of stilbazole arms in the discotic mesogen. Complex 211a exhibited rectangular columnar phase, whereas complexes 211b and 211c exhibited hexagonal columnar phases. Along the column axes of these complexes, dipole-dipole interactions occur between the carbonyl groups; hence, they exhibited rectangular columnar and hexagonal columnar mesophases. These derivatives may be useful in electronic applications.

Coelho et al. ${ }^{90}$ synthesized seven new bent-shaped and polycatenar bent-shaped compounds which are derivatives of chalcone and cyanopyridine. Using POM, DSC, and XRD, the mesomorphic behavior of the compounds was investigated and correlated with their molecular structures. First, substituted benzoic acids were synthesized by referring to the previous report by Dal Bó et al. ${ }^{91}$ Further, the acid group was protected by Fischer esterification, followed by alkylation of the hydroxyl group (Williamson etherification) using 1-bromododecane, butanone and $\mathrm{K}_{2} \mathrm{CO}_{3}$. Also, TBAB (tetrabutylammonium bromide) was used to ensure complete alkylation in cases of 
alkylation of more than one hydroxyl group. Finally, the ester-toacid conversion was performed through base hydrolysis. At the same time, chalcone $\mathbf{2 1 2}$ was prepared through a ClaisenSchmidt condensation reaction of 4-hydroxybenzaldehyde and 4-hydroxyacetophenone in the presence of boron trifluoride diethyl etherate in dioxane. ${ }^{\mathbf{9 2}}$ Further, to obtain the cyanopyridine centre (213), chalcone was cyclised using sodium methoxide and malononitrile in methanol. ${ }^{93}$ Finally, the substituted carboxylic acids were converted to their respective benzoyl chlorides using $\mathrm{SOCl}_{2}$; then, they were esterified with the dihydroxyl bent core $\mathbf{2 1 2}$ or $\mathbf{2 1 3}$ (Scheme 29). Only two bent-core hexacatenar molecules (215c and 216c) exhibited liquid crystal properties at room temperature, and all the other molecules (215a-b and 216a-b,d) were non-mesogens. POM studies revealed that compound $215 \mathrm{c}$ exhibited monotropic liquid crystalline behavior, i.e. it exhibited a fan-shaped focal conic texture (hexagonal columnar) on cooling, whereas 216c exhibited enantiotropic liquid crystalline behavior, i.e. it exhibited the fan-shaped focal conic texture (hexagonal columnar) on cooling as well as on heating. Also, it was revealed that as the number of the alkoxy chains increased, the melting points of compounds 215a-b, 216a-b, and 216d and the clearing temperatures of 215c and 216c decreased. This is because the molecular packing was hindered by the additional alkoxy chains. Further, it was noted that chalcone core derivatives 215a-c had higher transition temperatures than cyanopyridine core derivatives 216a-d, even though 216a-d contain one extra ring in their structures, because of the decrease in the co-planarity between the benzene ring and the pyridine heterocycle due to steric effects. The cyanopyridine derivatives showed average luminescence quantum yields (i.e. ranging between $18 \%$ and $27 \%$ ) with an emission maximum at $371 \mathrm{~nm}$. Fluorescence was induced in the target molecules because of the inclusion of the cyanopyridine core between $\pi$-conjugated structures. This study also indicated that the length and position of the alkyl chains does not influence the luminescence properties of these compounds.

Lee $^{94}$ prepared hydrogen bonded supramolecular discotic liquid crystals using mesogenic stilbazole derivatives (218a-c) and non-mesogenic 1,3,5-tris(4-hydroxyphenyl)benzene (217). The three stilbazole derivatives $(\mathbf{2 1 8 a}-\mathbf{c})$ were synthesized according to previous literature, i.e. Lee et al. ${ }^{89}$ The discotic complexes were prepared by dissolving stilbazole derivatives 218a-c and 217 in anhydrous acetone and sonicating for $1 \mathrm{~h}$; then, the solvents were evaporated under reduced pressure (Scheme 30). The prepared discotic complexes (219a-c) exhibited monotropic mesophases by quenching the enantiotropic smectic mesophase behavior of the stilbazole derivatives; also, the prepared complexes showed lower mesophase transition temperatures than the stilbazole derivatives. Nematic columnar mesophases were observed in complexes 219a and 218b, whereas discotic nematic mesophase was observed in complex 219c. This is because of the long alkyl chains attached to the central core, which decrease the packing efficiency of the asymmetric discotic core.

Pfletscher et al. ${ }^{95}$ prepared a series of C3-symmetric assemblies by combining simple non-mesogenic azopyridines and commercially available core units (cyanuric acid (221), trimesic acid (220) and phloroglucinol (222)) and studied their liquid

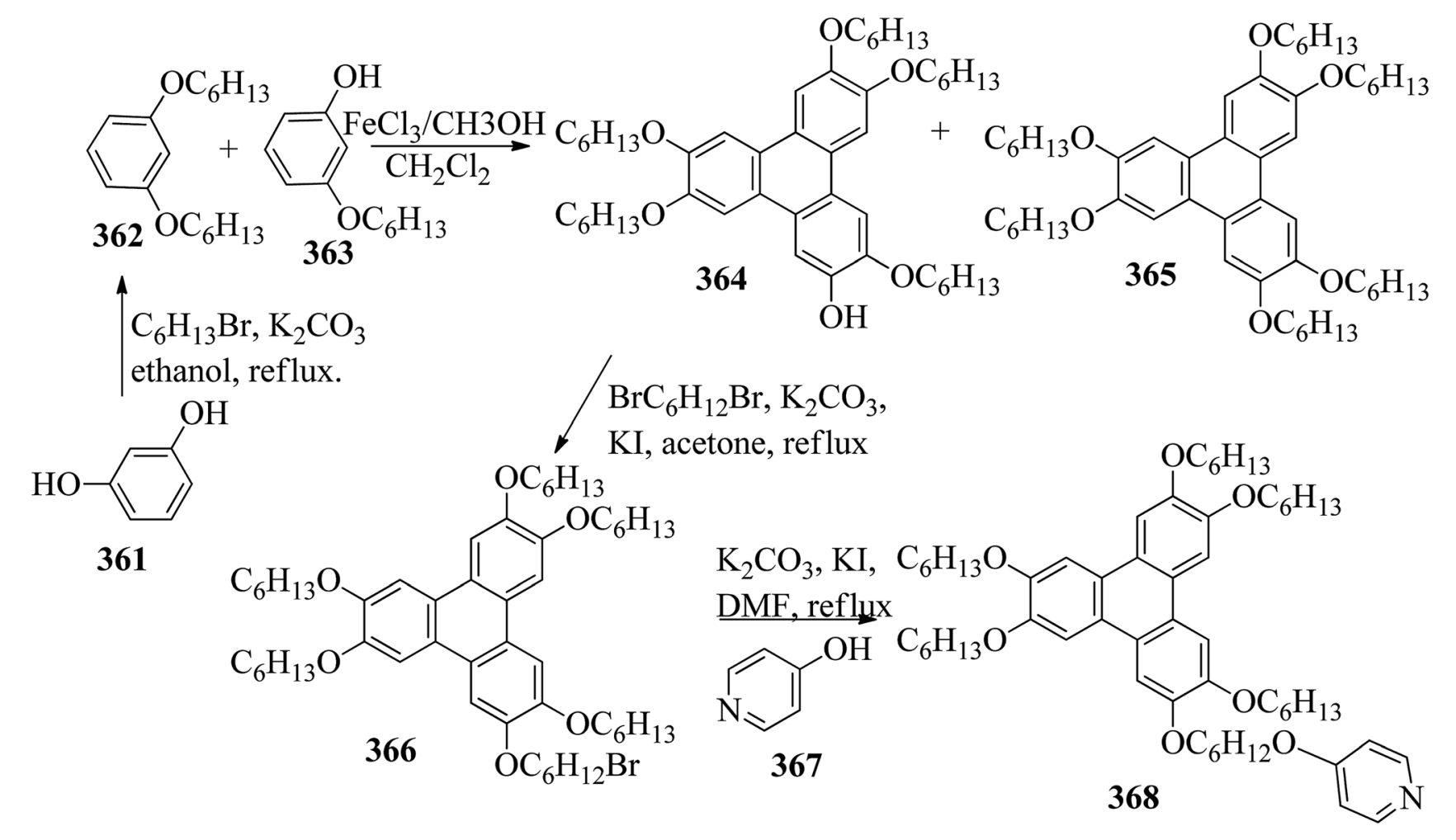

Scheme 45 Synthetic route for compound 368. 


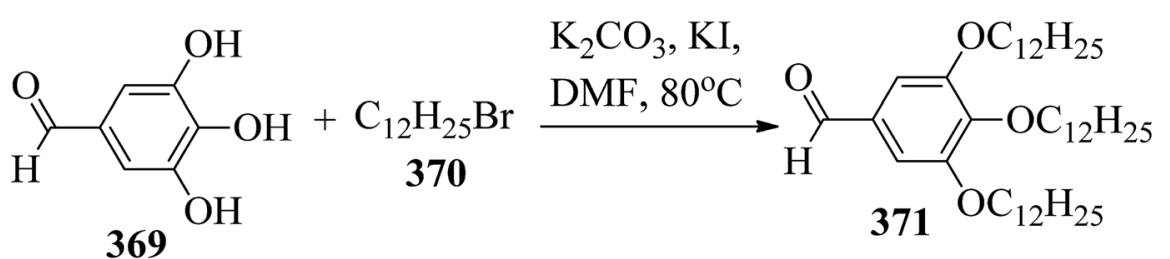

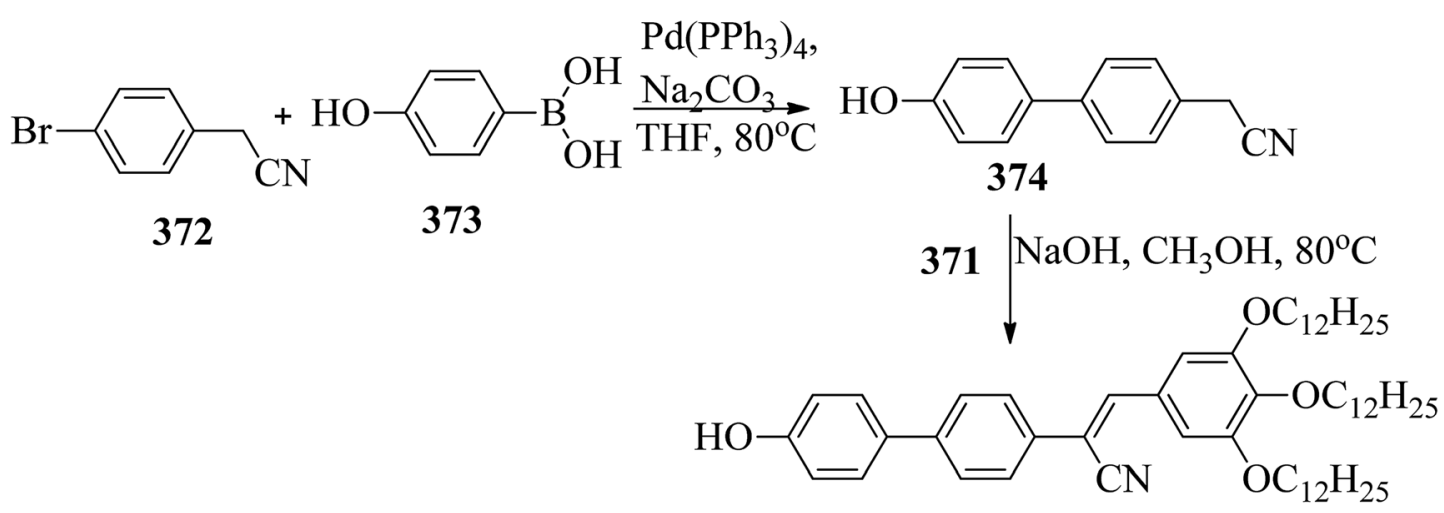

375

Scheme 46 Synthesis of 375 .

crystalline properties as well as their fast and reversible responses to irradiation with light. The commercially available core units and azopyridine derivatives (223a-h) were dissolved in acetone solvent, followed by heating in a microwave oven; then, slow evaporation of the solvents afforded the final expected complexes. The structures of proton donors 220, 221, and $\mathbf{2 2 2}$ and proton acceptors 223a-h are presented in Fig. 13. All the individual starting materials were non-mesogenic in nature. However, selected core units displayed C3-symmetry and common potential to form clusters with the azopyridine moieties; great variations were noted in the mesomorphic behavior of the assemblies. The authors expected weaker interactions in the hydrogen bonded phloroglucinol complexes and in the hydrogen bonded trimesic and cyanuric acid complexes. However, they observed inhomogeneous melting in the hydrogen bonded trimesic and cyanuric acid complexes at the time of the POM study. In contrast, inhomogeneous melting formed isotropic phase in the hydrogen bonded phloroglucinol

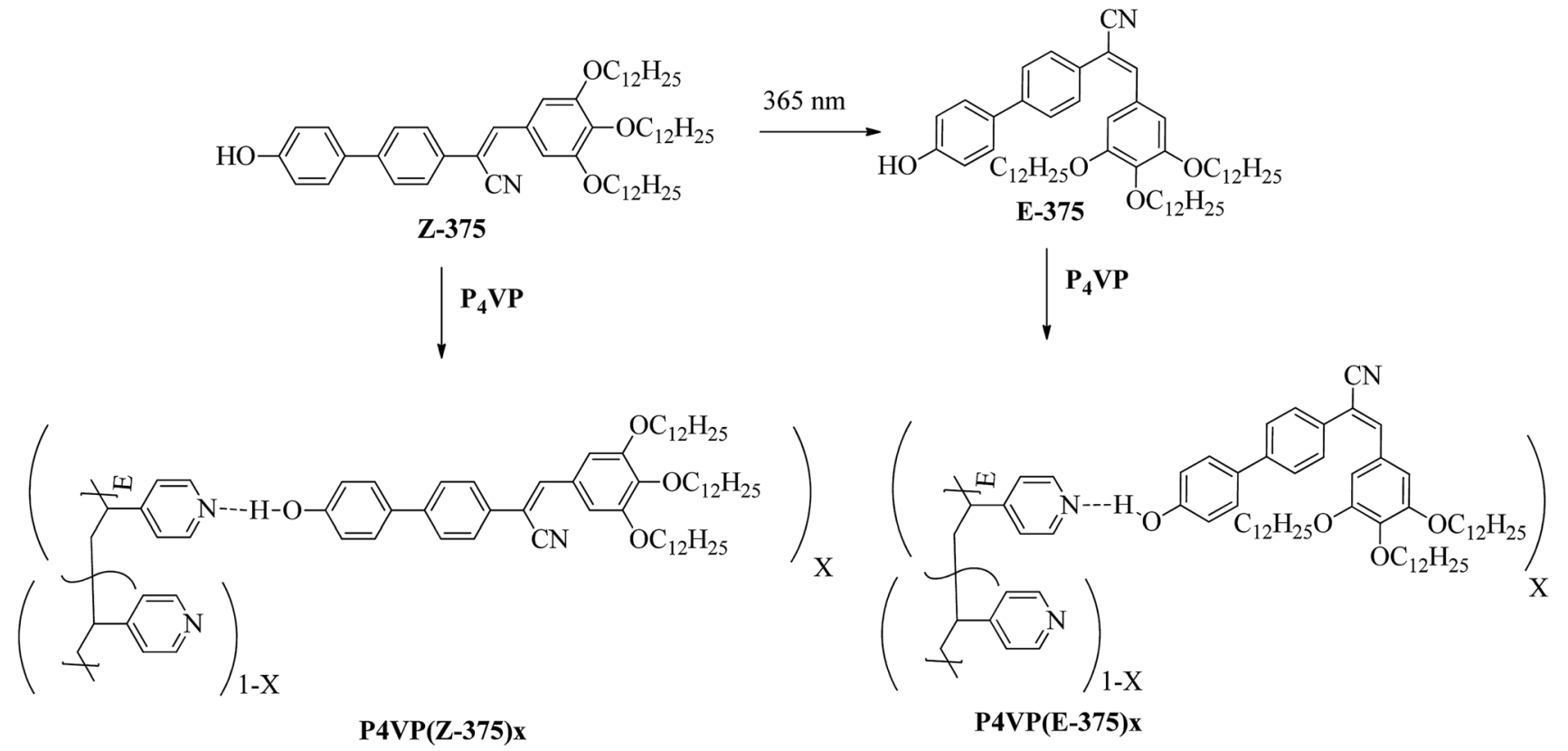

Scheme 47 Synthesis of P4VP(Z-375)x and P4VP(E-375)x. 

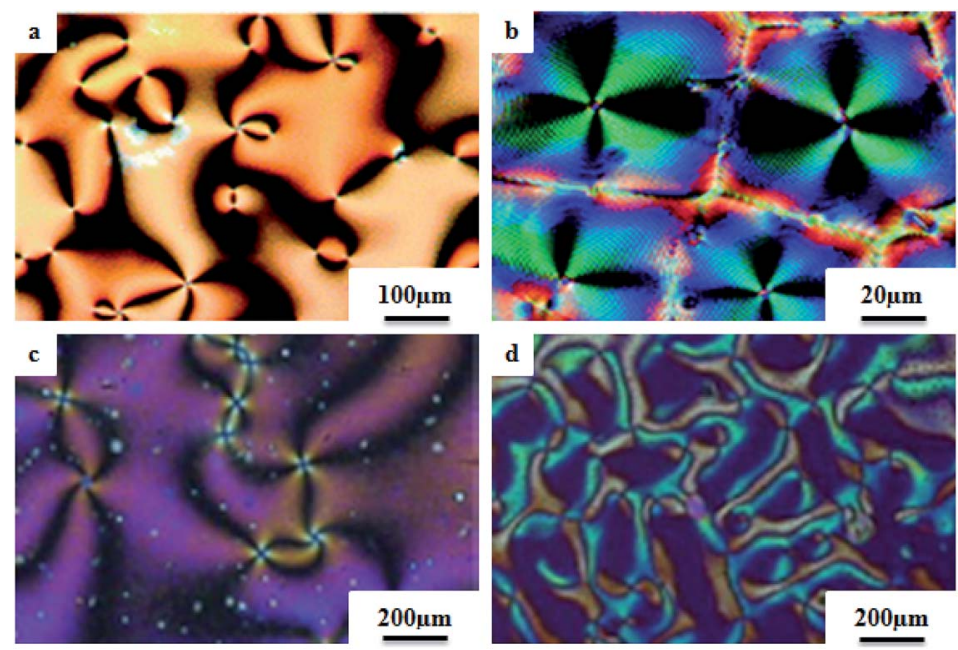

Fig. 24 Textures of (a) nematic, (b) nematic*, (c) discotic nematic, and (d) columnar nematic phases observed by POM. ${ }^{94,123}$

complexes. This finding was ascribed to the disaggregation of the hydrogen bonded complexes of cyanuric and trimesic acid clusters with azopyridine in favour of powerful hydrogen bonding within the phase-partitioned homo-crystals of trimesic and cyanuric acid. This is in line with the notably higher melting points of cyanuric acid $\left(320^{\circ} \mathrm{C}\right)$ and trimesic acid $(380$ $\left.{ }^{\circ} \mathrm{C}\right)$ with respect to phloroglucinol $\left(215^{\circ} \mathrm{C}\right)$. During the cooling cycle of the phloroglucinol series, they observed Schlieren textures (characteristic of nematic mesophase). Hence, they focused only on the phloroglucinol series. In the phloroglucinol series, 222/223a was found to be non-mesogenic in nature, i.e. it melted isotropically at $142{ }^{\circ} \mathrm{C}$. Upon comparing the thermal properties, they observed that increasing the alkyl chain length at the peripheral unit stabilizes the mesophases. $\Delta T$ varied from $5.6{ }^{\circ} \mathrm{C}$ for $\mathbf{2 2 2} / \mathbf{2 2 3 b}$ to $26.7{ }^{\circ} \mathrm{C}$ for $222 / 223 g$. In addition, the samples were illuminated with a laser pointer $(405 \mathrm{~nm}, 5 \mathrm{~mW})$ to study the photo-responsive properties of the hydrogen bonded liquid crystals. The same effect was observed for all the complexes. Originally, the complexes were in the trans state, which shows the Schlieren texture of nematic phase. However, the liquid crystal texture disappeared on illumination at $405 \mathrm{~nm}$, which provides evidence for photo-induced phase transition from mesophase to isotropic phase. The phase shift was ascribed to the photo-isomerisation of the azopyridine units from the trans to cis configuration because the bent shape of the cis isomers leads to mesophase destabilization. Mesophase was observed within a second by cutting off the irradiation. The sample was illuminated with a red $(650 \mathrm{~nm}, 5 \mathrm{~mW})$ and a green $(532 \mathrm{~nm}, 5 \mathrm{~W})$ laser pointer to repeat the cis/trans isomerization of the azopyridine and thereby cause the photoinduced phase transition. No effect on the mesophases was noted. Hence, they proposed that the formerly noted phase

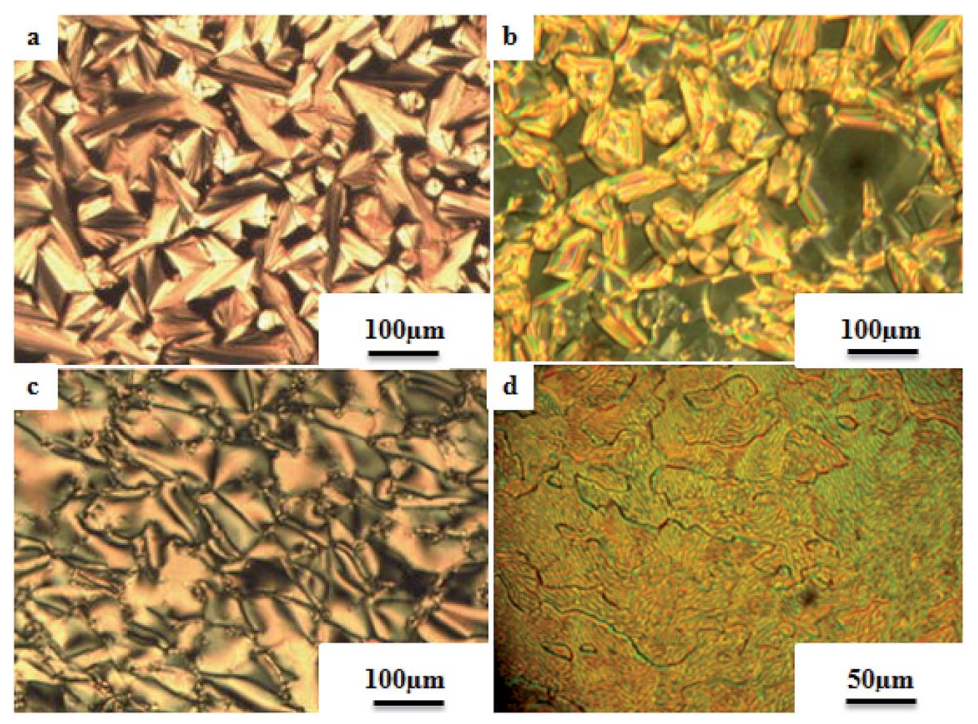

Fig. 25 Textures of (a) smectic A, (b) smectic B, (c) smectic C, and (d) smectic X phases observed by POM. ${ }^{61,124,125}$ 

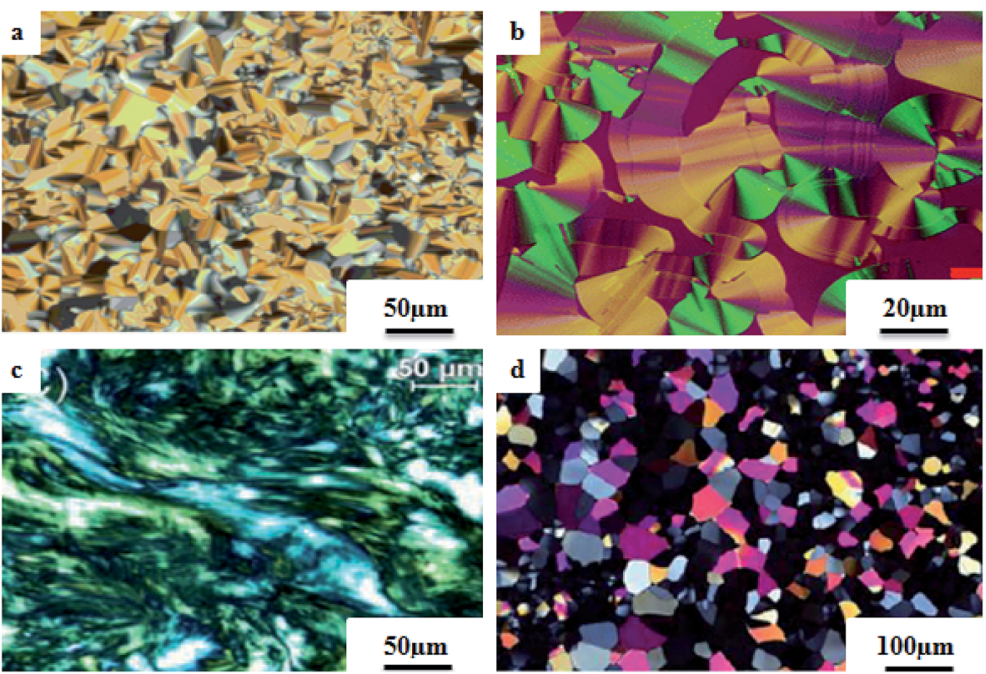

Fig. 26 Textures of (a) columnar hexagonal, (b) columnar rectangular, (c) columnar orthorhombic, and (d) oblique columnar phases observed by POM. $90,97,126,127$

transition was due to azopyridine moiety isomerisation and not to local thermal excitation. Due to these properties of the complexes, they are attractive for opto-electronic applications.

Spengler et al. ${ }^{96}$ prepared hydrogen bonded complexes using phloroglucinol (224) and its mono-fluorinated derivative (225) as the hydrogen bond donors and a series of azopyridyl side chains with different fluorination patterns as the hydrogen bond acceptors. The core unit and azopyridyl (Ap) side chain were taken in 1:3 molar ratios and separately dissolved in acetone solvent followed by mixing of the solutions. After solvent evaporation, the clusters were dried under vacuum. Fig. 14 presents the structures of the core units and azopyridyl side chains. None of the individual starting materials exhibited liquid crystal properties. In contrast, most of the prepared hydrogen bonded complexes exhibited mesogenic properties on cooling. In addition, they used the 224/226a-f series as a reference to compare their mesomorphic behavior with that of the fluorinated complexes. The complexes of 226a-f and 227a-f with 224 as well as with 225 exhibited the characteristic Schlieren appearance of a nematic mesophase; the complexes of 228a-f with 224 or $\mathbf{2 2 5}$ did not exhibit mesophase, i.e. they crystallized directly from isotropic liquid phase. Similar behavior was noted for the complexes of 229a-f with 225 . However, the typical conic texture of smectic phase was observed in the complexes of 229a-f with 224. The mesophase range moderately broadened from $\Delta T=8.9^{\circ} \mathrm{C}$ for $224 / 226 a$ to $\Delta T=21.0^{\circ} \mathrm{C}$ for $\mathbf{2 2 4} / \mathbf{2 2 6} \mathrm{c}$. Further increasing the alkyl chain length caused the mesophase ranges to decrease. A slight evenodd effect was observed on the transition temperatures. Similar morphologies were observed in the 225/226a-f aggregates when compared to the 224/226a-f aggregates. However, fluorination on the core unit affects the transition temperature. While $T_{\mathrm{c}}$ was
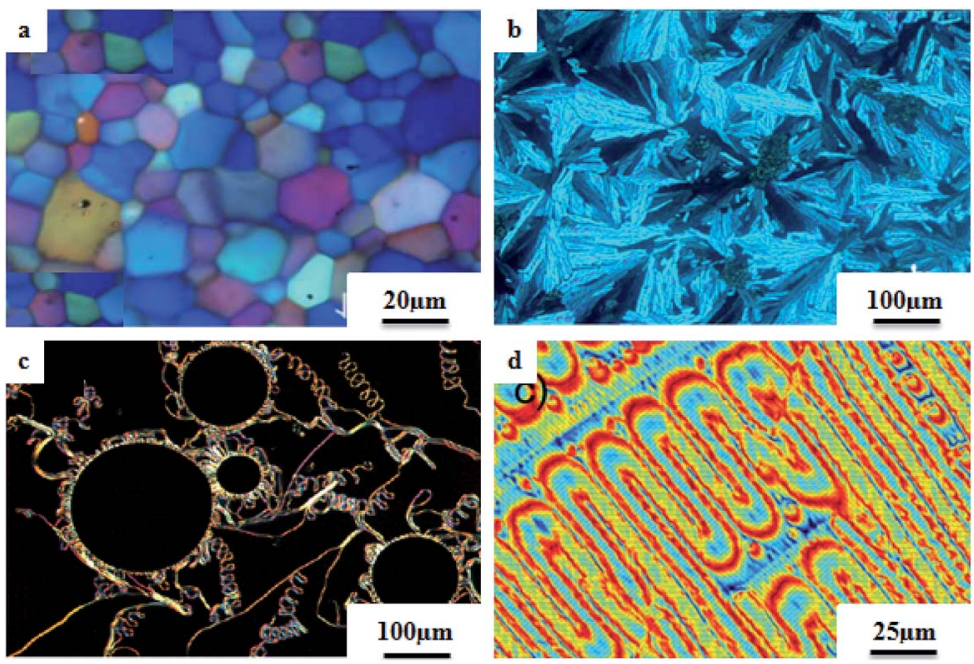

Fig. 27 Textures of (a) BP*, (b) B1, (c) TGBA*, and (d) twist bend nematic phases observed by POM. ${ }^{128-131}$ 
unaffected, $T_{\mathrm{N} \text {-Cr }}$ was notably lowered, leading to moderate widening of the mesophase ranges. The widening of the mesophase was ascribed to the stabilization of the hydrogen bonded complexes by non-classical hydrogen bonds between the Ap side chains (C-F $\cdots \mathrm{H}-\mathrm{C}$ ) and the core unit. 224/228a-f and $\mathbf{2 2 5} / \mathbf{2 2 8 a}-\mathbf{f}$ did not exhibit mesophases. However, shifting of their melting points towards lower temperatures was observed with respect to the non-fluorinated Ap analogues. This is because repulsive interactions between the electronic environment of the diazo bond and the fluoro substituent decrease the co-planarity of the Ap system and, as a result, the stability of the mesophase. 224/227a-f and 225/227a-f displayed liquid crystal behavior, in contrast to $224 / 228 a-f$ and $237 / 240 a-f$. By increasing the alkyl chain length in the 224/227a-f complexes, the mesophase range was found to increase from $\Delta T_{\text {meso }}=$ $8.1{ }^{\circ} \mathrm{C}(n=6)$ to $\Delta T_{\text {meso }}=13.3^{\circ} \mathrm{C}(n=12)$. Up to a chain length of $n=9$, the mesophase ranges of the 224/226a-f complexes increased and then gradually decreased again. $T_{\mathrm{c}}$ of the 224/ 227a-f complexes notably decreased compared to those of the 224/226a-f complexes, which is a well-known effect of lateral fluoro substitution. Different mesomorphic behavior was observed in the 224/229a-f and 225/226a-f complexes than in previously described systems, i.e. they exhibited characteristic textures of smectic phase, while nematic phases were observed in all other systems. Illumination by a laser pointer (405 nm, 5 $\mathrm{mA}$ ) of the complexes resulted in photoinduced phase transition from mesophase to isotropic phase. In the complex 224/ $227 \mathrm{~b}, \mathrm{~N}-\mathrm{I}$ switching occurred instantaneously ( 1 to $2 \mathrm{~s}$ ); after the laser was turned off, the complex required around $\sim 8 \mathrm{~s}$ to return to its original state. Longer times ( $\sim 3$ to $5 \mathrm{~s}$ ) were observed in 224/229b for the mesophase to isotropic phase transition and also for the reverse process ( $\sim 12 \mathrm{~s})$. The remarkably longer response of the difluorinated compound can be ascribed to the high molecular order of the smectic phase.

Vinayakumar ${ }^{97}$ synthesized mesogenic bird-shaped cyanopyridone derivatives and studied their mesogenic properties. The synthetic route for the preparation of the cyanopyridonebased mesogens is illustrated in scheme 31. Williamson etherification of catechol $\mathbf{2 3 0}$ followed by Friedel-Crafts acylation with acetyl chloride yielded 3,4-dialkoxyacetophenones as intermediates (232a-d). Alkylation of 3,4-dihydroxybenzaldehyde 234 using $N$-alkyl bromides yielded the other intermediates, 3,4-dialkoxybenzaldehydes 233a-d. Symmetrically substituted compounds $\mathbf{2 3 6 a - d}$ were synthesized by reacting intermediates $\mathbf{2 3 3 a - d}$ with precursors such as $232 a-$ d in the presence of excess ammonium acetate and ethyl cyanoacetate. Using different synthetic protocols, they separately synthesized the 2-methoxy-3-cyanopyridine derivatives 237a-c. Here, compound 237a was prepared by Claisen-Schmidt condensation of ketone 232a with aldehyde 233a to yield the chalcone, followed by chalcone cyclization with malononitrile in the presence of sodium methoxide. In this method, they obtained poor yield of the product; hence, they used an alternate method to obtain 237a. In this method, using methyl iodide, they alkylated compound 236a directly in the presence of base to obtain compound 237a in good yield. Using a similar process, they prepared the other two compounds, 237b and 237c. The thermotropic mesophase action of 236a-d showed that increasing the peripheral chain length led to destabilization of their mesogenic properties. Columnar oblique phase was observed in 236a with four hexyloxy chains. In contrast, two distinct columnar hexagonal mesophases were observed as the chain length in $\mathbf{2 3 6 b}$ increased by two methylene groups, which may be due to the increase in the diameter of the overall disc. According to the order of molecules along the columnar axis, dodecyloxy-substituted compounds 236b and 236c displayed intra-columnar transitions from ordered hexagonal columnar phases to disordered hexagonal columnar phases. Hence, in this type of mesogen, the symmetry of the columnar phases is decided by the chain length. Changes in enthalpy of about $4.06 \mathrm{~kJ} \mathrm{~mol}^{-1}$ and $2.49 \mathrm{~kJ} \mathrm{~mol}^{-1}$ were observed for the transition from ordered to disordered columnar phases in the cases of 236b and 236c, respectively. For the weaker columnar transitions, they noted higher values of enthalpy, which was ascribed to dipolar interactions as well as the strong intermolecular hydrogen bonding possessed by the mesocompounds in oblique hexagonal columnar phase. Thus, significant energy is required to disturb the order of the molecules. Hence, this intracolumnar transition was assigned as the first order transition. The XRD results revealed that the columnar mesophases in compounds 236a-d comprise two molecules per slice of column. Therefore, they presumed that the central cyanopyridone core participates in dimer formation through intermolecular hydrogen bonding to afford the disc-shaped structures. They synthesized another series of molecules in order to prove this assumption, 237a-c, by replacing the central core cyanopyridone with 2-methoxy-3-cyanopyridine, which lacks hydrogen bonding sites. However, the compounds failed to exhibit any mesomorphic character, which supports that the part of the central core forms the dimer arrangement. Finally, they concluded that mesophase formation in the bird-shaped cyanopyridones was decided by hydrogen bonding interactions.

Recently, Wang et al. ${ }^{98}$ synthesized a series of star-shaped mesogens comprising a $\left[1,1^{\prime}\right.$-biphenyl $]-4,4^{\prime}$-diyl diisonicotinate moiety carrying different counter ions, such as $\mathrm{Br}^{-}$, dodecylbenzenesulphonate anion, (+)-10-camphorsulfonate anion, $\mathrm{H}_{2} \mathrm{PO}_{4}{ }^{-}$and $\mathrm{BF}_{4}{ }^{-}$. Further, these compounds exhibited nematic phase, and it was noted that the mesophase temperature range increased with increasing alkyl chain length and that the clearing point decreased with increasing anion size. These starshaped liquid crystals displayed good ionic conductivity and properties, which was further attributed to electrostatic attraction of ions, electron delocalization of the $\pi-\pi$ stacking conjugation effect and the long and ordered molecular structure.

\subsection{Pyridine-based bent-shaped mesogens}

In bent-shaped mesogens, the two mesogenic groups are connected through a bent-shaped rigid core, e.g., resorcinol or isophthalic acid. In this section, the synthetic routes available for the preparation of different pyridine-based bent-shaped mesogens and their properties are summarized. 
Ahmed and Naoum $^{99}$ prepared new hydrogen bonded supramolecular complexes using 1 : 1 molar ratios of 4-(3'-pyridylazo)-4"-alkoxybenzoates (239a-f) and 4-alkoxyphenylazo benzoic acids (238a-f) with terminal alkoxy chain lengths varying from 6 to 16 carbons; they studied their mesophase behavior by DSC and polarizing light microscopy (PLM). Pyridine-based derivatives 239a-f and 4- $n$-alkoxyphenylazo benzoic acids 238a-f were prepared as described in previous work by Naoum et al.,$^{100} \mathrm{Janietz}$ et al. ${ }^{42}$ and Lizu et al. ${ }^{101}$ The

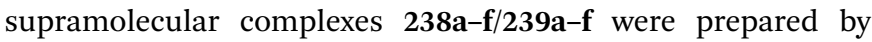
melting of 238a-f and 239a-f followed by stirring and drying (Scheme 32). All the complexes exhibited smectic $\mathrm{C}$ and nematic mesophases. The formation of hydrogen bonds was confirmed by FT-IR and UV-visible absorption spectroscopy. This study revealed that increasing the length of the alkoxy chain decreases the nematic transition enhancement $(\Delta T)$ in the case of the base component; on the other hand, $\Delta T$ increases with increasing chain length in the acid component, so the nematic phase stability is dependent on the alkoxy chain length of the acid component. The entropy associated with the smectic transition $\left(\Delta S_{\mathrm{C}-\mathrm{N}}\right)$ is greater than that associated with the nematic transition $\left(\Delta S_{\mathrm{N}-\mathrm{I}}\right)$. The entropies of both types vary unevenly with both alkoxy chain lengths $m$ and $n$. Due to the nonlinear shape of the supramolecular complexes, a low entropy change occurs. The stability of the mesophases (TC) of the prepared complexes (238a-f/239a-f) was found to be higher than that of their corresponding angular complexes prepared by Naoum et al.; ${ }^{100}$ however, smectic A phase was quenched and smectic $\mathrm{C}$ and nematic phases were formed for all complexes.

Han et al. ${ }^{102}$ prepared hydrogen bonded liquid crystal complexes with bent cores and compared them with their covalent analogues. Compounds 245 and 246 along with DCC and DMAP reagents were dissolved initially in dry DCM and maintained under nitrogen at room temperature for $16 \mathrm{~h}$. After completion of the reaction, the product was extracted using a water/DCM solvent mixture and the organic layer was dried using anhydrous magnesium sulphate. Further, the slow evaporation of solvent under reduced pressure afforded 247 as the product (Scheme 33). In a similar way, hydrogen acceptor 255 was synthesized using compound 253 and 3-hydroxypyridine (254) instead of compounds 245 and 246 (Scheme 34). Hydrogen donor 265 was synthesized by stirring compound 264 and $15 \%$ $\mathrm{Pd} / \mathrm{C}$ as a catalyst at room temperature in THF under hydrogen overnight. The catalysts were removed through Celite filtration, and the solvent was evaporated under reduced pressure to afford the product (Scheme 35). Further, appropriate molar ratios of hydrogen acceptors and hydrogen donors were dissolved in THF, and evaporating the solvents yielded the final hydrogen bonded complexes. The structures of the hydrogen bonded complexes, covalent bonded analogues, and hydrogen bonded dimers are represented in Fig. 15. Complexes 266/267 did not exhibit mesogenic properties, whereas complexes 255/ 265 and 255/270 showed nematic phases; also, their covalent analogue $\mathbf{2 4 7}$ was found to be non-mesogenic. This is because it has a more rigid bent core than the others. The hydrogen bonded diad 255/270 showed a higher phase transition temperature and narrower chiral nematic phase range than 255 /
Table 1 List of compounds which exhibit nematic phases and their temperature ranges

\begin{tabular}{|c|c|c|c|}
\hline Compound & Temperature range $\left({ }^{\circ} \mathrm{C}\right)$ & Phase & Ref. \\
\hline $8 \mathbf{a}$ & $131.0 \rightarrow 144.2$ & Nematic & 12 \\
\hline $8 \mathbf{b}$ & $90.6 \rightarrow 133.8$ & Nematic & \\
\hline $8 c$ & $95.7 \rightarrow 134.8$ & Nematic & \\
\hline $8 d$ & $87.2 \rightarrow 128.7$ & Nematic & \\
\hline $8 \mathrm{e}$ & $102.2 \rightarrow 137.1$ & Nematic & \\
\hline $8 f$ & $94.9 \rightarrow 133.9$ & Nematic & \\
\hline $8 g$ & $91.3 \rightarrow 130.2$ & Nematic & \\
\hline $15 a$ & $113.0 \rightarrow 120.0$ & Nematic* & 13 \\
\hline $15 b$ & $116.5 \rightarrow 136.5$ & Nematic* & \\
\hline $23 / 24 a$ & $125.0 \rightarrow 132.0$ & Nematic & 17 \\
\hline $23 / 24 b$ & $119.0 \rightarrow 158.0$ & Nematic & \\
\hline $29 a(4)$ & $154.0 \rightarrow 164.7$ & Nematic & 21 \\
\hline $29 a(6)$ & $111.5 \rightarrow 156.4$ & Nematic & \\
\hline $29 a(8)$ & $109.3 \rightarrow 149.1$ & Nematic & \\
\hline $29 a(10)$ & $127.6 \rightarrow 144.8$ & Nematic & \\
\hline $29 b(4)$ & $107.3 \rightarrow 115.4$ & Nematic & \\
\hline $29 b(6)$ & $110.9 \rightarrow 121.8$ & Nematic & \\
\hline $29 b(8)$ & $98.3 \rightarrow 114.4$ & Nematic & \\
\hline $29 b(10)$ & $96.9 \rightarrow 114.6$ & Nematic & \\
\hline $29 b(12)$ & $96.1 \rightarrow 104.1$ & Nematic & \\
\hline $40 \mathrm{~b}$ & $237.6 \rightarrow 257.2$ & Nematic & 22 \\
\hline 40c & $230.4 \rightarrow 245.7$ & Nematic & \\
\hline $38 \mathbf{b}$ & $203.0 \rightarrow 237.1$ & Nematic & \\
\hline $38 \mathrm{c}$ & $194.3 \rightarrow 226.3$ & Nematic & \\
\hline 38d & $196.2 \rightarrow 229.7$ & Nematic & \\
\hline $38 \mathrm{e}$ & $185.6 \rightarrow 219.2$ & Nematic & \\
\hline $38 f$ & $187.3 \rightarrow 220.5$ & Nematic & \\
\hline $38 \mathrm{~g}$ & $174.8 \rightarrow 215.6$ & Nematic & \\
\hline $38 \mathrm{~h}$ & $179.8 \rightarrow 220.1$ & Nematic & \\
\hline $57 b, d / 47 a$ & $186.9 \rightarrow 154.9$ & Nematic & 23 \\
\hline $57 c, d / 47 a$ & $168.7 \rightarrow 120.3$ & Nematic & \\
\hline $57 b, d / 47 a^{*}$ & $177.6 \rightarrow 123.8$ & Nematic* & \\
\hline $57 \mathrm{c}, \mathrm{d} / 47 \mathrm{a}^{*}$ & $157.5 \rightarrow 82.1$ & Nematic* & \\
\hline $57 b, d * / 47 a$ & $147.9 \rightarrow 126.3$ & Nematic* & \\
\hline $57 c, d^{*} / 47 a$ & $91.3 \rightarrow 73.5$ & Nematic* & \\
\hline $57 b, d * / 47 a^{*}$ & $84.9 \rightarrow 68.5$ & Nematic* & \\
\hline $57 \mathrm{c}, \mathrm{d}^{* / 47 a^{*}}$ & $64.3 \rightarrow 42.3$ & Nematic* & \\
\hline $79 \mathrm{c}$ & $112.7 \rightarrow 115.7$ & Nematic & 32 \\
\hline 79d & $104.9 \rightarrow 109.6$ & Nematic & \\
\hline $792 e$ & $81.7 \rightarrow 106.7$ & Nematic & \\
\hline $79 f$ & $82.3 \rightarrow 103.8$ & Nematic & \\
\hline $79 \mathrm{~g}$ & $93.5 \rightarrow 100.3$ & Nematic & \\
\hline $84 b / 85 b$ & $122.3 \rightarrow 117.7$ & Nematic & 37 \\
\hline $84 b / 85 f$ & $108.4 \rightarrow 106.7$ & Nematic* & \\
\hline $84 c / 85 c$ & $97.0 \rightarrow 57.3$ & Nematic & \\
\hline $84 d / 85 b$ & $61.4 \rightarrow 51.6$ & Nematic* & \\
\hline $84 d / 85 c$ & $88.6 \rightarrow 41.9$ & Nematic* & \\
\hline $\mathbf{8 4 d} / \mathbf{8 5 f}$ & $26.9 \rightarrow 13.9$ & Nematic* & \\
\hline $84 e / 85 c$ & $107.5 \rightarrow 76.3$ & Nematic & \\
\hline $84 f / 85 b$ & $132.8 \rightarrow 91.7$ & Nematic* & \\
\hline $84 f / 85 c$ & $140.1 \rightarrow 75.8$ & Nematic* & \\
\hline $84 f / 85 d$ & $78.3 \rightarrow 69.7$ & Nematic* & \\
\hline $84 f / 85 e$ & $91.3 \rightarrow 43$ & Nematic* & \\
\hline $84 f / 85 f$ & $111.1 \rightarrow 81.8$ & Nematic* & \\
\hline $89 a / 90 a$ & $134.4 \rightarrow 185.7$ & Nematic & 40 \\
\hline $89 a / 90 b$ & $135.0 \rightarrow 183.0$ & Nematic & \\
\hline $89 a / 90 c$ & $140.1 \rightarrow 190.7$ & Nematic & \\
\hline 89a/90d & $137.5 \rightarrow 180.0$ & Nematic & \\
\hline $89 b / 90 a$ & $143.8 \rightarrow 184.4$ & Nematic & \\
\hline $89 \mathrm{~b} / 90 \mathrm{~b}$ & $143.2 \rightarrow 180.1$ & Nematic & \\
\hline $89 b / 90 c$ & $136.7 \rightarrow 180.6$ & Nematic & \\
\hline $89 b / 90 d$ & $135.7 \rightarrow 173.2$ & Nematic & \\
\hline
\end{tabular}


Table 1 (Contd.)

Table 1 (Contd.)

\begin{tabular}{|c|c|c|c|c|c|c|c|}
\hline Compound & Temperature range $\left({ }^{\circ} \mathrm{C}\right)$ & Phase & Ref. & Compound & Temperature range $\left({ }^{\circ} \mathrm{C}\right)$ & Phase & Ref. \\
\hline $89 c / 90 a$ & $138.8 \rightarrow 204.2$ & Nematic & & $225 / 226 a$ & $92.3 \rightarrow 83.0$ & Nematic & \\
\hline $89 c / 90 b$ & $140.1 \rightarrow 196.3$ & Nematic & & $225 / 226 b$ & $90.7 \rightarrow 73.5$ & Nematic & \\
\hline $89 c / 90 d$ & $140.8 \rightarrow 193.2$ & Nematic & & $225 / 226 d$ & $91.0 \rightarrow 67.1$ & Nematic & \\
\hline $89 c / 90 a$ & $139.5 \rightarrow 194.4$ & Nematic & & $225 / 226 \mathrm{e}$ & $81.5 \rightarrow 69.7$ & Nematic & \\
\hline $89 c / 90 b$ & $138.2 \rightarrow 191.9$ & Nematic & & $225 / 226 f$ & $92.5 \rightarrow 82.1$ & Nematic & \\
\hline 109 & $80.7 \rightarrow 48.3$ & Nematic & 49 & $224 / 227 c$ & $77.3 \rightarrow 69.5$ & Nematic & \\
\hline 116 & $145.2 \rightarrow 239.9$ & Nematic & & $224 / 227 d$ & $80.0 \rightarrow 68.9$ & Nematic & \\
\hline $169 a$ & $50.3 \rightarrow 41.2$ & Nematic & 50 & $224 / 227 \mathrm{e}$ & $81.4 \rightarrow 69.6$ & Nematic & \\
\hline $169 b$ & $80.7 \rightarrow 48.3$ & Nematic & & $224 / 227 f$ & $84.8 \rightarrow 71.5$ & Nematic & \\
\hline $169 c$ & $63.7 \rightarrow 81.6$ & Nematic & & $225 / 227 a$ & $70.0 \rightarrow 65.4$ & Nematic & \\
\hline 169d & $77.1 \rightarrow 85.9$ & Nematic & & $225 / 227 b$ & $74.5 \rightarrow 63.6$ & Nematic & \\
\hline $177 a$ & $162.9 \rightarrow 236.0$ & Nematic & & $238 a / 239 a$ & $138.0 \rightarrow 241.2$ & Nematic & 93 \\
\hline $177 b$ & $160.9 \rightarrow 238.8$ & Nematic & & $238 a / 239 b$ & $138.1 \rightarrow 238.7$ & Nematic & \\
\hline $177 \mathrm{c}$ & $164.0 \rightarrow 236.7$ & Nematic & & $238 a / 239 c$ & $137.7 \rightarrow 238.5$ & Nematic & \\
\hline $177 d$ & $164.3 \rightarrow 238.0$ & Nematic & & $238 a / 239 d$ & $137.4 \rightarrow 238.5$ & Nematic & \\
\hline $126+50 \mathrm{wt} \% \mathbf{C L}$ & $119.0 \rightarrow 117.0$ & Nematic & 53 & $238 a / 239 e$ & $133.2 \rightarrow 238.0$ & Nematic & \\
\hline $152 a$ & $133.3 \rightarrow 186.3$ & Nematic & 63 & 238a/239f & $129.2 \rightarrow 238.3$ & Nematic & \\
\hline $152 b$ & $135.3 \rightarrow 156.0$ & Nematic & & $238 b / 239 a$ & $137.2 \rightarrow 202.6$ & Nematic & \\
\hline $152 \mathrm{c}$ & $116.7 \rightarrow 149.4$ & Nematic & & $238 b / 239 b$ & $140.8 \rightarrow 200.1$ & Nematic & \\
\hline 152d & $131.0 \rightarrow 144.9$ & Nematic & & $238 b / 239 c$ & $137.8 \rightarrow 201.9$ & Nematic & \\
\hline $152 e$ & $125.3 \rightarrow 139.4$ & Nematic & & $238 b / 239 d$ & $137.2 \rightarrow 202.0$ & Nematic & \\
\hline $162 a$ & $128.0 \rightarrow 141.0$ & Nematic & 70 & $238 b / 239 e$ & $137.0 \rightarrow 201.0$ & Nematic & \\
\hline $162 b$ & $134.0 \rightarrow 139.0$ & Nematic & & $238 b / 239 f$ & $137.0 \rightarrow 198.7$ & Nematic & \\
\hline $162 c / 163 b$ & $109.0 \rightarrow 188.0$ & Nematic & & $238 d / 239 c$ & $137.1 \rightarrow 167.3$ & Nematic & \\
\hline $162 d / 163 b$ & $110.0 \rightarrow 189.0$ & Nematic & & $238 d / 239 d$ & $137.7 \rightarrow 168.3$ & Nematic & \\
\hline $186 a$ & $146.8 \rightarrow 158.9$ & Nematic & 75 & $238 d / 239 e$ & $138.6 \rightarrow 167.9$ & Nematic & \\
\hline $186 b$ & $126.1 \rightarrow 152.3$ & Nematic & & $238 d / 239 f$ & $138.2 \rightarrow 168.2$ & Nematic & \\
\hline $187 a$ & $86.2 \rightarrow 109.4$ & Twist bend nematic & & $238 \mathrm{e} / 239 a$ & $139.3 \rightarrow 153.1$ & Nematic & \\
\hline $187 a$ & $109.4 \rightarrow 166.4$ & Nematic & & $238 \mathrm{e} / 239 \mathrm{~b}$ & $139.8 \rightarrow 154.6$ & Nematic & \\
\hline $187 \mathrm{~b}$ & $93.5 \rightarrow 98.0$ & Twist bend nematic & & $238 \mathrm{e} / 239 \mathrm{c}$ & $140.2 \rightarrow 153.8$ & Nematic & \\
\hline $187 \mathrm{~b}$ & $98.0 \rightarrow 157.7$ & Nematic & & $238 \mathrm{e} / 239 d$ & $140.0 \rightarrow 154.7$ & Nematic & \\
\hline $192 a$ & $86.0 \rightarrow 77.0$ & Nematic & 77 & $238 \mathrm{e} / 239 \mathrm{e}$ & $140.1 \rightarrow 154.5$ & Nematic & \\
\hline $196 a$ & $115.7 \rightarrow 128.3$ & Nematic & 80 & $238 \mathrm{e} / 239 \mathrm{f}$ & $140.2 \rightarrow 154.6$ & Nematic & \\
\hline 196b & $109.6 \rightarrow 128.7$ & Nematic & & $238 f / 239 a$ & $137.7 \rightarrow 144.2$ & Nematic & \\
\hline $196 \mathrm{c}$ & $101.4 \rightarrow 125.3$ & Nematic & & $238 f / 239 b$ & $137.9 \rightarrow 145.9$ & Nematic & \\
\hline 196d & $117.9 \rightarrow 133.7$ & Nematic & & $238 f / 239 c$ & $137.3 \rightarrow 144.3$ & Nematic & \\
\hline $196 e$ & $109.0 \rightarrow 128.2$ & Nematic & & $238 f / 239 d$ & $138.4 \rightarrow 145.7$ & Nematic & \\
\hline $196 f$ & $122.2 \rightarrow 138.8$ & Nematic & & $238 f / 239 e$ & $138.0 \rightarrow 145.8$ & Nematic & \\
\hline $208 \mathrm{a}$ & $127.1 \rightarrow 142.8$ & Nematic & 82 & $238 f / 239 f$ & $138.5 \rightarrow 145.6$ & Nematic & \\
\hline 219a & $150.9 \rightarrow 120.0$ & Columnar nematic & 89 & $255 / 265$ & $92.7 \rightarrow 62.3$ & Nematic* & 96 \\
\hline $219 b$ & $152.8 \rightarrow 128.2$ & Columnar nematic & & $255 / 270$ & $101.3 \rightarrow 87.6$ & Nematic* & \\
\hline $219 c$ & $147.2 \rightarrow 103.4$ & Discotic nematic & & $278 / 288$ & $146.9 \rightarrow 96.6$ & Nematic* & 97 \\
\hline $224 / 226 a$ & $93.6 \rightarrow 84.7$ & Nematic & 91 & $278 / 286$ & $145.2 \rightarrow 88.1$ & Nematic* & \\
\hline $224 / 226 b$ & $91.4 \rightarrow 71.4$ & Nematic & & $277 / 285$ & $124.6 \rightarrow 97.4$ & Nematic* & \\
\hline $224 / 226 c$ & $91.1 \rightarrow 70.1$ & Nematic & & $277 / 284$ & $122.6 \rightarrow 97.4$ & Nematic* & \\
\hline $224 / 226 d$ & $91.9 \rightarrow 74.5$ & Nematic & & $278 / 285$ & $137.5 \rightarrow 106.6$ & Nematic* & \\
\hline $224 / 226 e$ & $92.9 \rightarrow 71.9$ & Nematic & & $278 / 284$ & $148.3 \rightarrow 86.8$ & Nematic* & \\
\hline $224 / 226 f$ & $91.9 \rightarrow 74.5$ & Nematic & & $276 / 287$ & $151.8 \rightarrow 111.2$ & Nematic* & \\
\hline
\end{tabular}


Table 1 (Contd.)

\begin{tabular}{llll}
\hline Compound & Temperature range $\left({ }^{\circ} \mathrm{C}\right)$ & Phase & Ref. \\
\hline 345a & $210.9 \rightarrow 260.1$ & Nematic & 108 \\
345b & $202.4 \rightarrow 251.7$ & Nematic & \\
345c & $208.3 \rightarrow 255.8$ & Nematic & \\
345d & $197.8 \rightarrow 245.9$ & Nematic & \\
345e & $201.1 \rightarrow 248.3$ & Nematic & \\
345f & $192.4 \rightarrow 238.4$ & Nematic & \\
346a & $207.3 \rightarrow 254.8$ & Nematic & \\
346b & $192.4 \rightarrow 238.4$ & Nematic & \\
346c & $203.0 \rightarrow 246.0$ & Nematic & \\
346d & $191.6 \rightarrow 224.4$ & Nematic & \\
346e & $194.0 \rightarrow 229.7$ & Nematic & \\
346f & $187.5 \rightarrow 218.0$ & Nematic &
\end{tabular}

265 with lateral fluoro-substitution. They also studied the effects of changing the molar ratio of the proton donor in the complexes. Complexes 255/265, 268/265, and 269/265 with a $1: 1$ molar ratio did not exhibit blue phases; however, blue phases started to form with increasing molar ratio of 265. Above $55 \mathrm{~mol} \%$, blue phase started to appear, but after $80 \mathrm{~mol} \%$, the blue phase disappeared; this is due to biaxial dilution of the over-supplied uniaxial rod-like dimer 265. Furthermore, they mixed the covalent analogue 247 with different molar ratios of 265 and checked for blue phases. Blue phase was induced at 75.0 to $82.4 \mathrm{~mol} \%$ of 265 . They also observed that increasing the alkyl chain length widens the blue phase range, i.e. 268/265 < $\mathbf{2 6 9} / \mathbf{2 6 5}<\mathbf{2 5 5} / \mathbf{2 6 5}$. Due to the narrow ranges of blue phase in the covalent-bonded bent-core mixtures, they concluded that introduction of hydrogen bonds in the bent-core effectively stabilizes the blue phase. The widest blue phase range of $\Delta T=$ $12.0{ }^{\circ} \mathrm{C}$ was observed in the $\mathbf{2 5 5} / 265\left(3 / 7 \mathrm{~mol} \mathrm{~mol}^{-1}\right)$ complex. To compare the effects of fluoro groups and the chiral centres of the $\mathrm{H}$ donors on the hydrogen bonded complexes, they prepared two more hybrid hydrogen bonded complexes with dual hydrogen donors, i.e. complex $255 /(265+271)$ and $255 /(265$ $+270)$. As the $271 \mathrm{~mol} \%$ in $255 /(265+271)$ complex increased, the blue phase range decreased and vanished above $70 \mathrm{~mol} \%$ of 271. In $255 /(265+270)$, as the 270 content increased, the blue phase range decreased faster than in the previous case; it vanished above $30 \mathrm{~mol} \%$ of $\mathbf{2 7 0}$ in the complex.

Han et al. ${ }^{103}$ synthesized a hydrogen bond acceptor and donors, prepared their complexes and studied their mesogenic properties. Compound 273 and pyridin-3-ol were dissolved in DCM, and DMAP was also added and dissolved under nitrogen; then, DCC was added to this solution, and the reaction was maintained for $16 \mathrm{~h}$ at room temperature. The reaction mixture was filtered and washed with DCM solvent, followed by extraction with deionized water/DCM; then, the organic phase was dried using anhydrous $\mathrm{MgSO}_{4}$ and concentrated using a rotary evaporator. Finally, the obtained product was purified by silica gel column chromatography to afford the pure hydrogen bond acceptor (276). Similarly, using compounds 274 and 275, the other hydrogen bond acceptors (277 and 278) were synthesized (Scheme 36). Compound 279 and benzyl 4-hydroxybenzoate were dissolved in DCM, and DMAP was also added and dissolved under nitrogen; further, DCC was added to the solution, and the reaction mixture was maintained for another $16 \mathrm{~h}$ at room temperature. The reaction mixture was filtered and washed with DCM solvent, followed by extraction with deionized water/DCM; then, the organic phase was dried using anhydrous $\mathrm{MgSO}_{4}$ and concentrated using a rotary evaporator. By silica gel column chromatography, the intermediate was collected. In $\mathrm{THF}$, the intermediate and $15 \% \mathrm{Pd} / \mathrm{C}$ were dissolved, and this reaction was maintained at room temperature overnight under hydrogen. After completion of the reaction, the mixture was filtered and washed with THF solvent. This was followed by solvent evaporation and recrystallizing in an $n$ hexane/DCM solvent mixture, which resulted in pure hydrogen donor 284. Similarly, other hydrogen donors (285288) were obtained using compounds 280-283 (Scheme 37). Finally, the hydrogen bonded complexes were prepared by dissolving appropriate amounts of proton acceptor and donor compounds in chloroform/THF ( $\sim 3: 1 \mathrm{vol})$ and evaporating the solvent slowly. The structures of hydrogen bonded complexes 289-295 and the structure of hydrogen bonded complex 296 are presented in Fig. 16. Only complex 293 exhibited a wide range of blue phases in addition to smectic A and cholesteric phases; all the other complexes exhibited smectic A and cholesteric phases only. Due to the more symmetric hydrogen bonded bent-core structure of 294 and its large helical twisting power (HTP), this complex exhibited lower phase transition temperatures and a wider cholesteric phase range than the supramolecular diad 295. Further, studying the effects of various numbers of the chiral centers on the mesogenic properties of complexes 292, 294 and 291 revealed that a wider cholestric phase $\left(\Delta T_{\mathrm{N}}^{*}=62.8^{\circ} \mathrm{C}\right)$ was observed for 294 than for the other two complexes. Therefore, they concluded that by introducing double chiral centers on both the proton acceptor and donor, the cholestric phase was stabilized. On the other hand, a narrower smectic A phase range of $\Delta T_{\mathrm{SmA}}=26.2^{\circ} \mathrm{C}$ was observed for complex 294 than for the other two complexes on cooling. Studies of the effects of the location of the chiral center on the mesogenic properties of complexes 292 and 291 revealed that the chiral center on side chain 291 has a narrower cholestric phase and smectic A phase than 292, where chirality was present on the flexible spacer. They also compared supramolecular diads with lateral fluoride substitution (i.e., 291, 292, and 294) with those without lateral fluoride substitution (i.e., 290, 293, 295) to study the effects of fluoride on the supramolecular diads. In the double chiral centred complexes, blue phase was observed in 293 but was not observed in 294. Smaller dipole moments and lower HTP values induced lower phase transition temperatures in all supramolecular diads without lateral fluoride substitution compared with the lateral fluoride substituted complexes. Due to the stronger helical twisting power HTP $=4.2 \mu \mathrm{m}^{-1}$ of 293 , blue phase was observed. They also studied the effects of the molar ratio of the proton acceptor/ proton donor on the blue phase of supramolecular diad 293; it was revealed that the widest blue phase range was observed at $50 \mathrm{~mol} \%$ of the hydrogen donor in 293 (i.e., proton acceptor/ proton donor $=1: 1$ ). This is due to the presence of rod-like 
and hydrogen bonded bent-core mesogens rather than when the hydrogen donor $=75$ mole $\%$ where the mesogen possesses a linear hydrogen bonded rod-like structure.

Trišović et al. ${ }^{\mathbf{1 0 4}}$ synthesized photoactive liquid crystals containing five-ring pyridine-based bent-cores with different substituents at the peripheral phenyl rings $\left(-\mathrm{OCH}_{3},-\mathrm{Cl}\right.$ and - $\mathrm{NO}_{2}$ ). 2,6-Bis[2-(4-(4-dodecyloxy-3-substituted benzoyloxy) phenyl)ethenyl]pyridines with different substituents, namely methoxy (306a), chloro (306b) and nitro (306c), in the $3^{\text {rd }}$ position of the peripheral phenyl were synthesized as shown in Scheme 38. 2,6-Bis[2-(4-hydroxyphenyl)ethenyl]pyridine (300) was prepared by referring to the procedure by Bergmann and Pinchas. ${ }^{105}$ 2,6-Lutidine (297) was refluxed with excess 4hydroxybenzaldehyde (298) and acetic anhydride to yield compound 299, of which base hydrolysis led to the formation of compound 300. 4-Dodecyloxy-3-substituted benzoic acids $(\mathbf{3 0 4 a}-\mathbf{c})$ were synthesized by referring to a previous report by Sivakumar et al., ${ }^{106}$ i.e. protection of the acid group by esterification and alkylation of the hydroxyl group using 1-bromododecane followed by deprotection of the acid group through base hydrolysis. Finally, the target molecules (306a-c) were synthesized by acylation of compound $\mathbf{3 0 0}$ with substituted benzoyl chlorides, i.e. 305a-c ( 2 equivalents) in the presence of triethylamine base. The liquid crystal properties of the synthesized compounds were studied using POM, DSC, and XRD and compared with those of the unsubstituted parent compound (Fig. 17), which was reported by Markovic et al. ${ }^{107}$ Studies revealed that on introducing the methoxy group, the mesogenic properties were quenched and the melting point decreased (110 ${ }^{\circ} \mathrm{C}$ ) compared to that of the parent compound. On the other hand, enantiotropic B1-like phase was observed in the chloroand nitro-substituted compounds. Chloro-substituted compound 306b exhibited a broader mesomorphic range than the parent compound 306p; however, the clearing point was found to be unchanged. On introducing strong electronwithdrawing nitro groups, they noted that the clearing temperature increased by $c a .15{ }^{\circ} \mathrm{C}$ and a somewhat narrower range of mesomorphic properties than the parent compound was obtained. The smectic phase formation suggests that these compounds exist in s-cis/s-cis conformation. Further, significant changes in the transition temperatures and textures of the mesophases were observed when the compounds were irradiated by UV light.

Pradhan et al. ${ }^{108}$ synthesized a bent-shaped molecule containing a pyridine core at the centre which was also substituted with thiadiazole or oxadiazole derivatives with different numbers and lengths of alkoxy chains. The expected molecules 311a-d and 312a-d and their precursors were prepared as illustrated in Scheme 39. O-Alkylation of the corresponding hydrazides (309a-d) was performed by referring to a previous report by Pradhan et al. ${ }^{109}$ 2,6-Di- $N$-pyridinebenzohydrazides 308a-d were synthesized by refluxing these hydrazides with 2,6pyridine dicarboxylic acid chloride and triethylamine base. Then, cyclization in the presence of phosphoryl chloride $\left(\mathrm{POCl}_{3}\right)$ yielded the dioxadiazole derivatives (311a-d) and cyclization in the presence of Lawesson's reagent in toluene yielded the dithiadiazole derivatives (312a-d). Among dioxadiazole
Table 2 List of compounds which exhibit smectic phases and their temperature ranges

\begin{tabular}{|c|c|c|c|}
\hline Compound & Temperature range $\left({ }^{\circ} \mathrm{C}\right)$ & Phase & Ref. \\
\hline $7 \mathbf{a}$ & $108.0 \rightarrow 136.2$ & Smectic A & 12 \\
\hline $7 \mathbf{b}$ & $103.3 \rightarrow 136.5$ & Smectic A & \\
\hline $7 \mathrm{c}$ & $94.8 \rightarrow 135.5$ & Smectic A & \\
\hline $7 d$ & $86.7 \rightarrow 139.5$ & Smectic A & \\
\hline $7 e$ & $90.2 \rightarrow 140.9$ & Smectic A & \\
\hline $7 f$ & $94.5 \rightarrow 143.6$ & Smectic A & \\
\hline $7 \mathrm{~g}$ & $91.3 \rightarrow 141.2$ & Smectic A & \\
\hline $15 \mathbf{a}$ & $42.7 \rightarrow 112.2$ & Smectic A & 13 \\
\hline $15 b$ & $74.2 \rightarrow 115.3$ & Smectic A & \\
\hline 19 & $117.0 \rightarrow 134.0$ & Smectic A & 14 \\
\hline $22 \mathrm{c}$ & $123.6 \rightarrow 135.5$ & Smectic A & 15 \\
\hline 22d & $119.6 \rightarrow 135.0$ & Smectic A & \\
\hline $22 e$ & $117.2 \rightarrow 133.8$ & Smectic A & \\
\hline $22 f$ & $75.1 \rightarrow 108.8$ & Smectic A & \\
\hline $22 \mathrm{~g}$ & $100.3 \rightarrow 116.0$ & Smectic A & \\
\hline $22 \mathrm{~h}$ & $100.3 \rightarrow 107.5$ & Smectic A & \\
\hline $22 \mathbf{i}$ & $70.5 \rightarrow 158.3$ & Smectic A & \\
\hline $22 j$ & $94.8 \rightarrow 107.3$ & Smectic A & \\
\hline $25 / 24 a$ & $108.0 \rightarrow 117.0$ & Smectic A & 20 \\
\hline $25 / 24 b$ & $117.0 \rightarrow 135.0$ & Smectic A & \\
\hline 40d & $231.2 \rightarrow 249.5$ & Smectic A & 22 \\
\hline $40 \mathrm{e}$ & $220.5 \rightarrow 241.4$ & Smectic A & \\
\hline $40 f$ & $223.7 \rightarrow 243.3$ & Smectic A & \\
\hline $40 \mathrm{~g}$ & $212.8 \rightarrow 234.9$ & Smectic A & \\
\hline $40 h$ & $192.0 \rightarrow 236.4$ & Smectic A & \\
\hline $57 \mathbf{b}, \mathbf{d} / 47 \mathbf{a}$ & $154.9 \rightarrow 84.8$ & Smectic A & 23 \\
\hline $57 \mathrm{c}, \mathrm{d} / 47 \mathrm{a}$ & $120.3 \rightarrow 74.9$ & Smectic A & \\
\hline $57 \mathrm{~b}, \mathrm{~d} / 47 \mathrm{a}^{*}$ & $123.8 \rightarrow 70.1$ & Smectic A & \\
\hline $57 \mathrm{c}, \mathrm{d} / 47 \mathrm{a}^{*}$ & $82.1 \rightarrow 60.8$ & Smectic A & \\
\hline $57 b, d * / 47 a$ & $126.3 \rightarrow 64.9$ & Smectic A & \\
\hline $57 c, d^{*} / 47 a$ & $73.5 \rightarrow 35.2$ & Smectic A & \\
\hline $57 b, d * / 47 a^{*}$ & $68.5 \rightarrow 41.5$ & Smectic A & \\
\hline $56 a(8)$ & $104.2 \rightarrow 109.3$ & Smectic C & 30 \\
\hline $56 a(10)$ & $101.6 \rightarrow 127.6$ & Smectic C & \\
\hline $56 a(12)$ & $102.7 \rightarrow 137.2$ & Smectic C & \\
\hline $56 a(16)$ & $106.9 \rightarrow 134.4$ & Smectic C & \\
\hline $56 \mathrm{~b}(8)$ & $75.7 \rightarrow 98.3$ & Smectic C & \\
\hline $56 \mathrm{~b}(10)$ & $78.1 \rightarrow 96.9$ & Smectic C & \\
\hline $56 b(12)$ & $89.1 \rightarrow 96.1$ & Smectic C & \\
\hline $82 b$ & $80.0 \rightarrow 52.0$ & Smectic A & 35 \\
\hline $82 \mathrm{c}$ & $82.0 \rightarrow 74.0$ & Smectic A & \\
\hline $82 d$ & $81.0 \rightarrow 65.0$ & Smectic A & \\
\hline $82 \mathrm{e}$ & $92.0 \rightarrow 78.0$ & Smectic A & \\
\hline $84 b / 85 a$ & $60.8 \rightarrow 37.8$ & Smectic A & 37 \\
\hline $84 b / 85 b$ & $117.7 \rightarrow 93.6$ & Smectic A & \\
\hline $84 b / 85 c$ & $134.0 \rightarrow 78.6$ & Smectic A & \\
\hline $84 b / 85 d$ & $84.4 \rightarrow 47.2$ & Smectic A & \\
\hline $84 b / 85 e$ & $91.6 \rightarrow 55.2$ & Smectic A & \\
\hline $84 b / 85 f$ & $106.7 \rightarrow 64.2$ & Smectic A & \\
\hline $84 f / 85 a$ & $68.7 \rightarrow 11.6$ & Smectic A & \\
\hline $89 a / 90 a$ & $108.8 \rightarrow 134.4$ & Smectic C & 40 \\
\hline $89 a / 90 b$ & $121.3 \rightarrow 135.0$ & Smectic C & \\
\hline $89 a / 90 c$ & $123.8 \rightarrow 140.1$ & Smectic C & \\
\hline 89a/90d & $121.9 \rightarrow 137.5$ & Smectic C & \\
\hline $89 b / 90 a$ & $131.3 \rightarrow 143.8$ & Smectic C & \\
\hline $89 b / 90 b$ & $129.4 \rightarrow 143.2$ & Smectic C & \\
\hline $89 b / 90 c$ & $128.2 \rightarrow 136.7$ & Smectic C & \\
\hline 89b/90d & $123.8 \rightarrow 135.7$ & Smectic C & \\
\hline $89 c / 90 a$ & $118.8 \rightarrow 138.8$ & Smectic C & \\
\hline $89 c / 90 b$ & $128.2 \rightarrow 140.1$ & Smectic C & \\
\hline $89 c / 90 c$ & $131.9 \rightarrow 140.0$ & Smectic C & \\
\hline $89 c / 90 d$ & $128.8 \rightarrow 140.8$ & Smectic C & \\
\hline $89 c / 90 a$ & $129.4 \rightarrow 139.5$ & Smectic C & \\
\hline
\end{tabular}


Table 2 (Contd.)

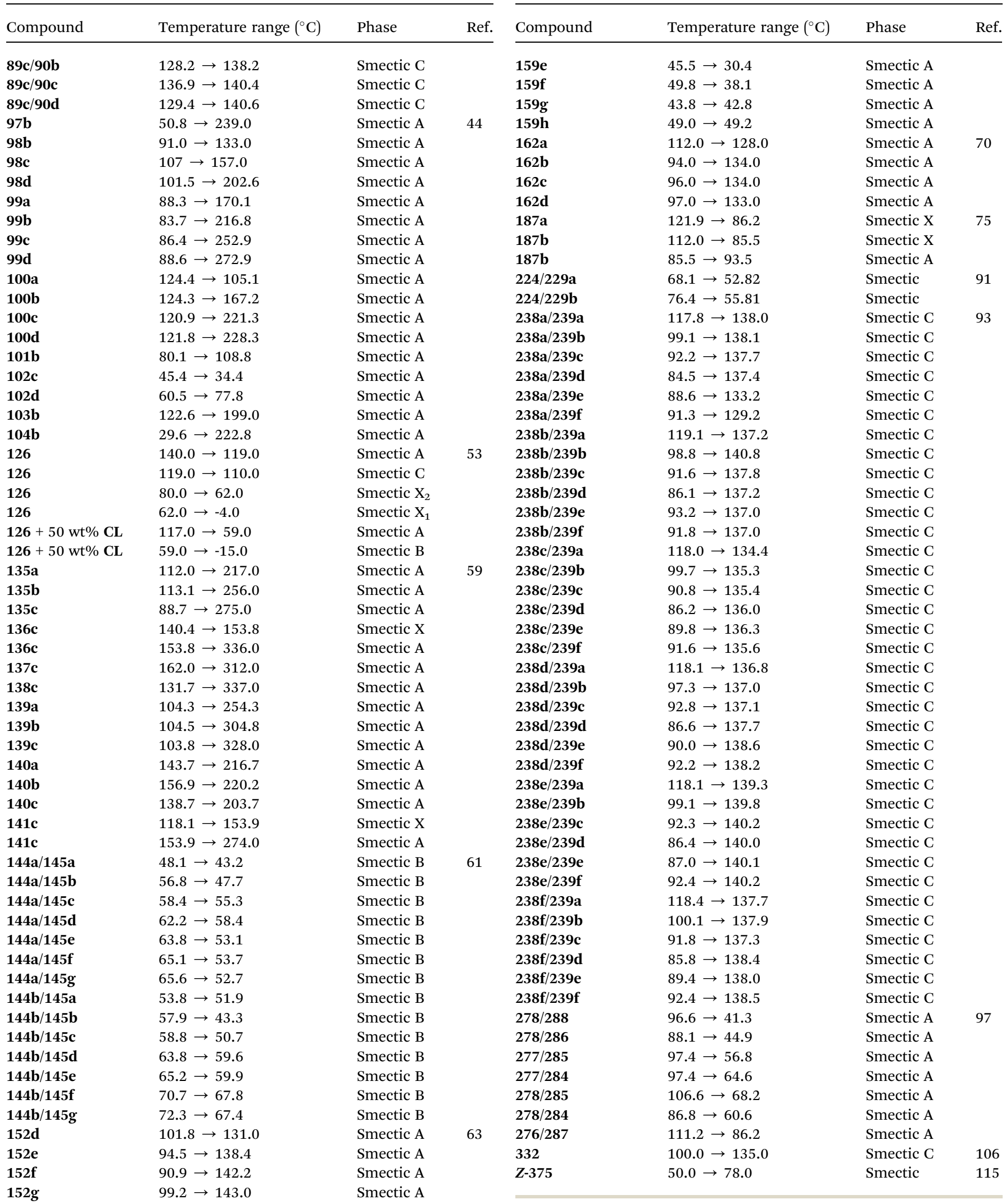

152h $\quad 102.1 \rightarrow 142.2$

152i $\quad 104.4 \rightarrow 137.3$

$159 \mathrm{c}$

159d
$46.5 \rightarrow 44.8$

$43.5 \rightarrow 38.0$
Table 2 (Contd.) 
hexagonal phases, respectively. After the first heating cycle, derivative 311c does not form a crystal phase, i.e. it continues to exist in columnar phase after cooling to room temperature. Among dithiadiazole derivatives 312a-d, derivative 312a is nonmesogenic in nature, and all the other derivatives exhibit mesophases. Derivatives 312b and 312d exhibited columnar rectangular phases, whereas compound 312c exhibited columnar hexagonal and columnar rectangular phases. In this series, derivatives 312c and 312d exhibited columnar rectangular phases at room temperature after the first heating cycle. They also compared these derivatives with the benzene-based

Table 3 List of compounds which exhibit columnar phases and their temperature ranges

\begin{tabular}{|c|c|c|c|}
\hline Compound & Temperature range $\left({ }^{\circ} \mathrm{C}\right)$ & Phase & Ref. \\
\hline $328 a$ & $124.0 \rightarrow 187.0$ & Achiral $I a \overline{3} d$ phase & 41 \\
\hline $328 \mathrm{a}$ & $187.0 \rightarrow 196.0$ & Chiral I432 phase & \\
\hline $328 b$ & $128.0 \rightarrow 201.0$ & Chiral I432 phase & \\
\hline $328 \mathrm{c}$ & $123.0 \rightarrow 191.0$ & Chiral I432 phase & \\
\hline 328d & $92.0 \rightarrow 184.0$ & Chiral $I 432$ phase & \\
\hline $196 f$ & $122.2 \rightarrow 138.8$ & Columnar rectangular & 80 \\
\hline $196 \mathrm{~g}$ & $\mathrm{rt} \rightarrow 107.2$ & Columnar rectangular & \\
\hline $196 h$ & $\mathrm{rt} \rightarrow 113.6$ & Columnar rectangular & \\
\hline $196 \mathbf{i}$ & $\mathrm{rt} \rightarrow 90.84$ & Columnar rectangular & \\
\hline 196j & $100.9 \rightarrow 114.9$ & Columnar rectangular & \\
\hline 196k & $129.2 \rightarrow 137.8$ & Columnar rectangular & \\
\hline 1961 & $104.7 \rightarrow 117.9$ & Columnar rectangular & \\
\hline 196m & $\mathrm{rt} \rightarrow 93.7$ & Columnar rectangular & \\
\hline $204 a$ & $\mathrm{rt} \rightarrow 110.0$ & Columnar hexagonal & 81 \\
\hline $204 b$ & $\mathrm{rt} \rightarrow 87.2$ & Columnar hexagonal & \\
\hline 204c & $\mathrm{rt} \rightarrow 71.6$ & Columnar hexagonal & \\
\hline 204d & $\mathrm{rt} \rightarrow 74.3$ & Columnar hexagonal & \\
\hline 204e & $\mathrm{rt} \rightarrow 81.3$ & Columnar hexagonal & \\
\hline $208 b$ & $105.5 \rightarrow 125.2$ & Columnar orthorhombic & 82 \\
\hline $208 c$ & $105.1 \rightarrow 121.4$ & Columnar orthorhombic & \\
\hline 208d & $102.6 \rightarrow 120.5$ & Columnar orthorhombic & \\
\hline $208 \mathrm{e}$ & $101.6 \rightarrow 118.2$ & Columnar orthorhombic & \\
\hline $208 f$ & $99.3 \rightarrow 113.3$ & Columnar orthorhombic & \\
\hline 211a & $123.0 \rightarrow 57.0$ & Columnar rectangular & 83 \\
\hline 211b & $108.0 \rightarrow 91.0$ & Columnar hexagonal & \\
\hline 211c & $\mathrm{rt} \rightarrow 66.0$ & Columnar hexagonal & \\
\hline $215 c$ & $35.7 \rightarrow 2.4$ & Columnar hexagonal & 85 \\
\hline 216c & $35.5 \rightarrow-18.0$ & Columnar hexagonal & \\
\hline $236 a$ & $89.48 \rightarrow 153.02$ & Oblique columnar & 92 \\
\hline $236 b$ & $88.74 \rightarrow 135.77$ & Ordered columnar hexagonal & \\
\hline $236 b$ & $135.77 \rightarrow 149.14$ & $\begin{array}{l}\text { Disordered columnar } \\
\text { hexagonal }\end{array}$ & \\
\hline $236 \mathrm{c}$ & $74.95 \rightarrow 120.36$ & Ordered columnar hexagonal & \\
\hline $236 \mathrm{c}$ & $120.36 \rightarrow 140.31$ & $\begin{array}{l}\text { Disordered columnar } \\
\text { hexagonal }\end{array}$ & \\
\hline 236d & $112.8 \rightarrow 50.3$ & Ordered columnar hexagonal & \\
\hline 311c & $75.8 \rightarrow 45.3$ & Columnar rectangular 1 & 102 \\
\hline 311c & $45.3 \rightarrow \mathrm{rt}$ & Columnar rectangular 2 & \\
\hline 311d & $92.2 \rightarrow 42.1$ & Columnar hexagonal & \\
\hline $312 b$ & $126.7 \rightarrow 99.1$ & Columnar rectangular & \\
\hline $312 \mathrm{c}$ & $95.6 \rightarrow 70.0$ & Columnar hexagonal & \\
\hline $312 c$ & $70.0 \rightarrow 43.6$ & Columnar rectangular 1 & \\
\hline $312 c$ & $43.6 \rightarrow \mathrm{rt}$ & Columnar rectangular 2 & \\
\hline 312d & $119.7 \rightarrow 73.0$ & Columnar rectangular & \\
\hline 312d & $73.0 \rightarrow 57.3$ & Columnar rectangular 1 & \\
\hline 312d & $57.3 \rightarrow \mathrm{rt}$ & Columnar rectangular & \\
\hline
\end{tabular}

bent-shaped molecules which were reported by Tang et al. ${ }^{\mathbf{4 6 b}}$ and Pathak et al. ${ }^{110}$ The structures of benzene-based molecules 313 and 314a-b are shown in Fig. 18. Benzene-based hexacatenar compound $\mathbf{3 1 3}$ exhibited a narrow range of hexagonal columnar phase and was crystalline at room temperature, while the corresponding hexacatenar 311a displayed a wide range of rectangular columnar phase, including room temperature. Similarly, compound 314a exhibited monomesomorphic oblique columnar phase, in contrast with the compound 312c, which displayed high-stability rectangular columnar and hexagonal columnar mesophases. Oblique columnar phase was exhibited by the benzene-based hexacatenar compound 314b, while rectangular columnar phase was observed in hexacatenar 312d. Finally, they concluded that the stability of the mesophase was enhanced by introducing pyridine as a central core, which also decreased the melting temperature. High stability and a wider range of mesophase were observed in thiadiazolebased hexacatenars in both the pyridine and benzene-based bent molecules.

Pfletscher et al. ${ }^{\mathbf{1 1 1}}$ prepared forty-nine hydrogen bonded complexes by mixing hydrogen donor phenol derivatives such as phenol (315), resorcinol (317), catechol (318), hydroquinone (316), hydroxyhydroquinone (320), pyrogallol (319), and phloroglucinol (321) with the corresponding molar ratios of hydrogen

Table 4 List of compounds which exhibit TGBA* (twisted-grainboundary) phase and their temperature ranges

\begin{tabular}{llll}
\hline Compound & Temperature range $\left({ }^{\circ} \mathrm{C}\right)$ & Phase & Ref. \\
\hline $\mathbf{1 5 a}$ & $112.2 \rightarrow 113$ & TGBA $^{*}$ & 13 \\
$\mathbf{1 5 b}$ & $115.3 \rightarrow 116.5$ & TGBA $^{*}$ & \\
$\mathbf{8 4 f} / \mathbf{8 5 b}$ & $91.7 \rightarrow 61.8$ & TGBA $^{*}$ & 37 \\
$\mathbf{8 4 f} / \mathbf{8 5 c}$ & $75.8 \rightarrow 67.9$ & TGBA $^{*}$ & \\
$\mathbf{8 4 f} / \mathbf{8 5 d}$ & $69.7 \rightarrow 17.7$ & TGBA $^{*}$ & \\
$\mathbf{8 4 f} / \mathbf{8 5 f}$ & $81.8 \rightarrow 22.9$ & TGBA $^{*}$ &
\end{tabular}

Table 5 List of compounds which exhibit blue phase (BP*) and their temperature ranges

\begin{tabular}{|c|c|c|c|}
\hline Compound & Temperature range $\left({ }^{\circ} \mathrm{C}\right)$ & Phase & Ref. \\
\hline 15b & $136.5 \rightarrow 137.5$ & $\mathrm{BP}^{*}$ & 13 \\
\hline $57 b, d * / 47 a$ & $150.3 \rightarrow 147.9$ & $\mathrm{BP}^{*}$ & 23 \\
\hline $57 c, d * / 47 a$ & $96.9 \rightarrow 91.3$ & $\mathrm{BP}^{*}$ & \\
\hline $57 b, d^{*} / 47 a^{*}$ & $89.6 \rightarrow 84.9$ & $\mathrm{BP}^{*}$ & \\
\hline $57 \mathrm{c}, \mathrm{d}^{*} / 47 \mathrm{a}^{*}$ & $70.3 \rightarrow 64.3$ & $\mathrm{BP}^{*}$ & \\
\hline 278/285 & $151.2 \rightarrow 137.5$ & $\mathrm{BP}^{*}$ & 97 \\
\hline
\end{tabular}

Table 6 List of compounds which exhibit B1 phase and their temperature ranges

\begin{tabular}{llll}
\hline Compound & Temperature range $\left({ }^{\circ} \mathrm{C}\right)$ & Phase & Ref. \\
\hline 306b & $102.0 \rightarrow 162.3$ & B1 & 98 \\
306c & $168.5 \rightarrow 179.3$ & B1 &
\end{tabular}


Table 7 List of other compounds which exhibit different phases and their approximate temperature ranges

\begin{tabular}{|c|c|c|c|}
\hline Compound & Temperature range $\left({ }^{\circ} \mathrm{C}\right)$ & Phases & Ref. \\
\hline $31 \mathrm{a}(n)$ & $90.0<$ phases $<190.0$ & Nematic and smectic A & 21 \\
\hline $31 \mathrm{~b}(n)$ & $95.0<$ phases $<170.0$ & Nematic & \\
\hline $30 \mathrm{a}(n)$ & $45.0<$ phases $<100.0$ & Nematic & \\
\hline $30 \mathrm{~b}(n)$ & $35.0<$ phases $<90.0$ & Nematic & \\
\hline $58 \mathrm{~b}-\mathrm{eX} \mathbf{X}_{2}$ & $0.0<$ phases $<185.0$ & $\begin{array}{l}\text { Smectic A, ordered columnar, disordered columnar, lamellar } \\
\text { columnar }\end{array}$ & 25 \\
\hline $59 b / 60 a-e$ & $90.0<$ phases $<125.0$ & Nematic and smectic A & \\
\hline $59 a / 60 a-e$ & $85.0<$ phases $<125.0$ & Nematic and smectic A & \\
\hline 63a-c & $120.0<$ phases $<185.0$ & Smectic C, lamellar and ordered columnar & 28 \\
\hline 64a-c & $180.0<$ phases $<280.0$ & Nematic, disordered and lamellar columnar & \\
\hline $67-68$ & $20.0<$ phases $<70.0$ & Nematic* & 30 \\
\hline $69-72$ & $65.0<$ phases $<240.0$ & Nematic* and smectic A & \\
\hline $128 a / 129 d-e$ & $65.0<$ phases $<130.0$ & Smectic A & \\
\hline $128 b / 129 a-b$ & $60.0<$ phases $<110.0$ & Nematic & \\
\hline $128 b / 129 c-e$ & $60.0<$ phases $<120.0$ & smectic A & \\
\hline $222 / 223 a-h$ & $60.0<$ phase $<100.0$ & Nematic & 90 \\
\hline 268/265 $(0-100 \mathrm{~mol} \%$ of $\mathbf{1 7 8})$ & $40.0<$ phases $<150.0$ & Nematic* and $\mathrm{BP}^{*}$ & 96 \\
\hline $269 / 265(0-100 \mathrm{~mol} \%$ of 178$)$ & $60.0<$ phases $<150.0$ & Nematic* and $\mathrm{BP}^{*}$ & \\
\hline $255 / 265(0-100 \mathrm{~mol} \%$ of $\mathbf{1 7 8})$ & $60.0<$ phases $<150.0$ & Nematic* and $\mathrm{BP}^{*}$ & \\
\hline $272 / 265(0-100 \mathrm{~mol} \%$ of 178$)$ & $80.0<$ phases $<150.0$ & Nematic* and $\mathrm{BP}^{*}$ & \\
\hline $315 / 322 \mathrm{e}$ & $45.0<$ phases $<55.0$ & Nematic and smectic $\mathrm{C}$ & 105 \\
\hline 317/322d-g & $65.0<$ phases $<95.0$ & Smectic & \\
\hline $318 / 322 \mathrm{e}-\mathrm{g}$ & $25.0<$ phases $<55.0$ & Nematic & \\
\hline $321 / 322 d-g$ & $60.0<$ phases $<95.0$ & Nematic & \\
\hline
\end{tabular}

acceptor alkoxyazopyridines (322a-g) and dissolving them in acetone solvent, followed by evaporation of the solvent. The structures of the core units (315-321) and the proton acceptors (322a-g) are presented in Fig. 19. None of the starting materials exhibit liquid crystalline properties. Complexes 316/322a-g, 319/322a-g and 320/322a-g do not exhibit mesogenic properties. Because of successive disaggregation of the hydrogen bonded 320/322a-g complexes, they do not exhibit liquid crystalline properties. In complexes $319 / 322 a-g$, mesophase was not observed due to incomplete formation of the $1: 3$ complexes. The crystal structures of complexes $316 / 322 a-g$ revealed a decreased capacity to slip along each other due to the strongly interdentate packed assemblies, which also decreases the required flexibility for the origin of the liquid crystalline state. The linear arrangements of 316/322a-g and 317/322a-g favor the formation of crystalline and smectic phases, respectively. Dissimilarly bent-shaped structures of 318/322a-g and 319/ $322 \mathrm{a}-\mathbf{g}$ resulted in nematic mesophases. When $n=7$, all the complexes are non-mesogens except for complex 321/322c. In complexes 315/322a-g, only the complex with $n=9(315 / 322 e)$ exhibited smectic phase; all the other complexes are nonmesogens. As the chain length increases in the 318/322a-g complexes, the mesophase stability decreases from $\Delta T=$ $13.6{ }^{\circ} \mathrm{C}$ to $\Delta T=6.8{ }^{\circ} \mathrm{C}$. However, in the $317 / 322 \mathrm{a}-\mathrm{g}$ complexes, the mesophase stability was found to increase from $\Delta T=6.3^{\circ} \mathrm{C}$ to $\Delta T=21.7^{\circ} \mathrm{C}$ as the chain length increased. They also studied the photo-responsive behavior of the hydrogen bonded liquid crystals, which revealed that on irradiation of light at $405 \mathrm{~nm}$, a reversible phase transition occurred from the mesophase into the isotropic phase, where the speed of interchange depends on the morphologies of the phases. Smectic mesophase responded remarkably more slowly than the nematic mesogens. This outcome indicated that separation of the aromatic and aliphatic fragments, as noted in the solid state structure, sustained the generation of stable mesophases.

\subsection{Pyridine-based polycatenar mesogens}

Polycatenar mesogens are a hybrid class of thermotropic liquid crystalline materials which have molecular features of both calamitic and discotic mesogens. Here, the central core has a rod-type molecular structure and two half disc-like structures at both ends. There are different types of polycatenar LCs based on the number of aliphatic chains on both sides of the molecules. The numbers of chains on both sides of the core vary from two to six. In this section, we discuss the available synthetic routes for the preparation of pyridine-based polycatenar mesogens and their properties.

Alaasar et al. ${ }^{\mathbf{4 1}}$ designed and synthesized hydrogen bonded supramolecular complexes with polycatenar structures exhibiting high-powered mirror-symmetry shattering by chirality 
synchronization in chiral "Im $\overline{3} m$ type" cubic phase $\left(\mathrm{Cub}^{[*] / I 432)}\right.$ as well as in a liquid conglomerate $\left(\right.$ Iso $\left._{1}{ }^{[*]}\right)$ at the liquid-liquid transition. Benzoic acid derivatives 325 and azopyridines 327ad were synthesized as shown in Scheme 40. The benzoic acid 325 displays a hexagonal columnar phase between $162{ }^{\circ} \mathrm{C}$ and $246{ }^{\circ} \mathrm{C}$, and 4-(4-alkyloxyphenylazo)pyridines $\mathbf{3 2 7} \mathbf{a}-\mathbf{d}$ are nonmesomorphic in nature. Hexagonal columnar phase was observed in the case of $\mathbf{3 2 5}$, which was ascribed to the formation of dimers by intermolecular hydrogen bonding between the - $\mathrm{COOH}$ groups. An optical negative hexagonal columnar phase was developed due to alignment of the rod-like cores perpendicular to the long axis in the columns. The hydrogen bonded supramolecular complexes 328a-d were prepared by mixing equimolar amounts of $\mathbf{3 2 7} \mathbf{a}-\mathbf{d}$ and $\mathbf{3 2 5}$ followed by melting with stirring. After the crystallization, the product was ground and the process was repeated to afford a homogeneous mixture. All the supramolecular complexes 328a-d exhibited reproducible transition temperatures and homogenous melting. In the supramolecular complexes, a broad cubic liquid crystal phase was induced by suppressing the columnar phases. The complexes 328b-d exhibited cubic phases, whereas in complex 328a, an additional liquid-liquid transition (Iso-Iso ${ }_{1}$ transition) was observed. The liquid phases Iso and $\mathrm{Iso}_{1}$ appeared uniformly dark between the crossed polarizers. However, a gentle rotation of the analyzer by negligible degrees $\left(\mathrm{ca} .-7^{\circ}\right)$ out of the $90^{\circ}$ direction in the $\mathrm{Iso}_{1}$ phase range with respect to the polarizer results in the occurrence of dark and bright zones; by gyration of the analyzer at the same angle in the opposite direction $\left(c a .+7^{\circ}\right)$, the brightness of the zones is the opposite. No changes were observed when rotating the sample between crossed polarizers; this indicated that distinct regions exhibit chiral domains. This provides obvious evidence for chirality synchronization in the Iso $_{1}$ phase $\left(\right.$ Iso $\left._{1}{ }^{[*]}\right)$. In the Iso phases of achiral complexes 328b-d and the Iso phase of 328a at higher temperature, this type of domain was not observed. On melting the crystalline phase at $T=124{ }^{\circ} \mathrm{C}$, achiral $I a \overline{3} d$ phase was formed, which transformed into chiral $I 432$ phase on heating at $T=180^{\circ} \mathrm{C}$ to $187^{\circ} \mathrm{C}$. Optical studies revealed that increasing the alkyl chain length decreases the helical pitch, which becomes irreconcilable with the structure of $I a \overline{3} d$ and leads to the emergence of $I 432$ cubic phase. In the cases of molecules with shorter chain lengths, liquid miscellany was formed, and the desegregation in the midst of the chain and core units was adequately fragile to hinder the development of the long range cubic lattice.

Recently, Pană et al. ${ }^{112}$ prepared flexibly linked bis(pyridinium) salts bearing different counter ions such as $\mathrm{Br}^{-}, \mathrm{PF}_{6}{ }^{-}$, $\mathrm{BF}_{4}{ }^{-}$and $\mathrm{OTf}^{-}$using $N$-alkylated 4-pyridones and 3,4,5-tri$\mathrm{s}($ alkyloxy)benzyl moieties. All the prepared bis(pyridinium) salts exhibited an enantiotropic hexagonal columnar mesophase except the salt of triflate ion, which contains a shorter terminal carbon chain.

\subsection{Pyridine-based polymeric mesogens}

Polymeric mesogens are compounds that blend the properties of polymers with those of mesogens. These mesogens exhibit the anisotropic properties of liquid crystals but retain the unique properties of polymer compounds, such as ultra-thin film forming ability and high strength. Compared to conventional polymers, these materials exhibit excellent mechanical properties and chemical stabilities. In this section, we discuss the available synthetic routes for the preparation of different pyridine-based polymeric mesogens and their properties.

Chen et al. ${ }^{113}$ synthesized a polymer formed by amide linkages containing pyridine and a shape memory polyurethane (331) polymer by treating 1,6-hexanediisocyante (330) with $N, N$ bis(2-hydroxylethyl)isonicotinamine $(329)$ at $80{ }^{\circ} \mathrm{C}$ in dimethyl formamide (DMF) solvent using $0.02 \mathrm{wt} \%$ dibutyltin dilaurate as a catalyst (Scheme 41). Further, a series of hydrogen bonded complexes (332) were prepared by mixing 331 and 4-hexadecyloxybenzoic acid (HOBA) at different mole ratios of HOBA/ 331 in DMF solvent for a specified time, followed by solvent drying under vacuum. The formed supramolecular structure of 332 is shown in Fig. 20. Furthermore, the formation of hydrogen bonds between $\mathbf{3 3 1}$ and HOBA was confirmed by comparing the FT-IR spectra of the parent compounds as well as the prepared hydrogen bonded complexes. These complexes exhibited both shape memory and liquid crystalline properties. The hydrogen bonded complexes maintained the intrinsic smectic $\mathrm{C}$ phase of HOBA at all the mole ratios; a high mole ratio resulted in clearer smectic $\mathrm{C}$ phase and crystalline phases because the sample not only contained hydrogen-bonded HOBA complexes but also HOBA dimers. The morphological examinations showed that complex $\mathbf{3 3 2}$ forms two-phase separated structures due to the presence of an amorphous polyurethane matrix and a HOBA crystalline phase; this may originate from the hydrogen-bonded interactions and free HOBA. Fig. 21 shows the other hydrogen bonds which may occur in supramolecular complex 332.

Chen et $a l .{ }^{114}$ designed and synthesized a supramolecular liquid crystalline shape-memory polyurethane complex (337) by fusing 4-octyloxybenzoic acid (336) with a polymer (335) by hydrogen bonding interactions; they also prepared complexes with different mole ratios $(0.10,0.23,0.38,0.58$, and $0.86 \mathrm{wt} \%)$ of 336/335. These complexes exhibited both multi-shape memory and liquid crystalline properties. The synthesis of compound 335 is given in Scheme 42 . The synthesis of polymer 335 was accomplished using 1,6-hexanediisocyante (330) with $\mathrm{N}, \mathrm{N}$-bis(2-hydroxylethyl)isonicotinamine $(329)$ at $80{ }^{\circ} \mathrm{C}$ in DMF solvent using $0.02 \mathrm{wt} \%$ dibutyltin dilaurate as a catalyst, followed by extension of the chains by adding diphenylmethane diisocyanate (333) and 1,4-butanediol (334) to the reaction mixture; to control the viscosity of the reaction, DMF was added occasionally for another $2 \mathrm{~h}$, and the reaction was maintained for $4 \mathrm{~h}$ to obtain a solution of 10-wt\% 335/DMF. Further, this compound was used to form hydrogen bonds with 336 at different mole ratios of $336 / 335(0.10,0.23,0.38,0.58$, and $0.86 \mathrm{wt} \%)$. These mixtures were stirred for $2 \mathrm{~h}$ to form homogenous solution-phase mixtures; finally, each mixture was added to a Teflon pan and incubated for $24 \mathrm{~h}$ at $80{ }^{\circ} \mathrm{C}$, then dried under a vacuum of 0.1 to $0.2 \mathrm{kPa}$ at $80^{\circ} \mathrm{C}$ for $24 \mathrm{~h}$. Fig. 22 presents the hydrogen bonded complex 337 . The crystallization peak appropriately increases as the content of $\mathbf{3 3 6}$ increases. In the higher $\mathbf{3 3 6}$ content complexes, there is a phase transition 
from nematic to smectic phase; this was identified at $102{ }^{\circ} \mathrm{C}$ to $115^{\circ} \mathrm{C}$, in addition to a $2^{\text {nd }}$ crystallization peak at $94^{\circ} \mathrm{C}$. Thus, it is confirmed that in the prepared complexes, both the liquid crystalline and crystalline properties of $\mathbf{3 3 6}$ were maintained. Shape memory investigation indicated that these complexes have good multi-shape memory effects, incorporating tripleshape memory and quadruple-shape memory behaviors. Because of the combination of multi-shape memory effects and liquid crystalline properties in these complexes, they are potential candidates for applications in smart sensors, smart electronics and smart optical devices.

Al-Lami15 prepared two block copolymers which show supramolecular liquid crystalline properties. 4-Heptyloxy benzoic acid (339) was synthesized using 1-bromoheptane and 4hydroxy benzoic acid (338). 4-Heptyloxy- $N$-pyridine-4-ylbenzamide (340) was synthesized by refluxing 339 with excess thionyl chloride in dry $\mathrm{N}_{2}$ atmosphere for $5 \mathrm{~h}$. Under reduced pressure, the excess thionyl chloride was removed. The obtained acid chloride and 4-aminopyridine were later dissolved in dry pyridine and stirred at room temperature for $24 \mathrm{~h}$, followed by pouring the reaction mixture into dilute hydrochloric acid to obtain the crude product. The obtained crude product was filtered and recrystallized using ethanol. On the other hand, $\alpha, \beta$-bis(4-formyl phenyl-4-oxy)alkanes (342a-f) were prepared by refluxing the required dibromo alkane, 4-hydroxy benzaldehyde (341) and $\mathrm{KOH}$ for $24 \mathrm{~h}$. The crude product was filtered and recrystallized using ethanol. Further, by referring to the report by Ignatious et al. ${ }^{\mathbf{1 1 6}}$ the authors prepared a block copolymer series (344a-f) by refluxing the monomer 2,3,5-diamino benzoic acid (343) and a catalytic amount of glacial acetic acid in ethanol solvent for $2 \mathrm{~h}$. To the same reaction mixture, 1,2-diaminoethane was added slowly, and the mixture was refluxed for $2 \mathrm{~h}$ to afford the block polymers 345a-f. The obtained products were filtered and re-crystallized using ethanol solvent. Furthermore, the hydrogen bonded complexes were prepared by mixing carboxylic acid containing the block copolymer (345a-f) and pyridine-containing monomer (340) in an equimolar ratio; this mixture was dissolved in pyridine, followed by removal of the solvent under vacuum for several days at $80{ }^{\circ} \mathrm{C}$ (Scheme 43). These prepared copolymers have two different flexible spacer lengths, in which the first spacer is fixed at two methylene groups while the other spacer varies from two to seven groups. Using DSC and POM, the mesophase properties of the copolymer were studied. These studies revealed that all the copolymers exhibit nematic phase. It was also found that even-numbered chain lengths have higher transition temperatures than odd-numbered chain lengths. Further, the clearing temperature and melting point were found to gradually decrease as the flexible spacer length increased, in which even members showed slightly higher values. The decreases in the melting point and isotropic temperature are due to the increase in the flexible spacer chain length. Also, the number of possible conformations increased, which resulted in distortion of the cylindrical shapes of the mesogens.

Wang et al. ${ }^{117}$ prepared a series of supramolecular polymer complexes, 347a-d(x). These complexes were prepared by dissolving polymer poly(4-vinylpyridine) (P4VP) and dendron-like small molecules containing phenolic end groups $(\boldsymbol{n C T B}, n=$ $6,10,12,14)$ separately in chloroform; then, these two solutions were mixed to form $5 \mathrm{wt} \%$ solutions and stirred for $12 \mathrm{~h}$ at room temperature. The resulting complexes were maintained at $30{ }^{\circ} \mathrm{C}$ for $24 \mathrm{~h}$ in a vacuum oven. Fig. 23 presents the hydrogen bonded complexes 347a-d(x). These complexes exhibited supramolecular lamellar and hexagonal columnar structures, the stability of which depends on the alkyl chain length, the blending ratio of $\boldsymbol{n C T B}$ per vinylpyridine unit $(\mathbf{x})$ and the thermal treatment. $\mathbf{3 4 7 a}(\mathbf{x})$ exhibits only poorly ordered lamellar phase. However, in the other complexes 347b-d $(n=$ $10,12,14)$, the lamellar structure transformed into a hexagonal columnar structure as $\mathrm{x}$ increased. When the volume fraction of the alkyl tails was about 0.56 to 0.58 , a transition occurred from lamellar to hexagonal columnar phase (a non-reversible orderto-order transition) during the thermal annealing. This is due to the conformational changes in P4VP and the thermal dynamic nature of the hydrogen bonds.

Yang et $a l .{ }^{118}$ synthesized main-chain/side-chain combined liquid crystalline polymers containing mesogen-jacketed liquid crystalline polymers (MJLCPs) as the main chains. In these polymers, a pyridine derivative with a triphenylene (Tp) unit was used as the hydrogen bond acceptor. 2-Vinylbenzene-1,4-dioic acid (348) was synthesized by referring to a previous report by Zhang et al., ${ }^{119}$ and it was stirred at room temperature with 2methylpropan-2-ol (349), N,N-dimethylpyridin-4-amine, and $N, N^{\prime}$-dicyclohexylcarbodiimide in dichloromethane solvent for $24 \mathrm{~h}$. The insoluble matter was removed by filtration and the solvent was evaporated under reduced pressure to afford di-tertbutyl 2-vinylterephthalate (350). 2,3-Dimethyl-2,3-butanediol was refluxed with 4-(methoxycarbonyl)phenylboronic acid in THF solvent for $2 \mathrm{~h}$ at $70{ }^{\circ} \mathrm{C}$ and the solvent was evaporated under reduced pressure to afford methyl 4-(4,4,5,5-tetramethyl1,3,2-dioxaborolan-2-yl)benzoate. 2,5-Dibromostyrene (354) was synthesized by referring to a previous report by Qu et al. $;^{\mathbf{1 2 0}}$ then, it was taken in a three-necked flask to which 4-methyl-(4,4,5,5tetramethyl-1,3,2-dioxaborolan-2-yl)benzoate, hydroquinone, potassium carbonate and $\mathrm{Pd}\left(\mathrm{PPh}_{3}\right)_{4}$ were added. The reaction was performed under a continuous stream of argon, and water and toluene were injected during the course of the reaction. Later, this reaction mixture was stirred for $48 \mathrm{~h}$ at $110{ }^{\circ} \mathrm{C}$. The separated organic layer was dried using anhydrous magnesium sulphate $\left(\mathrm{MgSO}_{4}\right)$ and the solvent was evaporated under reduced pressure to afford 2,5-bis(4-methoxycarbonyl phenyl) styrene (357). Further, this compound was dissolved in ether and potassium tert-butoxide was added slowly. The mixture was stirred for $2 \mathrm{~h}$ at ambient temperature, washed with water and dried using $\mathrm{MgSO}_{4}$. The solvent was evaporated after filtration to afford 2,5-bis(4-tert-btuoxylcarbonyl phenyl)styrene (358). $N, N, N^{\prime}, N^{\prime \prime}, N^{\prime \prime}$-Pentamethyldiethylenetriamine (PMDETA), monomer 350, (1-bromoethyl)benzene, dry chlorobenzene, and CuBr were taken in a polymerization tube. The tube was sealed under vacuum after three freeze-pump-thaw cycles and later maintained in a thermostatted oil bath for $24 \mathrm{~h}$ at $110{ }^{\circ} \mathrm{C}$. The tube was quenched in liquid nitrogen to stop the polymerization and was then maintained in ambient conditions. The solution was diluted using THF solvent, and copper salt was 
removed by passing it through the alumina column. Finally, 359 was precipitated from methanol and dried. Then, it was dissolved in chloroform, and trifluoroacetic acid was added slowly at ambient temperature. The solution was maintained for $24 \mathrm{~h}$ with stirring and the solvent was evaporated under reduced pressure to afford polymer 360 (Scheme 44). By referring to the report by Xing et al., ${ }^{121}$ 2-hydroxyl-3,6,7,10,11-pentakis(hexyloxy) triphenylene (364) was synthesized, and it was refluxed with 1,6dibromohexane, potassium iodide, and potassium carbonate in acetonitrile solvent. After the reaction, the mixture was filtered and the filtrate was collected; the solvent was evaporated under reduced pressure to afford compound 366 , followed by refluxing compound 366 with 4-hydroxy pyridine (367) in the presence of potassium iodide and potassium carbonate in DMF solvent to yield compound 368 (Scheme 45). Finally, the expected complexes were prepared by mixing different molar ratios of 368 to the $-\mathrm{COOH}$ groups in the repeating units in pyridine solvent and stirring for $24 \mathrm{~h}$ at $60{ }^{\circ} \mathrm{C}$; then, the solvent was allowed to evaporate slowly for 3 days at $50{ }^{\circ} \mathrm{C}$, and the product was dried under vacuum at $60{ }^{\circ} \mathrm{C}$ for another $24 \mathrm{~h}$. The phase behavior of the synthesized complexes strongly depends on the rigidity of the side-chain core of the MJLCPs and the amount of hydrogen bond acceptor. In the $\mathbf{3 5 2} / \mathbf{3 6 8 x}$ complex, the simple columnar phase transforms to a hierarchical nanostructure containing a hexagonal columnar phase arising from the entire polymer chain and a discotic nematic phase assigned to the $\mathbf{T p}$ moieties. Meanwhile, in the $\mathbf{3 6 0 / 3 6 8 x}$ complex, the simple smectic A phase transforms into a hierarchical nanostructure containing a smectic A phase arising from the entire polymer chain and a discotic nematic phase assigned to the Tp moieties when $\mathbf{x}$ is increased to 1 . Hence, they suggested that when the 368 content is comparatively low, weak interactions between the Tp moieties cause the complexes to behave like independent MJLCPs, and that the Tp moieties can be strongly considered as side chain constituents of the MJLCPs. The interactions between the $\mathbf{T p}$ moieties become stronger as the 368 content increases, and both types of mesogens can act separately to exhibit their solitary self-organizing structures.

Zhu et al. ${ }^{122}$ prepared $\alpha$-cyanostilbene containing hydrogen bonded supramolecular polymers. 3,4,5-trihydroxybenzaldehyde (369) monohydrate was dissolved in dry DMF; to this solution, $\mathrm{K}_{2} \mathrm{CO}_{3}$ and $\mathrm{KI}$ were added, and the mixture was stirred at $80{ }^{\circ} \mathrm{C}$. To this reaction mixture, 1-bromododecane (370) was added dropwise; then, the reaction was continued overnight. After completion of the reaction, the reaction mixture was added to brine solution and extracted using dichloromethane. Using magnesium sulphate, the organic layer was dried, and the solvent was evaporated using a rotary evaporator. Finally, the pure 3,4,5-tris(dodecyloxy)benzaldehyde (371) was obtained by column chromatography. 4-Hydroxybenzeneboronic acid (373) and 4-bromophenylacetonitrile (372) were dissolved in THF; later, sodium carbonate solution was added. Under $\mathrm{N}_{2}, \operatorname{Pd}\left(\mathrm{PPh}_{3}\right)_{4}$ was added, and the reaction mixture was refluxed for $12 \mathrm{~h}$. After completion of the reaction, the reaction mixture was cooled to room temperature; then, the mixture was neutralized with $\mathrm{HCl}$. The product was extracted using brine/ethyl acetate, and the organic phase was dried using anhydrous magnesium sulphate. Finally, pure $\left(4^{\prime}\right.$ hydroxybiphenyl-4-yl)acetonitrile (374) was obtained by column chromatography. Compound 371, compound 374, and sodium hydroxide were dissolved in anhydrous methanol and stirred for $12 \mathrm{~h}$ at $50{ }^{\circ} \mathrm{C}$ under nitrogen atmosphere. After completion of the reaction, the reaction mixture was cooled to room temperature; then, it was neutralized using hydrochloric acid to obtain the product (Z)-2-(4'-hydroxybiphenyl-4-yl)-3-(3,4,5-tris(dodecyloxy)phenyl)acrylonitrile (Z-375) (Scheme 46). Irradiating a concentrated solution of $Z-375$ in $\mathrm{CHCl}_{3}$ with UV light of $365 \mathrm{~nm}$ for $3 \mathrm{~h}$ yielded a mixture of $Z-375$ and E-375. Using column chromatography, $\boldsymbol{E}$-375 was separated. P4VP and 375 ( $Z$ or $E$-isomers) were dissolved in chloroform separately to obtain solutions of $5 \mathrm{wt} \% .375$ and P4VP solutions were mixed in appropriate quantities according to $\mathbf{x}$ in order to obtain $\operatorname{P4VP}(375) x$ aggregations, which were then stirred at room temperature for $24 \mathrm{~h}$. After completion of the reaction, the solvent was evaporated slowly and the product was maintained in a vacuum oven at $35^{\circ} \mathrm{C}$. Thus, the supramolecular complexes were obtained (Scheme 47). Cylindrical assemblies formed in the $\boldsymbol{Z}$-375-based complexes due to the wrapping of hydrogenbonded dendritic $Z \mathbf{Z}-375$ molecules around the P4VP chains. The hexagonal packing was due to the parallel arrangement of supramolecular cylinders. Columnar packing was not favored by the bent-shaped $E$-isomer. The lamellar arrangement was ascribed to the micro-phase partition between the aliphatic non-polar part and aromatic polar part in the polymer aggregations. At the same time, the hydrogen-bonding interactions were hindered to some extent due to the bulky shape of the $E$ isomer. The corresponding $\operatorname{P4VP}(Z-375) x$ and $\operatorname{P4VP}(E-375) x$ aggregates exhibited divergent stimuli-responsive properties and self-assembly behavior. $\mathbf{P 4 V P}(Z-375) \boldsymbol{x}$ exhibited a hexagonal columnar structure at $0.4 \leq x \leq 1.0$; at the same time, a lamellar structure developed for $\mathbf{P 4 V P}(\boldsymbol{E}-375) \boldsymbol{x}$ at $0.3 \leq x \leq 0.7$. The $\mathbf{P 4 V P}(Z-375) 0.5$ film exhibited irreversible switching and increased fluorescence emission upon UV illumination. Thus, by controlling the configuration of molecules in supramolecular polymer systems, the functional responsive behaviors and phase structures can be readily tuned at the same time.

\section{Optical textures and temperature ranges of the mesophases}

The textures of various mesophases observed by POM for these pyridine-based compounds are shown in Fig. 24-27; also, the compounds which exhibit various mesophases and their temperature ranges are tabulated in Tables 1-7. As a whole, this paper provides a review of the latest advances in the area of pyridine-based liquid crystals with respect to the design of their molecular architectures and the establishment of suitable synthetic protocols, correlation of the shape and the type of mesophase, improvisation of the mesophase range, the roles of substituents, tuning of the melting and clearing temperatures with the aid of varying alkoxy chain lengths, preparation of hydrogen bonded complexes, photophysical properties, and device applications. 


\section{Summary and conclusions}

In this review article, prominence has been provided to the available synthetic routes and thermotropic properties of various pyridine-based molecules. In addition, their photoisomerisation, opto-electric properties, blue phase ranges and structure-property relationships have been explained in some cases. Most of the molecules with short chain lengths exhibit nematic phase, whereas smectic A phase is observed for molecules bearing longer chain lengths. This is because the ratio of lateral to terminal attraction between the molecules increases as the chain length increases. As the chain length increases, the probability of layer arrangement during the melting process (crystal-liquid crystalline transition) increases due to the weakened terminal attractions. Further, it has been noted that the incorporation of a pyridine system into a molecular architecture not only alters its mesogenic behavior but also modifies its electronic and dielectric properties. Therefore, these molecules are currently gaining a wide range of applications in the fields of photovoltaic devices, organic LEDs, and field effect transistors. Moreover, some new generation batteries have started using ionic liquid crystals as electrolytes. Furthermore, molecules possessing azo linkages exhibit phase transitions upon UV light irradiation. Generally, this phase transition is because of photoisomerisation of azo compounds from trans to cis configuration as the bent-shaped isomers destabilize the liquid crystal phases. In these molecules, mesogenic textures emerge immediately after stopping the UV irradiation, and the textures are fully retrieved within a few seconds. Hence, these molecules are more suitable for photonic applications. As a whole, this article will help researchers to establish synthetic strategies to prepare new pyridine-based mesogenic molecules.

\section{Future prospects}

The exigency of advancing technological developments is currently increasing. To meet these demands, the sustained design and preparation of new mesogenic molecules is highly desirable. One great challenge in the future is to synthesize these molecules in a greener way, i.e. using ionic liquids, ultrasonication, etc. Further, the design of new molecular entities encompassing both mesomorphic and luminescence properties in the future will establish these materials as excellent contenders for opto-electronic applications. Furthermore, the design of pyridine-based luminescent mesomorphic molecules may be an innovative strategy to enlarge the viewing angles of twisted nematic liquid crystal displays and also to overcome limitations such as loss of contrast at high temperature and high heat generation due to high consumption of electricity. Finally, the refinement of this pyridine platform to tailor the exigency of various technological devices based on optical and electrical properties can be envisioned.

\section{Conflicts of interest}

There are no conflicts to declare.

\section{Acknowledgements}

This research was supported by the Science \& Engineering Research Board (SERB) under Young Scientists Scheme (Project file no: YSS/2014/000835), Govt. of India, New Delhi.

\section{References}

1 M. P. Aldred, P. Vlachos, D. Dong, S. P. Kitney, W. Chung Tsoi, M. O'Neill and S. M. Kelly, Heterocyclic Reactive Mesogens: Synthesis, Characterisation and Mesomorphic Behaviour, Liq. Cryst., 2005, 32(8), 951-965.

2 A. Seed, Synthesis of Self-Organizing Mesogenic Materials Containing a Sulfur-Based Five-Membered Heterocyclic Core, Chem. Soc. Rev., 2007, 36(12), 2046-2069.

3 V. V. Titov and A. I. Pavlyuchenko, Therotropic Liquid Crystals in the Heterocyclic Series (Review), Chem. Heterocycl. Compd., 1980, 16(1), 1-13.

4 M. Lehmann, G. Kestemont, R. G. Aspe, C. Buess-Herman, M. H. J. Koch, M. G. Debije, J. Piris, M. P. De Haas, J. M. Warman, M. D. Watson, et al., High Charge-Carrier Mobility in $\pi$-Deficient Discotic Mesogens: Design and Structure-Property Relationship, Chem.-Eur. J., 2005, 11(11), 3349-3362.

5 M. Takase, V. Enkelmann, D. Sebastiani, M. Baumgarten and K. Müllen, Annularly Fused Hexapyrrolohexaazacoronenes: An Extended $\pi$ System with Multiple Interior Nitrogen Atoms Displays Stable Oxidation States, Angew. Chem., Int. Ed., 2007, 46(29), 5524-5527.

6 L.-L. Lai, C.-H. Wang, W.-P. Hsieh and H.-C. Lin, Synthesis and Characterization of Liquid Crystalline Molecules Containing the Quinoline Unit, Mol. Cryst. Liq. Cryst., 1996, 287(1), 177-181.

7 T. Ghosh and M. Lehmann, Recent Advances in Heterocycle-Based Metal-Free Calamitics, J. Mater. Chem. C, 2017, 5(47), 12308-12337.

8 T. Geelhaar and E. Merck, Ferroelectric Mixtures and Their Physico-Chemical Properties, Ferroelectrics, 1988, 85(1), 329-349.

9 M. O'Neill and S. M. Kelly, Liquid Crystals for Charge Transport, Luminescence, and Photonics, Adv. Mater., 2003, 15(14), 1135-1146.

10 X. bing Zhang, B. chen Tang, P. Zhang, M. Li and W. jing. Tian, Synthesis and Characterization of 1,3,4Oxadiazole Derivatives Containing Alkoxy Chains with Different Lengths, J. Mol. Struct., 2007, 846(1-3), 55-64.

11 J. A. Nash and G. W. Gray, Studies of Some Heterocyclic Mesogens, Mol. Cryst. Liq. Cryst., 1974, 25(3-4), 299-321.

12 Z. Liu, J. Han, J. Zhang, Z. Yu, T. Li and S. Zhang, Synthesis and Mesomorphic Properties of New Fluorinated Hydrogen-Bonded Supramolecular Liquid Crystals, Monatsh. Chem., 2014, 145(1), 71-77.

13 W. L. He, M. J. Wei, H. Yang, Z. Yang, H. Cao and D. Wang, Flexible H-Bonded Liquid-Crystals with Wide Enantiotropic Blue Phases, Phys. Chem. Chem. Phys., 2014, 16(12), 56225626. 
14 E. Zapp, E. Westphal, H. Gallardo, B. de Souza and I. Cruz Vieira, Liquid Crystal and Gold Nanoparticles Applied to Electrochemical Immunosensor for Cardiac Biomarker, Biosens. Bioelectron., 2014, 59, 127-133.

15 Y. Chen, H. Yu, L. Zhang, H. Yang and Y. Lu, Photoresponsive Liquid Crystals Based on Halogen Bonding of Azopyridines, Chem. Commun., 2014, 50(68), 9647-9649.

16 V. A. Mallia, P. K. Sudhadevi Antharjanam and S. Das, Synthesis and Studies of Some 4-Substituted Phenyl-4'Azopyridine-Containing Hydrogen-Bonded Supramolecular Mesogens, Liq. Cryst., 2003, 30(2), 135-141.

17 D. A. Paterson, A. Martínez-Felipe, S. M. Jansze, A. TM Marcelis, J. MD Storey and C. T. Imrie, New Insights into the Liquid Crystal Behaviour of Hydrogen-Bonded Mixtures Provided by Temperature-Dependent FTIR Spectroscopy, Liq. Cryst., 2015, 42(5-6), 928-939.

18 S. M. Jansze, A. Martínez-Felipe, J. M. D. Storey, A. T. M. Marcelis and C. T. Imrie, A Twist-Bend Nematic Phase Driven by Hydrogen Bonding, Angew. Chem., Int. Ed., 2015, 54(2), 643-646.

19 M. J. Wallage, Supramolecular Dimeric Liquid Crystals. the Liquid Crystalline Behaviour of Mixtures of $\alpha$-(4-Pyridyloxy)$\omega$-[4-(4-Butylphenylazo)phenoxy] Alkanes and 4Octyloxybenzoic Acid, J. Mater. Chem., 1997, 7(7), 11631167.

20 A. Martínez-Felipe and C. T. Imrie, The Role of Hydrogen Bonding in the Phase Behaviour of Supramolecular Liquid Crystal Dimers, J. Mol. Struct., 2015, 1100, 429-437.

21 W. L. He, Q. Huang, Z. Yang, H. Cao, D. Wang and H. Yang, Effect of Bent-Shape and Calamitic-Shape of HydrogenBonded Mesogens on the Liquid Crystalline Properties, Liq. Cryst., 2015, 42(8), 1191-1200.

22 A. K. Al-Lami, Preparation and Mesomorphic Characterization of Supramolecular Hydrogen-Bonded Dimer Liquid Crystals, Polycyclic Aromat. Compd., 2016, 36(3), 197-212.

23 C. L. Wei, T. C. Chen, P. Raghunath, M. C. Lin and H. C. Lin, Hydrogen-Bonded Effects on Supramolecular Blue Phase Liquid Crystal Dimeric Complexes, RSC Adv., 2015, 5(67), 54629-54637.

24 C. L. Wei, T. C. Chen, P. Raghunath, M. C. Lin and H. C. Lin, Novel Asymmetrical Single- and Double-Chiral Liquid Crystal Diads with Wide Blue Phase Ranges, RSC Adv., 2015, 5(6), 4615-4622.

25 S. Cospito, A. Beneduci, L. Veltri, M. Salamonczyk and G. Chidichimo, Mesomorphism and Electrochemistry of Thienoviologen Liquid Crystals, Phys. Chem. Chem. Phys, 2015, 17(27), 17670-17678.

26 F. Fernandez-Palacio, M. Poutanen, M. Saccone, A. Siiskonen, G. Terraneo, G. Resnati, O. Ikkala, P. Metrangolo and A. Priimagi, Efficient Light-Induced Phase Transitions in Halogen-Bonded Liquid Crystals, Chem. Mater., 2016, 28(22), 8314-8321.

27 D. W. Bruce, P. Metrangolo, F. Meyer, C. Präsang, G. Resnati, G. Terraneo and A. C. Whitwood, Mesogenic, Trimeric, Halogen-Bonded Complexes from
Alkoxystilbazoles and 1,4-Diiodotetrafluorobenzene, New J. Chem., 2008, 32(3), 477-482.

28 L. Veltri, V. Maltese, F. Auriemma, C. Santillo, S. Cospito, M. La Deda, G. Chidichimo, B. Gabriele, C. De Rosa and A. Beneduci, Mesophase Tuning in Discotic Dimers $\pi$ Conjugated Ionic Liquid Crystals through Supramolecular Interactions and the Thermal History, Cryst. Growth Des., 2016, 16(10), 5646-5656.

29 A. Beneduci, S. Cospito, A. Crispini, B. Gabriele, F. P. Nicoletta, L. Veltri and G. Chidichimo, Switching from Columnar to Calamitic Mesophases in a New Class of Rod-like Thienoviologens, J. Mater. Chem. C, 2013, 1(11), 2233-2240.

30 W.-L. He, Z. Yang, H. Cao, D. Wang, D.-K. Yang and H. Yang, Chiral Hydrogen-Bonded Complex with Different Mesogens Length and Its Effect on the Performances of Blue Phase, Opt. Mater. Express, 2016, 6(3), 868-875.

31 W. He, G. Pan, Z. Yang, D. Zhao, C. Niu, W. Huang, X. Yuan, J. Cuo, H. Cao and H. Yang, Wide Blue Phase Range in a Hydrogen-Bonded Self-Assembled Complex of Chiral Fluoro-Substituted Benzoic Acid and Pyridine Derivative, Adv. Mater., 2009, 21(20), 2050-2053.

32 Y. Wang, H. Shang, B. Li, H. Zhang and S. Jiang, Modulating the Assembly of: N-Benzylideneaniline by Halogen Bonding: Crystal, Cocrystal and Liquid Crystals, CrystEngComm, 2017, 19(27), 3801-3807.

33 O. S. Bushuyev, A. Tomberg, T. Friščić and C. J. Barrett, Shaping Crystals with Light: Crystal-to-Crystal Isomerization and Photomechanical Effect in Fluorinated Azobenzenes, J. Am. Chem. Soc., 2013, 135(34), 1255612559.

34 R. Weiss and F. G. Pühlhofer, Electrostatics and Color: Massive Electrostatic Perturbation of Chromophores by Ion Cluster Ligands, J. Am. Chem. Soc., 2007, 129(3), 547553.

35 V. Kumar, D. J. Mulder, G. Cavallo, T. Pilati, G. Terraneo, G. Resnati, A. P. H. J. Schenning and P. Metrangolo, Structural Characterization of New Fluorinated Mesogens Obtained through Halogen-Bond Driven Self-Assembly, $J$. Fluorine Chem., 2017, 198(2016), 54-60.

36 H. C. Lin and J. Hendrianto, Synthesis and Characterization of H-Bonded Side-Chain and Crosslinking LC Polymers Containing Donor/acceptor Homopolymers and Copolymers, Polymer, 2005, 46(26), 12146-12157.

37 W. L. He, H. Gu, P. Zhao, Z. Yang, H. Cao and D. Wang, Synthesis and Mesophase Behaviour of Branched Azobenzene-Based Supramolecular Hydrogen-Bonded Liquid Crystals, Liq. Cryst., 2017, 44(3), 593-602.

38 J. T. Carli, C. D. Lindberg, M. D. Heltne, E. C. Bornowski, E. A. John and K. N. Wiegel, Supramolecular Main-Chain Liquid Crystalline Polymers with Competitive Hydrogen Bonding: Inclusion of Structurally Analogous Hydrogen Bond Acceptors and the Effects on Liquid Crystallinity, Mol. Cryst. Liq. Cryst., 2017, 656(1), 83-88.

39 J. R. Greuel, D. K. Witte, C. M. Morales and K. N. Wiegel, Supramolecular Main-Chain Liquid Crystalline Polymers 
and Networks with Competitive Hydrogen Bonding, Liq. Cryst., 2010, 37(9), 1127-1131.

40 H. A. Ahmed, M. Hagar, M. Alaasar and M. Naoum, Wide Nematic Phases Induced by Hydrogen-Bonding, Liq. Cryst., 2018, 46(4), 550-559.

41 M. Alaasar, S. Poppe, Q. Dong, F. Liu and C. Tschierske, Mirror Symmetry Breaking in Cubic Phases and Isotropic Liquids Driven by Hydrogen Bonding, Chem. Commun., 2016, 52(96), 13869-13872.

42 D. Janietz and M. Bauer, Chromophoric poly(viny1 Alcohol Derivative)s, 1 Synthesis and Spectroscopical Characterization of Some poly(viny1 alcoho1)s with Alkoxyazobenzenecarbonyl Substituents, Macromol. Chem. Phys., 1991, 2635-2640.

43 H. A. Ahmed, M. M. Naoum and G. R. Saad, Mesophase Behaviour of 1:1 Mixtures of 4-N-Alkoxyphenylazo Benzoic Acids Bearing Terminal Alkoxy Groups of Different Chain Lengths, Liq. Cryst., 2016, 43(9), 1259-1267.

44 R. Wang, G. Lee and C. K. Lai, Effect of Counter Ions on the Mesogenic Ionic N-Phenylpyridiniums, CrystEngComm, 2018, 20(18), 2593-2607.

45 T. D. Michels, J. U. Rhee and C. D. Vanderwal, Synthesis of $\delta$-Tributylstannyl-A, $\gamma, \delta$-Unsaturated Aldehydes from Pyridines, Org. Lett., 2008, 10(21), 4787-4790.

46 (a) N. Zeghbib, P. Thelliere, M. Rivard and T. Martens, Microwaves and Aqueous Solvents Promote the Reaction of Poorly Nucleophilic Anilines with a Zincke Salt, J. Org. Chem., 2016, 81, 3256-3262; (b) J. Tang, R. Huang, H. Gao, X. Cheng, M. Prehm and C. Tschierske, Columnar Mesophases of Luminescent Polycatenar Liquid Crystals Incorporating a 1,3-Substituted Benzene Ring Interconnecting Two 1,3,4-Oxadiazoles, RSC Adv., 2012, 2842-2847.

47 T. Kuwabara, X. Tao, H. Guo and M. Katsumata, Monocationic Ionophores Capable of Ion-Responsive Intramolecular Charge Transfer Absorption Variation, Tetrahedron, 2016, 72(8), 1069-1075.

48 H. Kuo, W. Ko, Y. Hsu, G. Lee and C. K. Lai, Mesogenic Heterocycles Derived from Quinoxaline Schiff Bases, Tetrahedron, 2016, 72(41), 6321-6333.

49 R. Chen, Q. Weng, Z. An, S. Zhu, Q. Wang and X. Chen, Investigation of 4-Pyridyl Liquid Crystals on the Photovoltaic Performance and Stability of Dye Sensitized Solar Cells by the Co-Sensitization, Dyes Pigm., 2018, 159, 527-532.

50 R. Chen, Y. Qin, Z. An, X. Chen and P. Chen, The Effect of Phenyl Ring on the Physical Properties of Liquid Crystals Containing 4-Pyridyl Terminal Group, Liq. Cryst., 2018, 45(12), 1825-1833.

51 R. Chen, Z. An, W. Wang, X. Chen and P. Chen, Improving UV Stability of Tolane-Liquid Crystals in Photonic Applications by the Ortho Fluorine Substitution, opt. Mater. Express, 2016, 6(1), 1585-1587.

52 Z. Wu, Z. An, X. Chen and P. Chen, Cyclic Thiourea/Urea Functionalized Triphenylamine-Based Dyes for HighPerformance Dye-Sensitized Solar Cells, Org. Lett., 2013, 15(7), 1456-1459.
53 D.-J. Mulder, T. Liang, Y. Xu, J. ter Schiphorst, L. M. W. Scheres, B. M. Oosterlaken, Z. Borneman, K. Nijmeijer and A. P. H. J. Schenning, Proton Conductive Cationic Nanoporous Polymers Based on Smectic Liquid Crystal Hydrogen-Bonded Heterodimers, J. Mater. Chem. C, 2018, 6(18), 5018-5024.

54 B. B. D. Shaw and E. A. Wagstaff, Nitration of $\beta$ Phenylethylpyridines and Relateded Compounds. Part I, J. Chem. Soc., 1933, 77-79.

55 M.-C. Chiang and W. H. Hartung, Synthesis of Some Stilbazole Derivatives, J. Org. Chem., 1945, 10(1), 21-25.

56 J. E. Stumpel, D. Liu, D. J. Broer and A. P. H. J. Schenning, Photoswitchable Hydrogel Surface Topographies by Polymerisation-Induced Diffusion, Chem.-Eur. J., 2013, 19, 10922-10927.

57 M. Saccone, K. Kuntze, Z. Ahmed, S. Antti, M. Gieseb and A. Priimagi, Ortho-Fluorination of Azophenols Increases the Mesophase Stability of Photoresponsive HydrogenBonded Liquid Crystals, J. Mater. Chem. C, 2018, 6(37), 9958-9963.

58 D. W. Bruce, D. A. Dunmur, E. Lalinde, P. M. Maitlis and P. Styring, 4-Alkyloxy-4'-Stilbazoles New Heterocyclic Mesogens, Liq. Cryst., 1988, 3(3), 385-395.

59 R.-T. Wang, G.-H. Lee and C. K. Lai, Anion-Induced Ionic Liquid Crystals of Diphenylviologens, J. Mater. Chem. C, 2018, 6(35), 9430-9444.

60 M. Nanasawa, M. Miwa and M. Hirai, Synthesis of Viologens with Extended $\pi$-Conjugation and Their Photochromic Behavior on Near-IR Absorption, J. Org. Chem., 2000, 593-595.

61 S. G. Bhat, G. S. Ramachandra, P. Bhagavath, M. Subrao, D. M. Potukuchi and S. Maddasani, Self-Assembled Liquid Crystalline Materials with Fatty Acids, J. Therm. Anal. Calorim., 2018, 132(2), 989-1000.

62 S. G. Bhat, M. Srinivasulu, S. R. Girish, Padmalatha, P. Bhagavath, S. Mahabaleshwara, D. M. Potukuchi and M. Muniprasad, Influence of Moieties and Chain Length on the Abundance of Orthogonal and Tilted Phases of Linear Hydrogen-Bonded Liquid Crystals, Py16BA: nOBAs, Mol. Cryst. Liq. Cryst., 2012, 552(1), 83-96.

63 L. Ong, S. Ha, G. Yeap and H. Lin, Heterocyclic PyridineBased Liquid Crystals: Synthesis and Mesomorphic Properties, Liq. Cryst., 2018, 45(11), 1574-1584.

64 S. Ha, L. Ong, Y. Win, Y. Sivasothy and B. Barat, The Synthesis and Characterization of New Schiff Bases: 4[(Pyridin-4-Ylmethylene)Amino]Phenylalkanoates, Aust. J. Basic Appl. Sci., 2010, 4(6), 1146-1151.

65 O. N. Kadkin, H. Han and Y. G. Galyametdinov, Synthesis, Computational Modelling and Liquid Crystalline Properties of Some [3] Ferrocenophane-Containing Schiff'S Bases and B-Aminovinylketone: Molecular Geometry - Phase Behaviour Relationship, J. Organomet. Chem., 2007, 692, 5571-5582.

66 Y. C. Lim, S. Ha, G. Yeap and S. S. Sastry, Synthesis and Mesomorphic Properties of New Heterocyclic Liquid Crystals with Ester-Chalcone Central Linkages, J. Taibah Univ. Sci., 2017, 11(1), 133-140. 
67 Y. Sakurai, S. Takenaka and H. Morita, Molecular Structure and Smectic Properties. Part 1. The Effect of Linkages on Smectic A Thermal Stability in Three Aromatic Ring Compounds Linked by Ester Groups, J. Chem. Soc., Perkin Trans. 2, 1989, 1199-1204.

68 M. Du, L. Li, J. Zhang, K. Li, M. Cao, L. Mo, G. Hu, Y. Chen, $\mathrm{H}$. Yu and $\mathrm{H}$. Yang, Photoresponsive Iodine-Bonded Liquid Crystals Based on Azopyridine Derivatives with a Low Phase-Transition Temperature, Liq. Cryst., 2019, 46(1), 3744.

69 L. González, N. Gimeno, R. M. Tejedor, V. Polo, M. B. Ros, S. Uriel and osé L. Serrano, Halogen-Bonding Complexes Based on Bis(iodoethynyl)benzene units: A New Versatile Route to Supramolecular Materials, Chem. Mater., 2013, 25(22), 1711-1713.

70 M. Alaasar and C. Tschierske, Nematic Phases Driven by Hydrogen-Bonding in Liquid Crystalline Nonsymmetric Dimers, Liq. Cryst., 2019, 46(1), 124-130.

71 M. M. Naoum, A. A. Fahmi and M. A. Alaasar, Supramolecular Hydrogen-Bonded Liquid Crystals Formed from 4-(4'-Pyridylazophenyl)-4"'-alkoxy Benzoates and 4-Substituted Benzoic Acids, Mol. Cryst. Liq. Cryst., 2008, 487(1), 74-91.

72 M. Alaasar and C. Tschierske, Non-Symmetric Ether-Linked Liquid Crystalline Dimers with a Highly Polar End Group, Liq. Cryst., 2016, 44(2), 387-393.

73 S. M. Jansze, A. Martínez-felipe, J. M. D. Storey, A. T. M. Marcelis and C. T. Imrie, A Twist-Bend Nematic Phase Driven by Hydrogen Bonding **, Angew. Chem., 2015, 127, 653-656.

74 R. Chen, L. Zhao, Z. An, X. Chen and P. Chen, Synthesis and Properties of Allyloxy-Based Tolane Liquid Crystals with High Negative Dielectric Anisotropy, Liq. Cryst., 2017, 44(14-15), 2184-2191.

75 R. Walker, D. Pociecha, J. P. Abberley, A. Martinez-Felipe, D. A. Paterson, E. Forsyth, G. B. Lawrence, P. A. Henderson, J. M. D. Storey, E. Gorecka, et al., Spontaneous Chirality through Mixing Achiral Components: A Twist-Bend Nematic Phase Driven by Hydrogen-Bonding between Unlike Components, Chem. Commun., 2018, 54(27), 3383-3386.

76 J. P. Abberley, S. M. Jansze, R. Walker, D. A. Paterson, P. A. Henderson, A. T. M. Marcelis, J. M. D. Storey, T. Corrie, J. P. Abberley, S. M. Jansze, et al., StructureProperty Relationships in Twist-Bend Nematogens: The Influence of Terminal Groups, Liq. Cryst., 2017, 44(1), 6883.

77 B. B. Chakraborty, S. Anwar, S. Das, S. B. Paul, J. De and S. Choudhury, Aggregation Dependent Fluorescence Switching in Benzothiazole Derivative Based H-Bonded Mesogen, Liq. Cryst., 2018, 45(11), 1644-1653.

78 B. Maleki and H. Salehabadi, Ammonium Chloride; as a Mild and Efficient Catalyst for the Synthesis of Some 2-arylbenzothiazoles and Bisbenzothiazole Derivatives, Eur. J. Chem., 2010, 1(4), 377-380.

79 F. S. Spring and T. Vickersta, The Behaviour of Keten in the Friedel-Crafts Reaction, J. Am. Chem. Soc., 1925, 47, 2777.
80 K. Goossens, K. Lava, C. W. Bielawski and K. Binnemans, Ionic Liquid Crystals: Versatile Materials, Chem. Rev., 2016, 116(8), 4643-4807.

81 P. K. Bhowmik, S. T. Killarney, J. R. A. Li, J. J. Koh, H. Han, L. Sharpnack, D. M. Agra-kooijman, M. R. Fisch and S. Kumar, Thermotropic Liquid-Crystalline Properties of Extended Viologen Bis(Triflimide) Salts, Liq. Cryst., 2018, 45(6), 872-885.

82 P. K. Bhowmik, A. Chang, J. Kim, E. J. Dizon, R. C. G. Principe and H. Han, Thermotropic LiquidCrystalline Properties of Viologens Containing 4- $\mathrm{N}$ Alkylbenzenesulfonates $\dagger$, Crystals, 2019, 9(2), 77.

83 G. Casella, V. Causin, F. Rastrelli and G. Saielli, Ionic Liquid Crystals Based on Viologen Dimers: Tuning the Mesomorphism by Varying the Conformational Freedom of the Ionic Layer, Liq. Cryst., 2016, 43(9), 1161-1173.

84 I. Pibiri, A. Beneduci, M. Carraro, V. Causin, G. Casella, G. Chidichimo, G. A. Corrente, A. Pace, A. Riccobono and G. Saiellie, Mesomorphic and Electrooptical Properties of Viologens Based on Non-Symmetric Alkyl/polyfluoroalkyl Functionalization and on Oxadiazolyl-Extended Bent Core, J. Mater. Chem. C, 2019, 7(26), 7974-7983.

85 T. N. Ahipa and A. V. Adhikari, 2-Methoxypyridine Derivatives: Synthesis, Liquid Crystalline and PhotoPhysical Properties, New J. Chem., 2014, 38(10), 5018-5029.

86 T. N. Ahipa and A. V. Adhikari, Trihydrazone Functionalized Cyanopyridine Discoids: Synthesis, Mesogenic and Optical Properties, Tetrahedron Lett., 2014, 55(2), 495-500.

87 T. N. Ahipa, V. Kumar, D. S. Shankar Rao, S. K. Prasad and A. V. Adhikari, New 4-(2-(4-Alkoxyphenyl)-6Methoxypyridin-4-Yl)benzonitriles: Synthesis, Liquid Crystalline Behavior and Photo Physical Properties, CrystEngComm, 2014, 16(25), 5573-5582.

88 S. J. Lee, J. Y. Jho and J. H. Lee, Supramolecular Discotic Columnar Liquid Crystals Built through Single Hydrogen Bonding between Carboxylic Acid and Pyridine Moieties, Mol. Cryst. Liq. Cryst., 2015, 621(1), 169-174.

89 S. J. Lee, M. K. You, S. W. Lee, J. Lee, J. H. Lee and J. Y. Jho, Star-Shaped Supramolecular Liquid Crystals Formed by Hydrogen Bonding between Phloroglucinol and Stilbazole Derivatives with Different Molecular Shapes, Liq. Cryst., 2011, 38(10), 1289-1299.

90 R. L. Coelho, E. Westphal, D. Z. Mezalira and H. Gallardo, Polycatenar Liquid Crystals Based on Bent-Shaped Chalcone and Cyanopyridine Molecules, Liq. Cryst., 2017, 44(2), 405-416.

91 A. G. Dal Bó, Y. M. S. Micheletto, F. C. Giacomelli, G. Lopez, M. J. R. Sartor, J. Rafique, S. Saba, L. D. M. D. Silveira, J. Mendes and T. E. A. Frizon, Synthesis of New Monodendrons, Gallic Acid Derivatives, Self- Assembled in a Columnar Phase, Liq. Cryst., 2016, 43(3), 292-304.

92 A. S. Achalkumar, D. S. Shankar Rao and C. V. Yelamaggad, Non-Symmetric Dimers Comprising Chalcone and Cholesterol Entities: An Investigation on StructureProperty Correlations, New J. Chem., 2014, 38(9), 4235-4248. 
93 T. N. Ahipa, V. Kumar and A. V. Adhikari, New Columnar Liquid Crystal Materials Based on Luminescent 2Methoxy-3-Cyanopyridines, Struct. Chem., 2014, 25(4), 1165-1174.

94 J. H. Lee, Supramolecular Discotic Nematic Liquid Crystals Built through Simple Hydrogen Bonding between 1,3,5tris(4-Hydroxyphenyl)benzene and Stilbazole Derivatives, Mol. Cryst. Liq. Cryst., 2016, 635(1), 133-138.

95 M. Pfletscher, C. Wölper, J. S. Gutmann, M. Mezger and M. Giese, A Modular Approach towards Functional Supramolecular Aggregates-Subtle Structural Differences Inducing Liquid Crystallinity, Chem. Commun., 2016, 52(55), 8549-8552.

96 M. Spengler, R. Y. Dong, C. A. Michal, M. Pfletscher and M. Giese, Fluorination of Supramolecular Liquid CrystalsTuning Tool and Analytical Probe, J. Mater. Chem. C, 2017, 5(9), 2235-2239.

97 D. R. Vinayakumar, H. Ulla, S. Kumar, A. Pandith, M. N. Satyanarayan, D. S. S. Rao, S. K. Prasad and A. V. Adhikari, Hydrogen Bond-Driven Columnar SelfAssembly of Electroluminescent D-A-D Configured Cyanopyridones, J. Mater. Chem. C, 2018, 6(27), 7385-7399.

98 X. Wang, L. Bai, S. Kong, Y. Song and F. Meng, Star-Shaped Supramolecular Ionic Liquid Crystals Based on Pyridinium Salts, Liq. Cryst., 2019, 46(4), 512-522.

99 H. A. Ahmed and M. M. Naoum, Mesophase Behaviour of Azobenzene-Based Angular Supramolecular HydrogenBonded Liquid Crystals, Liq. Cryst., 2016, 43(2), 222-234.

100 M. M. Naoum, A. A. Fahmi, A. A. Refaie and M. A. Alaasar, Novel Hydrogen-Bonded Angular Supramolecular Liquid Crystals, Liq. Cryst., 2012, 39(1), 47-61.

101 M. Lizu, M. R. Lutfor, N. L. Surugau, S. E. How and S. E. Arshad, Synthesis and Characterization of Ethyl Cellulose-Based Liquid Crystals Containing Azobenzene Chromophores, Mol. Cryst. Liq. Cryst., 2010, 528, 64-73.

102 C. C. Han, Y. C. Chou, S. Y. Chen and H. C. Lin, HydrogenBonded Bent-Core Blue Phase Liquid Crystal Complexes Containing Various Molar Ratios of Proton Acceptors and Donors, RSC Adv., 2016, 6(38), 32319-32327.

103 C. C. Han, L. H. Yang, P. Raghunath, M. C. Lin, R. Kumar and H. C. Lin, Lateral Fluoro-Substitution and Chiral Effects on Supramolecular Liquid Crystals Containing Rod-like and H-Bonded Bent-Core Mesogens, RSC Adv., 2016, 6(112), 110482-110492.

104 N. Trišović, J. Antanasijević, J. Rogan, D. Poleti, T. TóthKatona, M. Salamonczyk, A. Jákli and K. Fodor-Csorba, Investigation of Supramolecular Architectures of BentShaped Pyridine Derivatives: From a Three-Ring Crystalline Compound towards Five-Ring Mesogens, New J. Chem., 2016, 40(8), 6977-6985.

105 E. D. Bergmann and S. Pinchas, Condensation Reactions of 2,6-Lutidine and an Estrogenic 2,6-Distyrylpyridine Derivative, J. Org. Chem., 1950, 15(6), 1184-1190.

106 S. Sivakumar, M. L. P. Reddy, A. H. Cowley and K. V. Vasudevan, Synthesis and Crystal Structures of Lanthanide 4-Benzyloxy Benzoates: Influence of ElectronWithdrawing and Electron-Donating Groups on
Luminescent Properties, Dalton Trans., 2010, 39(3), 776786.

107 J. M. Marković, N. P. Trišović, T. Tóth-Katona, M. K. Milčić, A. D. Marinković, C. Zhang, A. J. Jákli and K. Fodor-Csorba, A Structure-Property Relationship Study of Bent-Core Mesogens with Pyridine as the Central Unit, New J. Chem., 2014, 38(4), 1751-1760.

108 B. Pradhan, R. K. Gupta, S. K. Pathak, J. De, S. K. Pal and A. S. Achalkumar, Columnar Self-Assembly of Luminescent Bent-Shaped Hexacatenars with a Central Pyridine Core Connected with Substituted 1,3,4Oxadiazole and Thiadiazoles, New J. Chem., 2018, 42(5), 3781-3798.

109 B. Pradhan, S. K. Pathak, R. K. Gupta, M. Gupta, S. K. Pal and A. S. Achalkumara, Star-Shaped Fluorescent Liquid Crystals Derived from S-Triazine and 1,3,4-Oxadiazole Moieties, J. Mater. Chem. C, 2016, 4(25), 6117-6130.

110 S. K. Pathak, S. Nath, R. K. Gupta, D. S. S. Rao, S. K. Prasad and A. S. Achalkumar, Effect of Regioisomerism on the SelfAssembly and Photophysical Behavior of 1,3,4-Thiadiazole Based Polycatenars, J. Mater. Chem. C, 2015, 3(31), 81668182.

111 M. Pfletscher, S. Hölscher, C. Wölper, M. Mezger and M. Giese, Structure-Property Relationships in HydrogenBonded Liquid Crystals, Chem. Mater., 2017, 29(19), 84628471.

112 A. Pană, M. Iliș, T. Staicu, I. Pasuk and V. Cîrcu, Columnar Bis(Pyridinium) Ionic Liquid Crystals Derived from 4Hydroxypyridine: Synthesis, Mesomorphism and Emission Properties, Liq. Cryst., 2016, 43(3), 381-392.

113 S. Chen, H. Yuan, S. Chen, H. Yang, Z. Ge, H. Zhuo and J. Liu, Development of Supramolecular Liquid-Crystalline Polyurethane Complexes Exhibiting Triple-Shape Functionality Using a One-Step Programming Process, $J$. Mater. Chem. A, 2014, 2(26), 10169-10181.

114 S. Chen, F. Mo, S. Chen, Z. Ge, H. Yang, J. Zuo, X. Liu and H. Zhuo, New Insights into Multi-Shape Memory Behaviours and Liquid Crystalline Properties of Supramolecular Polyurethane Complexes Based on Pyridine-Containing Polyurethane and 4Octyldecyloxybenzoic Acid, J. Mater. Chem. A, 2015, 3(38), 19525-19538.

115 A. K. A. Al-Lami, Synthesis and Characterization of Hydrogen Bonded Liquid Crystalline Block Copolymers Based on a Schiff Base Moiety, J. Polym. Res., 2015, 22(1), 625.

116 F. Ignatious, R. W. Lenz and S. W. Kantor, Synthesis and Characterization of Block Copolymers Containing Rigid Liquid Crystalline and Flexible Butylene Terephthalate Segments, Macromolecules, 1994, 27, 5248-5257.

117 J. Wang, S. Ma, X. Chen and H. Chen, Hydrogen-Bonded Liquid Crystalline Polymers Containing poly(4vinylpridine) and Dendron-like Side Chains: From lamellar to Columnar Phase, Mater. Today Commun., 2015, 4, 77-85.

118 S. Q. Yang, W. Qu, H. B. Pan, Y. D. Zhang, S. J. Zheng, X. H. Fan and Z. Shen, Effects of Main Chain and 
Acceptor Content on Phase Behaviors of Hydrogen-Bonded Main-Chain/side-Chain Combined Liquid Crystalline Polymers, Polymer, 2016, 84, 355-364.

119 D. Zhang, Y. X. Liu, X. H. Wan and Q. F. Zhou, Synthesis and Characterization of a New Series Of "mesogenJacketed Liquid Crystal Polymers"based on the Newly Synthesized Vinylterephthalic Acid, Macromolecules, 1999, 32(16), 5183-5185.

120 W. Qu, X. Zhu, J. Chen, L. Niu, D. Liang, X. Fan, Z. Shen and Q. Zhou, Synthesis and Characterization of a MesogenJacketed Polyelectrolyte, Macromolecules, 2014, 47(8), 2727-2735.

121 C. Xing, J. W. Y. Lam, K. Zhao and B. Z. Tang, Synthesis and Liquid Crystalline Properties of Poly(1-Alkyne)s Carrying Triphenylene Discogens, J. Polym. Sci., Part A: Polym. Chem., 2008, 46(9), 2960-2974.

122 Y. Zhu, M. Zheng, Y. Tu and X. Chen, Supramolecular Fluorescent Polymers Containing $\alpha$-Cyanostilbene-Based Stereoisomers: $Z / E$-Isomerization Induced Multiple Reversible Switching, Macromolecules, 2018, 51(9), 34873496.

123 M. Goh, S. Matsushita and K. Akagi, From Helical Polyacetylene to Helical Graphite: Synthesis in the Chiral Nematic Liquid Crystal Field and Morphology-Retaining Carbonisation, Chem. Soc. Rev., 2010, 39(7), 2466-2476.

124 D. Z. L. L. M. Lu, Dimeric Liquid Crystalline Thermosets from Azo-Containing Diglycidyl Ether Cured by Anhydride, Polym. Bull., 2011, 66(8), 1111-1123.

125 S. Sundaram, P. Subhasri, T. R. Rajasekaran, R. Jayaprakasam and T. S. Senthil, Induced Smectic X
Phase Through Intermolecular Hydrogen-Bonded Liquid Crystals Formed Between Citric Acid and P-N-(Octyloxy) Benzoic Acid, Braz. J. Phys., 2017, 47(4), 382-392.

126 S. Leng, H. Chan, J. Jing, J. Hu, R. M. Moustafa, R. M. Van Horn, M. J. Graham, B. Sun, M. Zhu, K. Jeong, et al., From Crystals to Columnar Liquid Crystal Phases: Molecular Design, Synthesis and Phase Structure Characterization of a Series of Novel Phenazines Potentially Useful in Photovoltaic Applications, Soft Matter, 2010, 6(1), 100-112.

127 M. Lehmann, M. Jahr, B. Donnio, R. Graf, S. Gemming and I. Popov, Star-Shaped Oligobenzoates: Non-Conventional Mesogens Forming, Chem.-Eur. J., 2008, 14(12), 3562-3576.

128 C. Luo, Y. Jia, B. Sun and F. Meng, Effect of Chain Length in the Terminal Group on Mesomorphic Behavior of Novel (-)-Menthol-Based Chiral Liquid Crystal Compounds with Blue Phase, New J. Chem., 2017, 41(9), 3677-3686.

129 J. Kirchhoff, L. S. Hirst, K. M. Fergusson and M. Hird, Low Electric-Field-Induced Switching in the B 1 Bent-Core Liquid Crystal Phase, Appl. Phys. Lett., 2007, 90(16), 161905.

130 G. Shanker and C. V. Yelamaggad, Synthesis and Thermal Behavior of Chiral Dimers: Occurrence of Highly Frustrated and Cholesteric Liquid Crystal Phases, New J. Chem., 2012, 36(4), 918-926.

131 M. G. Tamba, S. M. Salili, C. Zhang, A. Jakli, G. H. Mehl, R. Stannarius and A. Eremin, A Fibre Forming Smectic Twist-bent Liquid Crystalline Phase, RSC Adv., 2015, 5(15), 11207-11211. 